\title{
Low Frequency Waves Upstream and Downstream of the Terrestrial Bow Shock
}

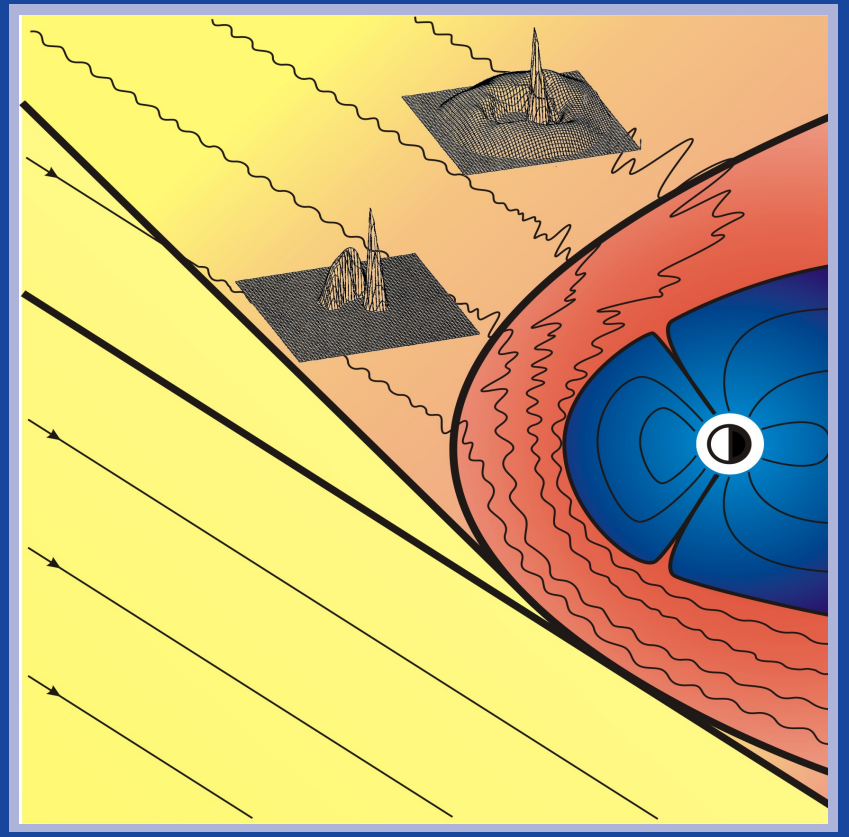

\section{Yasuhito Narita}

International Max Planck Research School on Physical Processes in the Solar System and Beyond at the Universities of Braunschweig and Göttingen 


\section{Low Frequency Waves Upstream and Downstream of the Terrestrial Bow Shock}

Von der Fakultät für Physik und Geowissenschaften der Technischen Universität Carolo-Wilhelmina zu Braunschweig zur Erlangung des Grades eines Doktors der Naturwissenschaften

(Dr.rer.nat.) genehmigte Dissertation von Yasuhito Narita aus Nagoya, Aichi, Japan 


\section{Bibliografische Information Der Deutschen Bibliothek}

Die Deutsche Bibliothek verzeichnet diese Publikation in der Deutschen Nationalbibliografie; detaillierte bibliografische Daten sind im Internet über http: //dnb. ddb. de abrufbar.

1. Referentin oder Referent: Prof. Dr. Karl-Heinz Glaßmeier

2. Referentin oder Referent: Prof. Dr. Uwe Motschmann

3. Referentin oder Referent: Prof. Dr. Toshifumi Mukai eingereicht am: 26. Oktober 2005

mündliche Prüfung (Disputation) am: 16. Februar 2006

Copyright (c) Copernicus GmbH 2006

ISBN 3-936586-50-0

Copernicus GmbH, Katlenburg-Lindau

Druck: Schaltungsdienst Lange, Berlin

Printed in Germany 


\section{$\underline{\text { Vorabveröffentlichungen der Dissertation }}$}

Teilergebnisse aus dieser Arbeit wurden mit Genehmigung der Fakultät für Physik und Geowissenschaften, vertreten durch den Mentor der Arbeit, in folgenden Beiträgen vorab veröffentlicht:

Publikationen:

Narita, Y., K.-H. Glassmeier, S. Schäfer, U. Motschmann, K. Sauer, I. Dandouras, K.H. Fornaçon, E. Georgescu und H. Rème, Dispersion analysis of ULF waves in the foreshock using cluster data and the wave telescope technique, Geophys. Res. Lett, 30, SSC 43-1, CiteID 1710, doi:10.1029/2003GL017432, 2003.

Narita, Y., K.-H. Glassmeier, S. Schäfer, U. Motschmann, M. Fränz, I. Dandouras, K.H. Fornaçon, E. Georgescu, A. Korth, H. Rème und I. Richter, Alfvén waves in the foreshock propagating upstream in the plasma rest frame: statistics from Cluster observations, Ann. Geophysicae, 22, 2315-2323, 2004.

Gurgiolo, C., M. L. Goldstein, Y. Narita, K.-H. Glassmeier und A. N. Fazakerley, A phase locking mechanism for non-gyrotropic electron distributions upstream of the Earth's bow shock, J. Geophys. Res., 110, A06206, doi:10.1029/2005JA011010, 2005.

Eastwood, J. P., E. A. Lucek, C. Mazelle, K. Meziane, Y. Narita, J. Pickett und R. A. Treumann, The foreshock, Space Sci. Rev., 118, 41-94, doi:10.1007/s11214-005-38243, 2005 .

Narita, Y. und K.-H. Glassmeier Dispersion analysis of low-frequency waves through the terrestrial bow shock, J. Geophys. Res., 110, A12215, doi:10.1029/2005JA011256, 2005 .

Narita, Y. und K.-H. Glassmeier, Low-frequency waves in the bow shock environment, Proc. of the Cluster and Double Star Symposium - 5th Anniversary of Cluster in Space, (Ed) K. Fletcher, SP-598, ISBN 92-9092-909-X, ISSN 1609-042X, ESA Publications Division, The Netherlands, January 2006.

Narita, Y., K.-H. Glassmeier, K.-H. Fornaçon, I. Richter, S. Schäfer, U. Motschmann, I. Dandouras, H. Rème und E. Georgescu, Low frequency wave characteristics in the upstream and downstream regime of the terrestrial bow shock, J. Geophys. Res., 111, A01203, doi:10.1029/2005JA011231, 2006. 
Narita, Y., K.-H. Glassmeier und R. A. Treumann, Magnetic turbulence spectra in the high-beta plasma upstream of the terrestrial bow shock Phys. Rev. Lett., eingereicht, 2006.

Narita, Y., Low frequency waves upstream and downstream of the terrestrial bow shock, Planet. Space Sci., eingereicht, 2006.

Tagungsbeiträge:

AGU (Americal Geophysical Union) Fall Meeting 2002, San Francisco, Vereinigten Staaten von Amerika, 9. Dez. 2002 (Polarization and dispersion analysis of ULF waves using Cluster data, poster)

ISSI (International Space Science Institute) 1st Workshop on dayside magnetospheric boundaries, Bern, Schweiz, 19. Mär. 2003 (ULF wave analysis in the magnetosheath and upstream solar wind - short report from Braunschweig/FGM team, talk)

EGS-AGU-EUG Joint Assembly, Nizza, Frankreich, 7. Apr. 2003, (Wave propagating directions in the terrestrial magnetosheath, poster)

EGS-AGU-EUG Joint Assembly, Nizza, Frankreich, 8. Apr. 2003, (Dispersion Analysis and Wave Mode Identification of Magnetosheath Fluctuations: Is the MHD Assumption Justified? - A Case Study, talk)

STAMMS (Spatio-Temporal Analysis and Multi-point Measurements in Space), Orléans, Frankreich, 13. Mai 2003 (Application of wave telescope - foreshock wave study, poster)

IUGG (International Union of Geophysics and Geodesy) General Assembly 2003, Sapporo, Japan, 30. Jun. 2003 (Dispersion analysis of ULF waves in the foreshock using Cluster data, talk)

ISSI 2nd Workshop on dayside magnetospheric boundaries, Bern, Schweiz, 12. Nov. 2003 (Wave modes and large scale wave distribution -1. foreshock, - 2. magnetosheath, talks)

EGU (European Geosciences Union) General Assembly, Nizza, Frankreich, 30. Apr. 2004 (Dispersion relation and wave propagation in the foreshock and magnetosheath: Low-frequency picture from Cluster, poster)

Cluster FGM Workshop, London, Großbritanien, Mär. 2005, (Low frequency waves upstream and downstream of the bow shock, talk)

Magnetometer workshop, Ibenhorst, Born, Deutschland, 12. Apr. 2005, (Low frequency waves upstream and downstream of the bow shock, talk)

EGU General Assembly, Wien, Österreich, 29. Apr. 2005, (A statistical picture of low 
frequency waves from the upstream to the downstream region of the terrestrial bow shock - Cluster observations, talk)

IAGA (International Association of Geomagnetism and Aeronomy) Scientific Assembly, Toulouse, Frankreich, 20. Jul. 2005 (Evidence of low frequency turbulence in the near-Earth solar wind plasma, talk)

STIMGM Workshop (Solar - Terrestrial Interactions from Microscale to Global Models), Sinaia, Rumänien, 9. Sep. 2005 (Magnetic turbulence, talk; Low-frequency waves in the magnetosphere - a view from multi-point measurements, invited talk)

Cluster and Double Star symposium - 5th anniversary of Cluster in Space, ESTEC, Noordwijk, Niederlande, 21. Sep. 2005, (Low frequency waves in the bow shock environment, poster)

SGEPSS (Society of Geomagnetism and Earth, Planetary and Space Sciences, Japan) annual meeting, Kioto, Japan, 29. und 30. Sep. 2005, (Low frequency waves in the bow shock environment, talk; Magnetic turbulence spectra, poster)

Evaluation Meeting of the International Max Planck Research School, Katlenburg-Lindau, 11. Nov. 2005, (Bow shock upstream and downstream waves, talk) 



\section{Contents}

Vorabveröffentlichungen der Dissertation 3

$\begin{array}{ll}\text { Summary } & 17\end{array}$

1 Introduction $\quad 19$

1.1 Space plasma . . . . . . . . . . . . . . . . . . . . . 19

1.2 Waves and instabilities ................... 20

1.3 Shock waves . . . . . . . . . . . . . . . . . . 20

1.4 Upstream and downstream waves . . . . . . . . . . . . . . . . . . 21

2 Shocks, upstream and downstream waves, turbulence 23

2.1 Collisionless shocks . . . . . . . . . . . . . . . 23

2.1.1 Rankine-Hugoniot relations . . . . . . . . . . . . . . . . . . . . . . . . . . 24

2.1.2 Different types of shocks . . . . . . . . . . . . . 26

2.1 .3 Bow shock . . . . . . . . . . . . . . . . 28

2.2 Upstream waves . . . . . . . . . . . . . . . . . . . . . 31

2.2.1 Ion beam instabilities . . . . . . . . . . . . . . . . 32

2.2.2 Shock foot waves . . . . . . . . . . . . . . . 33

2.3 Downstream waves . . . . . . . . . . . . . . . . . . . . 34

2.3.1 Ion cyclotron instability . . . . . . . . . . . . . . . . . . . . . . . . . . 34

2.3.2 Mirror instability . . . . . . . . . . . . . . . 35

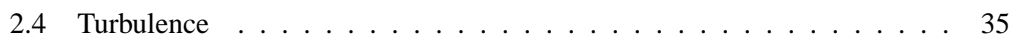

2.4.1 Hydrodynamic turbulence . . . . . . . . . . . . . . 36

2.4.2 Magnetohydrodynamic turbulence ............. 38

2.4 .3 Intermittency . . . . . . . . . . . . . . . . 40

2.4 Turbulence in space . . . . . . . . . . . . . . 40

$3 \quad$ In-situ wave observations $\quad \mathbf{4 3}$

3.1 The Cluster mission . . . . . . . . . . . . . . . . . . . . . . 43

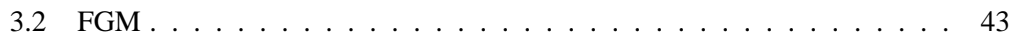

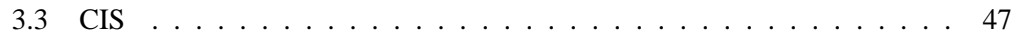

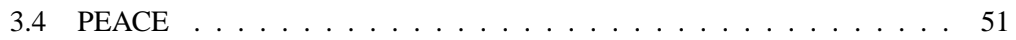

3.5 Wave analyses ........................ 51 
3.5.1 Frequency and wave power . . . . . . . . . . . . 53

3.5.2 Ellipticity . . . . . . . . . . . . . . . . 53

3.5.3 Minimum variance analysis . . . . . . . . . . . . . . . . . 54

3.5.4 Correlation and coherence . . . . . . . . . . . . . . . 54

3.5.5 Wave vector . . . . . . . . . . . . . . . . 55

3.5.6 Frequency and phase velocity in the plasma rest frame . . . . . . 57

3.5.7 Dispersion relation and propagation pattern . . . . . . . . . . 57

4 Dispersion analysis of upstream waves

4.1 Introduction . . . . . . . . . . . . . . . . . . . . . . . . . . . 59

4.2 Dispersion analysis . . . . . . . . . . . . . . . 59

4.3 Dispersion in a beam plasma . . . . . . . . . . . . 63

4.4 Discussion . . . . . . . . . . . . . . . . . . 64

4.5 Summary ............................ 65

5 Wave-particle interaction in the upstream region $\quad 67$

5.1 Introduction . . . . . . . . . . . . . . . . . . 67

5.2 Wave analysis . . . . . . . . . . . . . . . . . 67

5.3 Phase-bunched electrons . . . . . . . . . . . . . . . 70

5.4 Discussion . . . . . . . . . . . . . . . . . . . . . . . . . 74

5.5 Summary . . . . . . . . . . . . . . . . 75

$6 \quad$ Statistical study of upstream waves $\quad 77$

6.1 Introduction . . . . . . . . . . . . . . . . . . . . . 77

6.2 Case study . . . . . . . . . . . . . . . . . . . 77

6.3 Statistical study . . . . . . . . . . . . . . . . . 82

6.4 Discussion . . . . . . . . . . . . . . . . . . . 86

6.5 Summary . . . . . . . . . . . . . . . . . . 87

7 Dispersion analysis of downstream waves $\quad 91$

7.1 Introduction . . . . . . . . . . . . . . . . . . . . . 91

7.2 Dispersion analysis . . . . . . . . . . . . . . . . . . . . . . . . . . .

7.3 Discussion . . . . . . . . . . . . . . . . . 96

7.4 Summary . . . . . . . . . . . . . . . . . . . 99

8 Statistical study of downstream waves $\quad 101$

8.1 Introduction . . . . . . . . . . . . . . . . . . . . . . 101

8.2 Statistical study . . . . . . . . . . . . . . . . . . . . 101

8.3 Results . . . . . . . . . . . . . . . . . . . . . . . . . . 108

8.4 Discussion . . . . . . . . . . . . . . . . . . . 116

8.5 Summary ........................ 121 
9 Magnetic turbulence 131

9.1 Introduction . . . . . . . . . . . . . . . . . . . . 131

9.2 Dispersion curves and $k$-spectra . . . . . . . . . . . . . . . 132

9.3 Discussion . . . . . . . . . . . . . . . . . . . . . . . . . . . 137

9.4 Summary . . . . . . . . . . . . . . . . . . . . . 139

10 Summary and outlook $\quad 141$

$\begin{array}{lr}\text { Acknowledgments } & 159\end{array}$

$\begin{array}{ll}\text { Curriculum Vitae } & 163\end{array}$ 



\section{List of Figures}

2.1 Fast and slow shock wave . . . . . . . . . . . . . . . 25

2.2 Quasi-parallel and quasi-perpendicular shock . . . . . . . . . . . . 26

2.3 Magnetic field profiles across shocks . . . . . . . . . . . . . . . . 28

2.4 Earth's bow shock and magnetosphere . . . . . . . . . . . . . . . . 29

2.5 Hubble Space Telescope image of a bow shock . . . . . . . . . . . . 30

2.6 Image of turbulent water jet . . . . . . . . . . . . . . . . . . . . . . . . 36

2.7 Kolmogorov energy spectrum . . . . . . . . . . . . . . . . 38

2.8 Images of examples of interstellar turbulence . . . . . . . . . . . . . . 39

3.1 Artist's impression of the Cluster spacecraft . . . . . . . . . . . . . . . . 44

3.2 Image of the Cluster spacecraft . . . . . . . . . . . . . . . . 45

3.3 Basic components of a fluxgate magnetometer . . . . . . . . . . . . . . 46

3.4 Image of Cluster FGM . . . . . . . . . . . . . . . . . . . . . . . . . . . . . . . 47

3.5 Image of CODIF and HIA sensor . . . . . . . . . . . . . . . . . . . . . . . 48

3.6 Basic components of HIA . . . . . . . . . . . . . . . . . . . . . . 49

3.7 Basic components of CODIF . . . . . . . . . . . 50

3.8 Basic components of PEACE . . . . . . . . . . . . . . . 51

3.9 Image of PEACE LEEA . . . . . . . . . . . . . . . . . . 52

3.10 Image of PEACE HEEA $\ldots \ldots \ldots \ldots$. . . . . . . . . . . . . . . . . . . . . 52

3.11 Wave power distributions in $k$-domain $\ldots \ldots \ldots . \ldots . \ldots 57$

4.1 Magnetic field and plasma profile in the foreshock . . . . . . . . . . . . . 60

4.2 Dispersion relations in three components . . . . . . . . . . . . . . 61

4.3 Comparison of dispersion relations . . . . . . . . . . . . . . 62

4.4 Ion velocity distribution . . . . . . . . . . . . . . . . . 64

5.1 Magnetic field oscillation upstream of the bow shock . . . . . . . . . 68

5.2 Dynamic wave power spectrograms . . . . . . . . . . . . . . . 69

5.3 Wave power in frequency and wave number domain . . . . . . . . . . 70

5.4 Hodograms of magnetic field oscillation . . . . . . . . . . . . . 71

5.5 Phase-elevation plots showing nongyrotropic electrons . . . . . . . . 71

5.6 PEACE measurement matrix . . . . . . . . . . . . . . . 73

5.7 Phase angle evolution of nongyrotropic electons . . . . . . . . . . . . 74 
6.1 Magnetic field and plasma profile across the bow shock . . . . . . . . . 78

6.2 Configuration of foreshock wave observation . . . . . . . . . . . . 80

6.3 Power spectrum in frequency domain . . . . . . . . . . . . . . . . . . . . . . . 81

6.4 Wave power in $k$-space . . . . . . . . . . . . . . . . 82

6.5 Histogram of wave number . . . . . . . . . . . . . . . . 83

6.6 Spatial distribution of wave phase velocities . . . . . . . . . . . . . . 84

6.7 Foreshock wave properties . . . . . . . . . . . . . . . 86

7.1 Magnetic field and plasma profile across the bow shock . . . . . . . . . . 92

7.2 Cluster orbit . . . . . . . . . . . . . . . . . . . . . . 93

7.3 Dispersion relations and propagation angles in the upstream and the downstream region. . . . . . . . . . . . . . . . . . . 95

7.4 Histograms of magnetic field polarization . . . . . . . . . . . . . . . . . . 96

7.5 Schematic illustration of wave habitats . . . . . . . . . . . . . . . 98

8.1 Magnetic field and plasma profile across the bow shock . . . . . . . . . 102

8.2 Sketches of the solar wind coordinate system . . . . . . . . . . . . . 104

8.3 Cluster orbit in the solar wind coordinate system . . . . . . . . . . . 105

8.4 Wave power in frequency domain . . . . . . . . . . . . . 106

8.5 Magnetic field and ion number density profile . . . . . . . . . . . . . 106

8.6 Wave power in $k$-space . . . . . . . . . . . . . . . . . . 107

8.7 Histograms of frequencies . . . . . . . . . . . . . . . . . . 108

8.8 Histograms of wave numbers divided by ion inertial wave number . . . . 109

8.9 Histograms of wave numbers divided by thermal ion gyro-wave number . 110

8.10 Histograms of phase velocities divided by fast mode speed . . . . . . . 110

8.11 Histograms of phase velocities divided by intermediate mode speed . . . 111

8.12 Histograms of phase velocities divided by slow mode speed . . . . . . . . 111

8.13 Histograms of propagation angles . . . . . . . . . . . . . . . . 112

8.14 Histograms of magnetic polarization . . . . . . . . . . . . . . . . 112

8.15 Histograms of phase angles between plasma density and magnetic field strength variations . . . . . . . . . . . . . . . . . . . . 113

8.16 Histograms of phase angles between plasma bulk velocity and magnetic field variations . . . . . . . . . . . . . . . . . . . . . . . . . . . 113

8.17 Distributions of mean magnetic field, plasma density and temperature . . 114

8.18 Distributions of propagation angles, phase velocities, and phase angles between plasma density and magnetic field variations . . . . . . . . . . . 115

8.19 Spatial distribution of phase velocities in the GSE coordinate system . . . 117

8.20 Spatial distribution of phase velocities in the solar wind system . . . . . . 118

8.21 Sketch of propagation pattern . . . . . . . . . . . . . . . . . . . . . 119

8.22 Histograms of relative errors . . . . . . . . . . . . . . 120

9.1 Dispersion relation and $k$-spectra . . . . . . . . . . . . . . . . . 133

9.2 Comparison of $k$-spectra between direct and indirect measurements . . . 135 
9.3 Dispersion relation and $k$-spectra for non-compressive components . . . 136

9.4 Dispersion relation and $k$-spectra for compressive components . . . . . 137

9.5 Probability distribution functions . . . . . . . . . . . . . . . . . 138

10.1 Cluster magnetic field investigators . . . . . . . . . . . . . . . . 160 



\section{List of Tables}

6.1 Time intervals and frequencies of foreshock waves $\ldots \ldots$. . . . . . 88

7.1 Plasma parameter $\beta$, temperature anisotropy, and transport ratio . . . . . 97

8.1 Time intervals and frequencies of magnetosheath waves . . . . . . . . . . 123

8.2 Time intervals and frequencies of quasi-perpendicular shock upstream waves . . . . . . . . . . . . . . . . . . . . . . . . . . . . . 129 



\section{Summary}

Studying space plasmas is of significant importance not only in geophysical or space research, but also with respect to the fundamental physics of collisionless plasmas. It has been suspected since the days of the early development of plasma physics that collisionless plasmas exhibit numerous kinds of waves and instabilities, but it is rare to unambiguously identify them in experiments in spite of a number of in-situ spacecraft measurements for decades. Our knowledge about space plasmas is still limited. One of the difficulties in understanding space plasma phenomena stems from the fact that the experiments were made using single or at best double spacecraft measurements. It is not easy in space unless having any assumptions to distinguish spatial variations from temporal variations from only one or two point measurements. Spatial scales like wavelengths are not yet investigated in space. It was not until the advent of Cluster, four spacecraft mission, that one measures space plasma at last with spatial resolution in three dimensions.

The solar wind interacts with the Earth's magnetic field in various ways including waves. The existence of waves upstream and downstream of the bow shock ahead of the Earth have been known since the discovery of the bow shock and various kinds of plasma models and ideas were proposed to understand the nature of these waves. Studying the Earth's bow shock and its associated wave activity is important, for it is the only accessible collisionless shock for detailed investigations and has immediate astrophysical implications.

This $\mathrm{PhD}$ thesis presents the analysis of low frequency waves upstream and downstream of the terrestrial bow shock, taking advantage of the four point measurements, and contributes to fundamental plasma physics as well as the research of Sun-Earth interaction. The thesis first reviews our current understanding about the bow shock, upstream and downstream waves and turbulence in Chapter 2 and introduces the experiment using Cluster and wave analysis methods in Chapter 3.

Chapter 4 presents a dispersion analysis of the upstream waves. The wave dispersion relation is determined experimentally and shows a good agreement with the one calculated for the ion beam plasma model, suggesting that the upstream waves represent whistler and beam resonant waves. This is one of examples of wave mode identification and confirms the physical processes drawn by the earlier studies that some upstream ions are specularly reflected at the shock and flow against the incoming ions, while they form an unstable particle distribution in velocity space which drives waves and collapses into a stable state.

Chapter 5 presents an example of wave-particle interaction. The dispersion and polar- 
ization analysis indicate the existence of the whistler wave in the upstream region. The wave is accompanied by nongyrotropic electrons which are trapped by the wave field. While it is known that some beam ions are phase bunched by the wave field, there is also an interplay between the waves and the electrons.

Chapter 6 presents a statistical study of the upstream waves. It is shown that the wave phase velocities in the plasma rest frame of reference are oriented toward upstream along the magnetic field. This direction is the same as that of the backstreaming ions.

Chapter 7 presents the dispersion analysis of the downstream waves. The dispersion curves are investigated along a Cluster orbit and show a transition from the whistler and the beam resonant waves in the upstream region to the mirror mode in the downstream region. The upstream waves are not transmitted across the shock, as they are swept by the solar wind toward downstream.

Chapter 8 presents a statistical study of the downstream waves. While the upstream waves propagate parallel to the background magnetic field, the downstream waves propagate perpendicular. The mirror mode properties are frequently detected in the downstream region but they have finite propagation speed, possibly coupled to the background inhomogeneities or nonlinear effects. On the statistical average there is an organization in wave propagation pattern: outward divergent in the upstream region; toward the magnetosheath flank region aligned with the plasma flow direction in the downstream region; and inward convergent in the magnetosheath flank.

Chapter 9 attempts to determine the spectra of magnetic turbulence directly in the wave number domain. The direct determination has been done for the first time in space plasma. The spectra for the upstream waves show three ranges: the injection range identified at small wave numbers with the wave coupling region between the whistler and the beam resonant mode wave, the quasi-inertial range, and the dissipation range. The fluctuations exhibit properties of not fully developed turbulence but intermittency, suggesting that there is not enough time for turbulence to become fully developed.

The above results indicate that disturbances in the plasma caused by the bow shock lead to wave excitation both in the upstream and the downstream regions, but the wave properties are different between them and accordingly the physical processes are different. The upstream waves propagate parallel to the magnetic field and are identified as the one driven by the ion beam instability, while the downstream waves propagate perpendicular and represent the mirror modes. On the other hand they exhibit a unique propagation pattern imposed by the background magnetic field topology in those regions. Multi-point measurements are essential in understanding waves, instabilities, and turbulence in space. 


\section{Introduction}

\subsection{Space plasma}

A plasma is an ionized gas and often called the fourth state of matter. It is realized when the temperature of the matter is so high that the atoms dissociate into ions and electrons. The plasma behaves considerably different from what we know about the matter on the ground of the Earth. Since it is an electrically conductive medium and capable of carrying electric currents, it is not simply described by the laws of gas or fluid dynamics. It reacts sensitively to electric and magnetic fields and disturbs those fields due to the currents in it which complicates us to comprehend its behavior correctly. With the advent of the spaceflight era the interests of geophysicists are oriented not only into the Earth's internal structure and its dynamics but also the neighboring environment about the Earth and it was understood that the neighborhood is in an ionized state. Today it is widely believed that most of the baryonic matters in the Universe is in the plasma state. Space physics is therefore to a large part plasma physics.

There are different kinds of plasmas. The plasmas are characterized into, for example, collisional or collisionless plasmas, dense or dilute plasmas, hot or cold plasmas, magnetized or unmagnetized plasmas, fully or partially ionized gas, and so on. To specify the plasma temperature, the plasma parameter $\beta$ is often introduced which is a ratio of thermal to magnetic field energy density. What characterizes the space plasma uniquely is the absence of particle collisions. Too huge system size and too small particle density make it hard to achieve laboratory experiments on the ground. Instead, the use of spacecraft has been enabling the physicists to make in situ experiments in the near Earth space, which is the only accessible collisionless plasma to us. One may regard that the near Earth space plasma serves as a natural laboratory and expect to apply the results to astrophysical phenomena.

Plasmas in space are in many situations accompanied by electromagnetic fields. Plasmas are electrically quasi-neutral, as the electric charge in each volume element is shielded by oppositely charged particles. But electric fields may arise in plasmas, for example, when plasmas flow in magnetic fields, they generate convective electric fields. Magnetic fields may also arise due to so-called dynamo processes. Magnetic fields in the plasma are subject to two effects, depending on the electric conductivity of the plasma. They diffuse under the low conductivity; or they are "frozen-in" to the plasma under the high conductivity, which means that the fields are carried by the plasma flow. For instance, 
the plasma in the interplanetary space is characterized by a very high conductivity and the interplanetary magnetic field is carried by the solar wind plasma away from the sun. In space it is possible that the energy density of electromagnetic fields exceeds kinetic or thermal energy of the plasmas. Furthermore, plasmas allow processes that exchange different kinds of energies between one another. The kinetic energy can be converted into the magnetic field energy by the dynamo processes, and on the other hand the magnetic field energy can be converted back into the kinetic energy by the reconnection processes. The electromagnetic fields play therefore an important role as well as the plasma itself.

\subsection{Waves and instabilities}

The collisionless nature of space plasma indicates that unstable configurations in the sense of ordinary gas dynamics such as the temperature anisotropy, beams, and temperature difference among species are preserved in principle. Charged particles, however, interact with one another remotely via disturbances of the electric and the magnetic fields. The space plasmas exhibit in many cases dynamic and transient phenomena. As they are electrically conducting, their dynamic motions are accompanied by magnetic field fluctuations. While an ordinary gas permits solely sound waves propagating in the medium, plasmas exhibit numerous kinds of waves propagating. Electric currents in the plasma react to the wave electric and magnetic fields in various ways depending on scales and frequencies of interest, which results in distortion or introduction of restoration force. The plasmas also allow many kinds of instabilities to exist, in which case the restoration force in the disturbance reacts positively in such a way that the disturbance grows. The plasmas can distribute energy and momentum to bring the system toward an equilibrium by means of waves and instabilities.

\subsection{Shock waves}

Boundaries are often formed in space when different kinds of plasmas meet each other. For example, the interaction of the solar wind with the Earth's magnetic field results in the formation of a magnetospheric cavity bounded by the magnetopause. The solar wind is a plasma stream arising from the solar corona. Since the solar wind flows faster than any wave propagation speeds allowed, a standing shock wave (bow shock) is formed ahead of the Earth as it meets the Earth as an obstacle. The interaction also results in the formation of a boundary separating the solar wind plasma from the Earth ionospheric and magnetospheric plasmas called the magnetopause. Other types of boundaries may exist in the plasma in general. They are classified based on the conservation laws across discontinuities in the ideal magnetohydrodynamical picture (the Rankine-Hugoniot relations). The shock wave solution is also derived from the Rankine-Hugoniot relations. In the gas dynamics a shock wave comes about when a supersonic flow meets an obstacle and particles collide suddenly. A similar situation can be found in the plasma though particles remain 
collisionless. For this to happen, the flow speed must exceed not the sound speed but the magnetosonic speed which is determined by the magnetic field, the plasma density, the temperature, and the propagation direction. A sudden compression of the magnetic field and the plasma is accompanied when the plasma undergoes the shock. Structure, dynamics of the shock, and dissipation process are known to be sensitively dependent on the upstream states such as the magnetic field geometry, the upstream flow velocity, and the plasma parameter $\beta$. The collisionless shocks under some conditions are markedly characterized by a specular reflection of particles at the shock such that a portion of the upstream particle population gains energy at the shock and streams against the incoming flow toward upstream. The backstreaming particles, while interacting with the upstream flow, form a unique region ahead of the shock called the foreshock. Such a situation is never found in the ordinary gas dynamics nor in the fluid dynamics.

\subsection{Upstream and downstream waves}

Since the first spacecraft detected the existence of the shock wave ahead of the Earth it has been known that the upstream and the downstream plasmas exhibit moderate to high level of magnetic field and plasma fluctuations, indicating that the shock formation is accompanied by some wave processes ahead of and behind the shock. Many theoretical and experimental attempts have been made to understand the physics of the upstream and the downstream waves. However, it is not easy to investigate wave properties in a proper frame of reference, the plasma rest frame, because one can not distinguish between spatial and temporal variations from the acquired spacecraft data. This problem reflects the fact that the waves are Doppler shifted as the background medium sweeps the waves and modulates wave frequencies. Using single spacecraft, it is impossible to determine how much the frequency detected comes from the convection part and how much from the intrinsic one. There were double spacecraft missions but only one dimensional spatial scale was obtained and one must have a good condition that the wave propagation is aligned with the spacecraft separation direction.

Acquisition of spatial resolution in three dimensions have remained as a task ever since the earliest spacecraft missions. With the advent of the Cluster mission, consisting of four spacecraft, the spatial resolution has at last become available at in situ observations. Having speculations and scenarios derived from the early spacecraft observations and theoretical studies in mind, this thesis aims to reveal the nature of the upstream and the downstream waves.

This thesis is organized in the following fashion. Chapter 2 introduces the concept of a collisionless shock. Foundations of the shock, theories, and observations about the upstream and the downstream waves are reviewed. Chapter 3 describes an outline of the Cluster mission and instrumentation for the magnetic field and the plasma measurements. 
The wave analysis methods are presented, too. Chapter 4 and 5 present dispersion analyses of the upstream waves, where wave dispersion relations are determined experimentally and compared to theoretical dispersion curves. Chapter 6 presents a statistical study of the upstream waves. Chapter 7 presents a dispersion analysis of the downstream waves. The dispersion curves are investigated along a spacecraft orbit and compared with the upstream case. Chapter 8 presents a statistical study of the downstream waves. Chapter 9 attempts to determine directly turbulence spectra of magnetic field fluctuations for the upstream waves. 


\section{Shocks, upstream and downstream waves, turbulence}

\subsection{Collisionless shocks}

Most of our everyday notions of the nature of shock waves (or simply shocks) come from our experience of supersonic aircraft or explosive blasts. The study of shocks began with ordinary gas dynamics in the late 19th century and reached its maturity during the 1940s, at the time of the development of high-performance aircraft. The study of plasma shocks surfaced during the 1950s. Earlier, it was debated whether collisionless shock waves even existed. Some argued that the rarity of collisions in a high temperature plasma precluded the existence of shocks, while others maintained that collective micro-turbulence would replace particle collisions to create a shock with a thickness much less than a collision mean free path. The solar wind proved, upon its discovery in 1959-1963, to have a fast flow speed and an enormous mean free path which is comparable to the distance from the Earth to the sun. Since it had been difficult to make collision-free plasmas in the laboratory, some foresaw that the first collisionless shock would be discovered in space. And so it was, standing in the solar wind in front of the Earth's magnetosphere called the bow shock (Sonett and Abrams 1963, Ness et al. 1964). Also, high-altitude nuclear explosions in the upper atmosphere and magnetic pinch fusion research motivated laboratory investigations of the collisionless shocks. There was a good collaboration between laboratory and space experimentalists, theoreticians, and specialists in numerical simulation. After the collaboration ended in 1970s, when the interest in the magnetic pinch fusion waned and financial support for the laboratory experiments disappeared, the space community was left to its own devices. Nowadays the space community plays a dominant role in the collisionless shock research. The ISEE spacecraft (Ogilvie et al. 1977) was well suited to detailed research of the collisionless shocks. The use of two spacecraft made it possible to measure scale length, easy to do in laboratories but difficult in space. The discovery of the Earth's bow shock and the subsequent observations of planetary bow shocks and interplanetary shocks have now firmly established that shocks can be produced in the collisionless plasma. Space plasmas exhibit both stationary shocks like bow shocks produced by the solar wind interaction with planets and transient shocks like solar flares and supernova explosions.

Formation of a shock can be explained in terms of steepening of a large amplitude 
wave. Such a wave is nonlinear, behaves like a soliton, and can steepen because the wave speed with a shorter wavelength could be changed by the wave itself, which becomes the basis of the shock. Nonlinear waves generally bear some resemblance to more familiar linear modes and have a velocity typically equal to that of their corresponding linear mode and a wavelength at which the wave mode becomes dispersive (Krall 1997). This would eventually lead to sharp gradients and shock formation. When dissipation is present, the solitons interact with the background medium as they propagate, so that the background state is changed by the solitons. For a shock to be stable, the wave steepening must be balanced against the dissipation.

In collision dominated gases, the shock wave forms when the relative speed between the flow and the obstacle exceeds the sound speed. The flow energy is dissipated by binary collisions which lead to viscosity and friction. The ratio of the flow speed to the sound speed is called the Mach number, $M$. The upstream region is characterized by $M>1$ (supersonic flow) and low entropy, while the downstream region is characterized by $M<1$ (subsonic flow) and high entropy, density, and pressure. The transition across a shock occurs in a distance of the order of a few collision mean free paths. In a collisionless plasma such as the solar wind the distance of even a few collisional mean free paths is very large, and other processes intervene to control the thickness of such shocks. Energy and momentum can be transferred among particles via electric and magnetic field oscillations. These collective motions add to a rich variety of possible shocks and dissipation mechanisms. For instance, at the larger Mach numbers, thermal spread in the plasma allows particle reflection at the shock, which provides an additional dissipation mechanism. The situation becomes further complicated due to the fact that there are several propagation speeds allowed in the plasma. In magnetohydrodynamics (MHD), the one-fluid picture of plasmas, there are three fundamental waves: fast, intermediate (Alfvén), and slow modes. Because they have different characteristic propagation speeds, there are different shocks which inherit some properties of corresponding wave modes. The structure, dynamics, and dissipation mechanism of the collisionless shocks are generally dependent on the magnetic field geometry with respect to the shock normal, the Mach number, and the plasma parameter $\beta$ (ratio of thermal to magnetic pressure).

\subsubsection{Rankine-Hugoniot relations}

No matter how a shock is structured and organized, one knows that mass, energy, and momentum must be conserved. One can then apply the conservation laws of MHD to relate the downstream state with the upstream one and find possible solutions of the shocks. In the case of an ordinary gas, these relations were first derived by Rankine and Hugoniot toward the end of 19th century. The Rankine-Hugoniot relations determine uniquely the downstream state in terms of the upstream states. Thus the shock structure is determined only by the dissipation mechanism, viscosity, which produces a shock with a thickness of a few mean free paths. In the case of a collisionless plasma, the conservation laws (also called the Rankine-Hugoniot relations) do not provide a unique relationship between the 


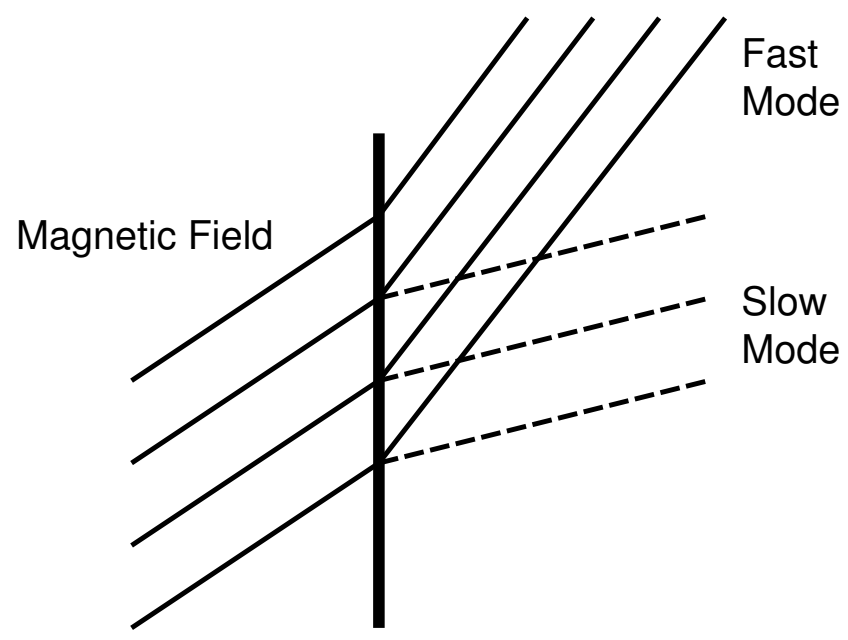

Upstream

Shock

Downstream

Figure 2.1: Sketch of magnetic fields across a fast and a slow shock wave (after Baumjohann and Treumann 1997).

upstream and the downstream state. The Rankine-Hugoniot relations allow generally not only shocks but also discontinuities to exist (Baumjohann and Treumann 1997).

As mentioned above, three kinds of shocks exist from the viewpoint of the MHD conservation laws: fast, intermediate, and slow shocks. The fast and the slow shock have the same behavior in terms of the plasma and magnetic pressure as the corresponding fast and slow mode waves in MHD. Across the fast shock, the magnetic pressure increases together with the plasma pressure. The normal component of the magnetic field to the shock is constant and the increase is seen only in the transversal component. Therefore, the downstream magnetic field is bent away from the shock normal (Fig. 2.1). In contrast, the magnetic pressure downstream of the slow shock decreases and the field is bent toward the shock normal. Observationally, the fast shocks are the most frequent type in the solar system such as planetary bow shocks and interplanetary shocks. The slow shocks are rarer, but they have been observed and appear in some theories of magnetic reconnection.

The fast and slow shocks exhibit an interesting property called the coplanarity theorem that the upstream and downstream magnetic field directions and the shock normal all lie in the same plane (Baumjohann and Treumann 1997). The intermediate shock is a special case. In an isotropic plasma it is not a shock but called a rotational discontinuity, across which there is a flow but no compression of the plasma or dissipation. Our discussion later concentrates on the fast shocks. 

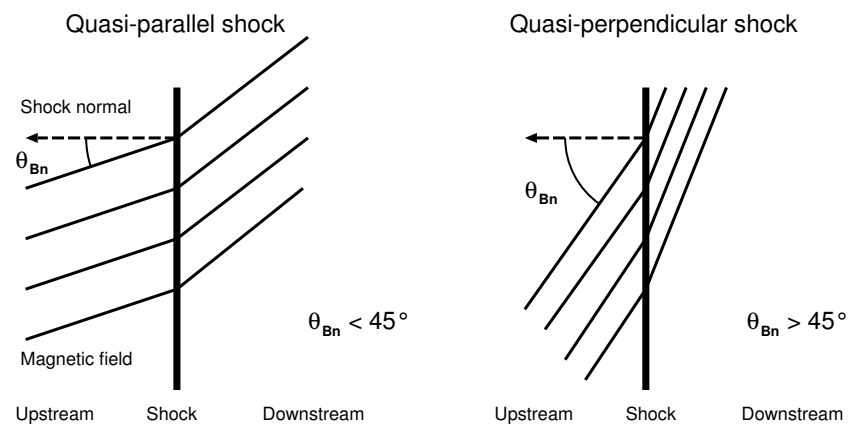

Figure 2.2: Sketch of magnetic fields across a quasi-parallel and a quasi-perpendicular shock (after Baumjohann and Treumann 1997, Schwartz 2000).

\subsubsection{Different types of shocks}

There are many different types of shocks even if we restrict ourselves to the fast shocks. At all planetary bow shocks we find different structures. Early on there were debates whether or not such shocks were actually stable. Perhaps all the different profiles seen in observations were just fleeting glimpses of an ever-changing entity. A major contribution from the observations was the demonstration that there was a definite pattern of shocks determined by the complete set of upstream parameters. The most important factor in controlling the type of shock is the direction of the upstream magnetic field relative to the shock normal. Depending on the value of $\theta_{B n}$ which is the angle between the magnetic field direction and the shock normal, shocks are classified as parallel shocks $\left(\theta_{B n}=0^{\circ}\right)$, as perpendicular shocks $\left(\theta_{B n}=90^{\circ}\right)$, or as oblique shocks $\left(0^{\circ}<\theta_{B n}<90^{\circ}\right)$. One also speaks of quasi-parallel shocks $\left(\theta_{B n}<45^{\circ}\right)$ and quasi-perpendicular shocks $\left(\theta_{B n}>45^{\circ}\right)$. Fig. 2.2 shows the quasi-parallel and the quasi-perpendicular shock. The distinction between the two shocks is physically relevant. The perpendicular shocks are based on the fast magnetosonic waves, and the parallel shocks are based on the whistler waves (Krall 1997). When the plasma becomes so hot that the magnetic field can be ignored, both the waves degenerate into the ion acoustic wave and shocks become the hydrodynamic shock.

Particle dynamics is as important as the fluid picture to understand the shocks. The ions and electrons encountering the compressed magnetic field at the shock have different gyroradii. The ions can penetrate deeper into the field than the electrons, because the ions have larger mass. This difference in penetration depth generates a charge separation electric field in the shock normal direction, pointing toward upstream. The electric field then reflects a number of ions back into the upstream region, while it attracts and captures the electrons.

Shocks also depend on the Mach number and the plasma $\beta$. In the collisionless plasma, shock heating must use mechanisms unique to plasmas. In the case of the low 
Mach number shocks (called subcritical shocks), mixing of the upstream flow and the plasma reflected from the obstacle causes a beam-beam instability. This results in Joule heating due to the generation of anomalous collisions via field oscillations and resistivity in the current layer inside the shock. The heat is produced depending on the current in the plasma. The instability and field fluctuation also cause an effective viscous interaction in the shock front and produce heat depending on the velocity gradient. In both cases waves and instabilities replace collisions to scatter particles. These processes run relatively slowly. When the Mach number is higher than a critical Mach number $M_{c} \approx 2.7$, there is not enough time for the heat production and braking the flow speed. In such a case (supercritical shocks) the dissipation is provided by a specular reflection of some of ions and electrons at the shock (Kennel et al. 1985). Since not the whole flow is reflected, these particles do not carry magnetic flux back upstream. The ion reflection plays a dominant role rather than the electrons for the shock transition process, because they carry most of mass, momentum, and energy.

\section{Quasi-parallel shocks}

The parallel shock has the upstream magnetic field parallel to the shock normal and the direction of the field is unchanged by the shock, because the magnetic field variations appear only in the transversal component across the shock. The total magnetic field is also unchanged. There is a compression in the plasma but not in the field. From the MHD perspective, this means that the shock behaves like the one in an ordinary gas, where the magnetic field does not play a role. However, in the context of a collisionless plasma the only way for dissipation to occur is the field-particle processes and the strict parallel shock is never realized. Realistic parallel shocks are always quasi-parallel and react magnetically.

The quasi-parallel shocks are highly oscillatory to large distances in front of the shock called foreshock. Fig. 2.3 top illustrates a magnetic field pattern across the quasi-parallel shock. The quasi-parallel shocks allow the reflected ions to escape from the shock into the foreshock along the magnetic field. As the reflected ions flow against the incoming plasma, they drive the ion beam instabilities. These instabilities excite large amplitude waves in the upstream region. As the upstream waves are convected back to the shock due to a large upstream flow speed, they steepen up and the shock re-forms itself.

\section{Quasi-perpendicular shocks}

The upstream magnetic field is perpendicular to the shock normal at the perpendicular shock. The typical quasi-perpendicular shock profile consists of the upstream and downstream regions connected by a steep shock ramp and accompanied by a shock foot region in front of the ramp, where the magnetic field gradually rises. The shock ramp exhibits a magnetic shock overshoot before settling at the average magnetic field strength behind the shock (Fig. 2.3 middle). From the MHD perspective, there is a limit of the jump of the magnetic field and density at a high Mach number shock. These quantities change at most by a factor 4 across the shock, assuming the ratio of specific heat $\gamma=\frac{5}{3}$ (Burgess 1995). 


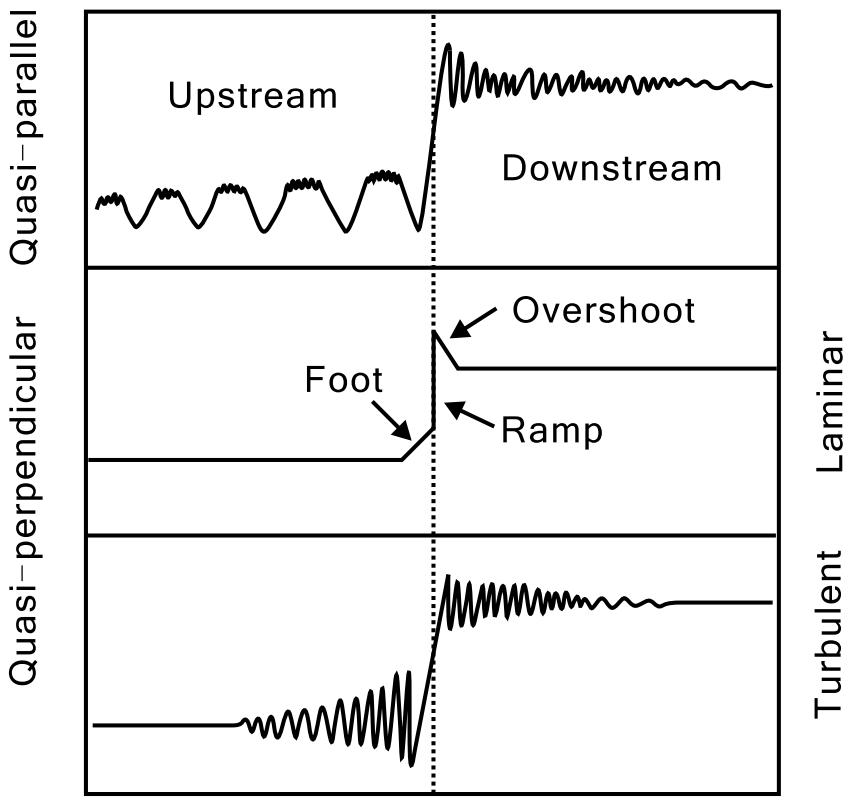

Figure 2.3: Typical magnetic field profiles across shocks: across a quasi-parallel shock (top), across a laminar quasi-perpendicular shock (middle), and across a turbulent quasiperpendicular shock (bottom, after Baumjohann and Treumann 1997).

At the quasi-perpendicular shocks, the main transition from the upstream to the downstream plasma is accomplished at the sharp ramp. The reflected ions gyrate back to the shock and enter the downstream region. The foot region is produced by the reflected ions and subject to various instabilities with enhanced level of low frequency magnetic field fluctuations. The gyration of the reflected ions results also in the temperature anisotropy ( $T_{\perp}>T_{\|}$, where $T_{\perp}$ and $T_{\|}$denote perpendicular and parallel temperature to the magnetic field, respectively) and lead to the excitation of anisotropy-driven instabilities (Sckopke et al. 1990). When the Mach number becomes large, the character of the shock transition changes from the laminar to the turbulent one (Fig. 2.3 middle and 2.3 bottom).

\subsubsection{Bow shock}

The most famous example of the shocks is the Earth's bow shock (Fig. 2.4). It develops as a result of the interaction of the solar wind with the Earth's magnetosphere. The solar wind is a flow of magnetized plasma originating from the solar corona and characterized by the super fast-magnetosonic speed (typically at about $400 \mathrm{~km} / \mathrm{s}$ ) and the low density 


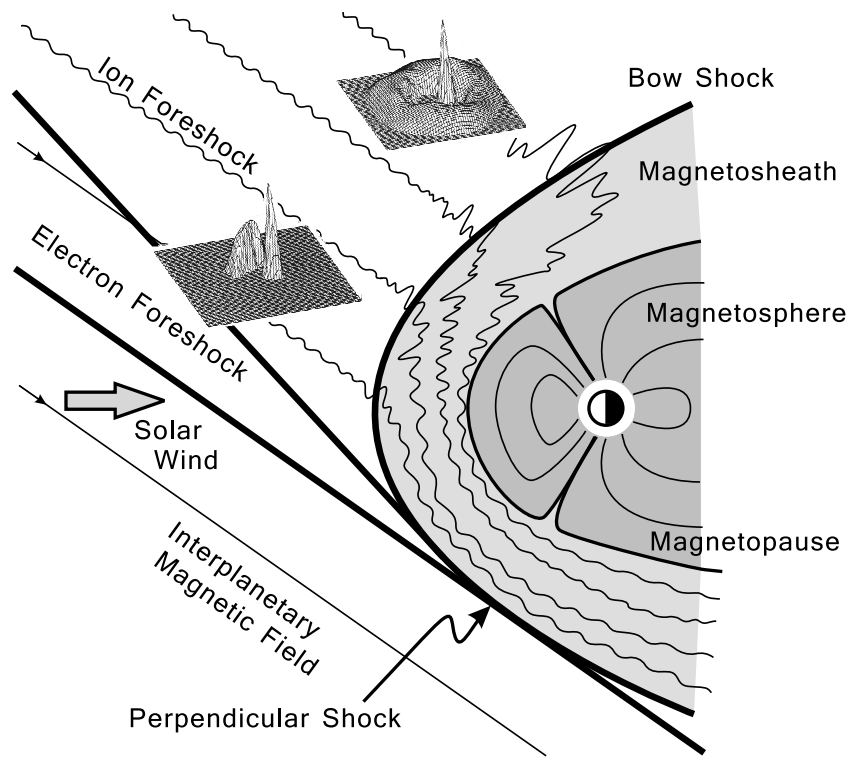

Figure 2.4: The Earth's bow shock and magnetosphere in the magnetized solar wind plasma flow. Shown are the magnetic field fluctuations across the shock; counterstreaming (near the center) and ring distribution of ions in velocity space (top, after Treumann and Scholer 2001).

(about $7 \mathrm{~cm}^{-3}$ ) at the Earth's orbit. The magnetic field of the solar wind is called the Interplanetary Magnetic Field (IMF). The magnetosphere brakes the solar wind flow. The magnetosphere is a blunt obstacle at rest, and bounded by a discontinuity of plasma called the magnetopause. The distance of the dayside magnetopause is typically about $10 R_{E}$ $\left(1 R_{E}=6370 \mathrm{~km}\right)$ from the Earth and the one of the bow shock is about $15-20 R_{E}$. Across the bow shock the solar wind becomes denser, decelerated, and heated, accompanied by the enhanced magnetic and plasma pressure. Since the solar wind is a stream with a high magnetosonic Mach number $\left(M_{m s} \approx 8\right)$, the bow shock is a fast magnetosonic shock and the solar wind speed changes from a super- to a sub-magnetosonic speed across the shock. The shocked solar wind plasma flows about the Earth's magnetosphere and the region bounded by the bow shock and the magnetopause is called the magnetosheath. Curved shocks like the bow shock can always be divided into regions of the quasi-parallel and the quasi-perpendicular shocks. The bow shock is an ideal object to study the physics of collisionless shocks. Recently, the Hubble Space Telescope observed a bow shock in the Orion Nebula (Fig. 2.5). Studying the Earth's bow shock has an immediate implication to astrophysical shocks. 


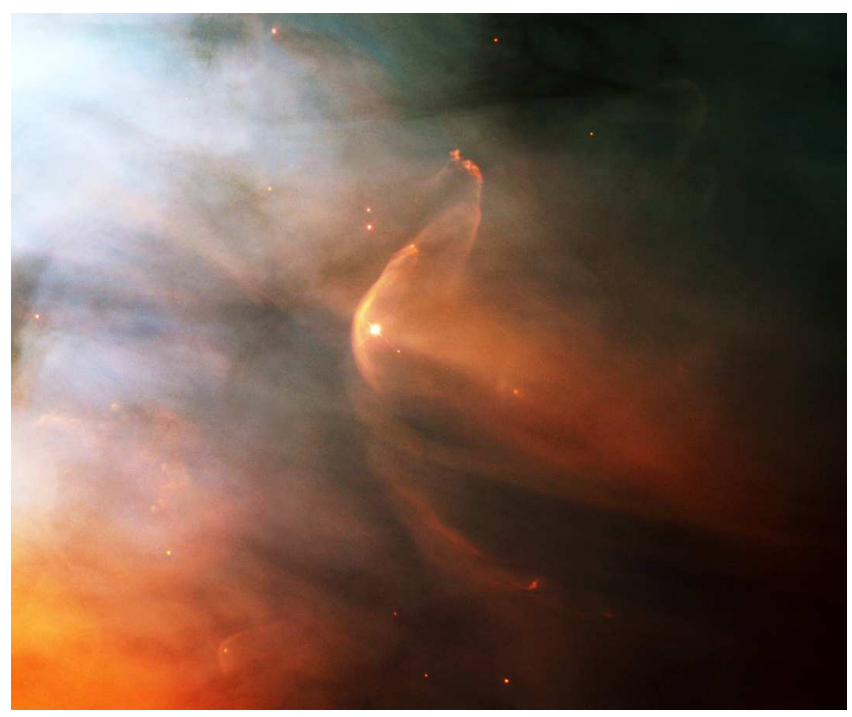

Figure 2.5: The glowing arc taken by the Hubble Space Telescope is a bow shock caused as a young star (LL Ori, in the middle) ploughs through the gas of the Orion Nebula. The star emits a vigorous wind, a stream of charged particles moving rapidly outward. The material spewed from LL Ori collides with slow-moving gas evaporating away from the center of the Orion nebula, located to the upper left of the image. The surface where the two winds collide is seen as the crescent-shaped bow shock. The arc is several hundred times bigger than the entire solar system (Courtesy of NASA and the Hubble Heritage Team of STScI/AURA).

Some of ions and electrons are reflected at the bow shock and escape into the solar wind, while they undergo the solar wind convective electric field and drift in the antisunward direction. These escaping particles warn the solar wind about the existence of an obstacle and brake the solar wind before it reaches at the shock. In principle, a foreshock already belongs to the shock transition. The foreshock region is further divided into two zones, the electron foreshock and ion foreshock (Fig. 2.4). The electron foreshock is a narrow region, bounded on one side approximately by the magnetic field line tangential to the shock. It contains electrons which have been specularly reflected at the shock or heated in the shock ramp. Some electrons have sufficiently large field-aligned velocities to escape into the solar wind and they travel far along the tangential field line. They excite Langmuir and upper-hybrid waves, become slowed down, and are scattered into an isotropic distribution (Treumann and Baumjohann 1997). The ion foreshock forms a larger angle with respect to the tangential field line than the electron foreshock, because the velocity 
of the backstreaming ions is much lower than that of electrons. The backstreaming ions lead to an unstable velocity distribution function together with the ambient solar wind ions and the distribution collapses into the ring distribution, while exciting waves via the ion beam instabilities (Paschmann et al. 1979, 1981).

Downstream of the shock, the magnetosheath plasma parameters show a large scale spatial organization imposed by the shape of the magnetopause. Because the physical processes of the shock depend on the orientation of the IMF, the properties of the magnetosheath plasma just behind the bow shock depend also on whether the shock is quasiperpendicular or quasi-parallel. In general, the magnetosheath tends to be in a more turbulent state behind the quasi-parallel shock than the quasi-perpendicular shock. The magnetosheath plasma is characterized as follows: (1) Average density and magnetic field strength are higher than that of the solar wind by a factor consistent on average with the Rankine-Hugoniot relations for the fast mode shock (up to 4); (2) The average flow direction deviates from the sun-Earth direction and the plasma flows about the magnetosphere; (3) The flow velocity is lower than the local fast magnetosonic speed; (4) The flow velocity increases again up to the near magnetosonic speed around the magnetopause flanks; (5) The ion temperature does not increase very much over its upstream value, such that the ion to electron temperature ratio in the magnetosheath is of the order of $6-7 ;(6)$ The plasma $\beta$ shows large variations from the order of unity to values much greater than one; (7) The magnetosheath plasma develops a temperature anisotropy $\left(T_{\perp}>T_{\|}\right)$behind the bow shock that increases toward the magnetopause. The anisotropy is more prominent in the ions than in the electrons. It arises from adiabatic heating in the perpendicular direction as the plasma and magnetic field are compressed toward the magnetopause, or from the ion reflection at the shock.

\subsection{Upstream waves}

Early single spacecraft observations already indicated the existence of ion distributions upstream of the bow shock that could not be classified as being either solar wind like or magnetosheath like (Asbridge et al. 1968), accompanied by the enhanced magnetic field fluctuations (Greenstadt et al. 1968). It was shown that these fluctuations were in fact quasi-monochromatic waves with periods of about 30 seconds and typically left-handed in the spacecraft frame (Fairfield 1969). Also observed were linearly polarized steepened waves, termed shocklets, associated with discrete wave packets (Russell et al. 1971) and $1 \mathrm{~Hz}$ waves (Fairfield 1974).

It was proposed that the backstreaming ions were responsible for the generation of the low frequency waves (Barnes 1970). However, to test such a model experimentally, the properties of waves must be investigated in the plasma rest frame. Minimum variance analysis could be applied to single spacecraft observations and used to compute the direction of wave propagation, with a $180^{\circ}$ ambiguity, but it was not possible to determine wave phase speeds, exact directions of propagation, and wavelengths. 
A large part of our knowledge about the foreshock owes a lot to the ISEE double spacecraft observations. Ion distributions could be identified into different types (Gosling et al. 1978): reflected, intermediate, and diffuse (Paschmann et al. 1979, 1981). The low frequency waves were shown to be associated with the diffuse ions, but not with the backstreaming beam ion distributions. It was also shown that the observed waves were left-hand polarized in the spacecraft frame but intrinsically they were right-hand polarized and propagated in the solar wind frame (plasma rest frame) away from the shock and in the direction of the backstreaming ion beams (Hoppe et al. 1981, Hoppe and Russell 1983). To explain the observed phenomena, it was thought that the reflected ions generated the fast magnetosonic waves, creating 30s waves and intermediate distributions. Further wave-particle interaction would then result in the hot diffuse distributions associated with the shocklets and the discrete wave packets. Consequently, the foreshock waves were identified as being intrinsically right-handed and qualitatively consistent with the generation by backstreaming ions through the right-hand resonant ion beam instability (Gary 1991, 1993). Fig. 2.4 shows schematically the upstream waves and the ion velocity distributions. Near the shock and the tangential field line the backstreaming ions form a beam distribution in the magnetic field direction. As they are swept by the solar wind, they excite waves and collapse into a ring distribution.

\subsubsection{Ion beam instabilities}

The ion beams propagating along the magnetic field generate low-frequency electromagnetic waves through the ion beam instabilities. The beam is less dense than the background ions but becomes warmer due to scattering at the self-generated waves. There are three types of the beam instabilities: the right-hand resonant, the left-hand resonant, and the non-resonant beam instabilities. Let us assume that the plasma consists of three populations, a hot Maxwellian electron distribution and two drifting Maxwellian ion distributions, a denser core distribution and a dilute beam distribution. When the ion beam is cool, only the beam is resonant and both the electrons and the core ions do not satisfy the resonant condition. The resonant condition for the beam with the right-hand mode is

$$
\omega=k_{\|} v_{b}-\Omega_{b},
$$

where $\omega$ denotes the wave angular frequency, $k_{\|}$the wave number parallel to the magnetic field, $v_{b}$ the beam velocity relative to that of the core ion distribution, and $\Omega_{b}$ the cyclotron frequency of the beam ions. This condition corresponds to a situation in which the beam ions see a constant electric field in its own frame of reference, so that it can exchange a significant amount of energy with the wave. This instability excites essentially a righthand whistler wave with positive helicity propagating along the beam. At low frequencies the wave becomes the fast magnetosonic wave (Gary 1986).

The resonance with the left-hand polarized mode is also excited, which becomes the ion whistler or ion cyclotron mode at long wavelengths. It has negative helicity and propagates parallel to the beam. However, it is easier to excite the right-hand mode under the 
cool beam condition because at the low thermal velocities of the beam there are only few ions which can resonate with the left-hand mode. Therefore cold beams will predominantly generate the right-hand waves.

The last example, the non-resonant mode, excites waves propagating in the opposite direction to the ion beam. It has negative helicity and small phase velocity. The instability is basically a firehose instability caused by the inertia of the fast ion beam which exerts a centrifugal force on the bent magnetic field and excites a wave at very low frequencies close to zero. The instability has a larger threshold, since it has to overcome the restoring forces of perpendicular pressure and magnetic tension. The solar wind ions are cool and the right-hand mode instability is the fastest growing mode in the foreshock. Scattering of the ion beams by the broad-band electromagnetic waves heat the ion beams diffusely, while in monochromatic waves the beams become partially trapped and thus phase bunched (Thomsen et al. 1985). Both effects have been observed. In addition, the waves may reach such large amplitudes that nonlinear effects appear. As the large amplitude waves are convected downstream toward the shock, they steepen, accumulate at the shock front and modify it.

Recently it was understood that the ion beam plasma is unstable when treated linearly but can evolve into a steady nonlinear configuration in which wave numbers become a complex number and hence a spatially damping oscillatory wave packet appears called oscilliton (Sauer et al. 2001, Sauer and Dubinin 2003, Dubinin et al. 2004). Such a wave packet is provided by the linear instability, and the nonlinear structure is sustained by the momentum exchange between the two different ion populations mediated by the magnetic field stress.

\subsubsection{Shock foot waves}

At the quasi-perpendicular shocks $\left(\theta_{B n}>45^{\circ}\right)$ the main transition from the upstream to the downstream plasma takes place at the shock ramp and the front of the shock is characterized by the foot region where the magnetic field gradually rises. The upstream magnetic field can be oscillatory and the physics of the foot waves provides rich materials about the waves in plasmas. The foot waves have been interpreted as the whistler mode (Fairfield 1974, Orlowski and Russell 1995, Balikhin et al. 1997a), but their excitation mechanism may not necessarily be unique. For instance, they may result from the gyrating ions or the ring-like ion distributions (Wong and Goldstein 1988, Hellinger et al. 1996), they may be generated in the shock and propagate to the upstream region (Orlowski and Russell 1995), or they may be generated by macroscopic dynamics of the shock (Tidman and Northrop 1968, Krasnosel'skikh 1985, Balikhin et al. 1997a). A variety of instabilities have been suggested at the shock foot and the ramp: ion-ion streaming instability; modified twostream instability; kinetic cross-field streaming instability; lower-hybrid drift instability; ion-acoustic instability; electron-cyclotron drift instability; and whistler instability ( $\mathrm{Wu}$ et al. 1984, Hellinger et al. 1996). The differences of particle dynamics between the electrons and the ions or between the incoming ions and the reflected ions cause the two- 
stream instability in the foot region (Biskamp and Welter 1972, Matsukiyo and Scholer 2003, Scholer and Matsukikyo 2004). The upstream whistler waves which have been detected in the foot region (Fairfield 1974, Balikhin et al. 1997a) are often referred to as the precursor waves as they are a part of the shock structure providing the dissipation (Russell 1988, Burgess 1997, Krasnosel'skikh et al. 2002, Hellinger et al. 2005). Farris et al. (1994) argued that they are phase standing whistler waves. On the other hand, Balikhin et al. (1997a) suggested that the whistler waves escape into the upstream region, as their group velocities are larger than the solar wind speed.

\subsection{Downstream waves}

The physics of the downstream waves is a more complex subject. There are multiple possible sources of waves in the magnetosheath, and the low frequency magnetic field fluctuations can be of the order of the background field strength, which is in the strong turbulence regime. Embedded in the magnetosheath plasma may be fluctuations arising from intrinsic solar wind turbulence processed through the bow shock. Fluctuations may also come from the foreshock region, where they are generated by the reflected ions. The foreshock waves have phase velocities slower than the solar wind speed and are therefore convected with the solar wind toward the shock front and into the magnetosheath. Further magnetosheath fluctuations may be generated at bow shock itself. The temperature increases perpendicular to the magnetic field in the magnetosheath. The temperature anisotropy provides the free energy for plasma instabilities. In a bi-Maxwellian plasma such an anisotropy can drive two instabilities which generate waves with frequencies below the ion cyclotron frequency.

\subsubsection{Ion cyclotron instability}

The first is the electromagnetic ion cyclotron instability. It dominates when the temperature anisotropy is high (with larger $T_{\perp}$ ) and the plasma $\beta$ is low, and generates transverse electromagnetic ion cyclotron waves at frequencies below the ion gyro-frequency with left-hand polarization (Anderson et al. 1991, Fuselier 1992, Gary 1992, 1993, Gary et al. 1993, Gary and Winske 1993, Gary et al. 1994a,b, Gary and Lee 1994). The instability mechanism is based on the cyclotron resonance which isotropizes ions by resonant pitch angle scattering. Ion cyclotron waves typically have phase velocities close to the Alfvén speed and propagate away from their source region. The instability has a maximum growth rate parallel to the magnetic field, causing waves to propagate in this direction with left-hand polarization. All ion species in the plasma contribute to their respective cyclotron instability. In the case of the magnetosheath, proton and helium cyclotron instabilities are expected. 


\subsubsection{Mirror instability}

The second is the mirror instability. It dominates under the conditions of moderate temperature anisotropy and high $\beta$ plasma. It generates large amplitude fluctuations anticorrelated between the magnetic field strength and the plasma density. The mirror mode is a non-propagating mode in the plasma rest frame. In observations large depressions of the magnetic field which were anti-correlated to the plasma density fluctuations have been observed by a number of spacecraft, which was interpreted as the mirror mode. In general, frequencies above zero (i.e. finite propagation speeds) may arise when additional effects such as density or pressure gradients, non-Maxwellian velocity distribution, or nonlinear effects, are taken into account (Hasegawa 1969, Hasegawa and Chen 1989, Johnson and Cheng 1997, Pokhotelov et al. 2001, Gedalin et al. 2001, 2002, Stasiewicz. 2004a,b, 2005). These structures can act as magnetic bottles, trapping part of the particle distribution. There is a resonant mechanism suggested by Southwood and Kivelson (1993) that a group of resonant particles (with no parallel motion) plays a destructive role in the mode excitation, and that the growth rate is inversely proportional to the number of the resonant particles. The mirror instability isotropizes ions by using the magnetic field to pitch angle scatter. When it grows to nonlinear phase, the mirror mode undergoes a saturation mechanism (Kivelson and Southwood 1996). Since the mirror mode structures can be of large amplitude, introducing excess energy into the spectrum over a finite frequency band, it has been suggested that they could lead to both a direct and an inverse cascade of energy to larger and smaller wave numbers (Treumann et al. 2004).

One may then ask which instability dominates, the proton cyclotron, the helium cyclotron or the mirror instability in the magnetosheath. The growth rate of the proton cyclotron instability is generally larger than the mirror instability, provided that the plasma consists of only electrons and protons. With small amount of helium ions taken into account in the plasma, however, the growth rate of the proton cyclotron instability is significantly reduced because the helium ions absorb the growth without affecting the mirror instability very much (Price et al. 1986).

In observations both the ion cyclotron waves and the mirror modes have been observed. Hubert et al. (1998) found the ion cyclotron waves in the outer magnetosheath and the mirror modes in the inner magnetosheath. Denton et al. (1998) also found both waves in the magnetosheath, while Hill et al. (1995) argued that the mirror modes grow quickly near the shock and dominate in the magnetosheath. The ion cyclotron waves tend to be more often observed in the plasma depletion layer near the magnetopause, where the density decreases but the magnetic field increases (Denton et al. 1995, Farrugia et al. 2004).

\subsection{Turbulence}

In in situ observations it is rare to detect monochromatic or quasi-monochromatic waves. Rather, magnetic field fluctuations seem to be random and irregular, suggesting that they 


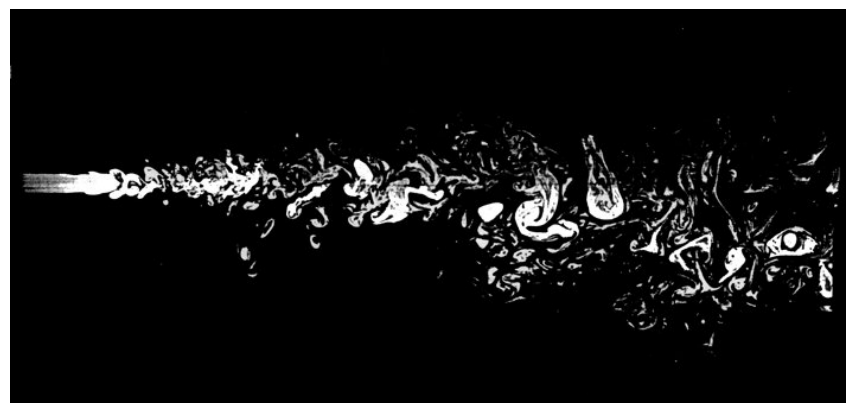

Figure 2.6: Turbulent water jet (Van Dyke 1982). Photograph P. Dimotakis, R. Lye and D. Papantoniou.

are in a turbulent state.

\subsubsection{Hydrodynamic turbulence}

Turbulence is a ubiquitous phenomenon. Whenever fluids are set into motion turbulence tends to develop, as our everyday experience shows us. Waterfalls, clouds, and many other flows in nature exhibit an irregular pattern accompanied by eddies on various scales (Fig. 2.6). In a turbulent flow the structure of the flow is very complex and irregular. The further behavior is unpredictable in the sense that minimal changes would soon lead to a completely different state. Though a direct view of the continuously changing pattern is certainly most eye-catching and fascinating, a pictorial description of these structures is not very suitable for a quantitative analysis. On the other hand, it is just this chaotic behavior which makes turbulence accessible to a theoretical treatment involving statistical methods. A well-known paradigm is the turbulent behavior in our atmosphere. We try to predict the short-term changes, called weather, in a deterministic way for as long as is feasible, as daily experience shows, is not very long, while predictions of the long term behavior, called climate, can be made only on a statistical basis. The probabilistic description is essential in turbulence.

Behind turbulence there lies a restoration of symmetries. The governing equation in hydrodynamics exhibits numerous kinds of symmetries: space reversal, time- and spacetranslation, Galilean transformation, rotations, scaling, and so on. Fluid behavior in hydrodynamics is characterized by a control parameter called the Reynolds number, which is defined as

$$
R=\frac{L V}{\nu}
$$

where $L$ and $V$ denote the characteristic scale and velocity of the flow, respectively, and $\nu$ denotes the kinematic viscosity. As this control parameter is increased, the symmetries 
permitted by the equations (and the boundary conditions) are successively broken. However, at very high Reynolds numbers, there appears a tendency to restore the symmetries in a statistical sense. Such a state is referred to as the fully developed turbulence.

The equation of incompressible fluid motion, the Navier Stokes equation

$$
\frac{\partial \boldsymbol{v}}{\partial t}+\boldsymbol{v} \cdot \nabla \boldsymbol{v}=-\frac{1}{\rho} \nabla p+\nu \nabla^{2} \boldsymbol{v},
$$

is a nonlinear partial differential equation, where $\boldsymbol{v}$ denotes the flow velocity, $t$ time, $\rho$ mass density, $p$ pressure, and $\nu$ viscosity. The Reynolds number represents the ratio of advection (second term on lhs) to diffusion (second term on rhs) magnitude. There are solutions to this equation for simple laminar flows, like honey flowing down a plate, that occur at low Reynolds numbers. At high Reynolds numbers the essence of the problem lies in the nonlinearity. It is not possible to develop a perturbation theory around the linear part of the equation because the theory has no small parameter: quadratic and higherorder terms are the essential ingredients of the problem. Thus perturbation expansions are strongly divergent.

Our "standard model" comes from Kolmogorov who in 1941 postulated that there is a cascade of turbulence energy from largest eddies to smallest eddies, until finally the energy is dissipated by the viscosity (Kolmogorov 1941, Frisch 1995). As the cascade proceeds, the successive generation of smaller eddies loses information of the large scale structure of the flow. Thus the anisotropy of the large scales or the energy injected on the large scale fades and the small scale eddies become statistically isotropic. Kolmogorov postulated that the statistics of these isotropic scales would have universal behavior, independent of the way in which the flow was produced. The scales on which this approximately occurs are known as the universal equilibrium subrange. This is further divided into a dissipation subrange and the inertial subrange. The dissipation range is on the very smallest scales where viscosity becomes dominant. In the inertial range Kolmogorov's theory provides a way of statistics of velocity differences across a separation. Defining this difference $\Delta v(r)=v(R+r)-v(r)$ where $v$ denotes the velocity, $R$ a reference point, and $r$ separation from $R$, the statistical average of $\Delta v(r)$ will be only a function of $\langle\epsilon\rangle$ and $r$ itself, where $\langle\epsilon\rangle$ denotes the average rate of energy dissipation (per unit mass) which is the same as the average rate of energy input in the fully developed turbulence, yielding the scaling relation

$$
\left\langle(\Delta v(r))^{n}\right\rangle \sim(\langle\epsilon\rangle r)^{n / 3}
$$

For $n=2$ the variance $\left\langle(\Delta v(r))^{2}\right\rangle$ will increase as $r^{2 / 3}$. In this case the Fourier transform of Eq. 2.4 yields a $-5 / 3$ spectrum

$$
E_{k}=C_{k} \epsilon^{2 / 3} k^{-5 / 3},
$$

where $C_{k}$ is a constant, $E_{k}$ is energy per unit mass at a wave number $k$. The numerical factor $C_{k}$ is called the Kolmogorov constant and is not determined by scaling arguments. 


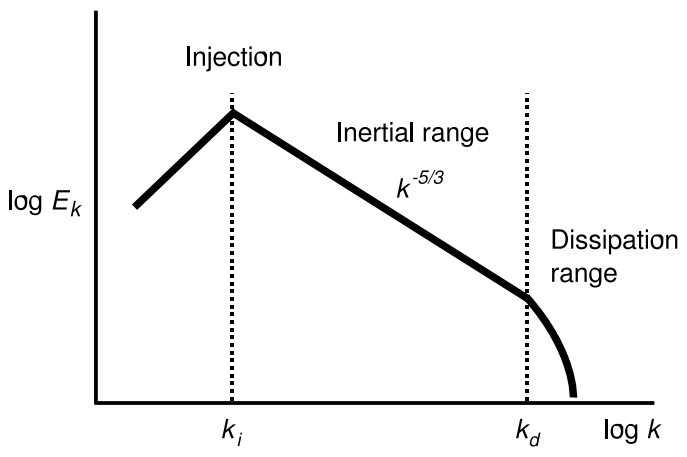

Figure 2.7: A log-log plot of Kolmogorov energy spectrum in the wave number domain showing the energy injection range, the inertial range, and the dissipation range (after Biskamp 2003).

Experimentally $C_{k}$ is invariant with a small statistical scatter, $C_{k}=1.6-1.7$ (Sreenivasan 1995). A typical spectrum is plotted schematically in Fig. 2.7. The energy is injected at the wave number $k_{i}$ and dissipated at $k_{d}$. The inertial range reflects the spectral slope between them.

The spectrum follows also from purely dimensional considerations on assuming that $E_{k}$ depends only on the local value $k$ and the energy-transfer rate $\epsilon$

$$
E_{k} \sim \epsilon^{\alpha} k^{\beta} \text {. }
$$

The exponents $\alpha$ and $\beta$ are determined by matching the dimensions using $\left[E_{k}\right]=L^{3} T^{2}$ and $[\epsilon]=L^{2} T^{-3}$. Various experiments in fluid dynamics confirm Kolmogorov's spectrum nowadays (Frisch 1995, Warhaft 2002).

\subsubsection{Magnetohydrodynamic turbulence}

Kolmogorov's theory is a pillar of modern turbulence theories. There are a variety of turbulence models proposed and each model predicts a unique spectral slope in the inertial range. The $k^{-5 / 3}$ slope reflects ideal, isotropic, incompressible hydrodynamic turbulence (Kolmogorov). The $k^{-3 / 2}$ spectrum reflects ideal, isotropic, incompressible magnetohydrodynamic turbulence, proposed by Kraichnan (1965). The $k^{-2}$ spectrum reflects purely two-dimensional isotropic turbulence, the $k^{-1}$ spectrum shot noise, and the $k^{-3}$ spectrum one-dimensional turbulence.

Turbulence in an electrically conducting fluid is necessarily accompanied by magnetic field fluctuations. It is true that conducting fluids in turbulent motion are rare in our 

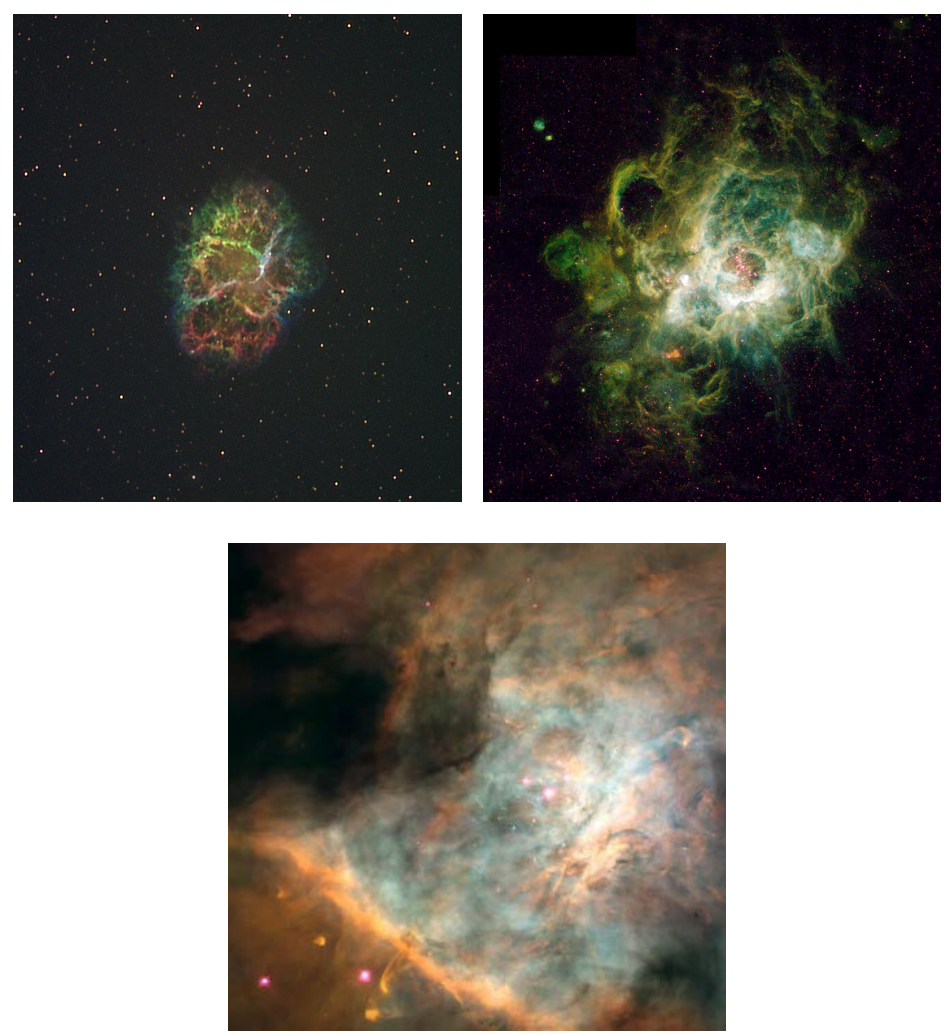

Figure 2.8: Examples of interstellar turbulence: M1 Crab nebula (left top), NGC 604 emission nebula (right top), and trapezium in M42 Orion nebula (bottom).

terrestrial world. In space physics and astrophysics, however, material is mostly ionized and strong turbulence is a widespread phenomenon, for instance in stellar convection zones, solar wind, stellar wind, and in the interstellar medium such as allegoric shapes of nebulae and dark clouds (Fig. 2.8).

Waves, instabilities, and turbulence are complementary to one another. Turbulent flows or magnetic fields may be regarded as superposed waves, while instabilities cause a transition from a smooth flow to turbulent motions. In MHD turbulence the Alfvén effect has been suggested to modify the basic inertial range scaling. The Alfvén effect means that the Alfvén waves propagating in opposite directions along the background magnetic field interact with each other (Iroshnikov 1964, Kraichnan 1965), which may play a crucial role in MHD turbulence, assuming that the cascade dynamics is mainly due 
to scattering of Alfvén waves.

According to this effect, small scale fluctuations are not independent of the macroscopic state but are affected by the large scale magnetic field, and the spectrum becomes

$$
E_{k}=C_{I K}\left(\epsilon V_{A}\right)^{1 / 2} k^{-3 / 2},
$$

which is called the Iroshnikov-Kraichnan spectrum of MHD turbulence. $V_{A}$ is the Alfvén velocity which represents a typical propagation speed of plasma perturbations along the magnetic field and defined as

$$
V_{A}=\frac{B}{\sqrt{\mu_{0} \rho}},
$$

where $B$ denotes the magnetic field strength, $\mu_{0}$ the free space magnetic permeability, and $\rho$ the plasma mass density. The coefficient $C_{I K}$ is expected to be different from $C_{k}$. The spectrum is less steep than the Kolmogorov spectrum. Note that the MHD energy spectrum depends also on the macroscopic quantity $V_{A}$ and therefore cannot be derived by dimensional analysis without additional assumptions. Also, it is interesting to note that the difference between the two spectra is quite small, in spite of the rather different mechanisms.

\subsubsection{Intermittency}

Kolmogorov's and Kraichnan's theories represent fully developed turbulence and they are based on the assumption that the dissipation rate is constant. It has been found, however, that the dissipation rate varies both spatially and temporally within the flow. Because the ultimate fate of the turbulence energy is at the small scales, the dissipation rate is related to the sharp gradients of the velocity that occur there. Thus the dissipation is a function of various combinations of the velocity derivatives and occurs spottily intermittently. In intermittency the self-similar behavior of the energy cascade breaks down and small scale eddies are distributed sparsely, whereas fully developed turbulence exhibits always spacefilling eddies on all scales. This causes the slope of the inertial range spectrum to be propotional to $k^{-2}$, and a probability distribution of velocity (or magnetic field) fluctuation to be non-Gaussian.

\subsubsection{Turbulence in space}

Turbulence in space plasma has been so far most extensively studied in the solar wind using spacecraft-mounted magnetometers and particle detectors, as the solar wind provides an almost ideal laboratory for studying high Reynolds number MHD turbulence. Early analyses by Coleman $(1966,1967,1968)$ indicated that the fluctuations of the solar wind had properties reminiscent of turbulence. In particular, power spectra of the magnetic field or velocity fluctuations often contained an inertial range with a slope of approximately 5/3. More recent work (Matthaeus and Goldstein 1982, Marsch and Tu 1990, Leamon et 
al. 1998) indicates that the spectral slope is more often $-5 / 3$, but it should be noted that the solar wind is neither isotropic, incompressible, nor dissipationless.

In the 1970s and 1980s, impressive advances have been made in the knowledge of turbulent phenomena in the solar wind. Though spacecraft observations were limited by a small latitudinal excursion around the solar equator and, in practice, only a thin slice above and below the equatorial plane was accessible, i.e. a sort of $2 \mathrm{D}$ heliosphere, the observations provided a lot of important results (Tu and Marsch 1995). In the 1990s, with the launch of the Ulysses spacecraft (Wenzel et al. 1992, Marsden et al. 1996), investigations have been extended to the high-latitude regions of the heliosphere, allowing us to characterize and study how turbulence evolves in the polar regions.

In space plasma power spectra of the magnetic field or velocity fluctuations have been solely investigated in the frequency domain, since the spacecraft make one- or at best two-point measurements. To interpret the power spectra in the wave number domain requires the so-called Taylor's hypothesis that all spatial structures are convected by the background flow, and temporal fluctuations reflect simply spatial fluctuations. This assumption, of course, cannot be justified under the existence of waves that have certain propagation speeds. To understand turbulent processes properly, the spectra have to be determined in the wave number domain. 



\section{In-situ wave observations}

\subsection{The Cluster mission}

The complex matter of studying the near Earth space has been restricted to obtaining only one-dimensional views by collecting data simultaneously from one, or at best, two spacecraft. The Cluster mission (Escoubet et al. 2001), however, is capable of multi-point measurements with high time resolution and identical state-of-the-art instrumentation on all of the satellites. After the first proposals in 1982 and beyond the tragic launch failure in 1996, Cluster was rebuilt and launched by the Soyuz rockets from Russian cosmodrome in Baikonur on 16 July and 9 August, 2000. Cluster consists of four spacecraft, forming a tetrahedral constellation with inter-spacecraft separation ranging from $100 \mathrm{~km}$ to $20,000 \mathrm{~km}$. It is designed to study small to large scale structures and fluctuations in three dimensions in regions such as the solar wind, the bow shock, the magnetopause, the polar cusps, the magnetotail, and the auroral zones. It has a polar orbit with a perigee about $4 R_{E}$ and an apogee about $20 R_{E}$. Each Cluster spacecraft carries the same set of eleven instruments: ASPOC (Spacecraft potential control), CIS (Ion composition), EDI (Plasma drift velocity), FGM (Magnetometer), PEACE (Electrons), RAPID (High energy electrons and ions), DWP (Wave processor), EFW (Electric field and waves), STAFF (Magnetic and electric fluctuations), WBD (Electric field and wave forms), and WHISPER (Electron density and waves). Fig. 3.1 and 3.2 display the Cluster spacecraft.

As the magnetic field data from FGM, the ion data from CIS, and the electron data from PEACE are used in this thesis, their respective instrumentation is briefly described below.

\subsection{FGM}

FGM (Fluxgate Magnetometer) is one kind of magnetic field sensor which combines good sensitivity with relative ease of construction. The basic principle is to compare the drivecoil current needed to saturate the core in one direction as opposed to the opposite direction, and the difference is due to the external magnetic field. Let us consider a transformer wound around a high-permeability core. The primary winding of the transformer is excited by high-frequency current. The permeability of the core and the strength of the current are chosen so that the core is driven into saturation on each half cycle of excita- 


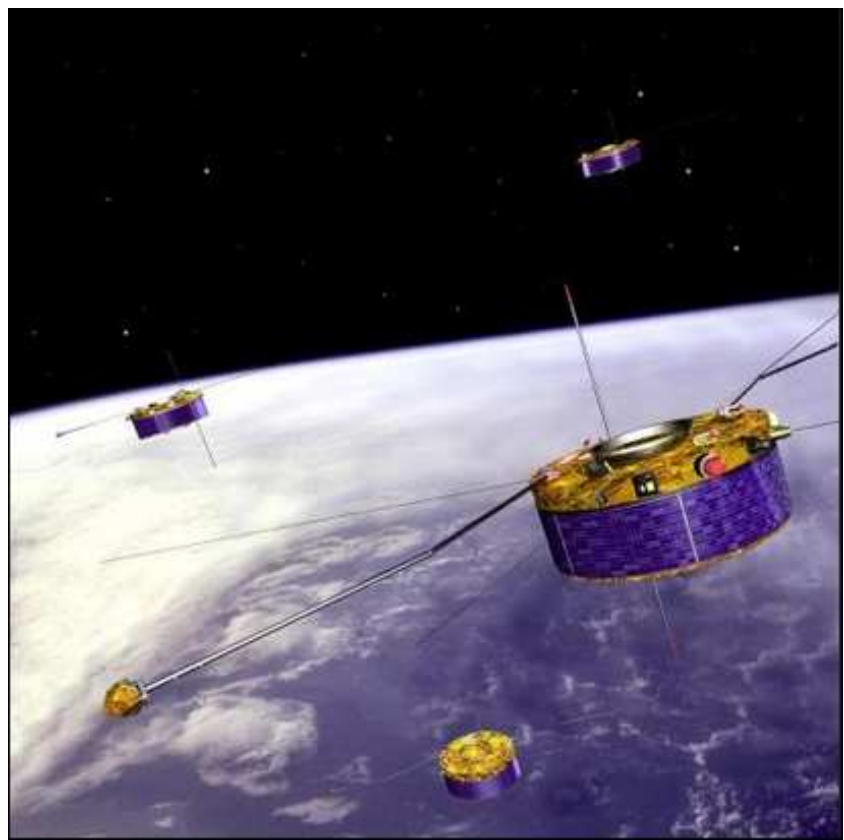

Figure 3.1: Artist's impression of the four Cluster spacecraft (Courtesy of ESA).

tion. The secondary winding detects a time-varying voltage that is related to the input through the hysteresis curve of the core material. For high-permeability materials, this curve is nonlinear and the output signal is highly distorted, containing harmonics of the input signal. The characteristic curve can be written for example in the following form

$$
B(H(t))=3 H(t)-H(t)^{3},
$$

where $H(t)$ denotes the excitation magnetic field at time $t$. The excitation field is oscillatory and can be expressed as

$$
H(t)=H_{e x t}+h \sin \omega t,
$$

where $H_{\text {ext }}$ denotes the external magnetic field, $\omega$ the angular frequency of excitation, and $h$ the amplitude of oscillation. Combining Eq. (3.1) and (3.2), the induced voltage at the secondary coil is then given by

$$
V \sim \frac{d B}{d t}=3 h\left(1-H_{e x t}^{2}-\frac{1}{4} h^{2}\right) \omega \cos \omega t-3 H_{e x t} h^{2} \omega \sin 2 \omega t+\frac{3}{4} h^{3} \omega \cos 3 \omega t .
$$

If there is no external magnetic field along the axis of the transformer (core ring), only odd harmonics of the drive frequency are present in the output. If, however, an external 


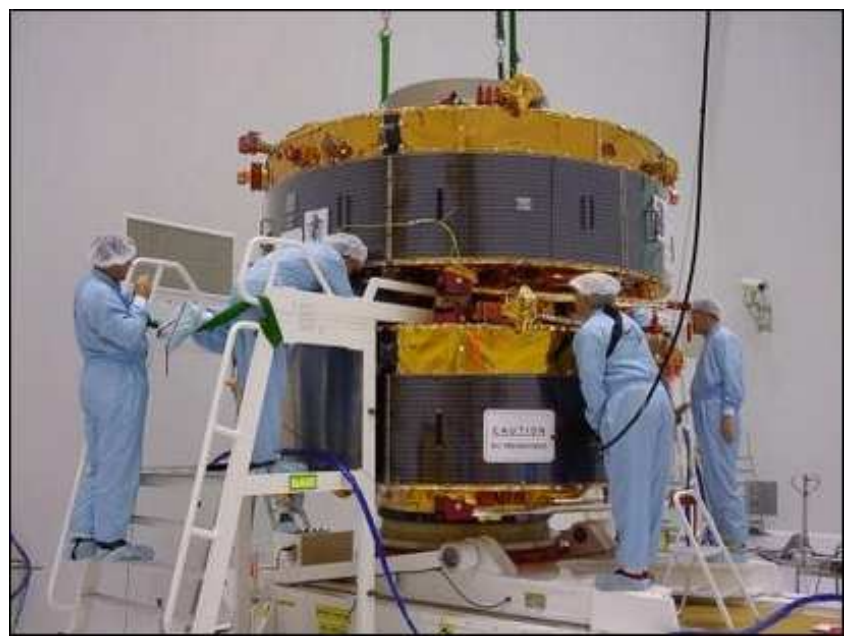

Figure 3.2: Cluster 1 (top) and Cluster 4 (bottom) in a clean room (Courtesy of ESA).

magnetic field is present, even harmonics appear in the output. The amplitude of even harmonics is proportional to the external field and the direction of the field along the transformer axis. In practice, the strength of even harmonics is very weak relative to that of odd harmonics. To amplify and detect these weak signals, the odd harmonics is eliminated by using a suitable coil geometry. In such a case only even harmonics appear in the output. The second harmonics is amplified and detected, giving a voltage proportional to the external field along the transformer axis.

Fig. 3.3 illustrates the electronics needed to measure one component of the magnetic field. A precision oscillator (Oscil) generates a string of pulses at a frequency $2 f_{0}$, where $f_{0}$ is the final drive frequency. This signal is passed to the demodulator circuit as a reference signal, and to a frequency divider $\left(\frac{1}{2}\right)$. The output of the divider $\left(f_{0}\right)$ is passed through a narrow-band filter to the drive amplifier. The string of pulses from the drive amplifier is applied to the primary (Drive) winding of the transformer. A secondary winding around the transformer (Sense) detects the total induced signal and passes it to a preamplifier (Preamp). The output of the preamp passes through a narrow-band filter of frequency $2 f_{0}$ and is further amplified. A strong signal of frequency $2 f_{0}$ is then presented to the synchronous demodulator. This is simply an electronic double-pole, double-throw switch. Each time the input waveform starts to change sign, the switch is activated by the reference signal, making the output signal positive or negative depending on whether the input lags or leads the reference by $180^{\circ}$. The output waveform thus has a frequency of $2 f_{0}$. The output of the demodulator is input to an integrator/amplifier that smooths over many cycles of the rectified waveform, producing a near-dc voltage, with amplitude pro- 


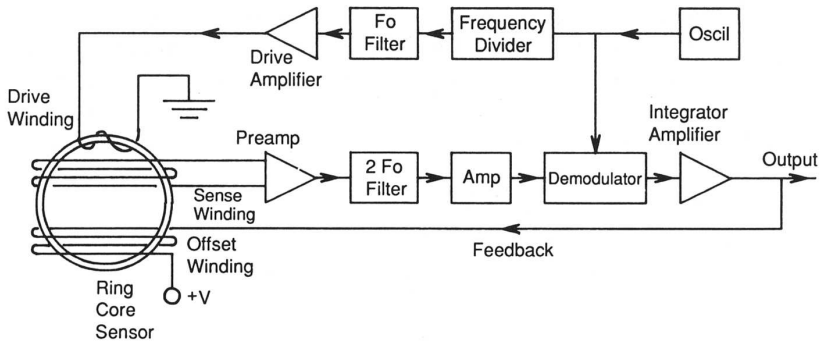

Figure 3.3: Schematic illustration showing the basic components of a (single axis) fluxgate magnetometer (McPherron 1995). The axis of sensitivity lies in the vertical direction, i.e. perpendicular to the sense winding.

portional to that of the second harmonics component output by the sensor, and with sign depending on the phase of the second harmonic relative to the reference signal. These two quantities are, respectively, proportional to the magnitude and direction of the component of the external magnetic field along the axis of the transformer. This low-frequency signal is the magnetometer output. The output voltage is also used to supply a current to an offset winding wrapped around the transformer. The coil constant of this winding and a feedback resistor are chosen so that the current that flows in the offset winding will exactly cancel the magnetic field along the coil axis. Thus, the fluxgate magnetometer serves only as a null detector, making the entire instrument very linear over a large dynamic range. Three components of a vector field are measured by three separate sensors with their ring core axes in mutually orthogonal directions. A fluxgate magnetometer is not an absolute instrument like the proton-precession magnetometer, and so it must be calibrated against standards. Such a calibration requires large non-magnetic test facilities with three-axis calibration coils, proton magnetometers, and optical theodolites.

FGM on each Cluster spacecraft (Balogh et al. 1997, 2001) consists of two tri-axial fluxgate magnetic field sensors on one of the two radial booms of the spacecraft, and an electronics unit on the main equipment platform (Fig. 3.4). The instrument is designed to be highly failure-tolerant through a full redundancy of all its functions. Either of the two magnetometer sensors can be used as the primary sensor for the main data stream from the instrument. In normal operations the outboard sensor located at the end of the $5 \mathrm{~m}$ radial boom is designated as the primary source of the data. The magnetometers can measure the three components of the field in seven ranges from $64 \mathrm{nT}$ (smallest) to $65536 \mathrm{nT}$ (largest). The sampling of vectors from the magnetometer sensor designated as the primary sensor is carried out at the rate of 201.793 vectors/s. This internal sampling rate has been selected to provide an appropriate set of lower rates after filtering for the different telemetry modes and to give the highest frequency response for the short periods of interest. The main operational modes of the FGM instrument provide the sampling rate 


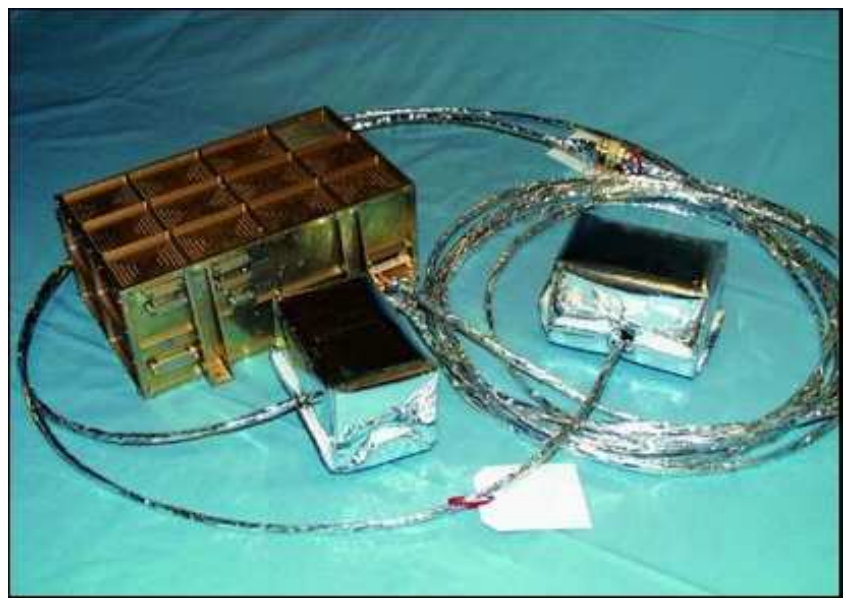

Figure 3.4: Fluxgate Magnetometer for the Cluster spacecraft consisting of Digital Processing Unit (DPU, left), outbound sensor with a long cable (right) and inbound sensor (middle, Courtesy of A. Balogh and E. Lucek).

from 15.52 vectors/s (normal mode) to 67.25 vectors/s (burst mode). The clock signal used for it is derived from a $2^{23} \mathrm{~Hz}(\sim 8 \mathrm{MHz})$ crystal oscillator internal to the instrument. The calibration of FGM was made in Braunschweig.

\subsection{CIS}

The ion data are used for the Doppler shift correction, calculation of the Alfvén velocity, and investigating correlations and coherences with the magnetic field fluctuations. CIS (Cluster Ion Spectrometry) measures both the cold and hot ions of Maxwellian and non-Maxwellian populations (e.g. beams) from various plasma regimes such as the solar wind, the magnetosheath, the magnetosphere, and the ionosphere (Rème et al. 2001). It is designed to achieve following features: (1) A uniform coverage of ions over the entire steradian solid angle with good angular resolution; (2) Separation of the major ion species from the solar wind to the ionosphere $\left(\mathrm{H}^{+}, \mathrm{He}^{++}, \mathrm{He}^{+}\right.$and $\left.\mathrm{O}^{+}\right)$; (3) High sensitivity, large dynamic range, and high time resolution; (4) High angular sampling resolution $\left(5.6^{\circ} \times 5.6^{\circ}\right)$ to detect ion beams and solar wind directions; (5) Wide range of energies up to about $40 \mathrm{keV} / \mathrm{e}$. The CIS instrument consists of two different instruments: HIA (Hot Ion Analyzer) sensor and CODIF (time-of-flight ion COmposition and DIstribution Function) sensor (Fig. 3.5).

HIA measures ion energy per charge by electrostatic deflection in a symmetrical 


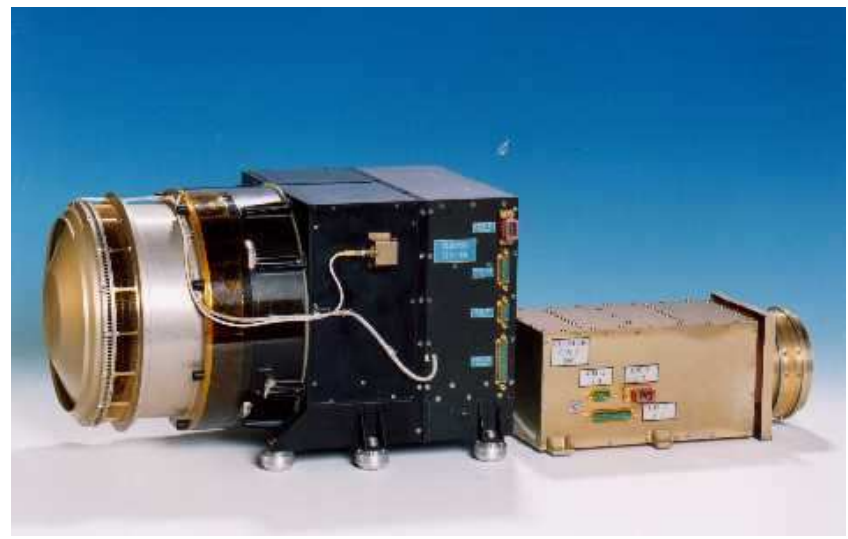

Figure 3.5: CODIF (on the left) and HIA (on the right) ion detectors of the CIS experiment (Courtesy of H. Rème).

quadrispherical analyzer which has a uniform angle-energy response with a fast imaging particle detection system ('top hat' model, Fig. 3.6). This particle imaging is based on microchannel plate (MCP) electron multipliers and position encoding discrete anodes. HIA consists of three concentric spherical elements: an inner hemisphere, an outer hemisphere which contains a circular opening, and a small circular top cap which defines the entrance aperture. In the analyzer an electrostatic potential is applied between the inner and the outer hemisphere, allowing only charged particles with a limited range of energy and initial entrance angle to transmit. The particles are deflected by $90^{\circ}$ (quadrispherical) and detected by MCP. The particle exit position is a measure of the incident polar angle which can be resolved by a suitable position-sensitive detector system. A full $4 \pi$ steradian scan is completed every spin of the spacecraft (about $4 \mathrm{~s}$ ), giving a full 3D distribution of ions in the energy range $5 \mathrm{eV} / \mathrm{e}$ to $32 \mathrm{keV} / \mathrm{e}$.

CODIF is a high sensitivity mass-resolving spectrometer (Fig. 3.7). It measures complete 3D distribution functions of the major ion species $\left(\mathrm{H}^{+}, \mathrm{He}^{++}, \mathrm{He}^{+}\right.$and $\left.\mathrm{O}^{+}\right)$within one spin period of the spacecraft. The sensor primarily covers the energy range between 0.02-38 keV/charge. With an additional Retarding Potential Analyzer (RPA) device in the aperture system of the sensor with pre-acceleration for energies below $25 \mathrm{eV} / \mathrm{e}$. CODIF combines the ion energy per charge with a subsequent velocity measurement (time-offlight analysis) after acceleration to $\leq 15 \mathrm{keV} / \mathrm{e}$. The energy per charge analyzer is a rotationally symmetric toroidal type, which is basically similar to the quadrispheric top hat analyzer used for HIA. RPA allows to extend the energy range below $15 \mathrm{eV} / \mathrm{e}$. The time-of-flight analysis measures the velocity of charged particle through the length of the unit $d$ and the time $\tau$. After passing the electrostatic analyzer the ions are focused onto 


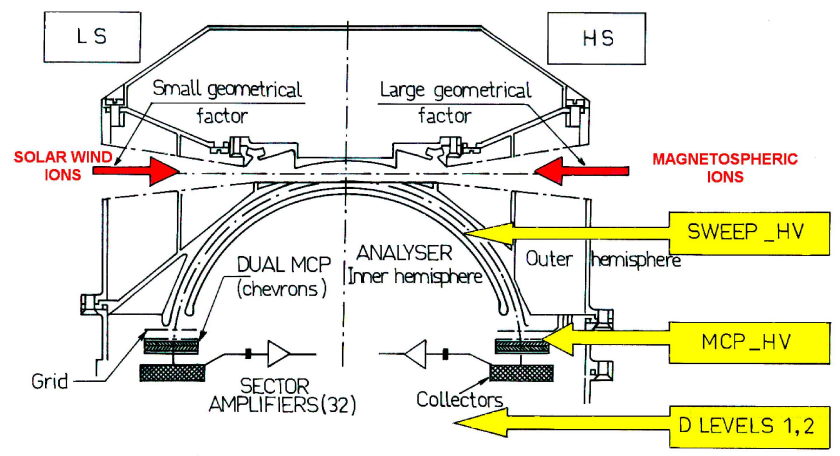

Figure 3.6: Schematic illustration showing the basic components of the HIA sensor (Rème et al. 2001).

a plane close to the entrance foil of time-of-flight (TOF) section, which is held at the potential in order to accelerate the ions. The flight path of the ions is defined by the 3 $\mathrm{cm}$ distance between the carbon foil at the entrance and the surface of the 'stop' MCP. The 'start' signal is provided by secondary electrons, which are emitted from the carbon foil during the passage of the ions. The mass per charge of the ion $M / Q$ is derived as $M / Q=2\left(E / Q+e U_{a c c}\right)(d / \tau)^{-2} \alpha$, where $e U_{a c c}$ is the energy gained by acceleration after the electrostatic analyzer, and $\alpha$ is the effect of energy loss in the thin carbon foil $(\sim 3 \mu \mathrm{g}$ $\mathrm{cm}^{-2}$ ) at the entry of the TOF section.

Both HIA and CODIF are able to measure the velocity distribution function of ions $f(\boldsymbol{v}, \boldsymbol{x}, t)$. One can compute the velocity moments of the distribution function in order to obtain the particle number density, bulk velocity, pressure or temperature, and heatflux (Paschmann et al. 2000). If we simply indicate with $f(\boldsymbol{v})$ the velocity distribution function, we define the quantity $M_{n}$, as moment of order $n$ of the distribution

$$
M_{n}=\int v^{n} f(\boldsymbol{v}) d^{3} v
$$

It follows that the first four velocity moments are the following:

- the number density

$$
n=\int f(\boldsymbol{v}) d^{3} v
$$

- the number flux density vector

$$
n \boldsymbol{V}=\int \boldsymbol{v} f(\boldsymbol{v}) d^{3} v,
$$




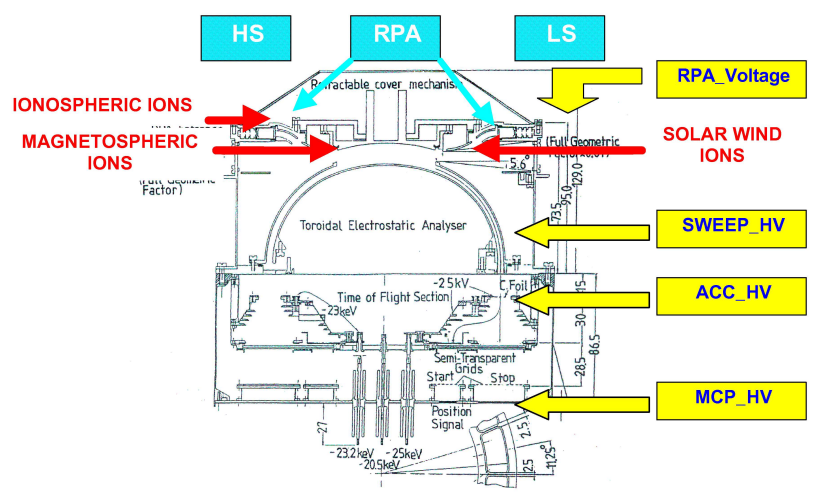

Figure 3.7: Schematic illustration showing the basic components of the CODIF sensor (Rème et al. 2001).

- the momentum flux density tensor

$$
\Pi=m \int \boldsymbol{v} \boldsymbol{v} f(\boldsymbol{v}) d^{3} v,
$$

- the energy flux density vector

$$
\boldsymbol{Q}=\frac{m}{2} \int v^{2} \boldsymbol{v} f(\boldsymbol{v}) d^{3} v
$$

where $m$ denotes the ion mass.

Once the zero-order moment is computed, one can obtain the bulk velocity vector from Eq. (3.6), and compute $\Pi$ and $Q$ in terms of velocity differences with respect to the bulk velocity. Therefore the pressure tensor $\mathcal{P}$ and the heat flux vector $\boldsymbol{q}$ can be obtained from Eq. (3.7) and Eq. (3.8) as:

$$
\mathcal{P}=m \int(\boldsymbol{v}-\boldsymbol{V})(\boldsymbol{v}-\boldsymbol{V}) f(\boldsymbol{v}) d^{3} v
$$

and

$$
\boldsymbol{q}=\frac{m}{2} \int(\boldsymbol{v}-\boldsymbol{V}) \cdot(\boldsymbol{v}-\boldsymbol{V})(\boldsymbol{v}-\boldsymbol{V}) f(\boldsymbol{v}) d^{3} v .
$$

Moreover, using the relation $\mathcal{P}=n k_{B} \mathcal{T}$ one can extract the temperature tensor from Eq. (3.5) and Eq. (3.9). Finally, the scalar pressure $P$ and temperature $T$ can be obtained from the trace of the respective tensors:

$$
P=\frac{\operatorname{Tr} \mathcal{P}}{3}
$$

and

$$
T=\frac{\operatorname{Tr} \mathcal{T}}{3} .
$$




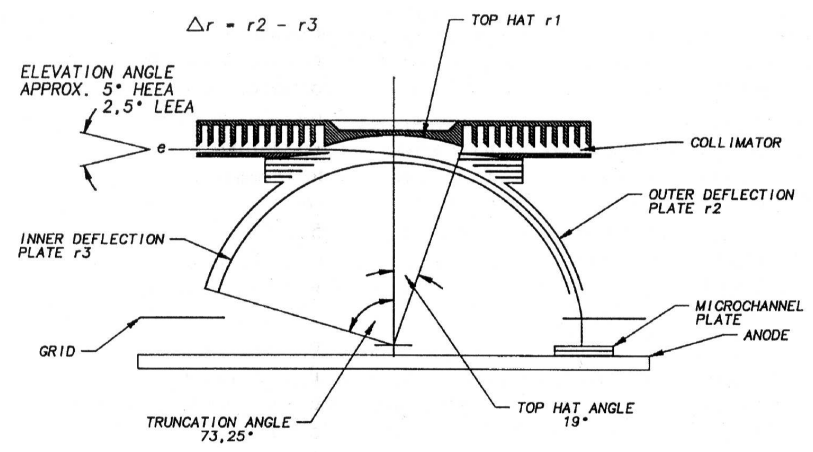

Figure 3.8: Schematic illustration showing the basic components of the PEACE sensor (Johnstone et al. 1997).

\subsection{PEACE}

A PEACE instrument (Plasma Electron And Current Experiment) is designed to measure the electron velocity distribution, covering an energy range of $0.7 \mathrm{eV}$ to about $30 \mathrm{keV}$ and detecting electrons arriving from all possible directions (i.e. $4 \pi$ steradian of solid angle). Each PEACE instrument has two sensor heads, LEEA and HEEA, which are mounted on opposite sides of the spacecraft such that the instantaneous field of view of one is the same as that seen by the other half a spacecraft rotation period later. LEEA and HEEA differ only in geometric factor (HEEA admits more electrons than LEEA in an identical plasma). The measurement principle is the same as CIS-HIA (top hat model) but an opposite sense of electrostatic potential is applied between the inner and the outer hemisphere (Fig. 3.8, 3.9, 3.10). Both sensors sample $4 \pi$ steradians per one spacecraft spin. Note that each sensor covers $180^{\circ}$ in the azimuthal angle seen from the symmetry axis of the sensor, and therefore the three dimensional velocity distribution ( $4 \pi$ steradian coverage) is realized per spacecraft spin.

\subsection{Wave analyses}

Four point measurements of Cluster provide a unique possibility to determine various characteristics of waves. Above all, the investigation of wave vectors serves as a powerful means, from which one can derive not only wavelengths and propagation directions but also phase velocities, dispersion relations, and more. It was not until the launch of Cluster that one obtains wave vectors in three dimension in space plasma observations. The following section introduces how to determine wave properties using both single and multi-point measurements. 


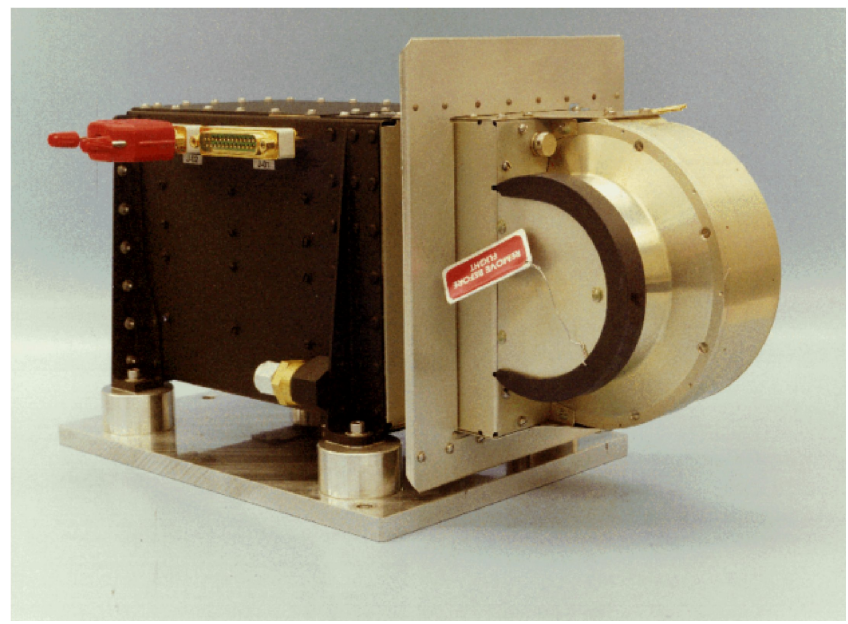

Figure 3.9: PEACE LEEA sensor showing smaller collimator (Courtesy of A. N. Fazakerley).

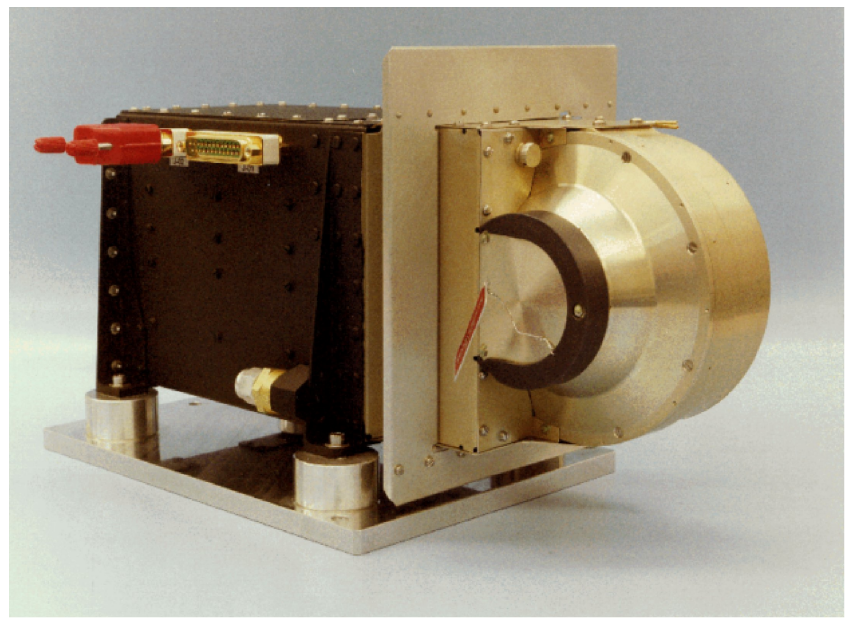

Figure 3.10: PEACE HEEA sensor showing larger collimator (Courtesy of A. N. Fazakerley). 


\subsubsection{Frequency and wave power}

The first step to study waves is to identify frequencies of magnetic field oscillations of interest. It is of course possible to directly count the period of waves in the time series representation, but generally it is difficult to do because waves are not quasi-monochromatic in many cases. Instead, one computes the cross spectral density (CSD) as a function of frequency by Fourier transforming magnetic field data and identifies wave power at various frequencies to see which frequencies are contributing in the fluctuation (Jenkins and Watts 1968, Bendat and Piersol 1980). CSD is calculated as

$$
\mathcal{C}(\omega, T)=\frac{1}{T} \tilde{\boldsymbol{B}}(\omega)^{*} \tilde{\boldsymbol{B}}(\omega),
$$

or using the index notation,

$$
C_{i j}(\omega, T)=\frac{1}{T} \tilde{B}_{i}(\omega)^{*} \tilde{B}_{j}(\omega),
$$

where $\tilde{\boldsymbol{B}}(\omega)$ denotes the Fourier component of the magnetic field fluctuation at the angular frequency $\omega$ for the interval $T$, and the asterisk $*$ denotes the complex conjugate. Subscripts $i$ and $j$ run over the $x, y$, and $z$ component. The diagonal component of CSD, $C_{i i}$, is the wave power with respect to the $i$-th direction. If the $z$-axis is oriented to the mean magnetic field direction, $C_{x x}+C_{y y}$ and $C_{z z}$ yield the wave power in the transversal direction (perpendicular to the mean field direction) and in the compressional direction (parallel to it), respectively. Trace of CSD yields the total wave power

$$
P(\omega)=\operatorname{Tr} \mathcal{C}(\omega) .
$$

To enhance the reliability of output, the interval under investigation is often divided into subintervals, and CSD is computed for each subinterval to average CSD. The more subintervals are used, the more the statistical error is reduced (Jenkins and Watts 1968).

\subsubsection{Ellipticity}

The ellipticity evaluates how the wave field is polarized, right- or left-hand polarized, and circularly or linearly polarized. It is determined by applying the quasi-monochromatic wave theory developed in optics (Fowler et al. 1967, Arthur et al. 1976, Born and Wolf 1980). Provided that the magnetic field fluctuation is expressed in terms of a plane wave as

$$
\begin{aligned}
B_{x}(t) & =a \exp [i(\omega t)] \\
B_{y}(t) & =b \exp [i(\omega t-\pi / 2)] \\
B_{z}(t) & =\text { const, }
\end{aligned}
$$

where $a$ and $b$ are the wave amplitude but can be positive or negative under the condition that $|a| \geq|b|$, CSD at the frequency $\omega$ is calculated as

$$
\mathcal{C}=\left[\begin{array}{ccc}
a^{2} & i a b & 0 \\
-i a b & b^{2} & 0 \\
0 & 0 & 0
\end{array}\right]
$$


The ellipticity $\epsilon$ is defined as the ratio of the minor to the major amplitude

$$
\epsilon=\frac{b}{a}=\tan \phi
$$

where $\phi$ may be determined from CSD as

$$
\sin 2 \phi=\frac{i\left(C_{21}-C_{12}\right)}{\sqrt{\left(C_{11}-C_{22}\right)^{2}+4 C_{21} C_{12}}} .
$$

The value of $\epsilon=+1$ represents the right-hand polarization, 0 for the linear polarization, and -1 for the left-hand polarization.

\subsubsection{Minimum variance analysis}

The divergence-free nature of magnetic fields indicates that field fluctuations are perpendicular to the wave propagation direction (Sonnerup and Scheible 2000). The divergencefree equation is written as

$$
\nabla \cdot \boldsymbol{B}=0 .
$$

When one assumes the magnetic field consisting of a constant background term and a plane wave term as

$$
\boldsymbol{B}=\boldsymbol{B}_{0}+\delta \boldsymbol{B} \exp [i(\omega t-\boldsymbol{k} \cdot \boldsymbol{x})],
$$

substitution of Eq. (3.23) into Eq. (3.22) yields

$$
\boldsymbol{k} \cdot \delta \boldsymbol{B}=0
$$

Thus, the propagation is perpendicular to the direction of the magnetic field fluctuation with the ambiguity of $180^{\circ}$. This method is called the minimum variance analysis, and can be used to determine wave propagation directions using single point magnetic field measurements.

\subsubsection{Correlation and coherence}

Waves exhibit in some cases a correlation or an anti-correlation between the field and the plasma variations. For example, Alfvén waves exhibit correlated magnetic field and plasma velocity fluctuations; and fast magnetosonic waves exhibit correlated magnetic field strength and plasma density fluctuations. The correlation coefficient evaluates how much the fluctuation $x$ is linearly accompanied by the fluctuation $y$, and is defined by

$$
r=\frac{\langle(x-\langle x\rangle)(y-\langle y\rangle)\rangle}{\sigma_{x} \sigma_{y}}=\frac{\langle\delta x \delta y\rangle}{\sigma_{x} \sigma_{y}},
$$

where \langle\rangle denotes the expectation value, and $\sigma_{x}$ and $\sigma_{y}$ are the standard deviation of $x$ and $y$. The value of $r=1$ and $r=-1$ indicate a positive and a negative correlation, respectively. It should be noted that the linear coefficient $r$ is defined to be the square root 
of the fraction of the variance that is explainable by the linear fit and determines part of the variance of the parameter $x$ by the variance of the parameter $y$ when using a linear model $y=\alpha x+\beta$. This implies that $\left(1-r^{2}\right)$ is the fraction of the variance of $y$ that remains unexplained by the linear model. Thus, although the value of $r=0.5$ may sound like a good fit, it means that as much as $75 \%$ of the variance is still unexplained (Reiff 1983).

Coherences and phase angles can also be used to investigate the relation between the variation of $x$ and $y$. The coherence $\gamma$ is defined as

$$
\gamma=\sqrt{\frac{\left|C_{x y}\right|^{2}}{\left|C_{x x}\right|\left|C_{y y}\right|}},
$$

and the phase angle $\phi$ between the two fluctuations is given by

$$
\tan \phi=\frac{\operatorname{Im} C_{x y}}{\operatorname{Re} C_{x y}} .
$$

For example, the set of $(\gamma, \phi)=\left(1,0^{\circ}\right)$ corresponds to the correlation $(r=1)$, and the set $(\gamma, \phi)=\left(1,180^{\circ}\right)$ corresponds to the anti-correlation $(r=-1)$. It is worthwhile to note that even if the correlation coefficient is close to zero, the coherence may not be small in general, for example in such a case that the variation $x$ is retarded or advanced to the variation $y$ by the phase angle $90^{\circ}$ or $270^{\circ}$.

\subsubsection{Wave vector}

The above methods can be applied to any single point measurements. However, the minimum variance analysis provides only the propagation direction with the ambiguity of $180^{\circ}$. Furthermore, it does not derive wavelengths or propagation speeds. Generally speaking, wave frequencies are modulated due to the Doppler shift in a streaming medium, and spacecraft detect frequencies dependent on the flow speed, the spacecraft motion, and wave vectors. In order to correct the Doppler shift, it is necessary to investigate wave vectors $\boldsymbol{k}$. In principle, an infinite number of sensors positioned in space would make it possible to Fourier transform from the spatial domain to the wave number domain, but it is of course unrealistic. It has been found, however, that one can determine the wave vectors from only a few measurement points in space. The method is acknowledged as the wave telescope (or $k$-filtering) technique and this enables one to calculate a wave power as a function of frequency and wave vector. The wave telescope computes a 3 by 3 covariance matrix

$$
\mathcal{P}(\omega, \boldsymbol{k})=\left\langle\boldsymbol{b}(\omega, \boldsymbol{k}) \boldsymbol{b}^{\dagger}(\omega, \boldsymbol{k})\right\rangle
$$

of the magnetic field fluctuation amplitude $\boldsymbol{b}(\omega, \boldsymbol{k})$ at frequency $\omega$ and wave vector $\boldsymbol{k}$, where the dagger $\dagger$ denotes the Hermitian conjugate. Its trace

$$
P(\omega, \boldsymbol{k})=\operatorname{tr} \mathcal{P}(\omega, \boldsymbol{k})
$$


yields the spectral energy density. The task is to find the best estimator for $\mathcal{P}$ using the measured fluctuations $\boldsymbol{b}\left(\omega, \boldsymbol{r}_{s}\right)$ at the positions $\boldsymbol{r}_{s}$ of the spacecraft. The optimal expression is given by Pinçon and Lefeuvre (1991), Motschmann et al. (1995, 1996), Glassmeier et al. (2001) as

$$
\mathcal{P}(\omega, \boldsymbol{k})=\left[\mathcal{H}^{\dagger}(\boldsymbol{k}) \mathcal{M}^{-1}(\omega) \mathcal{H}(\boldsymbol{k})\right]^{-1}
$$

The measured magnetic field fluctuations are contained in the 12 by 12 covariance matrix

$$
\mathcal{M}(\omega)=\left\langle\boldsymbol{B}(\omega) \boldsymbol{B}^{\dagger}(\omega)\right\rangle
$$

with

$$
\boldsymbol{B}(\omega)=\left[\boldsymbol{b}\left(\omega, \boldsymbol{r}_{1}\right), \cdots, \boldsymbol{b}\left(\omega, \boldsymbol{r}_{4}\right)\right]^{T}
$$

The matrix $\mathcal{H}$ is defined as

$$
\mathcal{H}(\boldsymbol{k})=\left[\mathcal{I} e^{i \boldsymbol{k} \cdot r_{1}}, \cdots, \mathcal{I} e^{i \boldsymbol{k} \cdot r_{4}}\right]
$$

where $\mathcal{I}$ denotes the 3 by 3 unit matrix. Deriving expression (3.30) requires to construct a suitable projection procedure which eliminates all signal contributions that do not correspond to a given $\boldsymbol{k}$, that is one needs to consider

$$
\mathcal{P}(\omega, \boldsymbol{k})=\mathcal{W}^{\dagger}(\boldsymbol{k}) \mathcal{M}(\omega) \mathcal{W}(\boldsymbol{k})
$$

with the constraint

$$
\mathcal{W}^{\dagger}(\boldsymbol{k}) \mathcal{H}(\boldsymbol{k})=\mathcal{I}
$$

where $\mathcal{W}(\boldsymbol{k})$ is a weight matrix to be determined. Minimizing the trace of $\mathcal{P}(\omega, \boldsymbol{k})$ under this constraint gives the estimator (3.30) minimizing the noise. Furthermore, the divergence-free nature of the magnetic field may be incorporated into the analysis by demanding $\boldsymbol{k} \cdot \boldsymbol{b}=0$. The effect on the minimized solution (3.30) is to replace $\mathcal{H}(\boldsymbol{k})$ with $\mathcal{H}(\boldsymbol{k}) \mathcal{V}(\boldsymbol{k})$, yielding the optimum result

$$
\mathcal{P}(\omega, \boldsymbol{k})=\left[\mathcal{V}^{\dagger}(\boldsymbol{k}) \mathcal{H}^{\dagger}(\boldsymbol{k}) \mathcal{M}^{-1}(\omega) \mathcal{H}(\boldsymbol{k}) \mathcal{V}(\boldsymbol{k})\right]^{-1}
$$

where

$$
\mathcal{V}(\boldsymbol{k})=\mathcal{I}+\frac{\boldsymbol{k} \boldsymbol{k}}{k^{2}}
$$

The wave telescope assumes that waves represent superposed plane waves and that the the mean field has no large spatial or temporal trend. Determination of wave vectors consists in a search for the highest reliable wave power peaks in $k$-space for a given frequency interval. Two examples of the identified wave vectors are displayed in Fig. 3.11 . 

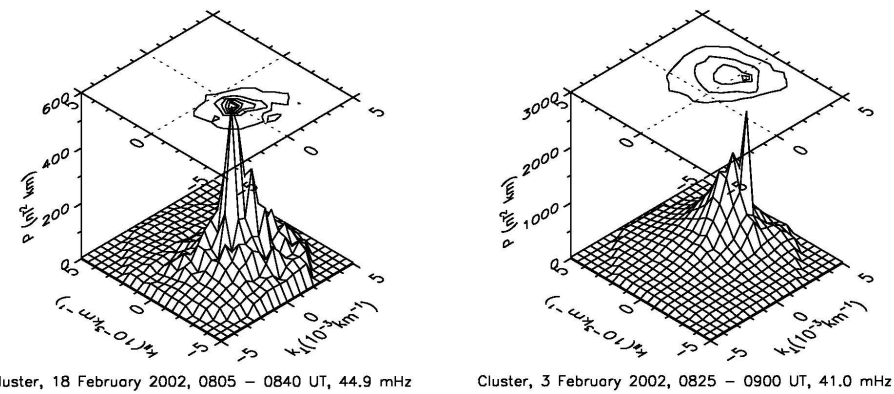

Figure 3.11: Wave power in the $k$-domain derived by the wave telescope technique using Cluster FGM data. The left and right panel show the presence of the anti-parallel and the perpendicular wave vector, respectively (Narita et al. 2004, 2006a).

\subsubsection{Frequency and phase velocity in the plasma rest frame}

Once the wave vector is determined, one can correct the Doppler shift and calculate the frequency in the plasma rest frame using the following relation

$$
\omega_{\text {rest }}=\omega_{s c}-\boldsymbol{k} \cdot \boldsymbol{V}_{\text {flow }},
$$

where $\omega_{\text {rest }}$ and $\omega_{s c}$ denote the angular frequency in the plasma rest frame and in the spacecraft frame, respectively, $\boldsymbol{k}$ the wave vector, and $\boldsymbol{V}_{\text {flow }}$ the plasma flow velocity vector. The spacecraft motion is neglected in this thesis, for it is of the order of a few $\mathrm{km} / \mathrm{s}$, whereas the flow speed in the solar wind and in the magnetosheath is about 400 $\mathrm{km} / \mathrm{s}$ and $100-200 \mathrm{~km} / \mathrm{s}$, respectively. The phase velocity in the plasma rest frame can be further derived as

$$
\boldsymbol{V}_{p h}=\frac{\omega}{|k|^{2}} \boldsymbol{k} .
$$

\subsubsection{Dispersion relation and propagation pattern}

The wave telescope technique provides not only a method to determine the wave vectors but also a variety of analysis tools to identify wave modes and their propagation patterns. The relation between frequencies and wave numbers is called the dispersion relation. From a theoretical point of view, deriving dispersion relations is one of major tasks, since it is uniquely characterized by the distinctive wave mode. Therefore the wave mode can be unambiguously identified if the dispersion relation is properly derived from observations. The dispersion relation can be determined by applying the wave telescope technique and identifying wave vectors at various frequencies.

Another application of the wave telescope is the wave propagation pattern in the medium. When one investigates many examples of wave events in various locations, 
one can determine a distribution of phase velocity vectors or wave vectors in the spatial coordinate, which is a propagation pattern on the statistical basis. 


\section{Dispersion analysis of upstream waves}

\subsection{Introduction}

In this chapter we use the wave telescope technique extensively and aim to derive dispersion relations for the upstream (foreshock) waves. Furthermore, we combine the dispersion curves with polarization properties derived from the minimum variance analysis.

The dispersion analysis was initiated by the ISEE double spacecraft mission. Hoppe et al. (1981) and Hoppe and Russell (1983) estimated wave vectors for the quasi-monochromatic wave packets in the foreshock region, investigating phase differences of the magnetic field fluctuations at the two spacecraft. Also using the magnetic field data obtained by the AMPTE double spacecraft in the same region, Dudok de Wit et al. (1995) and Balikhin et al. (1997a,b) combined the minimum variance analysis with the projected wave numbers and derived approximate dispersion relations.

The data used in the dispersion analysis in this chapter are the magnetic field data from Cluster FGM. The ion data from CIS-HIA are also used to obtain the mean plasma flow velocity $V_{s w}$, the mean mass density $\rho$, and the ion velocity distribution. We have chosen the time interval on February 20, 2002, 1700-1730 UT for the present case. Measurements of spacecraft Cluster 3 are shown in Fig. 4.1. The mean magnetic field is $\boldsymbol{B}_{0}=(8.4,-3.7,5.3) \mathrm{nT}$ in the GSE coordinate system.

\subsection{Dispersion analysis}

The wave telescope determines both wave number vectors and their associated wave power (Pinçon and Lefeuvre 1991, Motschmann et al. 1995, 1996, Glassmeier et al. 2001). We apply the wave telescope at various frequencies up to $11 \mathrm{~Hz}$, which is the Nyquist frequency of the magnetic field data used and derive experimentally dispersion relations in three components of wave number vector. Figure 4.2 exhibits the dispersion relations obtained in the spacecraft $(\mathrm{s} / \mathrm{c})$ frame of reference. The angular frequency $\omega$ is normalized to the proton cyclotron frequency $\Omega_{c p}=1.1 \mathrm{rad} \mathrm{Hz}$ and the wave number vector $\boldsymbol{k}$ is normalized to the ion inertial wave number $\left(V_{A} / \Omega_{c p}\right)^{-1}=10.3 \times 10^{-3} \mathrm{~km}^{-1}$, where $V_{A}$ stands for the Alfvén velocity $B / \sqrt{\mu_{0} \rho}=108 \mathrm{~km} / \mathrm{s}$. For the $k_{x}$ component, a 


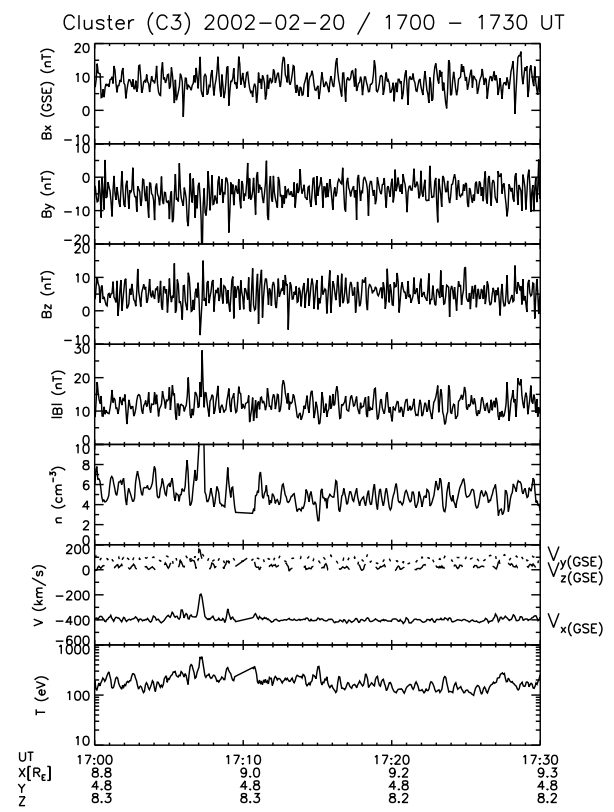

Figure 4.1: Measurements of low frequency waves in the foreshock recorded by Cluster 3 in the interval on February 20, 2002, 1700-1730 UT. Magnetic field, plasma density, velocities, and temperature are displayed in the GSE coordinate system. The CIS data are averaged on 12 seconds (Narita et al. 2003).

linear dispersion branch with negative phase velocity is detected as well as another minor branch at lower frequencies below $1 \mathrm{~Hz}$. For the $k_{y}$ component almost zero wave numbers are detected. For the $k_{z}$ component a linear dispersion branch at lower frequencies below $2 \mathrm{~Hz}$ is smoothly connected to a curved branch at higher frequencies.

In the $\mathrm{s} / \mathrm{c}$ frame, the mean phase velocity is estimated as $\boldsymbol{V}_{p h(s c)}=\omega_{s c} \mathbf{k} /|k|^{2}=$ $(-257,0,-58) \mathrm{km} / \mathrm{s}$. With the background flow velocity $\boldsymbol{V}_{s w}=(-402,83,-17) \mathrm{km} / \mathrm{s}$, one obtains the mean phase velocity in the plasma rest frame $\boldsymbol{V}_{p h(r e)}=\boldsymbol{V}_{p h(s c)}-\boldsymbol{V}_{s w}=$ $(145,-83,-75) \mathrm{km} / \mathrm{s}$. The minus sign in front of $\boldsymbol{V}_{s w}$ compensates the background flow velocity, and $V_{p h(r e), x}$ has an opposite sign with respect to $V_{p h(s c), x}$. Therefore, $\left|\boldsymbol{V}_{s w}\right|$ is larger than $\left|\boldsymbol{V}_{p h(r e)}\right|$ and the spacecraft observe waves propagating in the same direction as the background flow (anomalous Doppler shift). The Doppler shift is corrected at various frequencies using the relation $\omega_{\text {rest }}=\omega_{s c}-\boldsymbol{k} \cdot \boldsymbol{V}_{s w}$, where $\omega_{\text {rest }}, \omega_{s c}$, and $\boldsymbol{k}$ are the angular frequency in the plasma rest frame and in the s/c frame, and the wave number vector, respectively. Without loss of generality we change signs of $\omega$ and $k$ as $\omega \rightarrow-\omega$ and 


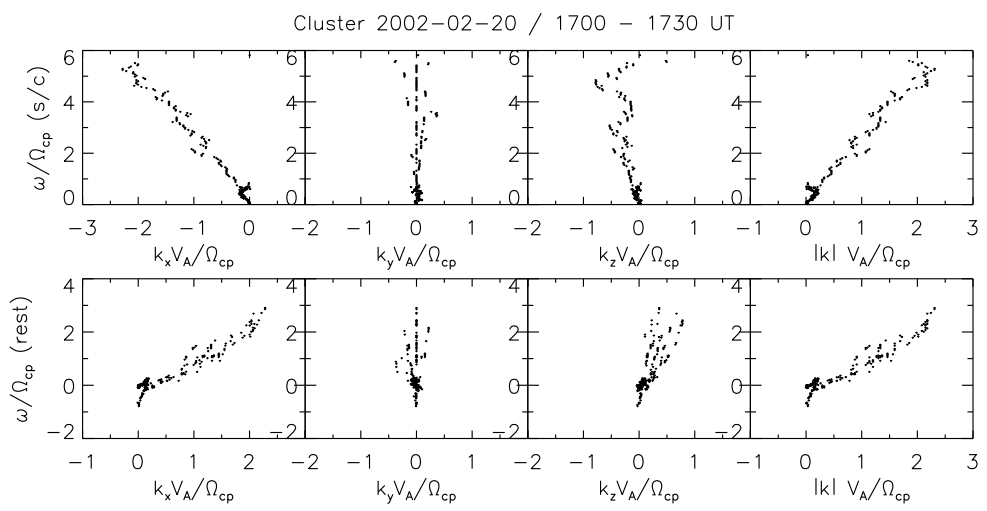

Figure 4.2: Dispersion relations from the wave telescope using Cluster data. Angular frequency $\omega$ is normalized to proton cyclotron frequency $\Omega_{c p}=1.1 \mathrm{rad} \mathrm{Hz}$ and wave number $\boldsymbol{k}$ is normalized to $\left(\left(V_{A} / \Omega_{c p}\right)^{-1}=10.3 \times 10^{-3} \mathrm{~km}^{-1}\right.$, where $V_{A}$ is Alfvén velocity $B / \sqrt{\mu_{0} \rho}=108 \mathrm{~km} / \mathrm{s}$. The GSE system is used for the wave number. Panels in top row show the dispersion relations in the $\mathrm{s} / \mathrm{c}$ frame and bottom panels show the one in the plasma rest frame (Narita et al. 2003).

$\boldsymbol{k} \rightarrow-\boldsymbol{k}$, projecting the dispersion curves into the upper right quadrant. This preserves wave phase velocity but changes representation of wave polarization properties such as the sense of rotation of polarization from a right-handed to a left-handed representation. The resulting dispersion relation is also displayed in Fig. 4.2.

After the Doppler shift correction frequencies are diminished. Some of data points in the dispersion relation are located in the negative $\omega$ regime in the rest frame, i.e. waves propagating in both directions. The linear dispersion branch for $k_{x}$ has been changed to a slightly curved branch. The minor deviating branch at lower frequencies for $k_{x}$ has fallen into the negative $\omega$ regime, showing an almost straight line. The $k_{y}$ dispersion remains almost unchanged, for the Doppler shift is small. The curved branch at higher frequencies for $k_{z}$ has been broadened in the rest frame. Although signs of wave numbers have been changed, $k_{x}$ is kept predominant among the three components and the dispersion relation for $|\boldsymbol{k}|$ exhibits the almost same curves as the $k_{x}$ one.

We investigate, furthermore, the sense of rotation (the sign of ellipticity in the polarization plane) about the propagation direction using the minimum variance analysis (Fowler et al. 1967). This allows one to decompose the dispersion relation into different polarization regimes. Fig. 4.3a shows a dispersion relation derived from the wave telescope in the rest frame plotted together with the sense of rotation at various frequencies. Right- and left-handed polarization are represented by diamonds and plus signs, respectively. A small plot embedded in Fig. 4.3a is an enlarged plot of the dispersion 


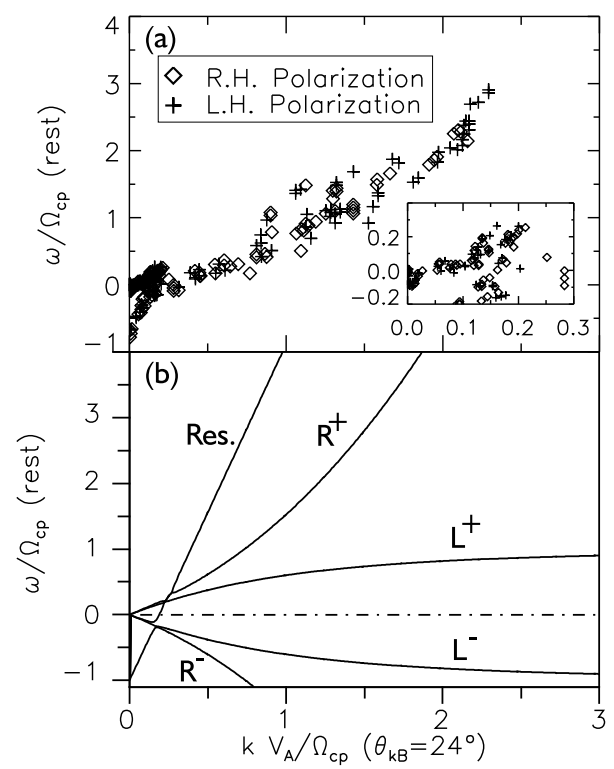

Figure 4.3: Dispersion relations in the plasma rest frame derived from different sources: dispersion from the wave telescope and polarization from the minimum variance analysis for (a) and computation using a multi-fluid plasma model for (b). Wave number $k$ is projected onto a mean propagation direction $\theta_{k B}=24^{\circ}$. Angular frequency $\omega$ and wave number $k$ are normalized as in Fig. 4.2 (Narita et al. 2003).

relation ranged from 0.2 to 0.4 for $\omega_{\text {rest }} / \Omega_{c p}$ and from 0.0 to 0.3 for $k V_{A} / \Omega_{c p}$. Wave number $k$ in Fig. $4.3 \mathrm{a}$ is a projected onto a mean propagation direction $\theta_{k B}=24^{\circ}$, where $\theta_{k B}$ is changed from $156^{\circ}$ to $24^{\circ}$ to agree with the change of sign of $\boldsymbol{k}$ in the rest frame (see above). Both right- and left-handed polarizations are found in the major and minor branch.

To conclude, the direction of the wave propagation is dependent on frames of reference. Waves propagating in the sunward direction in the rest frame are convected in the opposite direction because of the large plasma flow velocity. From the dispersion analysis two different branches are found in the dispersion relation. A major branch shows a linear dispersion in the s/c frame but it is changed into a slightly curved branch in the rest frame. A minor branch intersecting almost perpendicularly to the major branch at lower frequencies in the s/c frame turns out to be an almost linear dispersion branch in the negative $\omega$ in the rest frame. Relevant angles of the wave propagations and the background magnetic field direction are as follows. Propagation angle in the s/c frame and in the rest frame with respect to the Earth-to-Sun direction (GSE-X) are $167^{\circ}$ and $38^{\circ}$, respectively. The IMF 
cone angle and the IMF spiral angle in the XY plane are $38^{\circ}$ and $24^{\circ}$, respectively.

\subsection{Dispersion in a beam plasma}

It is shown that a beam plasma system explains the dispersion relation of the low frequency waves in the foreshock. We assume a beam plasma system composed of two different species: a cold plasma component and a beam with lower density and higher velocity. In a cold plasma treatment there appear four branches in the dispersion relation for transverse low frequency waves for propagation quasi-parallel to the magnetic field, that is right- and left-handed polarization, propagating both forward and backward to the magnetic field. When the ion beam is present a resonant wave emerges due to the ion beam instability. The resonant wave satisfies the cyclotron resonance condition $\omega_{\text {rest }}-k v_{b}+\Omega_{b}=0$, where $v_{b}$ and $\Omega_{b}$ are the velocity and the cyclotron frequency of the beam, respectively.

Assuming the following parameters (justified later) for multi-fluid plasma model gives one a theoretical dispersion relation displayed in Fig. 4.3b: 0.001 (i.e. $0.1 \%$ ) for the ratio of the beam density to the cold plasma density; 5.6 for the Alfvén Mach number of the beam; and quasi-parallel propagation $\left(\theta_{k B}=24^{\circ}\right)$. The wave propagation angle $\theta_{k B}$ has been chosen to be the same as the mean propagation angle from the wave telescope investigation.

In Fig. $4.3 \mathrm{~b}$ there are five branches in the dispersion relation. The linear dispersion branch Res. in Fig. 4.3b stands for a resonant wave of the beam. Other branches originate from the cold plasma: $R^{+}, L^{+}, L^{-}, R^{-}$in Fig. 4.3b, where ' + ' stands for a forward propagation to magnetic field and '-' for a backward propagation, and $R$ for right-handed polarization and $L$ for left-handed polarization.

The dispersion relation Fig. 4.3a is identified as a part of Fig. 4.3b for the following reasons. First, a major curved branch with frequencies up to $3 \mathrm{~Hz}$ in Fig. 4.3a corresponds to the $R^{+}$branch in Fig. $4.3 \mathrm{~b}$, although the curvature of the branch is slightly different. Second, the linear part in the negative $\omega$ in Fig. 4.3a is identified as the linear resonant branch in Fig. 4.3b. When the beam density is smaller enough than the background plasma density, this branch is given by $\omega-k v_{b}+\Omega_{b}=0$. Identification of the linear part of the branch in the experimentally derived dispersion relation allows one to determine $v_{b}$ and $\Omega_{b}$. The beam velocity is estimated as $v_{b}=604 \mathrm{~km} / \mathrm{s}$ in the rest frame, that is 5.6 for the Alfvén Mach number. The beam species are identified as protons, since $\omega_{\text {rest }} / \Omega_{c p} \simeq-1$ at $k=0$ in Fig. $4.3 \mathrm{a}$. These values justify the initial parameters used for the calculation of the dispersion relation in Fig. $4.3 \mathrm{~b}$.

Figure 4.4 is the ion distribution function obtained from the CIS-HIA measurements, exhibiting two gyrating ion populations (reflected ions) around $\left(V_{\perp}, V_{\|}\right)=(-482,79)$ $\mathrm{km} / \mathrm{s}$ and $(579,107) \mathrm{km} / \mathrm{s}$ and solar wind ions around $(232,-339) \mathrm{km} / \mathrm{s}$. The mean difference in $V_{\|}$between the gyrating ions and the solar wind ions is $432 \mathrm{~km} / \mathrm{s}$. This value does not deviate from $v_{b}$ derived from the dispersion analysis. The gyrating ions associ- 


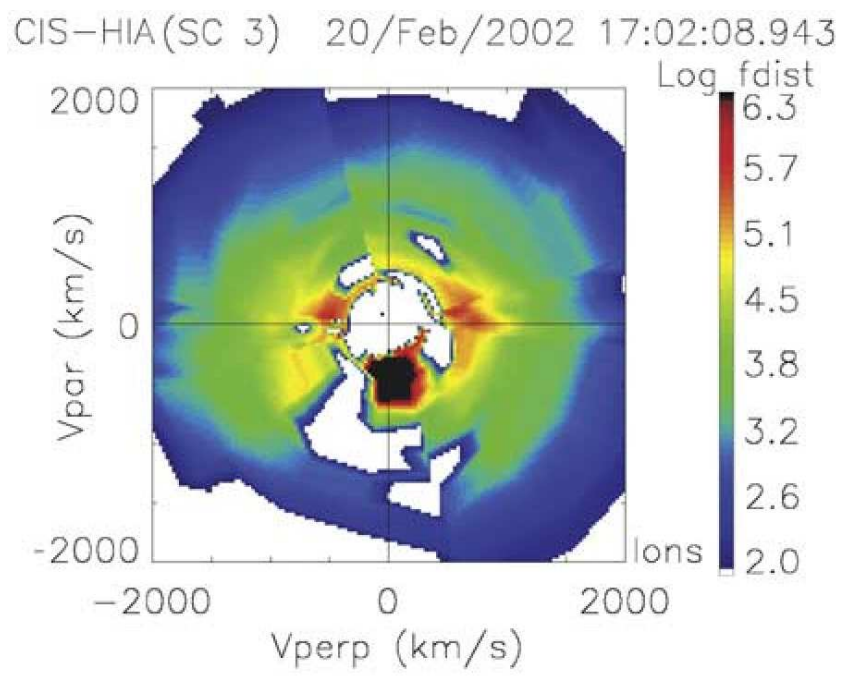

Figure 4.4: Ion distribution function in the velocity space $\left(V_{\perp}\right.$ and $\left.V_{\|}\right)$measured by CISHIA in unit of fdist $\left[\mathrm{s}^{3} / \mathrm{km}^{6}\right] . V_{\perp}$ is along the projection of bulk velocity onto the plane perpendicular to magnetic field (Narita et al. 2003).

ated with the foreshock waves have been reported in Meziane et al. (2001). These ions may be considered as ions which form a beam at first and obtain energy in the perpendicular direction through the instability.

Our scenario for the foreshock waves is that a fraction of the upstream proton population is reflected at the shock forming a beam and subsequently generating low frequency waves by means of the ion beam instability. This is in good agreement with one of the physical processes in the foreshock suggested by a number of observations and theoretical works on the right-hand ion beam instability.

\subsection{Discussion}

The wave telescope has been used extensively to study the dispersion relation of the low frequency waves in the foreshock for quasi-parallel propagation. The dispersion analysis indicates the presence of a backstreaming proton population reflected at the shock and the ion distribution function shows gyrating ions which can be considered as beam protons after the occurrence of the beam instability.

From the dispersion analysis it is also found that the Doppler shift plays an important role in studying wave frequencies and propagation directions. The propagation direction 
is reversed when the Doppler shift exceeds wave frequencies in the rest frame. From a comparison of the dispersion relations it is likely that a beam plasma system explains not only properties of the foreshock waves but also their physical process of wave generation. Although further quantitative investigations, as well as more event studies, are needed to justify our result, backstreaming ions are probably responsible for the generation mechanism of low frequency waves in the foreshock.

We have seen that our result in Fig. 4.3a derived from the wave telescope may be explained by a beam plasma system. Several difficulties, however, need to be solved both qualitatively and quantitatively. First, frequency for the cyclotron resonance and curvature, or group velocity, of the $R^{+}$branch is slightly different. Second, not only right-handed but also left-handed polarization are found in the major branch Fig. 4.3a. In theory, dispersion relations are separated into different polarizations. The sign of ellipticity in the polarization plane obtained from the minimum variance analysis has been adopted to investigate the sense of rotation, but this analysis is subject to noise when determining the polarization plane. Different method will be tested to examine polarizations more, e.g. Stokes parameter method. Third, information on the beam density is not derived from the dispersion relation, because the condition for the cyclotron resonance does not contain the density parameter. The density of the reflected ions from CIS-HIA will be estimated and used to improve the quality of the numerical dispersion relation.

\subsection{Summary}

We have analyzed low frequency wave activity in the near-Earth shock upstream solar wind. Using Cluster as a wave telescope we have investigated in detail wave propagation directions and wave numbers for various frequencies, obtaining, for the first time, three dimensional dispersion relations experimentally. After Doppler shift correction, we find that the dispersion relations are not linear and the waves are propagating in the sunward direction in the plasma rest frame. Comparison of the experimentally derived dispersion relation with that one for a beam plasma system shows good agreement. The results suggest that the waves in the foreshock are generated by the proton population backstreaming from the shock. 



\section{Wave-particle interaction}

\subsection{Introduction}

In observations the existence of whistler waves is often suggested upstream of the shock. In fact they have been observed upstream of most of the planetary bow shocks (Smith et al. 1991, Orlowski et al. 1992, Orlowski and Russell 1995). The $R^{+}$branch in the previous chapter is also a part of the whistler wave dispersion. It has been understood that there is an interplay between the waves and the ions in the upstream region. The waves excited by the ion beam instability can in turn trap a part of ion populations and cause phase-bunched motion of ions. This phenomenon has been detected by the ISEE, WIND, and Cluster spacecraft (Fuselier et al. 1986, Meziane et al. 1997, 2001, Mazelle et al. 2000, Möbius et al. 2001).

This chapter presents an evidence of an interplay between the waves and the electrons. The existence of nongyrotropic electron distributions upstream of the Earth's bow shock is a relatively unstudied phenomenon and may indicate a new physical process in the upstream region. An attempt to identify the wave mode is made and the rotation rate of the nongyrotropic electron distribution is investigated.

\subsection{Wave analysis}

Fig. 5.1 displays the magnetic field fluctuation in the upstream region for the time interval April 29, 2001, 0841-0842 UT. To enhance the quasi-monochromatic feature, the data are band passed through $0.3 \mathrm{~Hz}$ to $1.0 \mathrm{~Hz}$. The associated shock angle from the upstream magnetic field direction determined by the magnetic field coplanarity theorem is about $39^{\circ}$. Fig. 5.2 displays dynamic spectra of the magnetic field fluctuations. Wave power peaks at frequencies about $0.5 \mathrm{~Hz}$.

The results of the wave telescope analysis are shown in Fig. 5.3 (left) plotted in the plasma rest frame. The wave power is computed in the spacecraft frame of reference and transformed into the plasma rest frame using the Doppler shift relation $\omega_{\text {rest }}=$ $\omega_{s c}-\boldsymbol{k} \cdot \boldsymbol{V}_{s w}$, where $\omega_{\text {rest }}$ and $\omega_{s c}$ denote the angular frequency in the plasma rest frame and spacecraft frame, respectively, $\boldsymbol{k}$ the wave vector obtained from the wave telescope analysis, and $\boldsymbol{V}_{s w}$ the local solar wind velocity vector. The wave power is seen to peak in the range $\omega_{\text {rest }} \simeq-2-0 \mathrm{~Hz}$ (corresponding to $0.35-0.45 \mathrm{~Hz}$ in the spacecraft frame) and 
Cluster 4, 29 April 2001, 084100 - 084200 UT

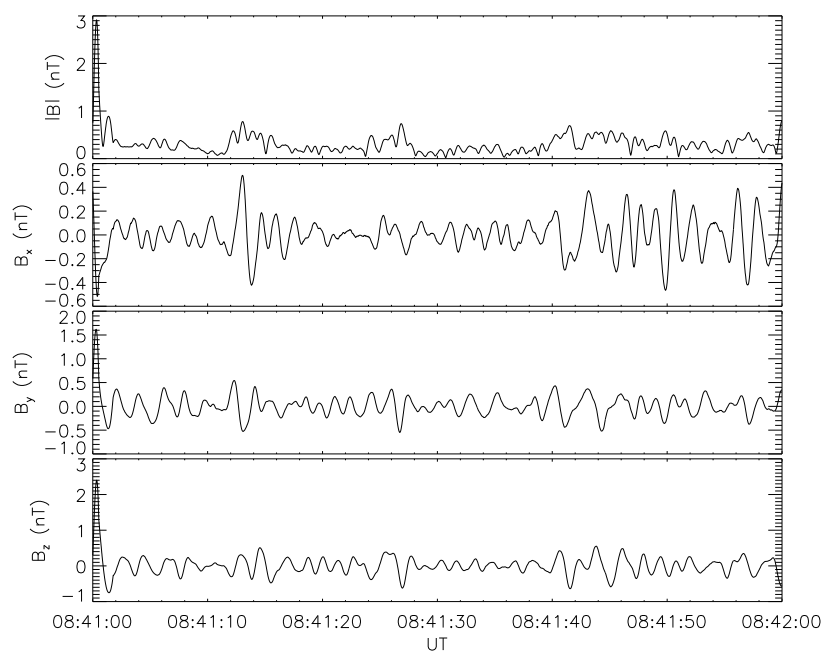

Figure 5.1: Magnetic field input to the wave telescope analysis from Cluster 4. The data have been passed through a $0.3-1.0 \mathrm{~Hz}$ band pass filter and had the background magnetic field subtracted. (Bottom) Dynamic wave power spectrograms for the magnetic field components and total field observed on Cluster 4. Black vertical lines denote the time period of the event. Power has units of $\mathrm{nT}^{2} / \mathrm{Hz}$ (Gurgiolo et al. 2005). The spacecraft is in an outbound orbit and positioned at $[8.7,-7.8,8.8] R_{E}$ in the GSE coordinate system. The spacecraft moves $100 \mathrm{~km}$ outbound within the displayed interval.

$k \simeq 4-6 \times 10^{-3} \mathrm{~km}^{-1}$. The wave is propagating anti-sunward in the spacecraft frame but with the Doppler shift correction it is propagating sunward, as the dominant frequencies are negative in the plasma rest frame.

Fig. 5.3 (right) replots the data in Fig. 5.3 (left) over the restricted frequency range -4 to $4 \mathrm{~Hz}$. In addition the sign of the frequency has been reversed to locate the main dispersion curve in the upper half plane. Changing the sign of the frequency transforms the propagation direction as $\theta_{k B} \rightarrow 180^{\circ}-\theta_{k B}$, which is seen in the lower plot. Overlaid on the upper plot in white is the dispersion curve of the ordinary right-hand whistler wave (cold plasma limit, electrons and ions included, $30^{\circ}$ propagation) computed using the observed local field and plasma conditions: $\mathrm{B}=10.1 \mathrm{nT}$ and $\mathrm{n}=11.8 \mathrm{~cm}^{-3}$. The 


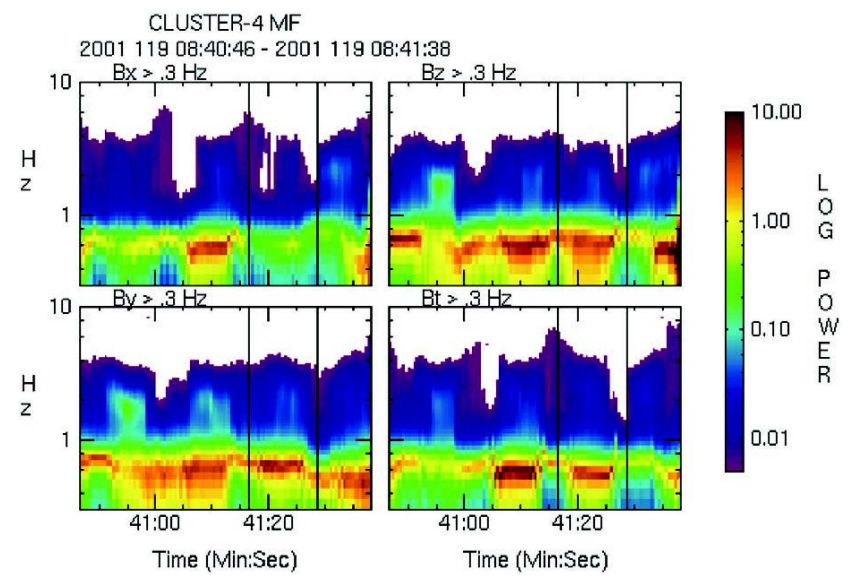

Figure 5.2: Dynamic wave power spectrograms for the magnetic field components and total field observed on Cluster 4. Black vertical lines denote the time period of the event. Power has units of $\mathrm{nT}^{2} / \mathrm{Hz}$ (Gurgiolo et al. 2005).

dispersion relation was determined by the Appleton-Hartree equation

$$
N^{2}=\frac{B \pm \sqrt{B^{2}-4 A C}}{2 A},
$$

which is the equation for the wave refraction index

$$
N=\frac{c k}{\omega},
$$

where $c$ denotes the speed of light, $k$ the wave number, and $\omega$ the wave angular frequency. The coefficients of the equation can be determined by applying the cold plasma model, and they are functions of the background plasma density and magnetic field, the wave frequency, and the propagation direction (Baumjohann and Treumann 1997). The qualitative fit of the theoretical to the derived dispersion curves is a good indication that the waves responsible for the phase trapping are the right-hand whistlers. The derived dispersion relation, however, tends to have larger frequencies and phase speeds than indicated in the theoretical branch shown. This probably implies that not all of the measured electrons and ions contribute to the waves (as assumed in the cold plasma theory). A lower effective density increases the local Alfvén speed. (The whistler wave phase speed at low frequencies approaches the Alfvén speed.) The argument for a lower density is based on the inclusion of the halo electron population in the measured density.

Hodograms of the magnetic field fluctuation in the spacecraft frame of reference show that the wave is left-hand circularly polarized about the magnetic field direction (Fig. 5.4). The waves are right-hand circularly polarized in the plasma rest frame. 

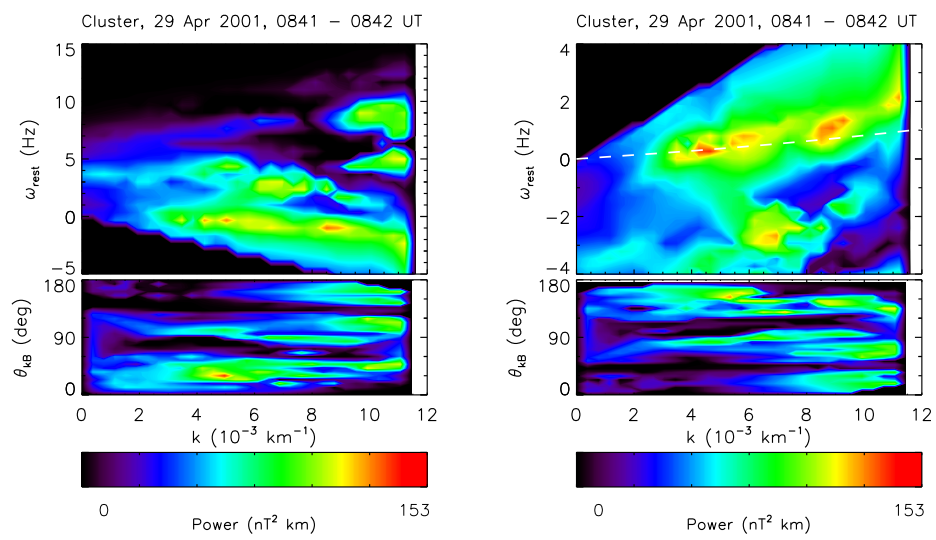

Figure 5.3: (Left) Results of the wave telescope analysis on the 0.3 to $1.0 \mathrm{~Hz}$ band passed magnetic field data. Upper plot is the wave power as a function of the angular frequency and wave number in the plasma rest frame. The lower plot shows the wave propagation angle with respect to the background magnetic field; (Right) Comparison of the ordinary right-hand whistler wave dispersion curve (dashed line) generated using the local magnetic field and plasma parameters with the dispersion pattern arrived through the wave telescope analysis of the local magnetic field data (Gurgiolo et al. 2005).

\subsection{Phase-bunched electrons}

Fig. 5.5 displays a matrix of plots, each of which contains data from the $178.5 \mathrm{eV}$-centered PEACE sweep step taken over one satellite spin. Plots within a column come from one satellite (see label at the top of each column) and within a row from the same spin (as close as can be matched in time between the three satellites). The plots themselves are equidistant cylindrical projections of the velocity space shell containing the measured data. The coordinates are GSE with polar angle plotted along $\mathrm{Y}$ and azimuthal angle plotted along X. A $90^{\circ}$ polar angle points to within $2^{\circ}-6^{\circ}$ of $-\mathrm{Z} \mathrm{GSE}$ and $0^{\circ}$ in azimuth points toward the sun. The measured particle velocity is opposite to these directions so that particles observed at positive polar angles are moving toward the $+\mathrm{Z}$ hemisphere and a particle observed at $0^{\circ}$ azimuth angle is traveling anti-sunward. All plots are in the local plasma rest frame.

The plots show two distinct particle populations: A compact oval of particles at positive polar angles that are high-energy solar wind halo electrons moving anti-sunward and a more spread out population centered near $180^{\circ}$ moving back upstream. The white plus signs within both the halo and return populations are mappings of the head and tail of every magnetic field vector measured during the observations. Their spread indicates 
Cluster 4, 29 April 2001, 084118 - 084122 UT
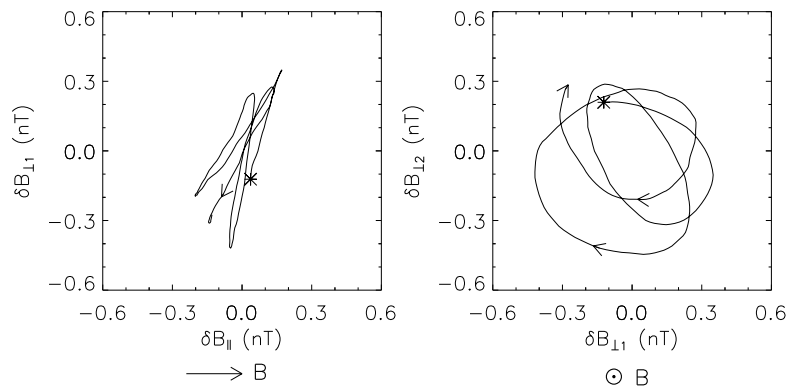

Figure 5.4: Hodogram of the magnetic field at Cluster 4 during the observations of the nongyrotropic electrons. $\delta B_{\perp 1}$ is aligned with the Earth-Sun line and projected onto the plane perpendicular to the magnetic field (Gurgiolo et al. 2005).

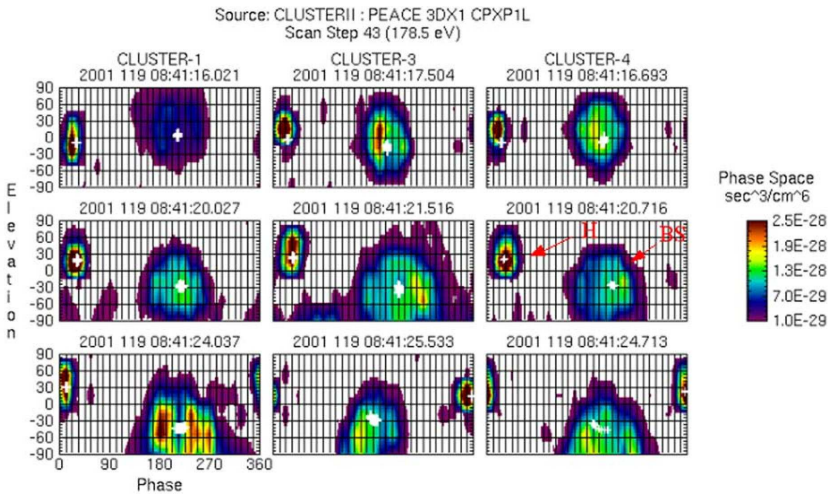

Figure 5.5: Phase-elevation plots of the $178.5 \mathrm{eV}$ channel from Cluster 1, 2, and 3 showing the rotation of the nongyrotropic component of the electron distribution in Cluster 3 and 4. Red arrows in the middle plot in the last column indicate the halo $(\mathrm{H})$ and back-streaming (BS) electron populations (Gurgiolo et al. 2005).

changes in direction of the magnetic field during the measurement. The instrument is looking parallel to the magnetic field when observing the halo electrons. Any apparent offset of the halo electron population from the magnetic field can be attributed to the fact that the instrument measurement bins may not be centered on the magnetic field. Such 
cannot be claimed for the nongyrotropic signatures in the back-streaming electrons which are more than a measurement bin width off the field.

At both Cluster 3 and 4 the return electrons exhibit a clear nongyrotropic signature seen as an intensification at various phases with respect to the magnetic field. Cluster 1 also shows a nongyrotropic signature in the backward streaming electrons but only in the last plot. What is striking about the plots from both Cluster 3 and 4 is the apparent rotation of the nongyrotropic signature in the return beam from spin to spin. As will be shown later, the rotation is real but somewhat deceiving since it cannot be measured directly from the plots.

It is important to understand how the PEACE instrument samples the data in order to understand and interpret what is seen in Fig. 5.5. Fig. 5.6 displays a basic measurement pattern for a single energy step used by the PEACE instrument through one spin of data. There are 384 measurements taken within each spin, one from each of the 12 elevation zones in each of the 32 sectors. The measurements for all of the elevations are taken simultaneously (shaded bar). They occur in a time TM followed by a dead-time TD. The dead-time represents the time expended on the measurements of the other energy steps that make up the full energy sweep. During the measurement cycle the nongyrotropic portion of the distribution is rotating about the local magnetic field. This sets up a beating pattern between the distribution and the measurement cycle. Counts are registered whenever a portion of the distribution overlaps grids in which measurements are being made. The beating of the distribution function with the measurement cycle has two immediate consequences. First, it allows a nongyrotropic distribution to retain a nongyrotropic signature up to the point where the distribution makes one or more rotations within a single measurement time (TM). At that point the distribution will appear in every measurement bin that it overlaps. The data used in this paper has a measurement time of about $0.0039 \mathrm{~s}$ which gives a minimum rotation rate of $256 \mathrm{~Hz}$ before the data would appear as a ring in plots of Fig. 5.5. In actuality, this rate will be slightly smaller due to the thermal extent of the distribution. The second consequence is that when the distribution has a rotation that allows it to move over multiple phase sectors during a measurement time TM, the distribution will generally be oversampled within a spin. The result of this is that the particles may be seen in multiple measurement bins. So while it may be possible to determine if a distribution has a nongyrotropic component, it is often not possible to say anything about its morphology.

An estimation of the rotation period of the nongyrotropic components of the distributions seen in Fig. 5.5 in both Cluster 3 and 4 can be made on the assumption that the rotation period has no time dependency over the $12 \mathrm{~s}$ of data shown. In this case the measured rotation phase should follow the linear relationship

$$
P+360 \cdot N(T)=\Omega \cdot T+P_{0},
$$

where $P$ is the measured rotation phase at time $T, P+360 \cdot N$ is the number of degrees through which the distribution has rotated since $T=0, N$ is an integer, $\Omega$ is the rotation rate in degrees/second, and $P_{0}$ is the distribution phase at $T=0$. The $360 \cdot N$ term 


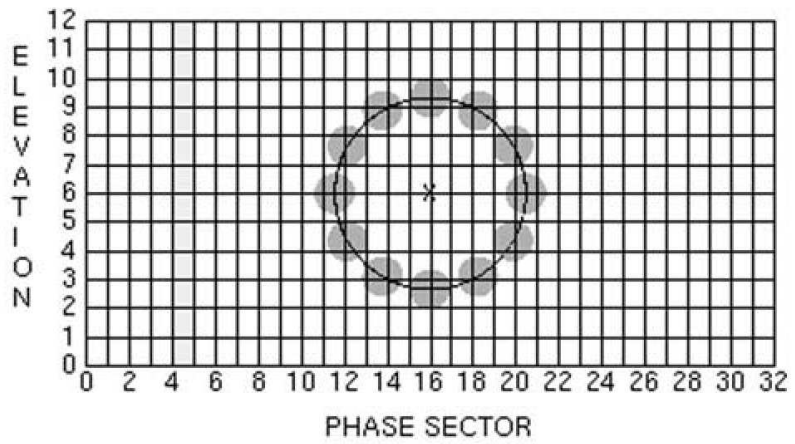

Figure 5.6: The Plasma Electron and Current Experiment (PEACE) measurement matrix for a single energy step. Grids within a column represent the elevation sensors and within a row the azimuthal sectors. Measurements in a column are simultaneous and in a row are sequential. (Gurgiolo et al. 2005)

is required because the distribution may have rotated multiple times between measurements. Note that the term is not a constant in the equation but depends on the time of the measurement.

The approach to solving Eq. (5.3) is to vary $N$ to minimize the standard deviation in the least squares fit. The result of this minimization is shown in Fig. 5.7 for the distributions seen in both Cluster 3 and 4 in Fig. 5.5. In the plot, $x$ represents data taken from main nongyrotropic signature in Cluster 3 and $o$ represents data taken from Cluster 4. $T=0$ is the time of the observation of the nongyrotropic component in the first plot in Cluster 3. All other times are measured relative to this time. Both sets of data show a good fit by the linear relationship in Eq. (5.3) with rotation rates of $\Omega=-180^{\circ} / \mathrm{s}$ (i.e. $f=-0.5 \mathrm{~Hz})$ for Cluster 3 and $\Omega=-175^{\circ} / \mathrm{s}(-0.486 \mathrm{~Hz})$ for Cluster 4 , which are close to the frequencies seen in the waves observed in the magnetic field data (Fig. 5.2). Negative rotation rates correspond to left-hand rotations in the way the problem is set up.

Two additional sets of data are also shown in Fig. 5.7. In the Cluster 3 plots, there is in addition to the primary nongyrotropic signature, a weaker signature that is also rotating. The weaker signature can be seen diagonally opposite to the primary signature and probably results from secondary samplings of the distribution in its gyration. The phase-time relationships for the features were determined identically to that for the primary signature and were added to Fig. 5.7 as number symbols (\#). With the exception of the last observation, they map very well to the established linear rotation-time relationship.

The second set of data added to the plot is represented by the plus symbols. These 


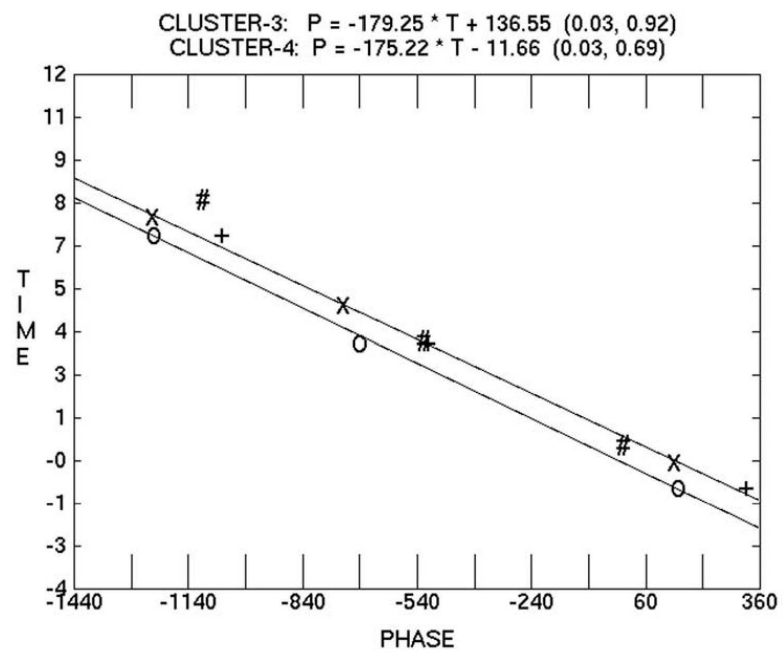

Figure 5.7: The relationship of the nongyrotropic phase angle with time. $T=0$ is the time of the first observation of the nongyrotropic component in Cluster 3 in Fig. 5.5. The fit coefficients for each line are given at the top of the plot with their computed variances in the parenthesis (Gurgiolo et al. 2005).

are the Cluster 4 data (circles) offset in phase by $180^{\circ}$. This shifts the Cluster 4 data to map onto the Cluster 3 data. If the premise that the observed nongyrotropic signatures are phase locked by the locally observed waves is correct, then the separation of the two lines should represent a phase shift in the phases of the waves observed at Cluster 3 and 4.

\subsection{Discussion}

Fig. 5.7 exhibits that the nongyrotropic portion of the distribution is not rotating at the electron Larmor frequency $(\approx 320 \mathrm{~Hz}$ for the local field strength) but is rotating very nearly at the local wave frequency, probably within measurements errors. The largest errors in the computation of the rotation frequency come in the estimation of the rotation phase angle and the assumption that the rotation frequency is a constant over the time period.

Errors in the estimation of the phase of the nongyrotropic distribution are due basically to the finite bin size over which the measurements are taken. The error in phase sector can almost be ignored. The actual measurement occurs within a time interval equal to $1 / 32$ of total sector time. The error introduced by the elevation angle bin is more sig- 
nificant. While PEACE takes measurements in 12 elevation bins, these are often summed for telemetry reasons. At the time of the measurements presented here, PEACE was returning data at only six elevation angles (sums of the adjacent even and odd numbered polar zones). The large bin sizes do not, however, translate into equally large errors in the rotation phase. The narrow range in spin phase restricts the error in the arc tangent when computing the distribution phase and the understanding that the distribution is rotating about a definite circular path centered on the magnetic field (which can generally easily be estimated from the elevation-phase plots) allows an estimation of the elevation angle within a bin. Even an error in the determined phase as large as $\pm 30^{\circ}$ results in only a $\pm 0.05 \mathrm{~Hz}$ error in the rotation frequency. The small error is due to the fact that the distribution has rotated through a much larger angle than is measured from the plots alone.

There are two additional errors that may come into play. The first arises from the assumption that the rotation rate is constant over the three spins during which the measurements are taken. Small changes in the frequency of the peak power shown in Fig. 5.2, especially near the end of the time period, suggest that this probably is not true, which could explain why the last observation of the secondary nongyrotropic feature falls so far off the nominal rotation-time relationship. The overall linearity of the data in Fig. 5.7, however, suggests that the variations in rotation rate are probably not large. The second source of error is a result of the motion of the center of mass of the distribution due to changes in the magnetic field direction during the measurement time frame. This error cannot be larger than the spread in the plus symbols in Fig. 5.5 and in general is much smaller, since the nongyrotropic signature occurs only over a small portion of the time during which the backstreaming electrons are observed.

\subsection{Summary}

The close correlation of the observed rotation frequency of the nongyrotropic electron distribution upstream of the Earth s bow shock with the frequency corresponding to the maximum in the spectral power density in the local waves in the magnetic field suggests a relationship between the two. This is strengthened by the ability to overlay the observations made at Cluster 3 and 4 simply by using the observed phase shift in the magnetic waves between the two satellites.

At this time it is impossible to say whether the magnetic field fluctuations are responsible for the formation of the nongyrotropic signature or if the nongyrotropic electrons are the source of the instability that produces the waves. In either scenario the magnetic waves act as a shepherd wave for the nongyrotropic electrons preventing them from gyrophase mixing as they move upstream of the bow shock.

Using a wave telescope analysis on the magnetic field, the waves are shown to most probably be ordinary right-hand whistler waves. Whistler waves are commonly observed upstream of the Earth s shock. The association of whistler waves and nongyrotropic elec- 
trons may indicate that nongyrotropic electron distribution are more prevalent in the Earth $\mathrm{s}$ foreshock than might be thought. While there are a number of studies which address the formation of the whistlers within the foreshock region both through instability analysis and simulations, none consider the effects of the waves on the background electrons. The results suggest this be undertaken. When these types of studies are completed, not only will we have a better idea of the role of the whistler wave in the formation of nongyrotropic electrons but also what if any role these distributions have in the electron physics taking place upstream of the shock. 


\section{Statistical study of upstream waves}

\subsection{Introduction}

In the previous chapters the wave mode identification through the dispersion analysis is the goal of the study. Investigating wave vectors is a powerful means in wave analyses. It provides phase speeds and their directions, and also brings a possibility to answer the question of how they propagate, namely what their propagation pattern looks like. The propagation pattern in the bow shock environment has not been derived on a statistical basis so far. Yet, the early spacecraft already derived that the propagation sense of the upstream waves is toward upstream (away from the shock) in the plasma rest frame in some case studies (Hoppe et al. 1981, Hoppe and Russell 1983), which is further confirmed in Chapter 4, 5 and also by Eastwood et al. (2002).

This chapter presents a statistical study of the upstream waves. The waves are studied in the plasma rest frame using Cluster data. Magnetic field data of 1 and 4 seconds resolution from FGM are used and the wave telescope is applied to obtain the wave number vectors. Ion density and velocity data from CIS-HIA from Cluster 3 are also used to calculate the Alfvén velocities and the Doppler shifts.

\subsection{Case study}

Fig. 6.1 displays the magnitude of magnetic field, the ion density, and the ion bulk velocity in time domain measured by Cluster 3 from 0400 to 1000 UT on February 18, 2002. The foreshock wave in this event exhibits the clearest example in wave power spectra and typical features which will be drawn in the statistical study. The spacecraft separation is as small as $100 \mathrm{~km}$ and the time series plots of magnetic field display almost the same results among different spacecraft. Cluster observes the dayside northern magnetosphere (0400 - 0500 UT), the magnetosheath (0500 - 0800), and the upstream solar wind region (0800 - 1000) with several shock crossings. The angle between the upstream magnetic field and the shock normal determined from the method of coplanarity theorem is $2.6^{\circ}$ for the Cluster 3 observation. In the upstream region a moderately active fluctuation of the magnetic field is observed between 0800 and 0900 UT with the average summed 


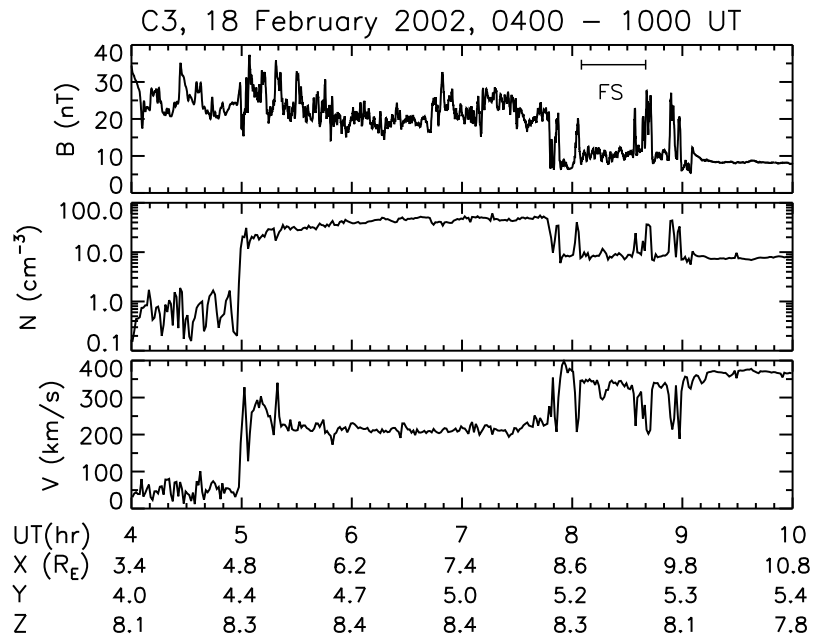

Figure 6.1: Measurements of the magnitude of magnetic field from FGM, ion density and bulk velocity from CIS-HIA made by Cluster 3 from 0400 to 1000 UT on Feb. 18, 2002. 'FS' between 0805 and 0840 UT stands for an interval of the foreshock wave observation (Narita et al. 2004).

component of normalized magnetic field deviation (for 4 seconds data)

$$
\left\langle\sigma^{2}\right\rangle=\left\langle\frac{\left|\Delta B_{x}\right|^{2}+\left|\Delta B_{y}\right|^{2}+\left|\Delta B_{z}\right|^{2}}{|B|^{2}}\right\rangle \sim 0.365,
$$

whereas the fluctuation is quiet after 0900 UT. We take the interval 0805 - 0840 UT for the case study of the foreshock waves. The mean value of interplanetary magnetic field (IMF) is $\boldsymbol{B}=(8.4,-4.1,2.5) \mathrm{nT}$ in GSE and the IMF cone angle (angle between the magnetic field direction and the $x$-axis) is $29.8^{\circ}$. Our analysis method is as follows. First, the geometrical configuration of the foreshock observation is investigated. Then the dominant frequency in the spacecraft frame is identified from the power spectrum and the wave number is identified using the wave telescope. The Doppler shift is corrected to derive the rest frame frequency and the phase velocity. The ellipticity of polarization is also investigated using quasi-monochromatic wave theory.

The foreshock is generally connected to the shock by the magnetic field. We investigate the geometrical configuration for Cluster 3 which provides us with both the FGM and the CIS-HIA data and ask whether the observation is made in the foreshock region or not. We assume that the CIS-HIA data from Cluster 3 are valid for other spacecraft in wave analysis later. For simplicity we adopt an empirical, parabolic bow shock model

$$
X=a_{s}-b_{s}\left(Y^{2}+Z^{2}\right),
$$


where $a_{s}$ is the bow shock standoff distance in unit of $R_{E}$ from the Earth and $b_{s}$ is the flaring parameter in unit of $R_{E}^{-1}$ (Merka et al. 2003). The bow shock is well represented by a paraboloid for GSE- $X$ larger than $-40 R_{E}$ (Cairns et al. 1995). The coordinate system is aberrated so that the direction of $x$-axis is opposite to the solar wind velocity $\boldsymbol{V}_{s w}$ and the $x y$ plane is made by $\boldsymbol{V}_{s w}$ and IMF orientation (we call the $x y$ plane VB plane). The IMF angle $\arctan \left(B_{y} / B_{x}\right)$ in the VB plane is $47.6^{\circ}$. The solar wind dynamic pressure and the fast magnetosonic Mach number calculated from Cluster 3 data are applied to obtain $a_{s}$ and $b_{s}$ using the relations

$$
\begin{gathered}
a_{s}=a_{m p}\left[1+1.1 \frac{(\gamma-1) M^{2}+2}{(\gamma+1)\left(M^{2}-1\right)}\right] \\
b_{s}=0.0223\left(\frac{P_{s w}}{1.8}\right)^{\frac{1}{6}} \quad R_{E}^{-1},
\end{gathered}
$$

where $a_{m p}$ is the magnetopause standoff distance, $\gamma$ is the effective ratio of specific heats and assumed to be 5/3, $M$ is the fast magnetosonic Mach number (Russell 1985), $P_{s w}$ is the solar wind dynamic pressure in unit of nPa. Equation (6.3) and (6.4) were proposed by Farris and Russell (1994) and Cairns et al. (1995), respectively. $a_{m p}$ is calculated for the magnetopause model of Shue et al. (1997). We obtain $a_{s}=14.0 R_{E}$ and $b_{s}=$ $2.25 \times 10^{-2} R_{E}^{-1}$. Now consider the equation

$$
\boldsymbol{r}=\boldsymbol{r}_{0}+D \boldsymbol{e}_{B}
$$

which relates the intersection of the IMF line connected to the spacecraft at the shock $\boldsymbol{r}$ to the spacecraft position $\boldsymbol{r}_{0}=\left(x_{0}, y_{0}, z_{0}\right)$ and the distance between them $D$ (See Fig. 6.2). $\boldsymbol{e}_{B}=\left(e_{x}, e_{y}, e_{z}\right)$ is a unit vector of IMF and it is assumed that there is no change in magnetic field topology. $D$ may take negative values, depending on the direction of IMF and the spacecraft position. Combining equation (6.2) with (6.5), one obtains a quadratic equation for $D$

$$
b_{s}\left(e_{y}^{2}+e_{z}^{2}\right) D^{2}+\left[e_{x}+2 b_{s}\left(y_{0} e_{y}+z_{0} e_{z}\right)\right] D+\left[x_{0}-a_{s}+b_{s}\left(y_{0} e_{y}+z_{0} e_{z}\right)\right]=0 .
$$

Real solutions for $D$ represent the spacecraft located in the foreshock region. We obtain $D=-14.1 R_{E}$. The distance between tangential magnetic field line to the shock and the spacecraft along $\boldsymbol{V}_{s w}$ is $5.2 R_{E}$. Fig. 6.2 displays a sketch of the result in the VB plane translated in $z$ direction to the spacecraft position at $z_{V B}=-26 R_{E}$.

Fig. 6.3 displays the power spectrum of the magnetic field fluctuation derived from the Fast Fourier Transform (FFT) for Cluster 3. A peak in power is identified at frequency $44.9 \mathrm{mHz}$ (period of 22 seconds). Power spectra derived from the other Cluster spacecraft exhibit the same result. The wave number at this frequency is investigated using the wave telescope. An example of the wave power derived from the wave telescope is displayed in Fig. 6.4. A sharp peak is found in the direction almost anti-parallel to the magnetic field. We identify this position as the wave number associated with the given frequency and obtain $\left(k_{\|}, k_{\perp}\right)=\left(-1.4 \times 10^{-3},-0.4 \times 10^{-3}\right) \mathrm{km}^{-1}$ (here $k_{\perp}$ is in the direction of $\boldsymbol{V}_{s w}$ 


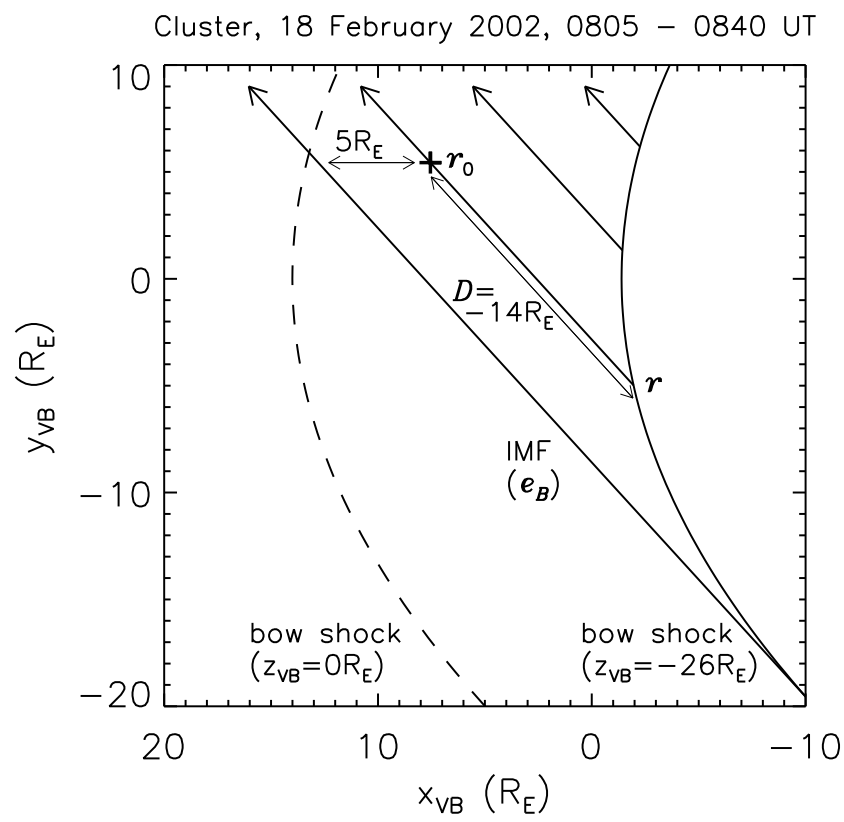

Figure 6.2: Geometrical configuration of the foreshock wave observation between 0805 and 0840 UT on Feb. 18, 2002. The $x$-axis is aberrated to the solar wind direction. The $x y$ plane is made by the solar wind and IMF direction, translated in $z$ direction to the location of Cluster 3 at $z=-26 R_{E}$. Bow shock at $z=0 R_{E}$ projected into $z=-26 R_{E}$ is also present. $\boldsymbol{r}_{0}, \boldsymbol{r}, D$ and $\boldsymbol{e}_{B}$ represent Cluster 3 location, the intersection of IMF line connected to the spacecraft at the bow shock, the distance between $\boldsymbol{r}_{0}$ and $\boldsymbol{r}$, and the unit vector of IMF orientation, respectively (Narita et al. 2004).

projected into the perpendicular plane to the magnetic field) and $\left(k_{x}, k_{y}, k_{z}\right)=\left(-1.1 \times 10^{-3}\right.$, $\left.0.6 \times 10^{-3},-0.7 \times 10^{-3}\right) \mathrm{km}^{-1}$ in GSE. The magnitude of the wave number is $1.47 \times 10^{-3}$ $\mathrm{km}^{-1}$ (wavelength $4284 \mathrm{~km}$ ) and the angle from the magnetic field $\theta_{k B}=163^{\circ}$.

The Doppler shift is corrected and the rest frame frequency is calculated using the relation $\omega_{\text {rest }}=\omega_{s c}-\boldsymbol{k} \cdot \boldsymbol{V}_{s w}$, where $\omega_{s c}=44.9 \times 2 \pi \mathrm{rad} \mathrm{mHz}, \boldsymbol{V}_{s w}=(-317.4,55.8,17.8) \mathrm{km} / \mathrm{s}$, and $\boldsymbol{k}$ as above. We obtain $\omega_{\text {rest }}=-89.2 \mathrm{rad} \mathrm{mHz}$. The physical meaning of the negative frequency becomes clear when discussing the phase velocity. It is defined as $V_{p h}=\omega / k$ or $\boldsymbol{V}_{p h}=\omega \boldsymbol{k} /|k|^{2}$ in vectorial expression. In the spacecraft frame $\boldsymbol{V}_{p h(s c)}=(-144.3$, $83.3,-96.2) \mathrm{km} / \mathrm{s}$ in GSE with the magnitude $192.4 \mathrm{~km} / \mathrm{s}$ and in the plasma rest frame $\boldsymbol{V}_{p h(\text { rest })}=(45.6,-26.3,30.4) \mathrm{km} / \mathrm{s}$ with the magnitude $60.8 \mathrm{~km} / \mathrm{s}$. Thus the direction of 


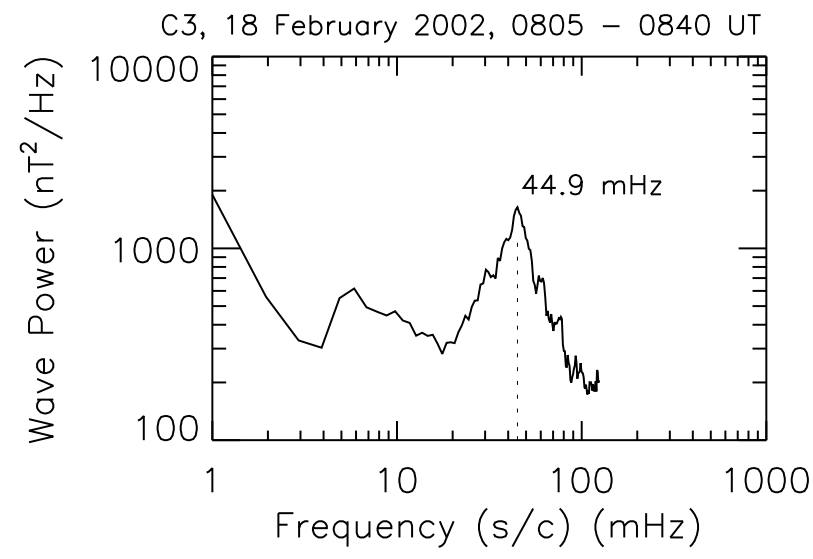

Figure 6.3: Power spectrum in frequency domain in the spacecraft frame for the magnetic field data from Cluster 3 for the same interval as Fig. 6.2 (Narita et al. 2004).

phase velocity is reversed. In the $x$ component, for example, the wave propagates in the anti-sunward direction in the spacecraft frame but in the sunward direction in the plasma rest frame. In other words the wave propagates downstream because the solar wind velocity is larger than the rest frame phase velocity. To avoid the usage of negative frequencies we change the signs of $\omega$ and $k$ without loss of generality. This keeps the phase velocity unchanged but changes the representation of the sense of the polarization from right-hand to left-hand representation and vice versa. We also change the propagation angle $\theta_{k B}$ into $180^{\circ}-\theta_{k B}$ to agree with the change of sign of $\boldsymbol{k}$. As a result, the propagation angle from the sun-to-earth direction is $41^{\circ}$ in the spacecraft frame and $131^{\circ}$ in the plasma rest frame. The normalized frequency is $\omega_{\text {rest }} / \Omega_{c p}=0.096$ and the normalized wave number is $k V_{A} / \Omega_{c p}=0.102$, where $\Omega_{c p}=0.931 \mathrm{rad} \mathrm{Hz}$ and $V_{A}=64.9 \mathrm{~km} / \mathrm{s}$ (Alfvén velocity).

Polarization is also investigated using the principles of optics as applied to quasimonochromatic wave theory. The $i$-th and $j$-th component of the cross spectral density matrix estimator

$$
\hat{C}_{i j}(f)=\lim _{T \rightarrow \infty} \frac{2}{T} E\left[B_{i}^{*}(f, T) B_{j}(f, T)\right]
$$

is calculated (Bendat and Piersol 1980, Born and Wolf 1980). E denotes the operation of expectation (ensemble average) and the indices $i$ and $j$ run over the $x, y$ and $z$ components. $B_{i}(f, T)$ is the finite Fourier transform of magnetic field at frequency $f$ over record length $T$ and the asterisk means the complex conjugate. The polarization plane is then identified by finding eigenvalues and eigenvectors of $\hat{C}_{i j}$, i.e. we search for directions of the principal and second principal variance. The ellipticity $\epsilon$ (ratio of minor to major 


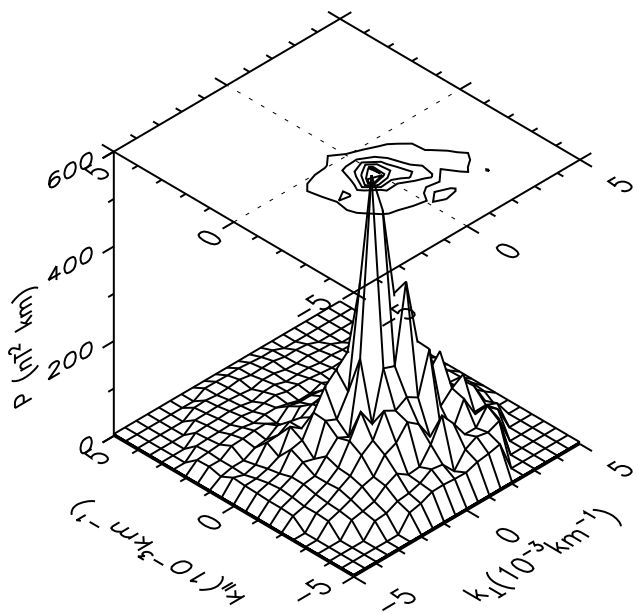

Cluster, 18 February 2002, 0805 - 0840 UT, 44.9 mHz

Figure 6.4: Wave power in $k$-space made by the wave telescope and the magnetic field data from all four Cluster for the same interval as Fig. 6.2. $k_{\perp}$ is in the same direction as ion bulk velocity projected into the perpendicular plane to the magnetic field (Narita et al. 2004).

axis in the polarization plane) is defined by Eq. (3.20) and Eq. (3.21). The ellipticity is compared among different spacecraft and exhibit the result very close to one another in frequency domain, i.e. polarization is coherent for $100 \mathrm{~km}$ distance. We obtain $\epsilon=0.187$ for the given frequency, thus the wave is elliptically right-hand polarized in the spacecraft frame. In the plasma rest frame the sign of $\epsilon$ is changed, resulting from the reversal of propagation direction (Strictly speaking, the ellipticity in this context is an estimator and should be expressed with a different symbol like $\hat{\epsilon}$, but we use simply $\epsilon$ to refer to it). In summary, we have derived the following properties in the plasma rest frame: frequency $\sim 0.1 \times \Omega_{c p}$; wave number $\sim 0.1 \times \Omega_{c p} / V_{A}$ (also the wavelength of the order of $R_{E}$ ); phase velocity $\sim V_{A}$; propagation almost along the magnetic field $\left(\theta_{k B}=17^{\circ}\right)$; and elliptically left-handed polarization.

\subsection{Statistical study}

In the following statistical study we present various distributions of the foreshock wave properties: frequencies, wave numbers, phase velocities, propagation directions, and polarization, applying the analysis method introduced in the previous section. Intervals used 


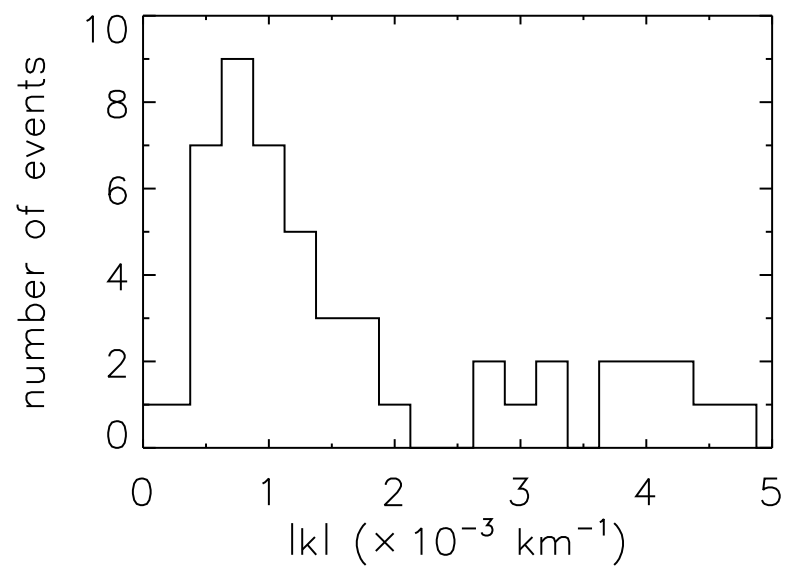

Figure 6.5: Histogram of magnitude of wave number (Narita et al. 2004).

for the statistical study are selected as follows.

(A) The mission phase for $100 \mathrm{~km}$ spacecraft separation (from 3 February 2002 to 17 June 2002) is selected in order to resolve waves into small wavelengths up to 200 $\mathrm{km}$; (B) Bow shock crossings are identified by searching for the following characteristics: (a) An increase in the magnetic field magnitude when moving from the upstream solar wind to the magnetosheath (typically by a factor of 2 to 4); (b) a decrease in the ion bulk speed; (c) an increase in the ion density. The ion bulk speed $\sim 400 \mathrm{~km} / \mathrm{s}$ is also referred to in identifying the upstream region; (C) Moderately active fluctuation of the magnetic field is identified in the upstream region. For this purpose the variance of the fluctuation (defined in Section 2) $0.05 \leq \sigma^{2} \leq 0.40$ is imposed as a criterion; (D) The geometrical configuration is investigated and the intervals in the foreshock region are selected, applying the same method as described in the previous section.

For the selected intervals dominant frequencies are identified up to $0.5 \mathrm{~Hz}$ in the spacecraft frame. 61 distinct waves from 36 events are selected under these criteria. Then wave numbers, rest frame frequencies, and phase velocities are investigated. Ellipticities are investigated as well. Table 6.1 lists all the intervals and frequencies in the spacecraft frame. The histogram of magnitude of wave number is displayed in Fig. 6.5, which can be derived only from multi-point measurements. We find that most of wave numbers have magnitude up to $2 \times 10^{-3} \mathrm{~km}^{-1}$. When normalized, this distribution exhibits a peak around $0.1 \times V_{A} / \Omega_{c p}$ (discussed later).

Fig. 6.6 displays the spatial distribution of phase velocities in the plasma rest frame projected into the $x r$ plane in GSE $\left(r=\sqrt{y^{2}+z^{2}}\right)$. Small circles filled in black are the locations of wave observations and arrows starting from the circles are the phase velocities 


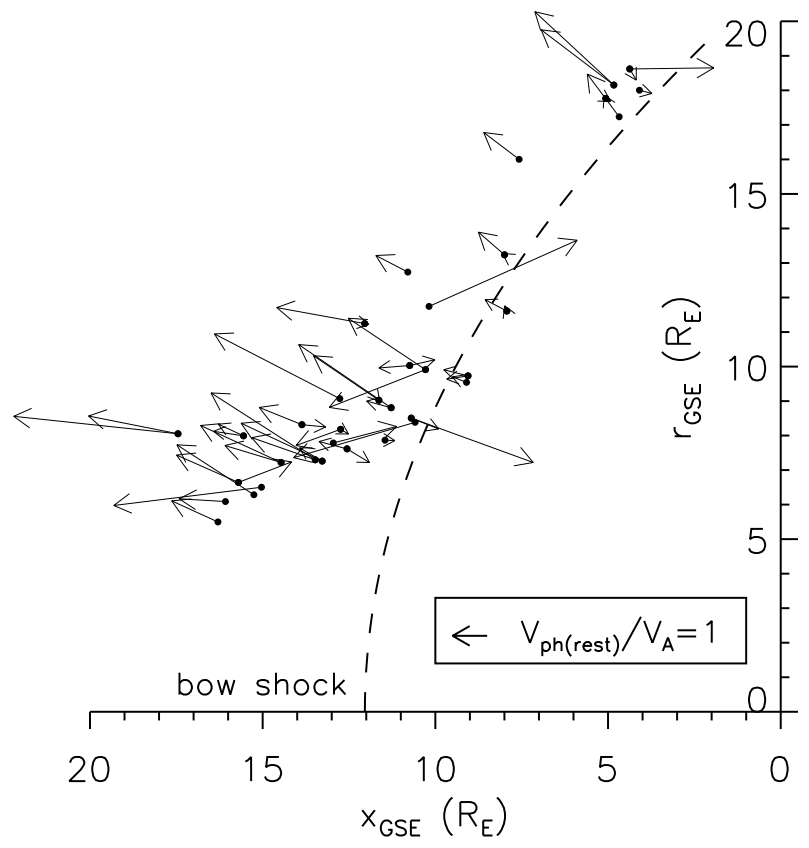

Figure 6.6: Spatial distribution of wave phase velocities in the plasma rest frame (arrows) plotted together with the location of wave observations (filled circles). The phase velocities are projected onto the $x r$ plane in GSE, where $r$ is the radial distance from the $x$-axis, $r=\sqrt{y^{2}+z^{2}}$. The phase velocities are normalized to the local Alfvén velocity. The dashed, curved line represents a bow shock position calculated for a quiet solar wind condition

(Narita et al. 2004).

normalized to the local Alfvén velocity $V_{A}$. The dashed curved line represents a nominal bow shock for quiet solar wind conditions. We find that most of the waves propagate upstream and are more or less aligned with shock normal directions at various positions, near the $x$-axis to near flank region. The phase velocities are of the order of the Alfvén velocity. Some of the waves, however, propagate downstream in the plasma rest frame. Such waves tend to have phase velocities smaller than the Alfvén velocity but a few cases show larger phase velocities near the shock.

The distributions of frequencies, wave numbers, propagation angles, and ellipticities are displayed in Fig. 6.7. Error bars are also present there. For scanning in the $k$-space 
we use a spherical grid with $75 \times 60 \times 60$ points in radial, azimuthal, and polar direction between $k_{\text {min }}=0 \mathrm{~km}^{-1}$ and $k_{\text {max }}=5 \times 10^{-3} \mathrm{~km}^{-1}$ for low-frequency waves up to $100 \mathrm{mHz}$ in the spacecraft frame, and between $0 \mathrm{~km}^{-1}$ and $25 \times 10^{-3} \mathrm{~km}^{-1}$ for the rest of frequencies. Mean $|\Delta \boldsymbol{k}|$ is thus about $6.7 \times 10^{-5} \mathrm{~km}^{-1}$ for low-frequency waves and $3.3 \times 10^{-4} \mathrm{~km}^{-1}$ for high-frequency waves, and $\Delta \theta_{k B}$ is $3^{\circ}$. Errors in the rest frame frequencies represent uncertainty in the Doppler shift, i.e. $\left|\Delta \boldsymbol{k} \cdot \boldsymbol{V}_{s w}\right|+\left|\boldsymbol{k} \cdot \Delta \boldsymbol{V}_{s w}\right|$. We use $\Delta \boldsymbol{k}$ above and $\Delta \boldsymbol{V}_{s w}$ which is calculated from mean absolute deviation of the ion velocity for Cluster 3 CIS-HIA. We do not assign error bars in the ellipticities, since the eigenvectors of spectral density matrix are uniquely determined (without any uncertainty). There are, however, various methods to investigate polarization parameters. Comparison among these methods may give the error estimate in the ellipticities. The left panel is the distribution of normalized frequencies $\omega_{\text {rest }} / \Omega_{c p}$ and magnitudes of normalized wave numbers $k V_{A} / \Omega_{c p}$. The signs of $\omega_{\text {rest }}$ and $k$ are already changed, since most of the frequencies become negative after the Doppler shift correction. A magnified plot around $\omega / \Omega_{c p} \sim 0$ and $k V_{A} / \Omega_{c p} \sim 0.1$ is also embedded in the left panel. The dotted, straight line represents Alfvén waves propagating along the magnetic field with the relation $\omega / k=V_{A}$. Most of the waves are found at $\omega / \Omega_{c p} \sim 0.1$ and $k V_{A} / \Omega_{c p} \sim 0.1$, therefore the major population of the waves propagates at speeds below and around up to the Alfvén velocity. Minor waves are found up to $\omega / \Omega_{c p} \sim 5$ and $k V_{A} / \Omega_{c p} \sim 1.2$. These waves are scattered in the $\omega-k$ distribution. Some of them follow $\omega / k=V_{A}$ and others deviate from it. The middle panel in Fig. 6.7 is the distribution of propagation angles $\theta_{k B}$ versus frequencies. The first major population is found at $\theta_{k B}>160^{\circ}$ and the second major population is found at $\theta_{k B}<30^{\circ}$. These two populations have small frequencies which correspond to the waves of $\omega / \Omega_{c p} \sim 0.1$ and $k V_{A} / \Omega_{c p} \sim 0.1$ in the left panel. Minor waves with relatively large frequencies and wave numbers have propagation angles perpendicular to the magnetic field rather than parallel/anti-parallel. The right panel in Fig. 6.7 is the distribution of ellipticities. The ellipticities are located between -0.5 and 0.2 . Major waves have distribution centered slightly on the left-hand polarization side. Minor waves with larger magnitude of frequencies tend to be left-handed for positive frequencies (upstream propagation) and right-handed for negative frequencies (downstream propagation).

In summary, most of the waves have frequencies $\sim 0.1 \times \Omega_{c p}$ and wave numbers $\sim 0.1 \times \Omega_{c p} / V_{A}$. They propagate upstream almost parallel/anti-parallel to the magnetic field, at phase velocities close to $V_{A}$ with left-hand polarization. We interpret that the dominant waves represent Alfvén waves because of good agreement in the phase velocity at low frequencies. Minor waves are also present, having propagation angles roughly perpendicular to the magnetic field at various phase velocities. Some minor waves agree with the dispersion relation of the Alfvén wave $\left(\omega / k=V_{A}\right)$ at larger frequencies and wave numbers. We also examined if there is a possible relationship between frequencies or wave numbers and distances from the shock but they were relatively uniformly distributed and did not exhibit any clear signatures or organizations. 

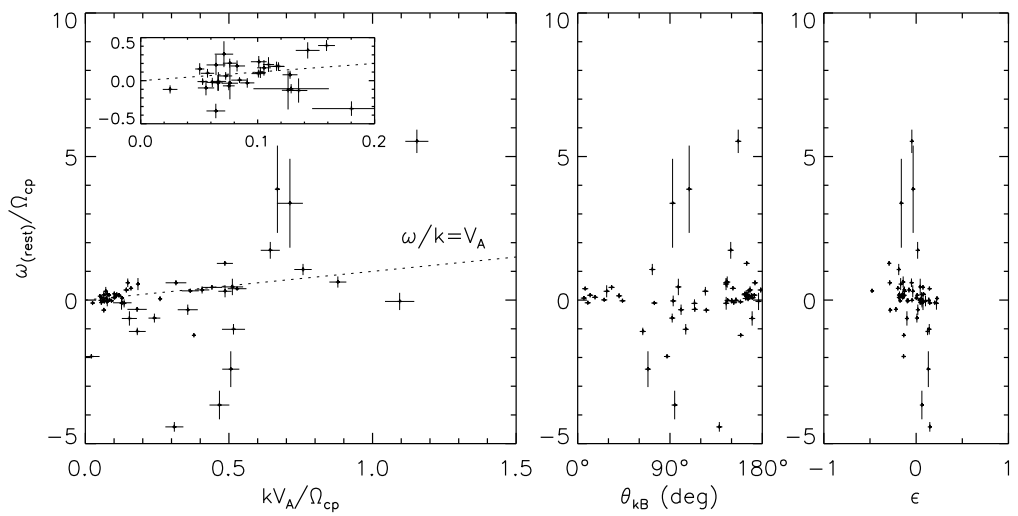

Figure 6.7: Distribution of rest frame frequencies, wave numbers, propagation angles, and ellipticities in the plasma rest frame with error bar estimates. The small plot embedded in the left panel is a magnified plot of the frequencies and the wave numbers near origin. The dotted, straight line in the left panel is a dispersion relation for the extended linear Alfvén waves (Narita et al. 2004).

\subsection{Discussion}

Upstream propagation is dominating in the foreshock, as is expected from earlier case studies. (Russell et al. 1971, Hoppe et al. 1981, Hoppe and Russell 1983, Eastwood et al. 2002, Narita et al. 2003). We also identified rest frame frequencies $\sim 0.1 \times \Omega_{c p}$ and wave numbers $\sim 0.1 \times \Omega_{c p} / V_{A}$ (wavelengths of the order of $R_{E}$ ). These results confirm the ISEE observations (Hoppe et al. 1981, Hoppe and Russell 1983) and imply propagation speeds close to Alfvén velocity. If we restrict the observations to propagation angle parallel/anti-parallel to the magnetic field at frequency much smaller than the proton cyclotron frequency, we may conclude that the dominant waves in the foreshock propagate at about the Alfvén speed. Taking account for the polarization analysis that waves are lefthanded rather than right-handed, our results prefer the beam ion instability theory.

Some of the minor waves, despite larger wave numbers, had phase velocities close to the Alfvén velocity, whereas non-magnetohydrodynamic waves such as the ion cyclotron resonant waves or the cold plasma waves might be expected in this domain. These minor waves tend to propagate perpendicular to the magnetic field. The waves propagating along the magnetic field in the foreshock region have been widely studied and discussed, but the perpendicular propagation may be important as well in understanding the physical processes in this region. 


\subsection{Summary}

Various properties of low-frequency waves such as frequencies, wave numbers, phase velocities, and polarization are investigated in the plasma rest frame in the upstream (foreshock) region. Using Cluster observations, the wave telescope is applied to investigate the wave numbers and the rest frame frequencies. One finds that most of the foreshock waves propagate upstream along the magnetic field at phase velocities close to the Alfvén velocity. We identify that frequencies are around $0.1 \times \Omega_{c p}$ and wave numbers around $0.1 \times \Omega_{c p} / V_{A}$. 
Table 6.1: Time intervals and dominant frequencies taken from 4-s and 1-s magnetic field data used in the statistical study of the foreshock waves .

\begin{tabular}{ccc}
\hline Interval & $\begin{array}{c}f_{\text {sc low })} \\
\mathrm{mHz}\end{array}$ & $\begin{array}{c}f_{\text {sc }(\text { high })} \\
\mathrm{mHz}\end{array}$ \\
\hline 2002-02-03 / 04:00-04:35 & 89.8 & 113.3 \\
2002-02-11 / 22:00-22:35 & 50.8 & 160.2 \\
2002-02-12 / 10:00-10:35 & 36.1 & 156.3 \\
2002-02-12 / 12:00-12:35 & 40.0 & 101.6 \\
2002-02-12 / 14:00-14:35 & 67.4 & 398.4 \\
2002-02-13 / 16:00-16:35 & 88.9 & 308.6 \\
2002-02-16 / 07:00-07:35 & 40.0 & 164.1 \\
2002-02-18 / 08:05-08:40 & 44.9 & 312.5 \\
2002-02-20 / 17:00-17:35 & 55.7 & 97.7 \\
2002-02-20 / 22:00-22:35 & 50.8 & 101.6 \\
2002-02-21 / 22:00-22:35 & 62.5 & 246.1 \\
2002-02-26 / 20:30-21:05 & 44.9 & 269.5 \\
2002-03-01 / 07:00-07:35 & 47.9 & 214.8 \\
2002-03-06 / 00:30-01:05 & 36.1 & 433.6 \\
2002-03-07 / 05:00-05:35 & 39.0 & 97.7 \\
2002-03-09 / 12:00-12:35 & 30.3 & 425.8 \\
2002-03-09 / 14:20-14:55 & 25.4 & 97.7 \\
2002-03-09 / 16:00-16:35 & 27.3 & 175.8 \\
2002-03-10 / 17:00-17:35 & 42.0 & 97.7 \\
2002-03-11 / 20:00-20:35 & 46.9 & 210.9 \\
2002-03-13 / 04:30-05:05 & 34.1 & 320.3 \\
2002-03-16 / 13:00-13:35 & 35.1 & 97.7 \\
2002-03-16 / 15:00-15:35 & 30.3 & 140.6 \\
2002-03-26 / 17:50-18:25 & 29.3 & 453.1 \\
2002-03-27 / 07:00-07:35 & 43.0 & 335.9 \\
2002-03-29 / 18:00-18:35 & 114.3 & 308.6 \\
2002-03-29 / 20:30-21:05 & 55.7 & 390.6 \\
2002-04-02 / 04:00-04:35 & 81.1 & 160.1 \\
2002-04-03 / 16:30-17:05 & 21.5 & 457.0 \\
2002-04-27 / 02:00-02:35 & 43.9 & 97.7 \\
2002-05-07 / 23:20-23:55 & 34.2 & 113.3 \\
2002-05-13 / 14:50-15:25 & 50.8 & 457.0 \\
& 44.9 & 457.0 \\
20 06:00-06:35 & 37.1 & 406.3
\end{tabular}


2002-05-20/08:30-09:05 $44.9 \quad 312.5$

2002-05-22 / 11:20-11:55 41.0 457.0 



\section{Dispersion analysis of downstream waves}

\subsection{Introduction}

In any experiments it is often said that case studies and statistical studies are complementary to each other. The former method provides detailed information about what happens in the selected case or event, while the latter identifies various populations that have distinct aspects. Those methods applied to the upstream waves proved that the waves represent the one driven by the (right-hand) ion beam instability and propagating toward upstream, where the beam is provided by the ion reflection at the shock and backstreaming against the incoming flow. According to the statistics in the previous chapter the majority of the upstream waves exhibits this property.

Let us move onto the downstream region and apply those methods there. It is also of interest to ask how the upstream and the downstream waves are related to each other. It should be noted that this problem is not solved yet, for example, whether the upstream waves enter the magnetosheath as they are carried by the solar wind. Early studies on the wave transmission across the bow shock also motivate this study. In the magnetohydrodynamic (MHD) picture a wave incident on the front side of the bow shock gives rise to different kinds of MHD waves which diverge from the back of the shock: Alfvén waves, magnetosonic waves, and an entropy wave. Their respective amplitudes are given by solving the perturbed conservation relations across the shock. For example, an Alfvén wave upstream of the shock is transmitted into the downstream region with amplitude enhanced by a factor about three (McKenzie and Westphal 1969, 1970, Hassam 1978) and an upstream magnetosonic wave is also enhanced across the shock (McKenzie and Westphal 1969). Krauss-Varban and Omidi (1991, 1993) and Krauss-Varban (1994), on the other hand, presented hybrid simulations of upstream and downstream waves at the quasi-parallel shock and argued that the upstream waves driven by the right-hand ion beam instability are mode converted into the Alfvén/ion cyclotron waves. As pointed out by Krauss-Varban et al. (1994b), when the scale of the waves approaches ion scales, a kinetic treatment is necessary.

In this chapter we address the question of how wave dispersion characteristics are changing when going from the upstream region through the bow shock toward the magnetopause. A detailed characterization of wave dispersion properties is aimed for as 


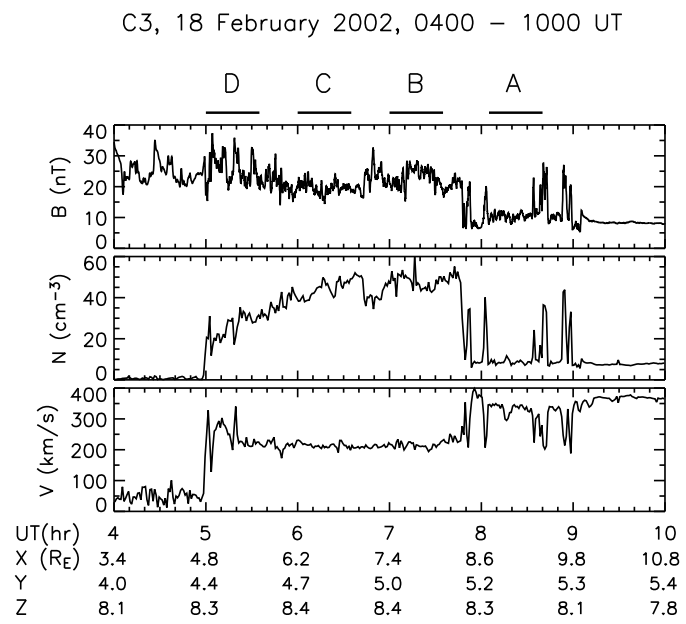

Figure 7.1: Time series plot of magnetic field strength, ion density, and ion bulk velocity observed by Cluster. The time interval shown is the same as Fig. 6.1 (Narita and Glassmeier 2005).

they allow to discriminate between, for example, transmitted waves and locally generated waves. Also, waves excited by the ion beam instability usually exhibit different dispersion branches as theoretical studies indicate. Which branch is important can easily be decided studying experimentally determined dispersion relations (Chapter 4).

Dispersion relations are determined from the magnetic field and ion measurements made by Cluster. Using a Fourier analysis in the time domain and the wave telescope allows one to construct $\omega$ - $k$-diagrams (frequency - wave number diagrams) in the plasma rest frame corrected for the Doppler shift.

For the present study we use data from the interval 0400-1000 UT on February 18, 2002, when the spacecraft separation was about $100 \mathrm{~km}$. Fig. 7.1 displays the magnetic field strength as well as the ion density and the bulk velocity. The Cluster s/c moved outbound from the magnetosphere into the solar wind. The interval 0400-0500 UT covers observations in the Earth's magnetosphere, 0500-0800 UT the magnetosheath, 0800-0900 UT the foreshock with several shock crossings, and 0900-1000 UT covers the solar wind (Fig. 7.2). We select four subintervals for the dispersion analysis: 0805-0840 UT for the upstream (foreshock) waves (labeled as A), 0700-0735 UT the outer magnetosheath waves (B), 0600-0635 UT the middle magnetosheath waves (C), and 0500-0535 UT the inner magnetosheath waves (D). The shock angle determined using the magnetic field coplanarity theorem is $17^{\circ}$, that is the Cluster s/c traversed a quasi-parallel shock. 


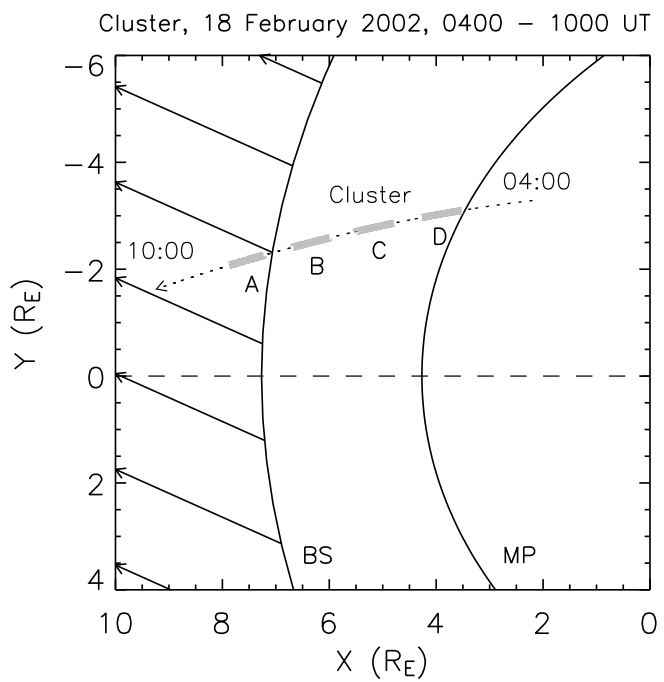

Figure 7.2: Cluster orbit in the plane made by the solar wind direction (opposite to the $x$-axis in the plot) and interplanetary magnetic field direction. BS and MP stand for the bow shock and the magnetopause in this plane, respectively. The solid arrows represent the interplanetary magnetic field direction, and the solid arrow represents the Cluster orbit (Narita and Glassmeier 2005).

\subsection{Dispersion analysis}

The analysis method follows Chapter 4 . The wave power is calculated in the wave number domain at various frequencies using the wave telescope. Magnetic field data with 1s time resolution are used in the analysis and the wave power is investigated at wavelengths down to $200 \mathrm{~km}$. A Doppler shift of frequency is then corrected for the relation

$$
\omega_{\text {rest }}=\omega_{\text {sc }}-\boldsymbol{k} \cdot \boldsymbol{V}_{\text {flow }},
$$

where $\omega_{\text {rest }}, \omega_{s c}, \boldsymbol{k}$, and $\boldsymbol{V}_{\text {flow }}$ denote the wave angular frequency in the plasma rest frame of reference and in the spacecraft frame, wave vector, and plasma flow velocity vector, respectively. For the plasma flow velocity we use the ion bulk velocity data measured by CIS-HIA, assuming that the background flow is stationary. It is worthwhile to note that one needs to know the full wave vector to obtain the frequency in the plasma rest frame. Only multi-point measurement of the Cluster $\mathrm{s} / \mathrm{c}$ are up to now able to provide this information.

In addition to the dispersion analysis we use a polarization analysis to identify the wave modes. The transverse magnetic field polarization $p$ is defined as (Fowler et al. 
1967)

$$
p=\tan \phi=i \frac{\delta B_{y}}{\delta B_{x}}
$$

where $\phi$ denotes the polarization angle determined from the cross spectral density matrix of the transverse magnetic field fluctuations, and $\delta B_{y}$ and $\delta B_{x}$ denote the minimum and maximum transversal magnetic field variation, respectively. A value $p=+1$ indicates right-hand circular polarization with respect to the magnetic field direction, that is in the same sense as the gyromotion of an electron, $p=0$ linear polarization, and $p=-1$ lefthand circular polarization, that is the same rotation sense as the ion gyromotion. Because $p$ depends on frequency (Stix 1992), the sign of polarization is changed when the rest frame frequency is negative (anomalous Doppler shift).

The results of the dispersion and polarization analysis are displayed in Fig. 7.3. The difference of the background magnetic field strength and the plasma density between the foreshock and the magnetosheath results in difference of normalized frequency - wave number range available: a higher Alfvén speed in the magnetosheath than in the foreshock results in a smaller value of normalized wave number. Due to the anomalous Doppler shift the signs of the rest frame frequencies are reversed to bring most of them into the positive regime in all the panels. Accordingly, the propagation angle is changed as $\theta_{k B} \rightarrow$ $180^{\circ}-\theta_{k B}$. For scanning in the $k$-space we use a spherical grid with $75 \times 60 \times 60$ points in radial, azimuthal, and polar direction up to Nyquist wave number $k_{N y}=3 \times 10^{-2} \mathrm{~km}^{-1}$. The uncertainty of wave number is thus about $\Delta k=4 \times 10^{-4} \mathrm{~km}^{-1}$ for the wave number magnitude and $\Delta \theta_{k B}=3^{\circ}$ for the propagation angle with respect to the magnetic field. The uncertainty in the rest frame frequency represents the one in the Doppler shift, i.e. $\left|\frac{\Delta \omega_{r e}}{\omega_{r e}}\right| \leq\left|\frac{\Delta k}{k}\right|+\left|\frac{\Delta V}{V}\right|$. We use $\Delta V$ which is calculated from mean absolute deviation of the CIS-HIA bulk velocity data. The results of the polarization analysis are over-plotted. We classify the polarization as right-handed (RH) if $p>0.176$ (i.e. $\phi>10^{\circ}$ ), left-handed (LH) if $p<-0.176$ (i.e. $\phi<-10^{\circ}$ ), and linear (Lin) if $-0.176 \leq p \leq 0.176$.

The upstream waves exhibit two branches [Fig. 7.3A]. Branch 1 starts at $(\omega, k) \simeq$ $(0,0)$ and extends up to about $(\omega, k) \simeq(1.5,1.5)$. Branch 2 reaches from $(\omega, k) \simeq$ $(-1.0,0.0)$ to about $(\omega, k) \simeq(0.5,0.2-0.3)$, intersecting branch 1 at $(0.1,0.1-0.2)$. Propagation is slightly off-angle at $20^{\circ}-30^{\circ}$ for almost all wave numbers. Only for very small wave numbers it is perpendicular to the background magnetic field. Various polarizations are detected with the linear and the right-hand polarization dominating (Fig. 7.4A).

In the outer magnetosheath (near the shock) frequencies are small (at most 0.4) at various wave numbers (Fig. 7.3B). Propagation is oblique to perpendicular with a few nearly parallel cases $\left(12^{\circ}<\theta_{k B}<20^{\circ}\right)$; the polarization is clearly linear (Fig. 7.4B). The nearly parallel propagating waves exhibit phase speeds about $0.1-0.2$ as large as the Alfvén speed with left-hand polarization $(-0.2<p<-0.6)$, which may represent ion cyclotron waves.

In the middle magnetosheath the dispersion appears as an almost horizontal line at about zero frequency, though the frequencies deviate a little from zero at wave numbers 

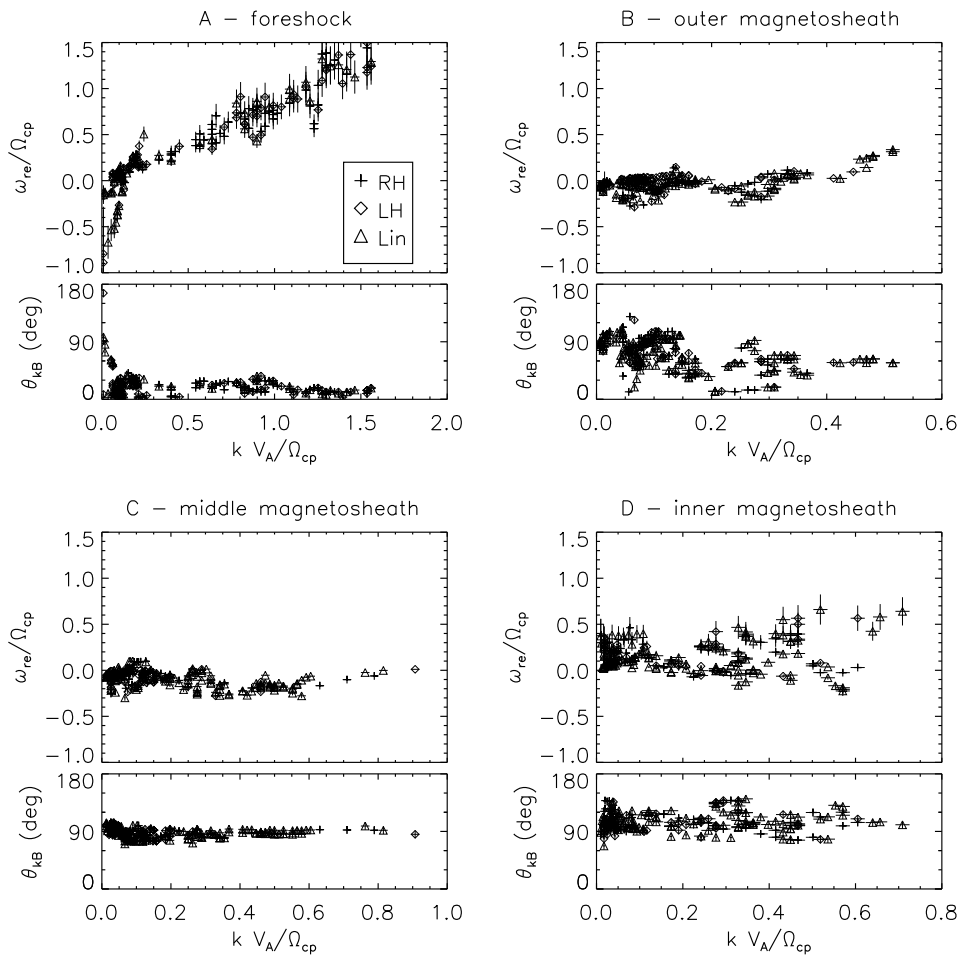

Figure 7.3: Dispersion relations and propagation angles with error bars in the foreshock (0805-0840 UT), in the outer magnetosheath (0700-0735 UT), in the middle magnetosheath (0600-0635 UT), in the inner magnetosheath (0500-0535 UT). Frequency and wave number are normalized to proton cyclotron frequency $\Omega_{c p}$ and inverse of ion inertial scale $\Omega_{c p} / V_{A}$, where $V_{A}$ stands for Alfvén velocity. The quantities are $\Omega_{c p}=0.92$ (rad $\mathrm{Hz})$ and $V_{A}=61.84(\mathrm{~km} / \mathrm{s})$ for in the foreshock (A), $\Omega_{c p}=1.99(\mathrm{rad} \mathrm{Hz})$ and $V_{A}=59.56$ $(\mathrm{km} / \mathrm{s})$ in the outer magnetosheath (B), $\Omega_{c p}=1.84(\mathrm{rad} \mathrm{Hz})$ and $V_{A}=57.50(\mathrm{~km} / \mathrm{s})$ in the middle magnetosheath (C), and $\Omega_{c p}=2.01(\mathrm{rad} \mathrm{Hz})$ and $V_{A}=82.53(\mathrm{~km} / \mathrm{s})$ in the inner magnetosheath (D) (Narita and Glassmeier 2005).

$0.4-0.6$ (Fig. 7.3C). Wave vectors are clearly perpendicular to the magnetic field and predominantly oriented in the direction from the sun to the Earth $\left(k_{x}<0\right.$ in the GSE coordinate system); the polarization is linear (Fig. 7.4C).

The inner magnetosheath waves (near the magnetopause) exhibit scattered frequencies and propagation angles (Fig. 7.3D). Average propagation angles tend to be $90^{\circ}-120^{\circ}$; 

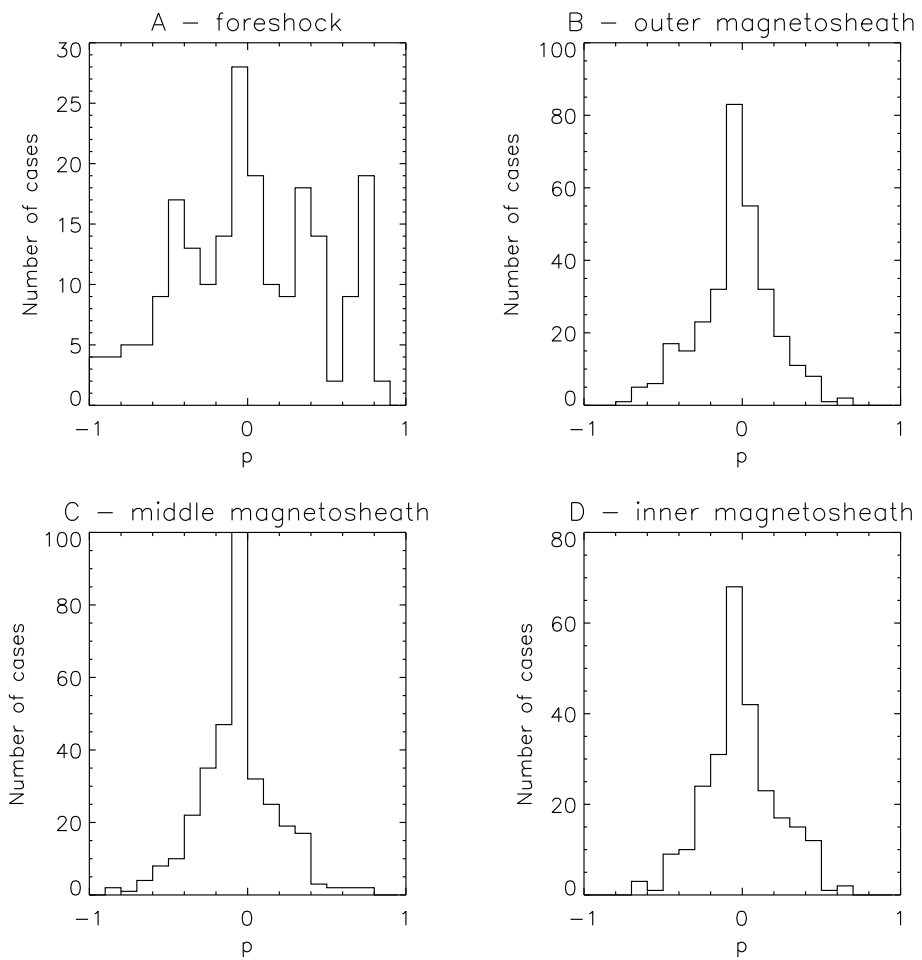

Figure 7.4: Histograms of magnetic field polarization at various frequencies for the same events as Fig. 7.3 (Narita and Glassmeier 2005).

the polarization is still clearly linear (Fig. 7.4D).

\subsection{Discussion}

Two intersecting branches in the upstream wave dispersion were already identified in Chapter 4 . The first branch represents the right-hand whistler mode and the second branch the beam resonant mode in which the wave is propagating together with the ion beam. Polarization is found to be linear to right-hand, which is also in accord with the whistler branch.

In the outer magnetosheath these properties are lost except for the perpendicular propagation at very small wave numbers. Mirror modes are dominating in the middle mag- 
Table 7.1: Plasma $\beta$, temperature anisotropy $T_{\perp} / T_{\|}$, and transport ratio $R=\cos \psi_{n b}$ for different regions investigated, where $\psi_{n b}$ denotes the phase angle between the plasma density and the magnetic field fluctuations (Narita and Glassmeier 2005).

\begin{tabular}{lccc}
\hline Region & $\beta$ & $T_{\perp} / T_{\|}$ & $R$ \\
\hline Foreshock & 4.838 & 0.702 & 0.974 \\
Outer Sheath & 5.982 & 1.229 & -0.977 \\
Middle Sheath & 8.012 & 1.258 & -0.893 \\
Inner Sheath & 3.425 & 1.217 & -0.885 \\
\hline
\end{tabular}

netosheath, since the frequencies are almost zero, the wave vectors are perpendicular to the background magnetic field, polarization is linear, and furthermore, the fluctuations of the magnetic field are anti-correlated to the plasma density fluctuations (Table 7.1). The outer magnetosheath waves seem to be in a transient state toward the mirror mode. There may exist waves other than the mirror mode in the outer magnetosheath that fade toward the middle magnetosheath. Hubert et al. (1998) argued using ISEE observations that the magnetosheath waves represent the ion cyclotron waves near the shock and the mirror mode structures closer to the magnetopause. Denton et al. (1998) also presented Alfvénlike modes in the outer magnetosheath. The ion cyclotron waves are a likely candidate. We speculate that the outer magnetosheath waves are a mixture of the mirror modes and ion cyclotron waves propagating toward the flank region.

In the inner magnetosheath the mirror mode properties are distorted such that frequencies (and also phase speeds) deviate from zero and propagation angles deviate from $90^{\circ}$, while they keep the linear polarization. The mirror modes with finite frequencies and phase speeds were already found by Omidi and Winske (1995). They used a onedimensional hybrid simulation to model the entire region from the solar wind to the magnetic boundary and found mirror mode waves that are phase standing relative to the magnetic boundary, which implies that the phase standing waves must have a finite propagation speed in the plasma rest frame. These waves are referred to as 'MIrror And slOW' or MIAOW waves and observed near the magnetopause Balikhin et al. (2001). The vicinity of the magnetopause seems to modify the mirror mode characteristics. The finite propagation of the mirror mode in oblique direction may be due to a variety of processes, including nonlinearity (Stasiewicz 2004a), inhomogeneous background state such as density gradients (Hasegawa 1969, Pokhotelov et al. 2001), or eigenmode of the magnetosheath (Johnson and Cheng 1997).

Table 7.1 displays plasma parameter $\beta$ (ratio of thermal to magnetic pressure, $p_{t h} / p_{B}$ ), temperature anisotropy $T_{\perp} / T_{\|}$, and transport ratio $R$ for the time intervals under investigation. Plasma $\beta$ is greater than unity (thermal pressure dominant) throughout the intervals, though it shows a variation, being enhanced from about 4 in the foreshock to about 8 toward the middle magnetosheath and diminished to about 3 in the inner magnetosheath. The enhancement of $\beta$ in the middle magnetosheath stems from the diminished IMF by 


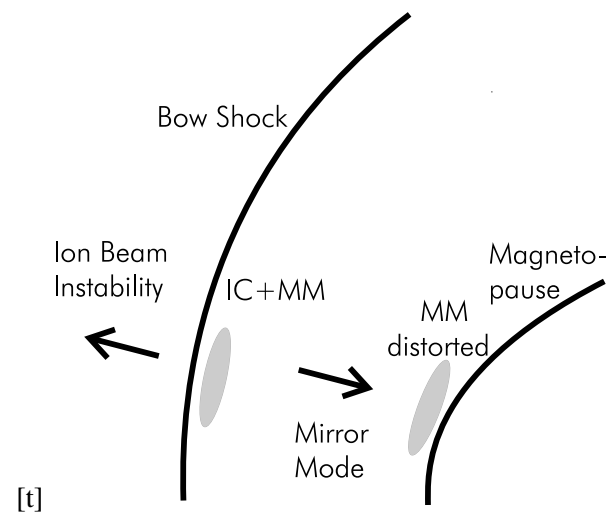

Figure 7.5: Schematic illustration of wave habitats. Arrows represent wave vectors. 'IC' and 'MM' stand for ion cyclotron waves and mirror modes, respectively (Narita and Glassmeier 2005).

about $20 \%$ detected by the ACE spacecraft. Temperature anisotropy $T_{\perp} / T_{\|}$, which is ratio of perpendicular to parallel temperature with respect to the background magnetic field direction, becomes parallel dominant (about 0.7) in the foreshock to perpendicular dominant in the magnetosheath (about 1.2). Anisotropy is nearly constant in the magnetosheath. Transport ratio $R$ represents the cosine of the phase angle between the density and magnetic field strength variations (Denton et al. 1998), and it is averaged over frequencies under the condition of the coherence greater than 0.7 between the two variations. It shows the value about 1 (correlated density and magnetic field variations) in the foreshock and -1 (anti-correlated variations) in the magnetosheath. The foreshock waves exhibit the property of the fast magnetosonic waves which is consistent with the right-hand beam instability and that the magnetosheath waves exhibit predominantly the mirror mode characteristics. The mirror mode interpretation in the magnetosheath is also supported by the high plasma $\beta$ and the large perpendicular temperature observed.

Fig. 7.5 illustrates wave habitats and wave vectors derived from our study. The upstream waves are driven by the ion beam instability with wave vectors oriented toward upstream and almost parallel to the background magnetic field. In the outer magnetosheath there are a minor contribution of the ion cyclotron waves (IC) and a major contribution of the mirror modes (MM). The middle magnetosheath exhibits the clearest mirror modes. In the inner magnetosheath the mirror mode properties are distorted probably due to additional effects such as nonlinear, inhomogeneous background, or eigenmode effects.

From the viewpoint of the problem of the wave transmission or mode conversion across the shock, we find that the fast mode is not present in the downstream region and that the ion cyclotron waves do not dominate either. The mirror modes and the ion cy- 
clotron waves in the magnetosheath are believed to originate in the temperature anisotropy provided by the ion reflection at the shock, and it seems that the wave transmission or mode conversion across the shock does not apply to the Earth's bow shock case. But we note that Krauss-Varban and Omidi (1993) used a low plasma $\beta$ condition for their simulation study, while high $\beta$ dominates in the present case. Therefore, it is worthwhile to do a numerical simulation under the high $\beta$ condition and to compare with our result, which will be a subject of future investigation.

\subsection{Summary}

The dispersion analysis and additional investigations on the polarization and the transport ratio suggest that the upstream waves lose their identity as they are convected by the background flow and that they are not transmitted into the magnetosheath. Perhaps they are lost near or at the shock. In the outer magnetosheath the mirror modes and the ion cyclotron waves are likely to coexist, both of which are known to be driven by pressure anisotropy. In the middle magnetosheath only the mirror modes dominate, while in the inner magnetosheath their properties are distorted to have finite frequencies and wave vectors oblique to the magnetic field. The mirror modes are subject to nonlinear or inhomogeneous effect near the magnetopause. Further investigations on dispersion relation, propagation and polarization characteristics, and transport ratios in various regions of the foreshock and the magnetosheath will verify our result. On the other hand, theoretical investigations under various conditions (e.g. high plasma $\beta$ ) will improve our comprehension about the mode transmission or conversion problem. 



\section{Statistical study of downstream waves}

\subsection{Introduction}

The dispersion analysis presented in Chapter 7 suggests that the mirror modes dominate in the downstream region, but on the other hand it has been understood that their frequencies are not exactly zero. This chapter presents a statistical study of the downstream waves and attempts to determine typical values of wave frequencies, wave number, and so on, for different regions from the upstream to the downstream region, and for the quasi-parallel and the quasi-perpendicular shock regime.

The propagation pattern presented in Chapter 6 is also extended to the downstream region. Matsuoka et al. (2000) and Schäfer et al. (2005) showed that Alfvénic waves in the magnetosheath propagate in the same direction as the plasma flow in a draped field, that is anti-sunward and away from the sun-Earth line. Yet, little is known about how the waves propagate at what speed nor whether the propagation properties are similar or different between the quasi-parallel and the quasi-perpendicular shock regimes. This stems from the fact that wave vectors were hardly determined in single or at best two spacecraft observations, preventing one from determining phase velocities.

\subsection{Statistical study}

In order to perform the statistical study the following criteria and methods are used. About 50 upstream events and about 200 downstream events observed by the Cluster s/c are investigated. Since the selection procedure for the upstream wave events was already presented in the foreshock wave study (Chapter 6), we describe here the downstream wave events in detail.

The mission phase with a $100 \mathrm{~km}$ spacecraft separation, that is the time interval from February 3 to June 17, 2002 is selected to resolve wavelengths down to $200 \mathrm{~km}$. The Cluster s/c observed in the post-noon to the dawn sector of the magnetosheath during this mission phase.

The magnetosheath region has been identified from bow shock and magnetopause crossings, by investigating magnetic field data from FGM instrument and ion data ob- 


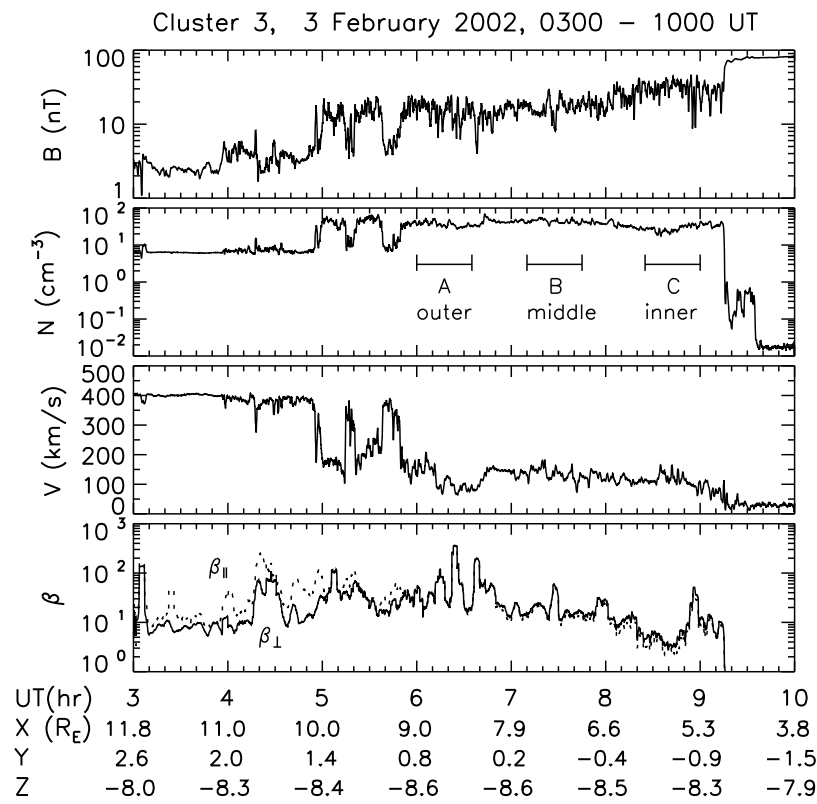

Figure 8.1: Time series plot of magnetic field strength, ion number density, ion bulk speed, and plasma beta parallel (dotted) and perpendicular (dashed) to the magnetic field, observed by Cluster 3 from 03:00 UT (solar wind region) to 10:00 UT (magnetosphere), February 3, 2002 (Narita et al. 2006a).

tained by CIS-HIA and CIS-CODIF instrument. Identification of the bow shock crossing for the inbound spacecraft motion is based on a sudden increase of the magnetic field within the spacecraft spin period ( $4 \mathrm{~s}$ ) by a factor $2-4$, accompanied by an increase of the ion density, and a decrease of the ion bulk speed. For the inbound magnetopause crossing an increase of the magnetic field, a significant decrease of the ion density and a moderate decrease of the ion bulk speed are used as an indicator. Fig. 8.1 displays as an example the magnetosheath observations made on February 3, 2002. The spacecraft motion was inbound from the solar wind to the magnetosphere.

Three intervals are chosen as the outer, the middle, and the inner magnetosheath region from each traversal with the intervals equidistantly positioned in the time domain. Fig. 8.1 displays an example of the interval selection. For the outer and the inner magnetosheath intervals as close as possible to the bow shock and to the magnetopause, respectively, have been selected. During some orbits only either the bow shock or the magnetopause 
crossing could be identified due to a lack of data. In such a case we drop the inner or the outer region and pick up the remaining two intervals to analyze as many events as possible. As a result of the selection procedure we obtain 84 magnetosheath traversals with 197 intervals for the statistical study (Table 8.1).

To determine whether the observations are made closer to the quasi-parallel or the quasi-perpendicular shock we introduce a new coordinate system, the solar wind system, in which the basis vectors $\boldsymbol{e}_{x}, \boldsymbol{e}_{y}$, and $\boldsymbol{e}_{z}$ are given by

$$
\begin{aligned}
\boldsymbol{e}_{x} & =-\boldsymbol{e}_{v} \\
\boldsymbol{e}_{y} & =\boldsymbol{e}_{z} \times \boldsymbol{e}_{x} \\
\boldsymbol{e}_{z} & =\frac{B_{s}}{\left|B_{s}\right|} \boldsymbol{e}_{v} \times \boldsymbol{e}_{b},
\end{aligned}
$$

where $e_{v}$ and $e_{b}$ are the unit vectors of the solar wind velocity and the interplanetary magnetic field (IMF), and $B_{s}$ is the sunward component of the IMF. Fig. 8.2 shows two examples of how the coordinate system is transformed. First the $x$-axis is oriented antiparallel to the solar wind direction, then the system is rotated about the $x$-axis so that the $x y$-plane is identical with the plane spanned by the solar wind and the IMF directions. There are two possibilities to determine the $y$-direction, closer to or distant from the IMF direction. We choose the $y$-direction which is oriented closer to the IMF direction if $B_{s}<0$ (Fig. 8.2 top) and to the opposite direction if $B_{s}>0$ (Fig. 8.2 bottom), so that the IMF line is always tangential to the bow shock in the $y>0$ regime. To determine the solar wind and the IMF directions, ion and magnetic field data from the ACE s/c (Stone et al. 1998) are used. Since ACE monitors the solar wind at a distance of about 200 to $250 R_{E}\left(1 R_{E}=6370 \mathrm{~km}\right)$ in front of the Earth, its observation time is shifted forward by 60 minutes to compensate any time delay between the ACE position and the Earth. The ACE data are averaged over 90 minutes. We associate the observations with the near quasi-perpendicular shock regime if the sign of the $y$-component of the Cluster position in the newly introduced solar wind coordinate system is positive and vice versa. This coordinate system is equivalent to the three axes of the magnetosheath interplanetary medium (MIPM) reference frame (Verigin et al. 2006). The advantage to use this coordinate system is that one can determine the shock regime corresponding to given Cluster observations even if shock crossing data are not available.

Fig. 8.3 shows an example of the Cluster orbit in the solar wind system for the same interval as Fig. 8.1. The Cluster trajectory is located in the near quasi-parallel shock regime. Note that this classification is based on determination of the upstream conditions and does not work if the IMF cone angle is close to $0^{\circ}$ or $90^{\circ}$ and that it is different from the one based on the shock angle determination using the magnetic field coplanarity theorem, although both results agree well with each other in the statistical study. In the case displayed in Fig. 8.3, we obtain $\theta_{B n}=13^{\circ}$ (quasi-parallel shock) using the coplanarity theorem for the shock crossing, where the Alfvén Mach number is about 18.

For any of the events the dominant frequency is determined using the power spectrum of the magnetic field in the spacecraft frame. We use 1s- and 4s-averaged magnetic field 

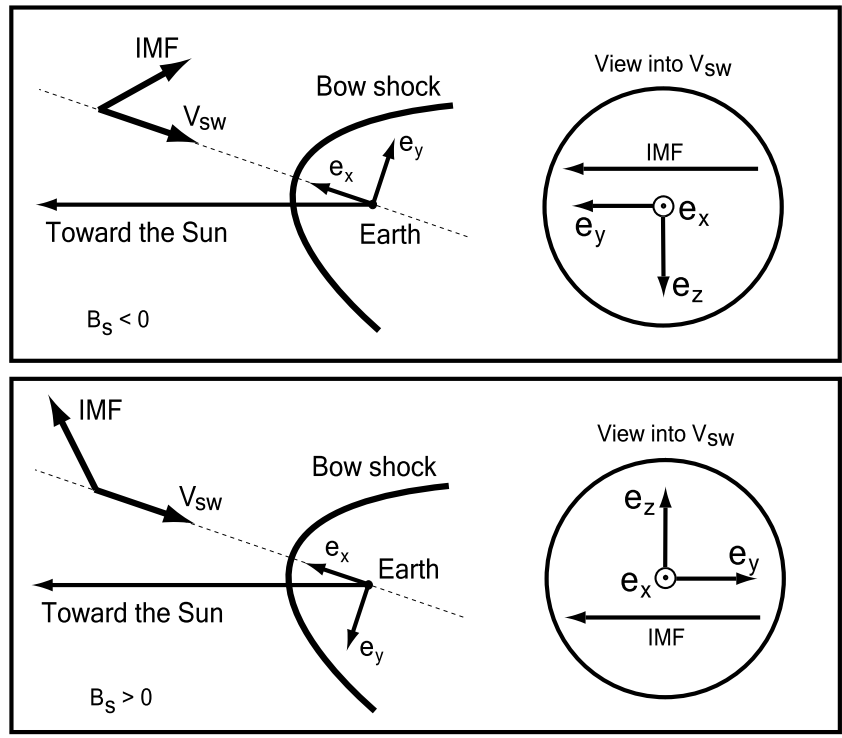

Figure 8.2: Two sketches of the solar wind coordinate system for IMF with a negative sunward component $\left(B_{s}<0\right)$ and with a positive component $\left(B_{s}>0\right)$ (Narita et al. 2006a).

data. The 4s-data are used to compare with CIS observations available with the corresponding time resolution. Fig. 8.4 shows the wave power in the frequency domain for the example case. The wave power $P(f)$ is compared with the power law $P \propto f^{-\alpha}$ (reference wave power), where $P$ is the wave power, $f$ is the frequency, and $\alpha$ is a positive value determined by fitting of the spectrum. From the power spectrum the frequency is identified as the dominant frequency where the measured wave power deviates most from the reference wave power. In the example case we obtain $41 \mathrm{mHz}$.

The polarization about the magnetic field is investigated. We define the magnetic polarization $p$ following the ellipticity defined by Fowler et al. (1967), which is calculated from the cross spectral density matrix. In the investigation the basis vectors of the cross spectral density matrix of the magnetic field fluctuation are oriented to the direction of principal and minimal magnetic field variation in the plane perpendicular to the background magnetic field. A value of $p=1$ denotes the right-hand circular polarization when viewed into the magnetic field direction, $p=0$ the linear polarization and $p=-1$ the left-hand polarization. Under this definition a right-hand mode propagating either parallel or anti-parallel to the magnetic field possesses fluctuating field vectors that rotate in the same sense as the gyro-motion of an electron (Eq. 7.2). 


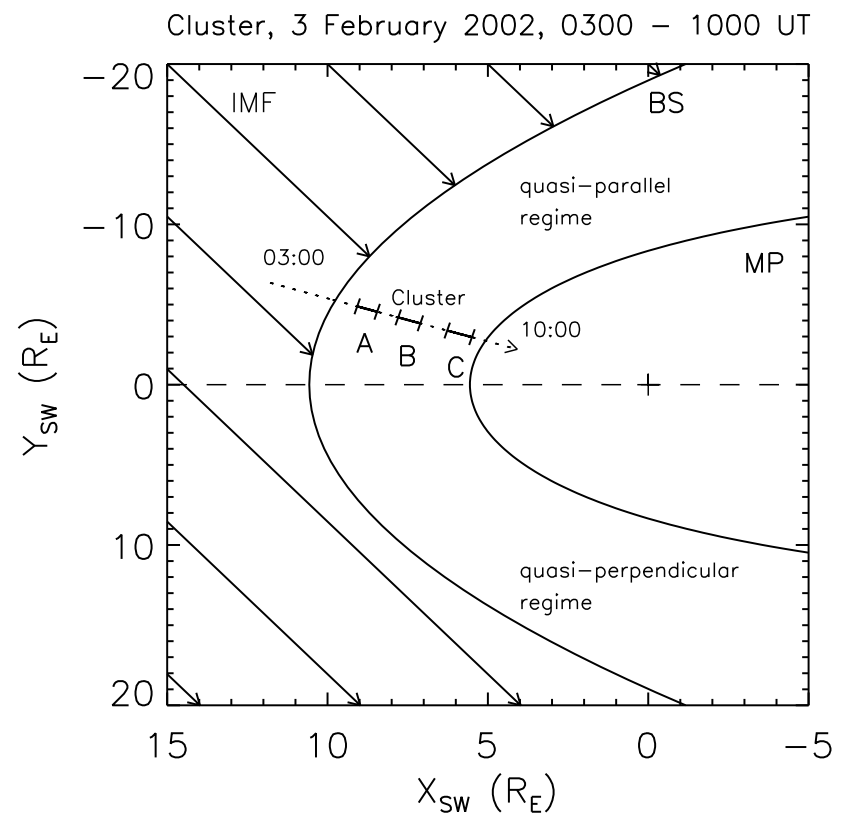

Figure 8.3: Cluster orbit in the solar wind coordinate system for the interval in Fig. 8.1. ' $A$ ', 'B' and ' $C$ ' represent the outer, the middle and the inner magnetosheath that are selected for the wave analysis. BS, MP, and IMF stand for the bow shock, the magnetopause, and the interplanetary magnetic field, respectively (Narita et al. 2006a).

We investigate transport ratios which are the coherence $\gamma$ and the phase angle $\phi$ for the density and the magnetic field variations, and for the flow velocity and the magnetic field variations at the dominant frequency. In the example case we obtain $\gamma=0.99$ and $\phi=179^{\circ}$ for the former transport ratio, $\gamma=0.49$ and $\phi=33^{\circ}$ for the latter one. As Fig. 8.5 visually indicates, the density and the magnetic field variations are anticorrelated. Note that, as Reiff (1983) pointed out, it is square of correlation or coherence that measures the fraction of the two variances which can be explained by the linear model. Thus, although $\gamma=0.5$ may sound like a good fit, it means that as much as $75 \%$ of the variance is still unexplained.

The wave number vector $k$ associated with the dominant frequency is determined from the wave telescope technique that requires multi-spacecraft measurements such as Cluster. This technique provides the wave power as a function of frequency and wave number with the assumption that the data analyzed represent stationary and homogeneous fluctuations. 


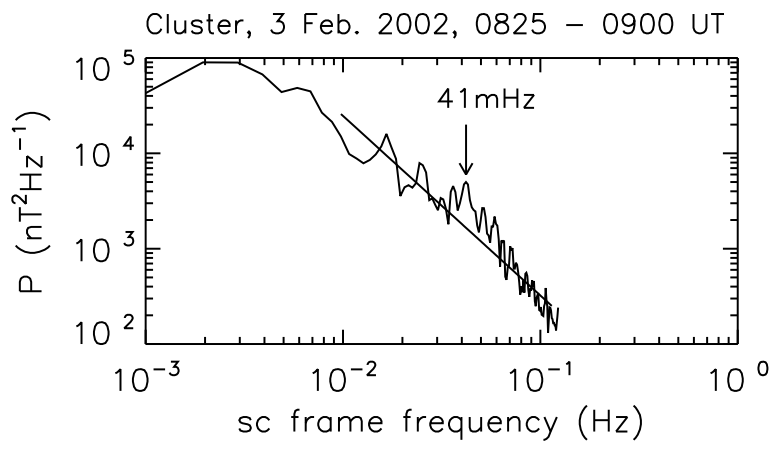

Figure 8.4: Wave power as a function of spacecraft frame frequency calculated from magnetic field data of Cluster 3 for the interval $\mathrm{C}$ in Fig. 8.1. The straight line shows the wave power fitted by the power law $P \propto f^{-\alpha}$. The dominant frequency is found at 41 $\mathrm{mHz}$ (Narita et al. 2006a).

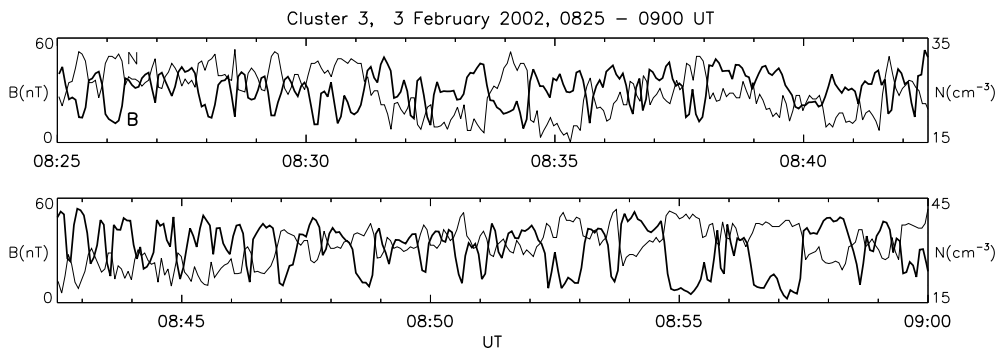

Figure 8.5: Time series plot of the magnetic field magnitude (thick line) and the ion number density (thin line) measured by Cluster 3 for the interval $\mathrm{C}$ in Fig. 8.1. A clear anticorrelation between the two fluctuations indicates the mirror mode in the magnetosheath (Narita et al. 2006a).

Fig. 8.6 displays the wave power in the $k$-domain for the example case. Maximum power is detected at $\left(k_{\|}, k_{\perp}\right)=\left(-3.67 \times 10^{-4}, 3.64 \times 10^{-3}\right) \mathrm{km}^{-1}$ at a wavelength of 1700 $\mathrm{km}$, and the angle $\theta_{k B}$ between the wave vector $\boldsymbol{k}$ and the magnetic field is $96^{\circ}$ (cf. ion inertial length is $270 \mathrm{~km}$ and thermal ion gyro-radius is $489 \mathrm{~km}$ for the interval under investigation). 


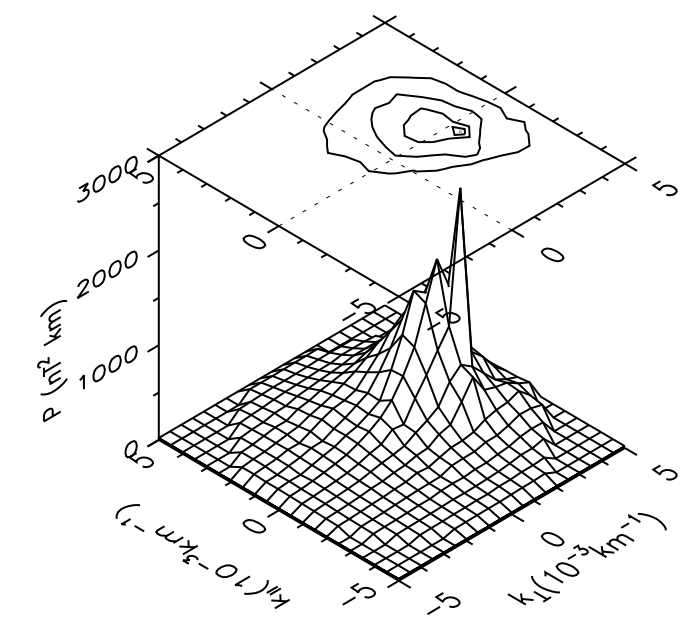

Cluster, 3 February 2002, 0825 - 0900 UT, 41.0 mHz

Figure 8.6: Wave power as a function of wave number, parallel and perpendicular to the mean magnetic field, at spacecraft frame frequency $41 \mathrm{mHz}$. The wave power is calculated by the wave telescope technique, and peaks in the perpendicular direction. (Narita et al. 2006a).

It should be noted here that a proper Doppler shift correction requires knowledge of the wave vector $\boldsymbol{k}$. Only observations from the four Cluster s/c allow to determine $\boldsymbol{k}$ unambiguously with techniques such as the wave telescope. Using the Doppler shift relation $\omega_{\text {rest }}=\omega_{s c}-\boldsymbol{k} \cdot \boldsymbol{V}_{\text {flow }}$ we calculate the rest frame frequency. Here $\omega_{\text {rest }}$ and $\omega_{s c}$ denote the angular frequency in the rest frame and in the spacecraft frame, respectively, and $\boldsymbol{V}_{\text {flow }}$ the plasma flow velocity. CIS measurements of the ion bulk velocity are used to determine $\boldsymbol{V}_{\text {flow. }}$. We then calculate the wave phase velocity in the rest frame via $\boldsymbol{V}_{p h}=\omega_{\text {rest }} \boldsymbol{k} / k^{2}$. In our example case we obtain $\omega_{\text {rest }}=13 \mathrm{rad} \mathrm{mHz}$ and $\boldsymbol{V}_{p h}=(-0.3,3.4) \mathrm{km} / \mathrm{s}$ parallel and perpendicular to the background magnetic field, or $(-3.4,0.0,-0.7) \mathrm{km} / \mathrm{s}$ in the GSE system. These quantities are intrinsic and not influenced by convection due to the background plasma flow.

Upstream waves are investigated in the same way as described above for the magnetosheath waves. The waves upstream of the quasi-parallel shock represent the foreshock waves. Based on the event selection criteria in the foreshock wave study (Chapter 6) we identify 36 events for the foreshock (Table 6.1) and 19 events for the quasi-perpendicular shock upstream waves (Table 8.2). 

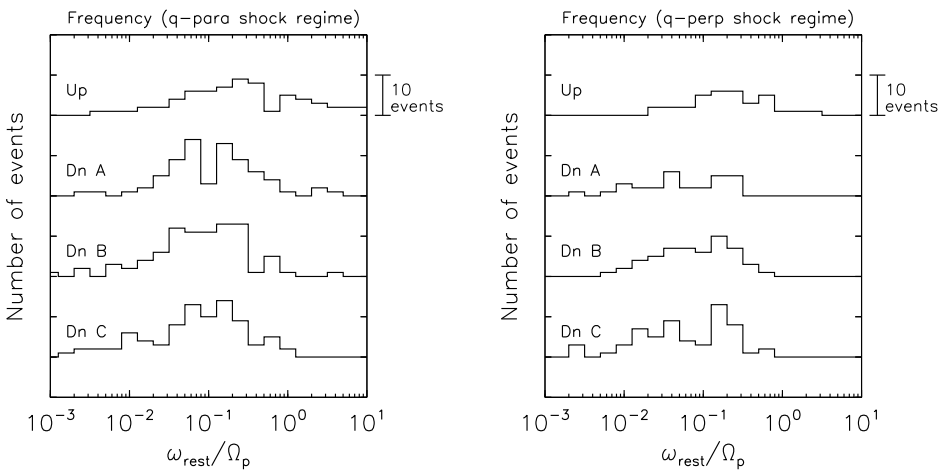

Figure 8.7: Histogram of frequencies in the plasma rest frame of reference divided by proton cyclotron frequency for the quasi-parallel shock regime (left) and for the quasiperpendicular shock regime (right). 'Up' means upstream waves. 'Dn A', 'Dn B', and 'Dn C' mean downstream waves in the outer, the middle, and the inner magnetosheath, respectively (Narita et al. 2006a).

\subsection{Results}

Distributions of the wave properties in the plasma rest frame are investigated by the above method and shown for the quasi-parallel and the quasi-perpendicular shock regimes, and from the upstream region to the inner magnetosheath region. Spatial distributions of phase velocities are also presented.

Fig. 8.7 displays histograms of the frequencies divided by the proton cyclotron frequency $\Omega_{p}$. The left panel shows the results for the quasi-parallel shock regime and the right panel for the quasi-perpendicular shock regime. Each panel shows histograms for the upstream waves (Up), the outer magnetosheath (Dn A), the middle magnetosheath (Dn B), and the inner magnetosheath (Dn C). All the histograms shown below in this section are presented in this format. The distributions peak at $\omega_{\text {rest }} / \Omega_{p} \sim 0.2-0.3$ in the quasi-parallel shock upstream region; $\omega_{\text {rest }} / \Omega_{p} \sim 0.1$ in the quasi-parallel shock downstream region; $\omega_{\text {rest }} / \Omega_{p} \sim 0.2$ in the quasi-perpendicular shock upstream region; and $\omega_{\text {rest }} / \Omega_{p} \sim 0.04-0.2$ in the quasi-perpendicular shock downstream region. The frequencies tend to be higher in the upstream region than in the downstream region for both shock regimes.

Wave numbers are compared to the one for the ion inertial length $k_{i n}=\omega_{p p} / c$ and for the ion thermal ion gyro-radius $k_{g y}=\Omega_{p} / v_{t h}$, where $\omega_{p p}$ denotes the plasma frequency for protons, $c$ the speed of light, and $v_{t h}$ the thermal velocity. Fig. 8.8 displays histograms of the wave numbers divided by $k_{i n}$. The distributions exhibit two peaks at $k / k_{i n} \sim$ 0.1 and $k / k_{\text {in }} \sim 0.4$ in the quasi-parallel shock upstream region; a persistent peak at 

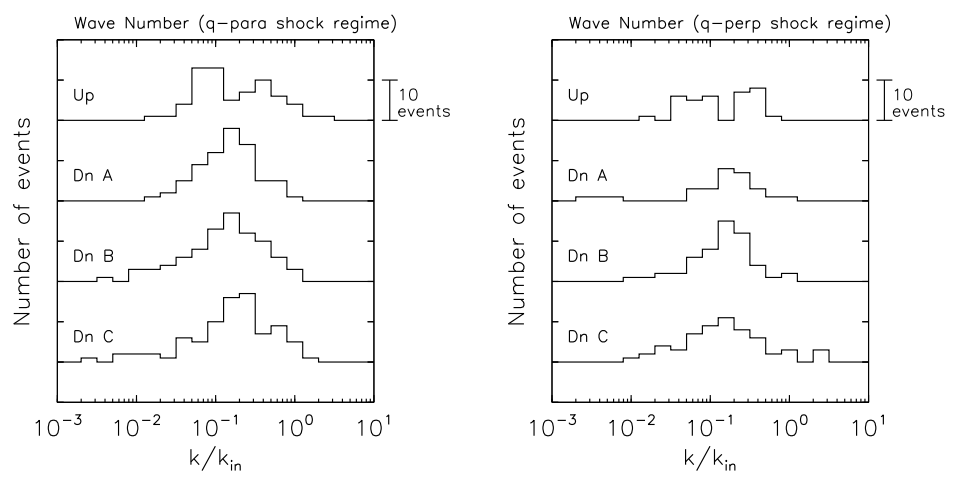

Figure 8.8: Histogram of wave numbers divided by ion inertial wave number $\omega_{p p} / c$. Panel format is the same as Fig. 8.7 (Narita et al. 2006a).

$k / k_{\text {in }} \sim 0.1-0.2$ in the quasi-parallel shock downstream region; two populations up to $k / k_{\text {in }} \sim 1$ and $k / k_{\text {in }} \sim 0.3-0.4$ in the quasi-perpendicular shock upstream region; and a persistent peak at $k / k_{i n} \sim 0.1-0.2$ in the quasi-perpendicular shock downstream region. Fig. 8.9 displays histograms of the wave numbers divided by $k_{g y}$. The distributions exhibit two peaks at $k / k_{g y} \sim 0.1$ and $k / k_{g y} \sim 0.5-0.7$ in the quasi-parallel shock upstream region; and a persistent peak at $k / k_{g y} \sim 0.2-0.6$ in the quasi-parallel shock downstream region and a peak at $k / k_{g y} \sim 1$ in the inner magnetosheath; two peaks at $k / k_{g y} \sim 0.06$ and $k / k_{g y} \sim 0.4$ in the quasi-perpendicular shock upstream region; and a peak at $k / k_{g y} \sim 0.2-0.4$ in the quasi-perpendicular shock downstream region.

Phase velocities are compared to three characteristic propagation speeds in magnetohydrodynamics: fast, intermediate, and slow mode speed. Their values are dependent on the background magnetic field, density and temperature, and propagation direction. We calculate these speeds using the background quantities given by the FGM and the CIS measurements and the propagation angle determined by the wave telescope technique. Fig. 8.10 displays histograms of the phase velocities divided by the fast mode speed $V_{f}$. The distributions exhibit peaks at $V_{p h} / V_{f} \sim 1$ in the quasi-parallel shock upstream region; $V_{p h} / V_{f} \sim 0.1-0.4$ in the quasi-parallel shock downstream region; $V_{p h} / V_{f} \sim 1$ in the quasi-perpendicular shock upstream region; and $V_{p h} / V_{f} \sim 0.1-0.7$ in the quasiperpendicular shock downstream region. Fig. 8.11 displays the ones divided by the intermediate mode speed $V_{i}$. The distributions exhibit peaks at $V_{p h} / V_{i} \sim 0.6-0.7$ and $2-3$ in the quasi-parallel shock upstream region, $V_{p h} / V_{i} \sim 1$ in the quasi-parallel shock downstream region; $V_{p h} / V_{i} \sim 1$ in the quasi-perpendicular shock upstream region; and $V_{p h} / V_{i} \sim 1$ in the quasi-perpendicular shock downstream region. Fig. 8.12 displays the ones divided by the slow mode speed $V_{s}$. Since the phase velocities of the slow mode are 

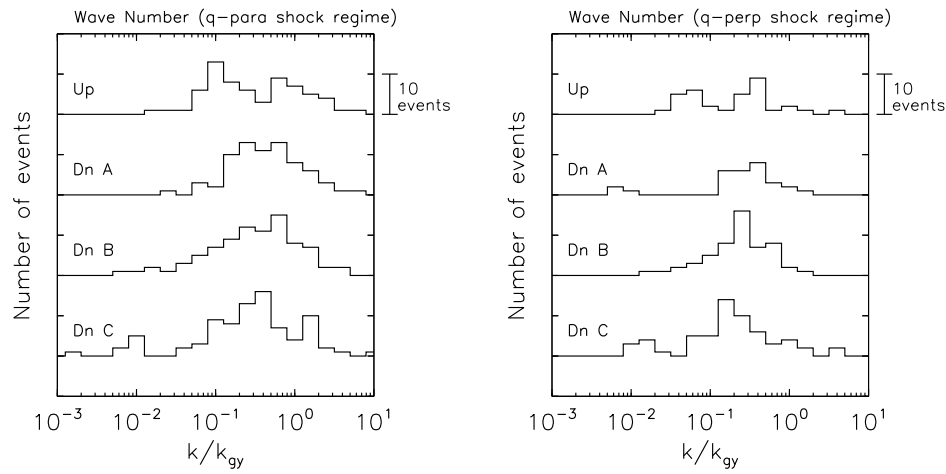

Figure 8.9: Histogram of wave numbers divided by the thermal ion gyro-wave number. Panel format is the same as Fig. 8.7 (Narita et al. 2006a).
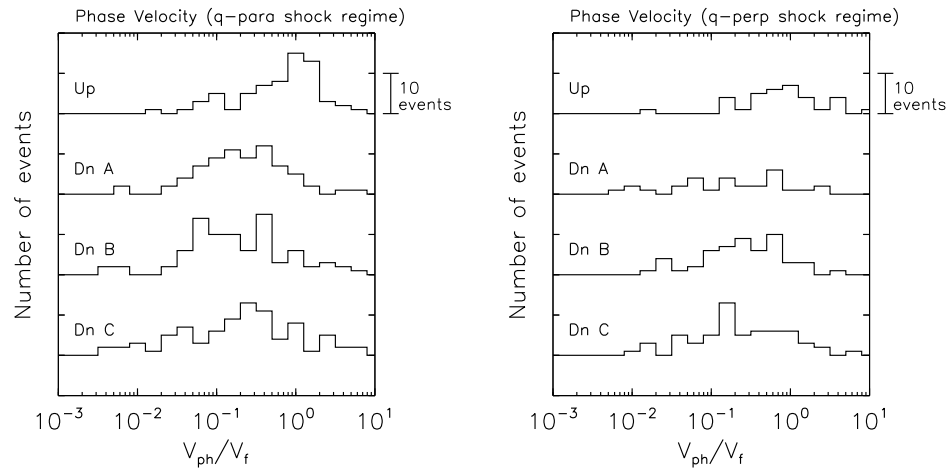

Figure 8.10: Histogram of phase velocities in the plasma rest frame divided by the one for the fast mode. Panel format is the same as Fig. 8.7 (Narita et al. 2006a).

generally close to that of the intermediate mode for the high $\beta$ plasma (e.g. Friedrichs diagram in Kivelson (1995)), the distributions exhibit similar peaks to the intermediate mode case: $V_{p h} / V_{s} \sim 2-3$ in the quasi-parallel shock upstream region; $V_{p h} / V_{s} \sim 1$ in the quasi-parallel shock downstream region; $V_{p h} / V_{s} \sim 1-2$ in the quasi-perpendicular shock upstream region; and $V_{p h} / V_{s} \sim 1$ in the quasi-perpendicular shock downstream region. The inner magnetosheath in the quasi-parallel shock regime exhibits also a peak at $V_{p h} / V_{i} \sim 3$ and $V_{p h} / V_{s} \sim 4$. 

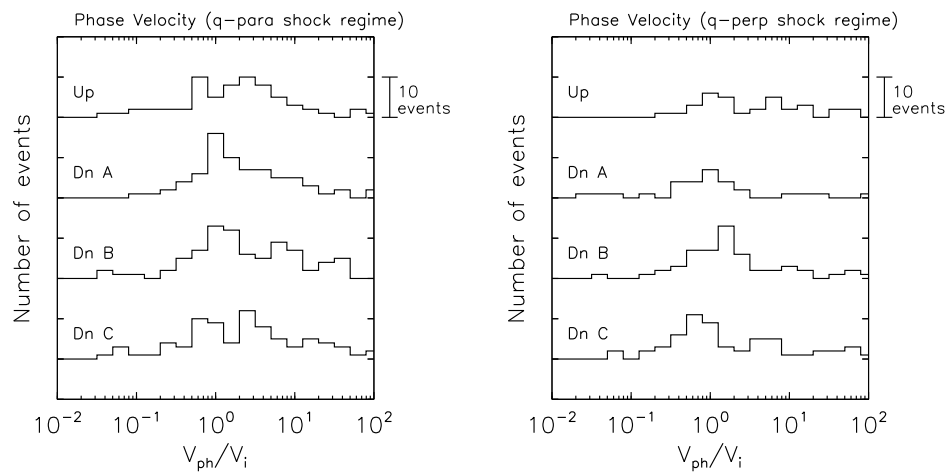

Figure 8.11: Histogram of phase velocities in the plasma rest frame divided by the one for the intermediate mode. Panel format is the same as Fig. 8.7 (Narita et al. 2006a).
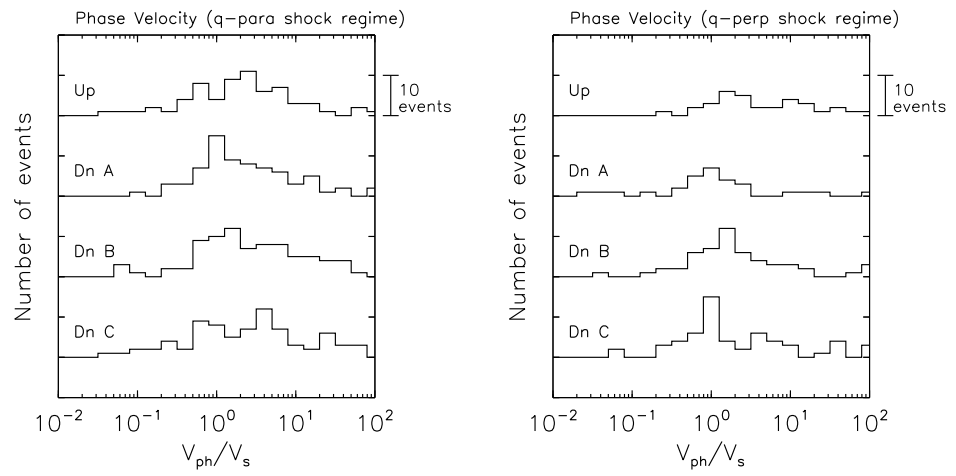

Figure 8.12: Histogram of phase velocities in the plasma rest frame divided by the one for the slow mode. Panel format is the same as Fig. 8.7 (Narita et al. 2006a).

Fig. 8.13 displays histograms of the propagation angles with respect to the background magnetic field. They are markedly different between the upstream region and the downstream region and show a similar trend in the both shock regimes. The upstream waves propagate almost parallel to the background magnetic field. In the outer magnetosheath the distribution exhibits a slight peak in the perpendicular direction. Toward the magnetopause this peak becomes clearer. The quasi-perpendicular shock upstream waves also exhibit perpendicular and anti-parallel propagation. 

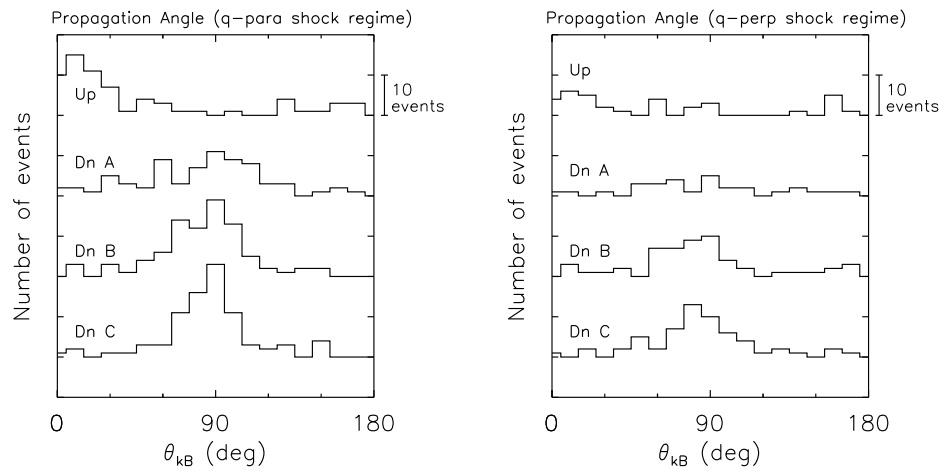

Figure 8.13: Histogram of angles between the wave vector and the background magnetic field. Panel format is the same as Fig. 8.7 (Narita et al. 2006a).
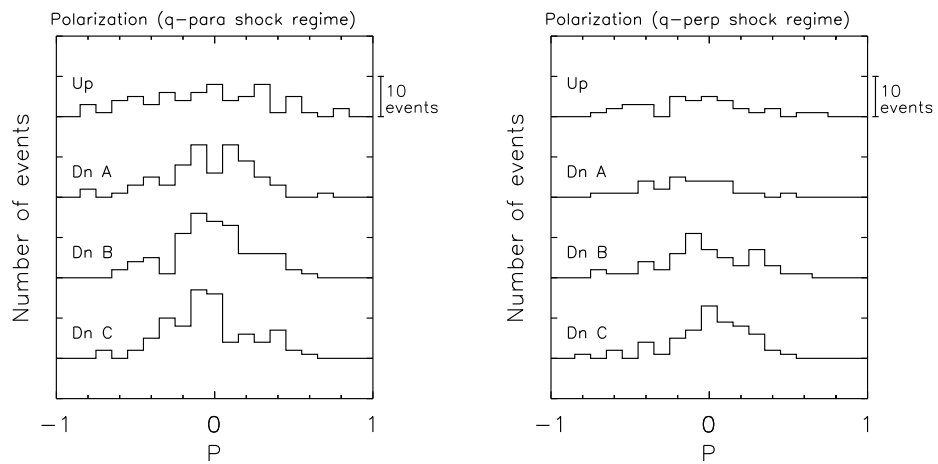

Figure 8.14: Histogram of magnetic polarization. Panel format is the same as Fig. 8.7 (Narita et al. 2006a).

Fig. 8.14 displays histograms of the magnetic polarization. In the quasi-parallel shock upstream region it $\mathrm{s}$ broadly distributed from left-hand $(p=-1)$ to right-hand polarization $(p=+1)$, while in the downstream region the linear polarization $(p=0)$ is enhanced. A similar tendency is found in the quasi-perpendicular shock regime, too.

The distributions of the phase angles between the density and the magnetic field variations $\left(\phi_{N B}\right)$ are displayed in Fig. 8.15; and the one between the plasma bulk velocity and the magnetic field variations $\left(\phi_{V B}\right)$ in Fig. 8.16. They are calculated under the condition that their respective coherence is larger than 0.7 . The quasi-parallel shock upstream 

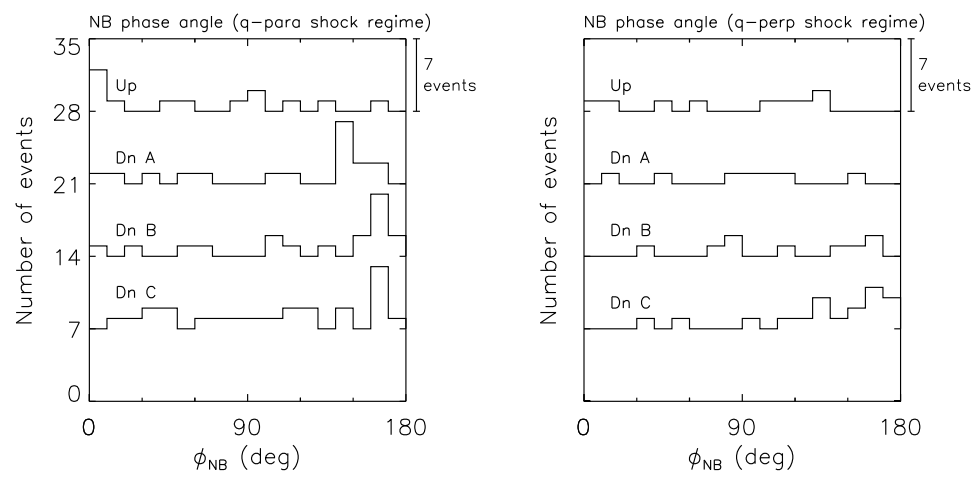

Figure 8.15: Histogram of phase angles between the density and the magnetic field strength variations. Panel format is the same as Fig. 8.7 (Narita et al. 2006a).
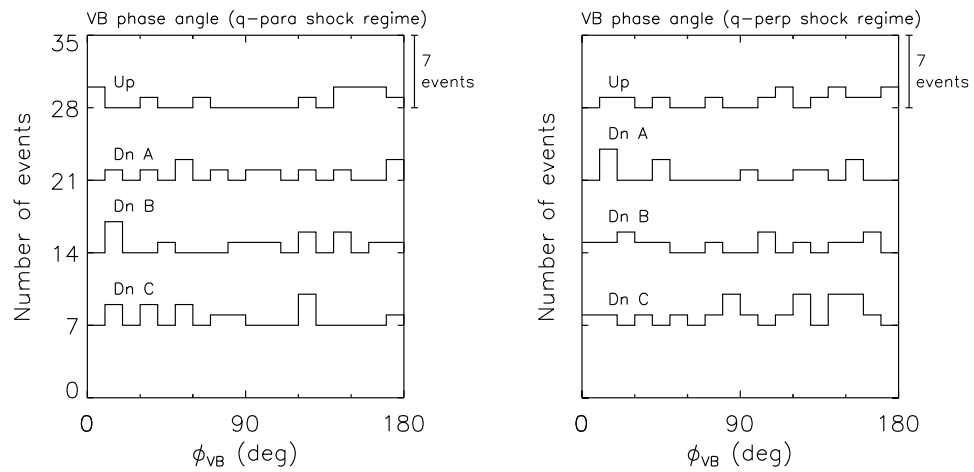

Figure 8.16: Histogram of phase angles between the plasma bulk velocity and the magnetic field variations. Panel format is the same as Fig. 8.7 (Narita et al. 2006a).

waves exhibit in-phase variations between the density and the magnetic field variations $\left(\phi_{N B} \sim 0^{\circ}\right)$ and the quasi-parallel shock downstream waves exhibit out-of-phase variations $\left(\phi_{N B} \sim 180^{\circ}\right)$. In the quasi-perpendicular shock regime only the inner magnetosheath exhibits a clear peak at $\phi_{N B} \sim 180^{\circ}$ and no clear peak is found from the upstream to the middle magnetosheath region. The histograms of $\phi_{V B}$ exhibit relatively uniform distributions in all the regions without any clear peaks.

Fig. 8.17 displays background plasma parameters: magnetic field, ion density, and 


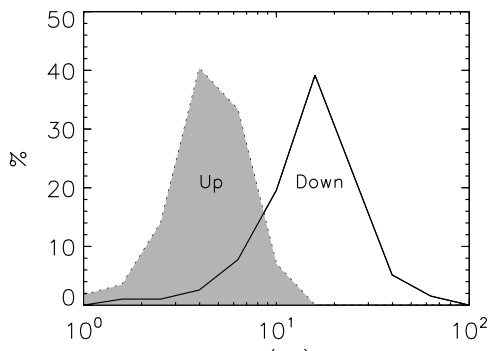

$B(n T)$
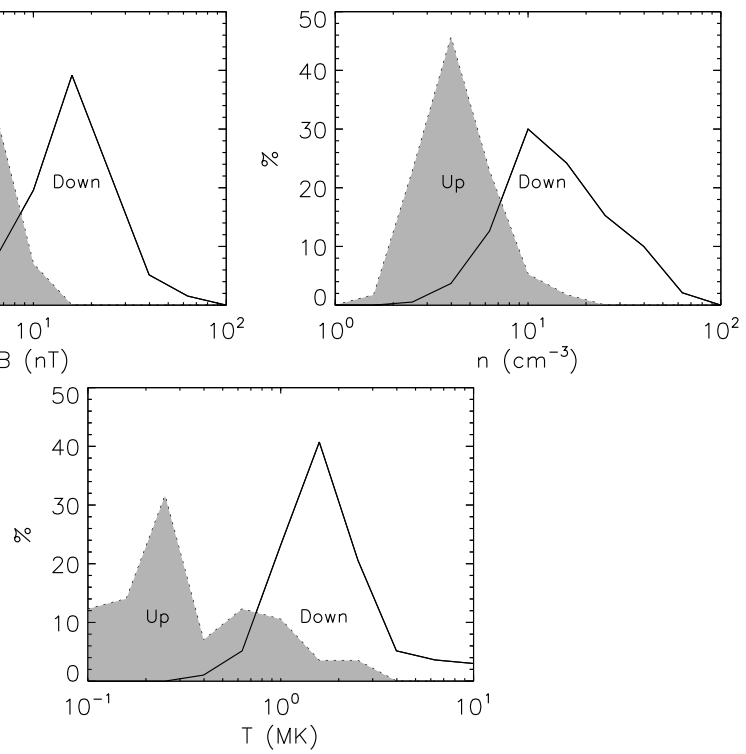

Figure 8.17: Distributions of background plasma parameters (magnetic field $B$, ion density $n$, and ion temperature $T$ ) in the upstream and the downstream regions. The unit for the $y$ axis is percentage of events with respect to the number of upstream and downstream events used for the statistical study (Narita et al. 2006a).

ion temperature. The upstream region exhibits typically the magnetic field about $4 \mathrm{nT}$, the density about $4 \mathrm{~cm}^{-3}$, and the temperature about $0.2 \times 10^{6} \mathrm{~K}$, while the downstream region exhibits the magnetic field about $15 \mathrm{nT}$, the density about $10 \mathrm{~cm}^{-3}$, and the temperature about $1.5 \times 10^{6} \mathrm{~K}$. These values yield the phase speeds about $52.5 \mathrm{~km} / \mathrm{s}$ for the fast mode, $43.6 \mathrm{~km} / \mathrm{s}$ for the intermediate mode, and also $43.6 \mathrm{~km} / \mathrm{s}$ for the slow mode for parallel propagating waves in the upstream region. In the downstream region they are $143.7 \mathrm{~km} / \mathrm{s}, 103.4 \mathrm{~km} / \mathrm{s}$, and also $103.4 \mathrm{~km} / \mathrm{s}$, respectively. Typical plasma $\beta$ is about 1.7 in the upstream region and 2.3 in the downstream region.

Comparing the two shock regimes, we find following wave characteristics in both cases: $\omega_{\text {rest }} \sim 0.2 \times \Omega_{p}, k \sim 0.1 \times k_{\text {in }}$ and $k \sim 0.4-0.5 \times k_{i n}, k \sim 0.05-0.1 \times k_{g y}$ and $k \sim 0.4-0.7 \times k_{g y}, V_{p h} \sim V_{f}$ for the upstream waves; and $\omega \sim 0.1 \times \Omega_{p}, k \sim$ $0.1-0.2 \times k_{i n}, k \sim 0.2-0.6 \times k_{g y}, V_{p h} \sim V_{i}$ and $V_{p h} \sim V_{s}$ for the downstream waves. The upstream waves propagate parallel to the magnetic field, whereas the downstream waves propagate in the perpendicular direction. The downstream waves are more linearly polarized than the upstream waves. The above properties for the magnetosheath waves are persistent from the outer to the inner magnetosheath. 


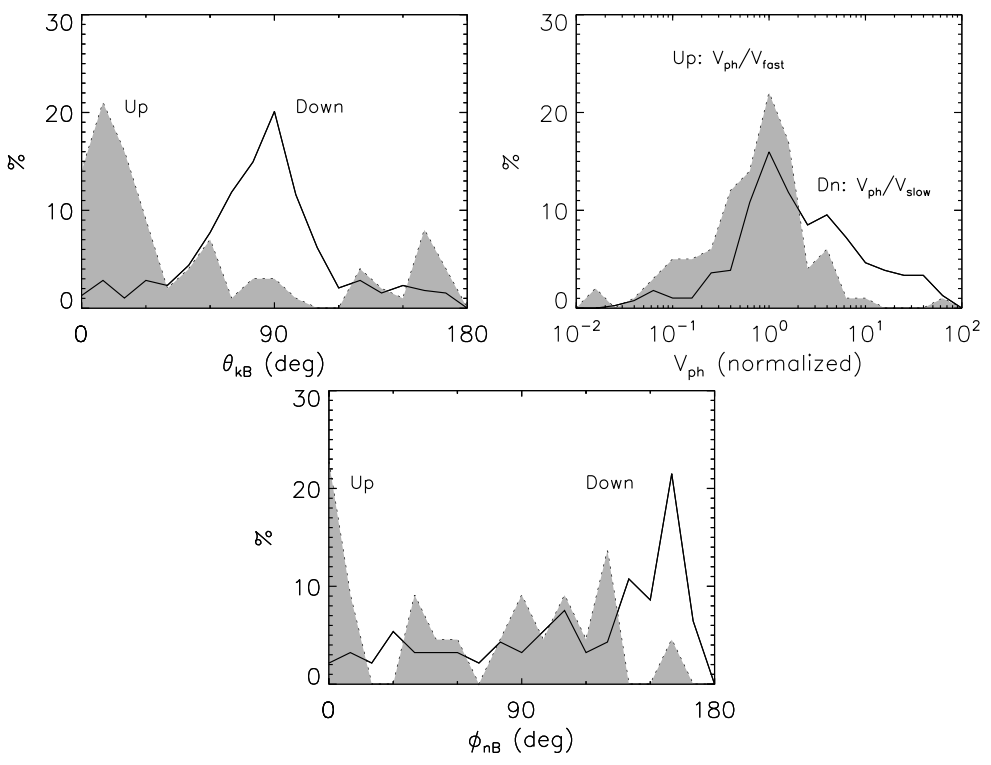

Figure 8.18: Distribution (occurrence frequency) of propagation angle, phase velocities, and phase angle between the density and the magnetic field variations for the upstream waves (filled in gray) and the downstream waves (black line). The phase velocities are divided by the fast mode speed for the upstream waves, and by the slow mode speed for the downstream waves, taking propagation angles into account (Narita and Glassmeier 2006).

Fig. 8.18 summarizes the histograms above and shows major differences of the wave properties between the upstream and the downstream region. The upstream waves are characterized by the propagation direction nearly parallel to the background magnetic field, the phase speed of the order of the fast mode speed, and the in-phase variation between the plasma density and the magnetic field, whereas The downstream waves are characterized by the perpendicular propagation, the phase speed of the order of the slow mode speed, and the out-of-phase variation (with the angle $180^{\circ}$ ) between the two quantities. Hence the upstream waves are reminiscent of the fast mode propagating nearly parallel, and the downstream waves the slow or mirror mode perpendicular.

However, the phase angle $\phi_{N B}$ shows a slight difference. In the quasi-parallel shock regime we find $\phi_{N B} \sim 0^{\circ}$ for the upstream region and $\phi_{N B} \sim 180^{\circ}$ for the downstream region, while no clear population is found for the quasi-perpendicular shock regime except for $\phi_{N B} \sim 180^{\circ}$ in the inner magnetosheath. Another difference is that the quasiperpendicular shock upstream waves exhibit oblique propagation angles. 
The distribution of the phase velocity vectors in the plasma rest frame is displayed in the $X-R$ plane $\left(R=\sqrt{Y^{2}+Z^{2}}\right)$ in the GSE system (Fig. 8.19). To aid the eyes, the original velocity vectors have been averaged over segments (shown as grids in gray). Arrows in gray and in black represent the upstream and the downstream waves, respectively. The distribution shows tendencies that the upstream waves propagate away from the shock and the downstream waves propagate toward the flank region and the magnetopause. The propagation pattern is divergent at the bow shock and convergent toward the magnetopause in the magnetosheath. The phase velocity vectors are also presented in the solar wind coordinate system in Fig. 8.20. Both the quasi-parallel shock upstream waves (arrows in gray) and the quasi-perpendicular shock upstream waves (arrows in gray-black) tend to propagate away from the shock, though some propagate toward the shock. The downstream waves near the magnetopause nose (or the $Y_{s w}=0$ line) propagate toward the magnetopause. The waves in the outer magnetosheath propagate toward the flank region aligned with the plasma flow directions, whereas they change the directions gradually toward the magnetopause in the flank region. The propagation pattern is therefore: (1) outward divergent and aligned with the shock normal direction in the upstream region; (2) away from the solar wind direction toward the flank region and aligned with the plasma flow direction in the magnetosheath; (3) inward convergent toward the magnetopause in the magnetosheath flank. This pattern is illustrated schematically in Fig. 8.21. This tendency is found in both the quasi-parallel $\left(Y_{s w}<0\right)$ and the near quasi-perpendicular shock regime $\left(Y_{s w}>0\right)$.

\subsection{Discussion}

It is of importance to estimate errors in the frequencies in the plasma rest frame, the wave number, and the phase velocity in order to verify their accuracy. For scanning in the $k$ space we use a spherical grid with $75 \times 60 \times 60$ points in radial, azimuthal, and polar direction up to Nyquist wave number $k_{N y}=3 \times 10^{-2} \mathrm{~km}^{-1}$. The uncertainty in the wave number vector is thus about $\Delta k=4 \times 10^{-4} \mathrm{~km}^{-1}$ for the magnitude and $\Delta \theta_{k B}=3^{\circ}$ for the propagation angle. The uncertainty in the rest frame frequency results from the one in the Doppler shift, $\left|\Delta \omega_{\text {rest }} / \omega_{\text {rest }}\right| \leq|\Delta k / k|+|\Delta V / V|$. The error in the phase velocity is $\left|\Delta V_{p h} / V_{p h}\right| \leq\left|\Delta \omega_{\text {rest }} / \omega_{\text {rest }}\right|+|\Delta k / k|$. Fig. 8.22 displays histograms of their respective relative errors. The error in the frequency is about $10-20 \%$, the one in the wave number is less than $10 \%$, and the one in the phase velocity is about $20 \%$.

The foreshock waves (quasi-parallel shock upstream waves) exhibit the characteristic rest frame frequencies about $0.2 \times \Omega_{p}$, which is close to the results drawn from the ISEE spacecraft observations (Hoppe et al. 1981, Hoppe and Russell 1983). The characteristic wave number is about $0.1 \times k_{i n}$ and $0.4-0.5 \times k_{i n}$, or $0.05-0.1 \times k_{g y}$ and $0.4-0.7 \times k_{g y}$. Gary et al. (1981) showed that the maximal growth rate for the right-hand ion beam instability is at about $0.1 \times k_{g y}$. Dubinin et al. (2004) also presented $k \sim 0.1 \times k_{\text {in }}$ in their oscilliton model. The results therefore confirm their conclusions but also indicate another 


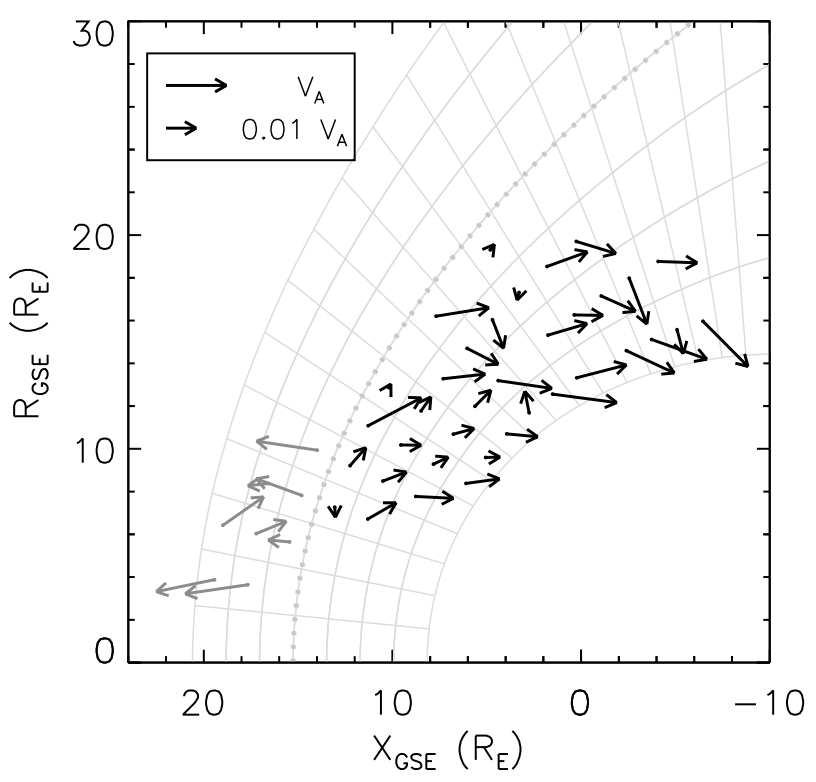

Figure 8.19: Segment averaged spatial distribution of phase velocities in the plasma rest frame in the plane of $X$ and $R=\sqrt{Y^{2}+Z^{2}}$ in the GSE coordinate system. Phase velocities normalized to the Alfvén velocity $V_{A}$ are plotted as arrows on a logarithmic scale. The arrows in gray and in black represent the upstream waves and the downstream waves, respectively. The dotted curve is the nominal bow shock calculated for the quiet solar wind condition (Narita et al. 2006a).

population. The phase velocity is of the same order as the fast mode speed in the direction nearly parallel to the background magnetic field and toward upstream, accompanied by the anti-correlated density and magnetic field variations. There are three types of ion beam instabilities known, the right-hand resonant, the left-hand resonant, and the nonresonant beam instability (Treumann and Baumjohann 1997). The right-hand and the lefthand mode are essentially the magnetosonic/whistler mode and the Alfvén/ion cyclotron mode, respectively (Gary 1986). The results prefer that the foreshock waves represent the waves driven by the right-hand mode instability, judging from the wave numbers, the phase velocities, and the transport ratios. However, not only the right-hand but also the linear and the left-hand polarization about the magnetic field are detected. Perhaps the left-hand mode is also excited in the foreshock, as suggested by Eastwood et al. (2003). 


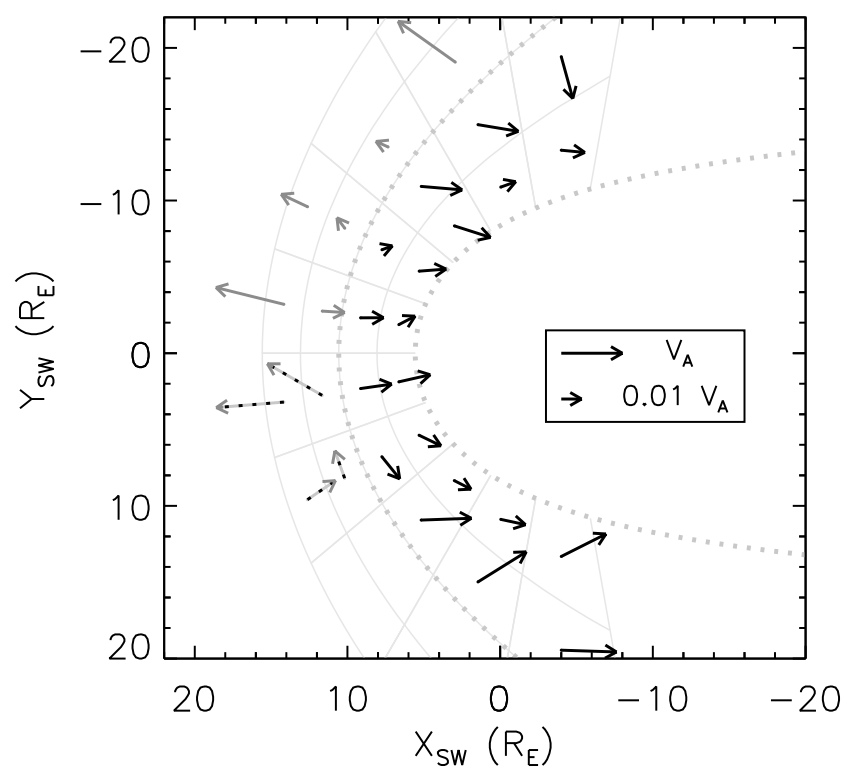

Figure 8.20: Segment averaged spatial distribution of phase velocities in the plasma rest frame in the plane of $X$ and $Y$ in the solar wind coordinate system. The arrows in gray, in gray-black, and in black represent the foreshock waves, the quasi-perpendicular shock upstream waves and the magnetosheath waves, respectively (Narita et al. 2006a).

The non-resonant mode propagates in the opposite direction to the ion beam, i.e. in the anti-sunward direction. Some waves are identified to propagate in this direction, as seen in Fig. 8.19 and Fig 8.20. The majority of the foreshock waves are the right-hand mode, but we do not exclude the possibility of the left-hand and the non-resonant mode.

Waves upstream of the quasi-perpendicular shock exhibit similar properties to the foreshock waves. The quasi-perpendicular shock is accompanied by the foot region whose length is of the order of the ion convective gyroradius, $V_{s w} / \Omega_{p}$ (the solar wind velocity divided by the upstream proton gyrofrequency). Under the typical solar wind conditions it is a few hundred $\mathrm{km}$, whereas the waves upstream of the quasi-perpendicular shock used in our investigation are found at about $30,000 \mathrm{~km}$ from the shock. Therefore the detected waves can not simply be called the foot waves. The waves exhibit typically rest frame frequencies at about $0.2 \times \Omega_{p}$ and wave numbers at about $0.1 \times k_{i n}$ and $0.4-$ $0.5 \times k_{i n}$, or $0.06 \times k_{g y}$ and $0.4 \times k_{g y}$, which result in that they propagate primarily 


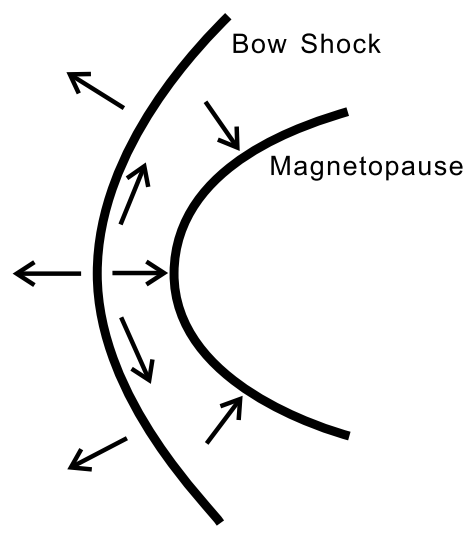

Figure 8.21: Simplified sketch of the propagation pattern. Arrows represent wave phase velocities. The outer and inner solid curved lines represent the bow shock and the magnetopause, respectively (Narita et al. 2006a).

at phase speeds close to the fast mode speed. Unlike the quasi-parallel shock case, the phase angle $\phi_{N B}$ is not clear, which makes it difficult to interpret them simply as the fast mode. The waves detected in this region may be either the waves in the solar wind or the ones from the shock, or a mixture of them. While the Alfvén waves propagating in the anti-sunward direction may be dominant in the former case (Velli and Pruneti 1997, Biskamp 2003), the whistler waves parallel to oblique to the background magnetic field are often detected in the foot region which may escape into the solar wind in the latter case. Fairfield (1974), Balikhin et al. (1997a) suggested that the whistler wave upstream of the quasi-perpendicular shock is typically of the order of $100 \mathrm{~km}$, but the detected waves have wavelengths typically at about $6000 \mathrm{~km}$. The wave mode identification in this region needs dispersion analysis in future.

In the magnetosheath the wave properties are reminiscent of the mirror modes, as the wave vectors are predominantly perpendicular to the background magnetic field, and the magnetic field fluctuations are linearly polarized and anti-correlated to that of the density. The characteristic wave number is about $0.1-0.2 \times k_{i n}$ which is slightly smaller than the result drawn by the linear Vlasov theory, $0.3-0.5 \times k_{i n}$ (Gary 1992). The origin of this difference should be studied more. It is interesting that the frequency is about $0.1 \times \Omega_{p}$, which is comparable to the one of the upstream waves. The finite frequencies result in the finite phase velocities that are of the same order as the intermediate mode or the slow mode speed. Therefore it is still possible to interpret the magnetosheath waves as the intermediate or the slow mode waves in terms of the phase velocities. However, the transport ratio $\phi_{V B}$ shows scattered distributions and disagrees with the intermediate mode interpretation. The slow mode waves should be heavily damped in kinetic theory 

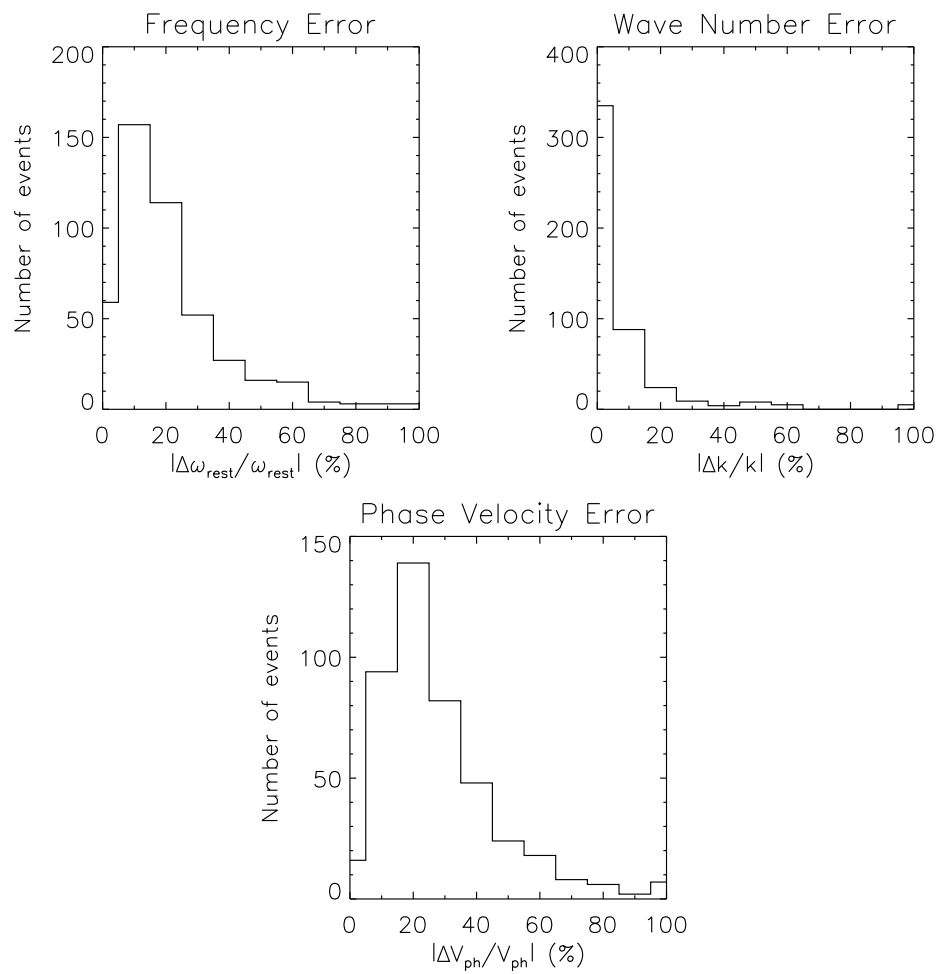

Figure 8.22: Histograms of relative errors in the frequencies, in the wave numbers, and in the phase velocities (Narita et al. 2006a).

under magnetosheath conditions (Schwartz et al. 1996). In addition both modes exhibit group velocity directions guided by the background magnetic field. The finite frequencies and propagation speeds may be due to a variety of processes. Stasiewicz (2004a,b) argued that nonlinear effects in the fluid model allow slow mode solitons to exist, which represent propagating mirror modes. But we note that the nonlinear effects may explain the amplitude and phase velocities but they do not explain the reason why the waves prefer perpendicular propagation or how they are generated. Gradients of the background density coupled to the mirror modes, called the drift mirror modes, are also a possible interpretation (Hasegawa 1969, Pokhotelov et al. 2001). Early spacecraft observations have already led to this interpretation (Tsurutani et al. 1982, Hubert et al. 1989, Anderson et al. 1994, Denton et al. 1995). Or more generally, not only the density gradients but also the temperature gradients which are imposed by the magnetopause as a boundary may 
be coupled to the mirror modes so that they acquire a finite frequency by means of diamagnetic drift effect, as Johnson and Cheng (1997) present the idea of the global mirror modes in the magnetosheath. Identifying the excitation and propagation mechanism of the magnetosheath waves is an interesting problem, and the multi-point measurements of Cluster would be helpful for solving it, for example, investigating the dispersion curves and group velocities, and comparing the rest frame frequencies with the drift mirror mode frequency or the diamagnetic drift frequency.

We also find that the wave propagation is away from the shock in both the upstream and the downstream regions. Matsuoka et al. (2000) and Schäfer et al. (2005) derived that the propagation sense in the magnetosheath is anti-sunward and outward in the same direction as the plasma flow for the Alfvénic fluctuations of the magnetic field. The results confirm their conclusion and in addition indicate that it is toward the magnetopause in the magnetosheath flank. Song et al. (1994) present that the waves are phase standing in the flow near the magnetopause and therefore have a phase velocity oriented toward the shock in the plasma rest frame. Omidi and Winske (1995) interpret that such waves are the mirror modes that pile up at the magnetopause boundary, but the study does not derive the magnetosheath waves propagating toward the shock. Perhaps such waves are found so close to the magnetopause that the event selection procedure does not count them.

We do not obtain clear signals for the ion cyclotron waves that may also be expected to appear in the magnetosheath. Although the phase velocities detected are of the order of the intermediate mode speed, they are also of the same order as the slow mode speed and furthermore the propagation angles are predominantly in the perpendicular direction to the background magnetic field. There is no clear difference in the magnetosheath between the quasi-parallel and the quasi-perpendicular shock regime for most of wave properties such as frequencies, wave numbers and so on, but the phase angle $\phi_{N B}$ is not clear in the outer and the middle magnetosheath in the quasi-perpendicular shock regime.

Krauss-Varban and Omidi (1991, 1993) presented numerical simulations on the waves upstream and downstream of the quasi-parallel shock and showed that the upstream fast mode waves are mode converted into the downstream ion cyclotron waves across the shock, while the wave vectors are oriented toward upstream in both cases. But the results show that the wave vectors are oriented in the opposite direction to their conclusion in the downstream region. The wave properties are not succeeded or transmitted from the upstream region to the downstream region as they argue.

\subsection{Summary}

In this chapter we have addressed the question of what typical wave properties in the upstream and the downstream region of the terrestrial bow shock are, how these waves propagate, and how their characteristics differ between the quasi-parallel and the quasiperpendicular shock regimes. The data used for the analysis are magnetic field and plasma measurements made on board the four Cluster spacecraft. Cross spectral analyses as well 
as the wave telescope technique have been used to analyze the observed wave activity. As the analysis allows to determine the Doppler shift, all results have been presented in the plasma rest frame. As for most data intervals analyzed it is determined whether observations are made in the quasi-parallel or the quasi-perpendicular regime. An approximate classification was made by introducing a special solar wind coordinate system which provides a fast classification based on the ACE solar wind and magnetic field data. As we are also interested in knowing whether wave properties depend on where in the magnetosheath the waves are observed, their properties are classified according to observations made into the upstream region, the outer, the middle, and the inner magnetosheath.

Moving from the upstream region through the shock toward the magnetopause, propagation directions change from being parallel to perpendicular to the background magnetic field. In the quasi-parallel shock regime this change is accompanied by a change of the transport ratios of the phase angles between the magnetic field and the density fluctuations from in-phase variations to out-of-phase variations, that is the upstream waves exhibit the fast mode characteristics and the downstream waves the mirror or the slow mode characteristics. Phase velocities agree with the fast mode speed in the upstream region and the slow mode speed in the downstream region, respectively. In the quasi-perpendicular shock regime the transport ratio does not show clear signals except for the out-of-phase variation in the inner magnetosheath. But otherwise the both regimes exhibit similar properties in frequency, wave number, phase velocity, propagation angle, and polarization in both the upstream and the downstream region. In the magnetosheath there is no clear difference in these properties among the outer, the middle, and the inner magnetosheath.

The spatial pattern of wave propagation directions indicates an interesting structure. Propagation is outward divergent in the upstream region, inward divergent in the magnetosheath near the magnetopause nose, and inward convergent in the magnetosheath flank. The divergent pattern in the magnetosheath indicates that the waves propagate along the plasma stream lines, following the refraction of the plasma flow at the shock. The convergent pattern in the magnetosheath flank which is consistent with the perpendicular propagation in the draped magnetic field.

We conclude that wave properties are different the upstream and downstream region, but they are similar between the quasi-parallel and the quasi-perpendicular shock regime, suggesting that the upstream waves are not transmitted to the downstream region across the shock and that the downstream waves do not depend on the shock angle. Our results also suggest that in the foreshock the ion beam instabilities are operating with dominant right-hand mode and possibly with less dominant left-hand and non-resonant mode being excited. Upstream of the quasi-perpendicular shock the wave properties are similar to that of the foreshock waves, but further investigations are needed to identify the waves modes. Both slow mode and mirror mode interpretations are possible for the magnetosheath waves. In any case the magnetosheath waves have finite frequencies and phase velocities that could result from various effects such as nonlinear effects and inhomogeneities. 
Table 8.1: Time intervals used in the statistical study of the magnetosheath waves, observation Configurations of the magnetosheath observations (near quasi-parallel or near quasi-perpendicular shock regime; magneothsheath shell), and dominant frequencies in the spacecraft frame taken from 4-s and 1-s data set. A, B and C used in the magnetosheath shell stand for the outer, the middle, and the inner magnetosheath, respectively

\begin{tabular}{ccccc}
\hline Interval & shock & shell & $\begin{array}{c}f_{s c(l o w)} \\
\mathrm{mHz}\end{array}$ & $\begin{array}{c}f_{s c(h i g h)} \\
\mathrm{mHz}\end{array}$ \\
\hline 2002-02-03 / 06:00-06:35 & q-para & $\mathrm{A}$ & 56.6 & 187.5 \\
2002-02-03 / 07:10-07:45 & q-para & $\mathrm{B}$ & 40.0 & 125.0 \\
2002-02-03 / 08:25-09:00 & q-para & $\mathrm{C}$ & 41.0 & 89.8 \\
2002-02-04 / 08:00-08:35 & q-perp & $\mathrm{A}$ & 77.1 & 191.4 \\
2002-02-04 / 03:45-04:20 & q-perp & $\mathrm{B}$ & 110.4 & 203.1 \\
2002-02-04 / 01:40-02:15 & q-perp & $\mathrm{C}$ & 10.7 & 238.3 \\
2002-02-06 / 11:55-12:30 & q-perp & $\mathrm{A}$ & 52.7 & 105.5 \\
2002-02-06 / 10:30-11:05 & q-perp & $\mathrm{B}$ & 34.2 & 179.7 \\
2002-02-08 / 23:20-23:55 & q-para & $\mathrm{A}$ & 43.9 & 191.4 \\
2002-02-08 / 21:00-21:35 & q-perp & $\mathrm{B}$ & 10.7 & 218.8 \\
2002-02-08 / 18:30-19:05 & q-perp & $\mathrm{C}$ & 35.2 & 136.7 \\
2002-02-11 / 09:00-09:35 & q-perp & $\mathrm{A}$ & 59.6 & 246.1 \\
2002-02-11 / 06:30-07:05 & q-perp & $\mathrm{B}$ & 42.0 & 195.3 \\
2002-02-11 / 02:30-03:05 & q-perp & $\mathrm{C}$ & 24.4 & 187.5 \\
2002-02-12 / 16:30-17:05 & q-para & $\mathrm{A}$ & 91.8 & 191.4 \\
2002-02-12 / 18:30-19:05 & q-para & $\mathrm{B}$ & 12.7 & 238.3 \\
2002-02-12 / 20:30-21:05 & q-para & $\mathrm{C}$ & 74.2 & 265.6 \\
2002-02-13 / 13:30-14:05 & q-perp & $\mathrm{B}$ & 27.3 & 191.4 \\
2002-02-15 / 01:00-01:35 & q-para & $\mathrm{A}$ & 42.0 & 140.6 \\
2002-02-15 / 03:00-03:35 & q-perp & $\mathrm{B}$ & 45.9 & 289.1 \\
2002-02-15 / 04:30-05:05 & q-perp & $\mathrm{C}$ & 50.8 & 175.8 \\
2002-02-16 / 05:00-05:35 & q-perp & $\mathrm{A}$ & 44.9 & 171.9 \\
2002-02-16 / 02:30-03:05 & q-para & $\mathrm{B}$ & 53.7 & 164.1 \\
2002-02-16 / 00:00-00:35 & q-para & $\mathrm{C}$ & 66.4 & 82.0 \\
2002-02-18 / 07:00-07:35 & q-perp & $\mathrm{A}$ & 74.2 & 175.8 \\
2002-02-18 / 06:10-06:45 & q-para & $\mathrm{B}$ & 95.7 & 156.2 \\
2002-02-18 / 05:10-05:45 & q-para & $\mathrm{C}$ & 66.4 & 238.3 \\
2002-02-19 / 17:30-18:05 & q-para & $\mathrm{A}$ & 41.0 & 171.9 \\
2002-02-19 / 20:30-21:05 & q-para & $\mathrm{B}$ & 60.5 & 265.6 \\
2002-02-19 / 23:00-23:35 & q-para & $\mathrm{C}$ & 83.0 & 296.9
\end{tabular}




\begin{tabular}{|c|c|c|c|c|}
\hline 200 & q-para & A & 83.0 & .2 \\
\hline $002-02-20 / 14: 30-15: 05$ & q-para & $\mathrm{B}$ & 36.1 & 39.1 \\
\hline $002-02-20 / 13: 15-13: 50$ & q-para & $\mathrm{C}$ & 34.2 & 144.5 \\
\hline 2002-02-23 / 05:30-06:05 & q-perp & A & 43.0 & 261.7 \\
\hline 2002-02-23 / 02:00-02:35 & q-para & $\mathrm{B}$ & 114.3 & 136.7 \\
\hline $002-02-22$ / 23:00-23:35 & q-perp & $\mathrm{C}$ & 48.8 & 210.9 \\
\hline 2002-02-25 / 08:45-09:20 & q-perp & $\mathrm{B}$ & 24.4 & 43.0 \\
\hline 2002-02-25 / 08:00-08:35 & q-perp & $\mathrm{C}$ & 39.1 & 242.2 \\
\hline $2002-02-26 / 22: 15-22: 50$ & q-para & A & 72.3 & 121.1 \\
\hline 2002-02-26 / 23:15-23:50 & q-perp & $\mathrm{B}$ & 50.8 & 128.9 \\
\hline 2002-02-27 / 00:15-00:50 & q-para & $\mathrm{C}$ & 35.2 & 199.2 \\
\hline $2002-02-27$ / 23:00-23:35 & q-para & A & 53.7 & 175.8 \\
\hline 2002-02-27 / 19:30-20:05 & q-perp & $\mathrm{B}$ & 34.2 & 261.7 \\
\hline 2002-02-27 / 16:30-17:05 & q-perp & $\mathrm{C}$ & 34.2 & 160.2 \\
\hline 2002-03-01 / 09:00-09:35 & q-perp & A & 66.4 & 218.8 \\
\hline 2002-03-02 / 07:45-08:20 & q-para & A & 36.1 & 175.8 \\
\hline $2002-03-02 /($ & q-para & $\mathrm{B}$ & 28.3 & 281.2 \\
\hline 2002-03-02 / 04:00-04:35 & q-para & $\mathrm{C}$ & 10.7 & 187.5 \\
\hline 2002-03-03 / 18:00-18:35 & q-para & A & 51.8 & 269.5 \\
\hline 2002-03-03 / 20:00-20:35 & q-para & $\mathrm{B}$ & 53.7 & 293.0 \\
\hline 2002-03-03 / 21:25-22:00 & q-para & $\mathrm{C}$ & 14.6 & 62.5 \\
\hline 2002-03-04 / 13 & q-perp & A & 114.3 & 43.0 \\
\hline 2002-03-04 / 11:40-12:15 & q-perp & $\mathrm{B}$ & 46.9 & 269.5 \\
\hline 2002-03-04 / 10:00-10:35 & q-perp & $\mathrm{C}$ & 74.2 & 78.1 \\
\hline 2002-03-06 / 02:15-02:50 & q-para & A & 78.1 & 277.3 \\
\hline 2002-03-06 / 03:15-03:50 & q-para & $\mathrm{B}$ & 45.9 & 261.7 \\
\hline 2002-03-09 / 09:55-10:30 & q-para & A & 31.2 & 332.0 \\
\hline 2002-03-09 / 08:30-09:05 & q-perp & $\mathrm{B}$ & 102.5 & 222.7 \\
\hline 2002-03-09 / 06:45-07:20 & q-para & $\mathrm{C}$ & 70.3 & 293.0 \\
\hline 2002-03-10 / 19:00-19:35 & q-para & A & 66.4 & 183.6 \\
\hline $2002-03-10 / 21: 30-22: 05$ & q-perp & $\mathrm{B}$ & 64.5 & 187.5 \\
\hline 2002-03-10 / 23:20-23:55 & q-perp & $\mathrm{C}$ & 43.0 & 230.5 \\
\hline 2002-03-11 / 18:00-18:35 & q-perp & A & 65.4 & 289.1 \\
\hline 2002-03-11 / 16:00-16:35 & q-perp & $\mathrm{B}$ & 75.2 & 199.2 \\
\hline 2002-03-11/ 14:00-14:35 & q-perp & $\mathrm{C}$ & 16.6 & 328.1 \\
\hline 2002-03-13 / 05:45-06:20 & q-para & A & 30.3 & 128.9 \\
\hline 2002-03-13 / 07:15-07:50 & q-perp & $\mathrm{B}$ & 37.1 & 132.8 \\
\hline 2002-03-13 / 09:15-09:50 & q-para & $\mathrm{C}$ & 52.7 & 207.0 \\
\hline
\end{tabular}




\begin{tabular}{|c|c|c|c|c|}
\hline 05 & q-perp & A & 24.4 & \\
\hline 2002-03-14 / 03:00-03:35 & q-perp & $\mathrm{B}$ & 22.5 & 2656 \\
\hline 02-03-14 / 01:30-02:05 & perp & $\mathrm{C}$ & 30.3 & 242.2 \\
\hline 2002-03-16 / 10:45-11:20 & q-para & A & 114.3 & 187.5 \\
\hline $2002-03-1$ & q-perp & $\mathrm{B}$ & & \\
\hline 7:05 & q-perp & $\mathrm{C}$ & 46.9 & 7.0 \\
\hline 5-17:10 & q-perp & A & 10.7 & \\
\hline 2002 & q-perp & $\mathrm{B}$ & 112.3 & 218.8 \\
\hline 2002-03 & q-perp & $\mathrm{C}$ & 31.2 & 0.0 \\
\hline $2002-03-23 / 13$ & q-para & A & 65.4 & 101.6 \\
\hline 35 & q-para & $\mathrm{B}$ & 12.7 & \\
\hline 35 & & $\mathrm{C}$ & 72.3 & 6.2 \\
\hline 2002 & q-perp & $\mathrm{B}$ & 96.7 & 35.9 \\
\hline 2002-03-25 / 06: & q-perp & $\mathrm{C}$ & 50.8 & 398.4 \\
\hline 2002 & q-para & A & 63.5 & 125.0 \\
\hline 35 & q-perp & $\mathrm{B}$ & 92.8 & 8.9 \\
\hline 200 & q-para & A & 4.3 & \\
\hline $16: 35$ & $q-p$ & $\mathrm{~B}$ & 36.1 & 191.4 \\
\hline 2002-03-30 / 1 & q-para & $\mathrm{C}$ & 31.2 & 210.9 \\
\hline $2002-0$ & q-para & A & 114.3 & 238.3 \\
\hline $2002-($ & q-para & $\mathrm{B}$ & 54.7 & 109.4 \\
\hline 2002 & q-perp & C & & \\
\hline $2002-($ & q-perp & B & 26.4 & 152.3 \\
\hline 2002-04- & q-para & $\mathrm{C}$ & 33.2 & 164.1 \\
\hline 2002-04-04 / 1 & q-para & A & 60.5 & 171.9 \\
\hline $2002-04-0$ & q-para & $\mathrm{B}$ & & 8.3 \\
\hline 2002-04-04 / 10: & q-para & $\mathrm{C}$ & 26.4 & 168.0 \\
\hline 2002 & q-perp & A & 47.9 & 269.5 \\
\hline 2002-04-05 / 21:25-22:00 & q-perp & $\mathrm{B}$ & 61.5 & 293.0 \\
\hline $2002-04-05 / 22: 25-23: 00$ & q-perp & $\mathrm{C}$ & 57.6 & 210.9 \\
\hline 2002-04-07 / 00:30-01:05 & q-para & A & 49.8 & 257.8 \\
\hline $2002-04-06 / 22: 00-22: 35$ & q-para & $\mathrm{B}$ & 112.3 & 195.3 \\
\hline 2002-04-06 / 19:15-19:50 & q-para & $\mathrm{C}$ & 39.1 & 199.2 \\
\hline 2002-04-08 / 05:00-05:35 & q-para & $\mathrm{A}$ & 41.0 & 335.9 \\
\hline 2002-04-08 / 06:10-06:45 & q-para & B & 37.1 & 175.8 \\
\hline 2002-04-09 / 07:10-07:45 & q-pe & $\mathrm{A}$ & 55.7 & 238.3 \\
\hline 2002-04-09 / 06:15-06:50 & $\mathrm{q}-\mathrm{p}$ & $\mathrm{B}$ & 52.7 & 214.8 \\
\hline 2002-04-09 / 05:10-05:45 & q-para & $\mathrm{C}$ & 56.6 & 308 \\
\hline
\end{tabular}




\begin{tabular}{|c|c|c|c|c|}
\hline 200 & q-para & A & 48.8 & 140.6 \\
\hline $002-04-10 / 20: 00-20: 35$ & q-para & $\mathrm{B}$ & 31.2 & 125.0 \\
\hline $002-04-10 / 22: 10-22: 45$ & q-para & $\mathrm{C}$ & 27.3 & 246.1 \\
\hline 2002-04-13 / 03:00-03:35 & q-para & A & 85.9 & 109.4 \\
\hline 2002-04-13 / 04:45-05:20 & q-para & $\mathrm{B}$ & 31.2 & 199.2 \\
\hline $002-04-13$ / 06:10-06:45 & q-para & $\mathrm{C}$ & 10.7 & 199.2 \\
\hline 2002-04-14 / 00:00-00:35 & q-para & A & 44.9 & 148.4 \\
\hline 2002-04-13 / 22:00-22:35 & q-para & $\mathrm{B}$ & 100.6 & 234.4 \\
\hline 2002-04-13 / 20:15-20:50 & q-perp & $\mathrm{C}$ & 58.6 & 199.2 \\
\hline 2002-04-15 / 14:15-14:50 & q-perp & $\mathrm{B}$ & 32.2 & 144.5 \\
\hline 2002-04-15 / 15:40-16:15 & q-perp & $\mathrm{C}$ & 25.4 & 433.6 \\
\hline 2002-04-18 / 22:00-22:35 & q-para & $\mathrm{B}$ & 101.6 & 105.5 \\
\hline 2002-04-18 / 19:00-19:35 & q-para & $\mathrm{C}$ & 107.4 & 39.1 \\
\hline 2002-04-21 / 07:00-07:35 & q-para & $\mathrm{B}$ & 21.5 & 101.6 \\
\hline 2002-04-21 / 04:30-05:05 & q-para & $\mathrm{C}$ & 38.1 & 121.1 \\
\hline 2002-04-22 / 14:00-14:35 & q-para & $\mathrm{B}$ & 16.6 & 39.1 \\
\hline 2002-04-22 / 16:30-17:05 & q-para & $\mathrm{C}$ & 111.3 & 132.8 \\
\hline $2002-04-23 / 12: 15-12: 50$ & q-para & A & 47.9 & 195.3 \\
\hline 2002-04-23 / 10:30-11:05 & q-perp & $\mathrm{B}$ & 77.1 & 156.2 \\
\hline 2002-04-23 / 08:45-09:20 & q-para & $\mathrm{C}$ & 64.5 & 50.8 \\
\hline 2002-04-24 / 18:10-18:45 & q-perp & A & 10.7 & 277.3 \\
\hline 2002-04-24 / 19:40-20:15 & q-para & $\mathrm{B}$ & & 261.7 \\
\hline 2002-04-26 / 01:00-01:35 & q-para & $\mathrm{C}$ & 60.5 & 234.4 \\
\hline 2002-04-28 / 07:10-07:45 & q-para & A & 39.1 & 183.6 \\
\hline 2002-04-28 / 06:00-06:35 & q-para & $\mathrm{B}$ & 72.3 & 242.2 \\
\hline 2002-04-30 / 19:30-20:05 & q-para & A & 114.3 & 113.3 \\
\hline 2002-04-30 / 15:30-16:05 & q-perp & $\mathrm{B}$ & 33.2 & 218.8 \\
\hline 2002-04-30 / 13:30-14:05 & q-para & $\mathrm{C}$ & 57.6 & 78.1 \\
\hline 2002-05-03 / 06:30-07:05 & q-para & A & 45.9 & 281.2 \\
\hline 2002-05-03 / 04:00-04:35 & q-para & $\mathrm{B}$ & 37.1 & 285.2 \\
\hline 2002-05-03 / 02:00-02:35 & q-para & $\mathrm{C}$ & 38.1 & 140.6 \\
\hline 2002-05-05 / 10:30-11:05 & q-para & A & 39.1 & 117.2 \\
\hline 2002-05-05 / 09:00-09:35 & q-para & $\mathrm{B}$ & 43.9 & 144.5 \\
\hline 2002-05-05 / 07:30-08:05 & q-para & $\mathrm{C}$ & 27.3 & 105.5 \\
\hline $2002-05-06 / 21: 00-21: 35$ & q-para & $\mathrm{B}$ & 24.4 & 281.2 \\
\hline 2002-05-06 / 23:15-23:50 & q-perp & $\mathrm{C}$ & 15.6 & 105.5 \\
\hline 2002-05-07 / 22:20-22:55 & q-para & A & 78.1 & 160.2 \\
\hline 2002-05-07 / 20:00-20:35 & $\mathrm{q}-1$ & $\mathrm{~B}$ & 104.5 & 156.2 \\
\hline
\end{tabular}




\begin{tabular}{|c|c|c|c|c|}
\hline 17 / 17 & q-para & $\mathrm{C}$ & 79 & \\
\hline 02-05-09 / 06:25-07:00 & q-perp & $\mathrm{C}$ & 111.3 & 2344 \\
\hline $10 / 07: 15-07: 50$ & perp & $\mathrm{A}$ & 5.2 & 69.5 \\
\hline $02-05-10 / 06: 15-06: 50$ & q-perp & $\mathrm{B}$ & 33.2 & 214.8 \\
\hline :35 & $\mathrm{rp}$ & $\mathrm{C}$ & 9 & 97.7 \\
\hline 35 & q-para & $\mathrm{B}$ & 38.1 & 222.7 \\
\hline 35 & q-para & $\mathrm{C}$ & 11.7 & \\
\hline :35 & -perp & $\mathrm{B}$ & 85.9 & 269.5 \\
\hline$: 35$ & q-perp & $\mathrm{C}$ & 6 & 50.8 \\
\hline 3:05 & q-para & A & 31.2 & 171.9 \\
\hline 35 & q-para & $\mathrm{B}$ & 60.5 & \\
\hline 20 & q-para & $\mathrm{C}$ & 23.4 & \\
\hline 200 & q-para & A & 110.4 & 4.5 \\
\hline 10:05 & q-para & $\mathrm{B}$ & 97.7 & 269.5 \\
\hline$: 05$ & q-perp & $\mathrm{C}$ & 82.0 & 7.8 \\
\hline 35 & q-para & A & 38.1 & .6 \\
\hline 200 & q-para & $\mathrm{B}$ & 46.9 & \\
\hline $1: 05$ & $q-p$ & $\mathrm{C}$ & 25.4 & 78.1 \\
\hline $12: 00$ & q-para & A & 103.5 & 121.1 \\
\hline 2002 & q-para & B & 29.3 & 257.8 \\
\hline 200 & q-perp & $\mathrm{C}$ & 87.9 & 246.1 \\
\hline 200 & q-para & A & & \\
\hline 4:00 & q-para & B & 53.7 & 101.6 \\
\hline 200 & q-perp & $\mathrm{C}$ & 35.2 & 39.1 \\
\hline $10: 35$ & q-para & $\mathrm{B}$ & 54.7 & 335.9 \\
\hline 2002 & q-para & $\mathrm{B}$ & & 7.2 \\
\hline $0: 35$ & q-para & $\mathrm{C}$ & & 156.2 \\
\hline 200 & q-para & $\mathrm{C}$ & 40.0 & 257.8 \\
\hline$-21: 35$ & q-para & $\mathrm{B}$ & 43.0 & 195.3 \\
\hline 0-01:05 & q-perp & $\mathrm{C}$ & 83.0 & 136.7 \\
\hline $0-16: 35$ & q-para & $\mathrm{B}$ & 42.0 & 218.8 \\
\hline 2002-06-02 / 23:20-23:55 & q-perp & $\mathrm{C}$ & 30.3 & 152.3 \\
\hline 2002-06-04 / 06:30-07:05 & q-para & $\mathrm{C}$ & 76.2 & 214.8 \\
\hline 05 / 22:00-22:35 & q-para & $\mathrm{B}$ & 56.6 & 261.7 \\
\hline 2002-06-05 / 11:25-12:00 & q-perp & $\mathrm{C}$ & 54.7 & 74.2 \\
\hline 2002-06-06 / 09:25-10:00 & q-para & $\mathrm{C}$ & 82.0 & 140.6 \\
\hline 2002-06-08 / 10:00-10:35 & q-para & $\mathrm{B}$ & 27.3 & 93.8 \\
\hline 2002-06-08 / 02:00-02:35 & $q-$ & $\mathrm{C}$ & 76.2 & 156.2 \\
\hline
\end{tabular}




\begin{tabular}{lllll} 
2002-06-09 / 00:00-00:35 & q-para & C & 59.6 & 109.4 \\
2002-06-10/07:25-08:00 & q-para & B & 43.0 & 140.6 \\
2002-06-10/03:25-04:00 & q-para & C & 87.9 & 101.6 \\
2002-06-11 / 02:00-02:35 & q-para & C & 72.3 & 168.0 \\
2002-06-13 / 05:25-06:00 & q-para & B & 26.4 & 203.1 \\
2002-06-12 / 19:00-19:35 & q-para & C & 33.2 & 261.7 \\
2002-06-13/16:00-16:35 & q-para & C & 43.0 & 179.7 \\
2002-06-15 / 15:25-16:00 & q-para & B & 53.7 & 250.0 \\
2002-06-15 / 00:00-00:35 & q-para & C & 53.7 & 113.3 \\
2002-06-15 / 23:20-23:55 & q-para & C & 111.3 & 132.8 \\
2002-06-17 / 18:00-18:35 & q-perp & B & 43.9 & 144.5 \\
2002-06-17 / 10:00-10:35 & q-perp & C & 54.7 & 168.0 \\
\hline
\end{tabular}


Table 8.2: Time intervals and dominant frequencies taken from 4-s and 1-s magnetic field data used in the statistical study of the quasi-perpendicular shock upstream waves .

\begin{tabular}{ccc}
\hline Interval & $\begin{array}{c}f_{s c(l o w)} \\
\mathrm{mHz}\end{array}$ & $\begin{array}{c}f_{s c(\text { high })} \\
\mathrm{mHz}\end{array}$ \\
\hline 2002-02-03 / 00:00-00:35 & 99.6 & 453.1 \\
2002-02-03 / 02:30-03:05 & 56.6 & 253.9 \\
2002-02-04 / 09:00-09:35 & 36.1 & 97.7 \\
2002-02-11 / 16:00-16:35 & 47.9 & 222.7 \\
2002-02-11 / 18:00-18:35 & 14.6 & 320.3 \\
2002-02-11 / 20:00-20.35 & 86.9 & 265.6 \\
2002-02-12 / 04:00-04:35 & 60.5 & 296.9 \\
2002-02-12 / 06:00-06:35 & 40.0 & 320.3 \\
2002-02-12 / 08:00-08:35 & 42.0 & 312.5 \\
2002-02-13 / 22:00-22:35 & 49.8 & 97.7 \\
2002-02-16 / 09:00-09:35 & 37.1 & 253.9 \\
2002-02-21 / 00:00-00:35 & 42.0 & 97.7 \\
2002-02-21 / 07:00-07:35 & 33.2 & 273.4 \\
2002-02-21 / 08:00-08:35 & 60.5 & 171.9 \\
2002-02-21 / 20:00-20:35 & 56.6 & 265.6 \\
2002-03-07 / 06:00-06:35 & 55.7 & 269.5 \\
2002-04-23 / 15:30-16:05 & 52.7 & 308.6 \\
2002-04-23 / 17:30-18:05 & 88.9 & 343.8 \\
2002-04-24 / 17:00-17:35 & 43.9 & 97.7 \\
\hline
\end{tabular}





\section{Magnetic turbulence}

\subsection{Introduction}

As the dispersion analyses have shown in Chapter 4, 5, and 7, waves are superposed over different frequencies and wave numbers. The existence of waves with various frequencies and wave numbers prefers the concept of turbulence. Turbulence is ubiquitous. It can be seen by gently stirring cream into coffee, or by observing wave caps at the beach, and it causes the drag on cars and aeroplanes. It is believed that the phenomenon is also widespread in magnetized plasmas.

As presented in Chapter 2, turbulence is often described as a process in which large scale eddies cascade down to smaller scale eddies until a scale is reached on which dissipation sets in. In magnetized plasmas it is not clear how the cascade progresses to the dissipation range, since there are a variety of possible small scale wave modes. Understanding these processes would enable us to determine how energy flows in a turbulent plasma from large scales to the smaller, kinetic scale and thus heats the ambient plasma.

Turbulence in space plasma has been most extensively studied in the solar wind, but the power spectra of the magnetic or velocity field fluctuations could be investigated only in the frequency domain, since the spacecraft have made one- or at best two-point measurements (Scarf et al. 1981, Mellott 1986, Moustaizis et al. 1986, Clasßen et al. 1999, Bavassano Cattaneo et al. 2000, Borovsky et al. 2003). Earlier observations have already shown that power spectra of the magnetic or velocity fields often contained an inertial range with a slope of approximately $-5 / 3$ (Coleman 1966, 1967, 1968), and also different slopes depending on frequency scale: $-1 / 3,-1$, and $-5 / 3$ (Goldstein and Roberts 1999). These observations could not distinguish clearly between a $-5 / 3$ and a $-3 / 2$ slope, as they are close to each other. Here the $-5 / 3$ slope reflects the inertial range spectrum predicted by Kolmogorov (1941) for ideal, isotropic incompressible hydrodynamic turbulence, and the $-3 / 2$ slope reflects the one predicted by Kraichnan (1965) for ideal,incompressible magnetohydrodynamic turbulence. However, various case studies seem to indicate that the spectral slope is more often $-5 / 3$ (Marsch and Tu 1990, Leamon et al. 1998) in the frequency domain, although the solar wind fluctuations display anisotropic and compressible features.

It is not too surprising, as on the distance of $1 \mathrm{AU}$ there is sufficient time for the turbulence to evolve into stationarity. This is not anymore the case when the solar wind develops into shock waves which happens near planetary bow shocks, coronal mass ejec- 
tions, and corotating interaction regions. The best accessible of all those shocks is the Earth's bow shock which evolves in the super-Alfvénic (Mach number $M_{A} \sim 8-10$ ) solar wind stream when it encounters the dipolar geomagnetic field. The presence of the supercritical collisionless bow shock distorts the upstream plasma by reflecting a substantial part of the solar wind plasma back into the solar wind along the interplanetary magnetic field. Interaction between the two oppositely directed plasma streams causes various instabilities leading to the formation of the foreshock in front of the quasi-parallel bow shock, a highly non-quiescent region of wave excitation, damping, and wave particle interactions as shown in Chapter 4, 5, and 6.

This raises the question whether or not the foreshock reaches a quasi-stationary turbulent state, which can best be answered by determining the turbulent wave number spectrum $P(\boldsymbol{k})$. This chapter attempts to determine the turbulence spectra in the wave number domain for the magnetic field fluctuation in the foreshock. The interval is $0805-0840$ UT on February 18, 2002, when inter-spacecraft separation was approximately $100 \mathrm{~km}$. The time interval analyzed in this chapter is the same as that marked as "FS" in Fig. 6.1.

\subsection{Dispersion curves and $k$-spectra}

Determination of wave vectors consists in a search for the highest reliable wave power peaks in $k$-space for a given frequency interval. As the wave telescope provides a wave energy distribution in the $\boldsymbol{k}$-space, it is possible to identify multiple wave vectors contributing and their associated powers. The magnetic fluctuations measured by Cluster are in the frequency range $0.02<\omega_{s c} / 2 \pi<2 \mathrm{~Hz}$, when Cluster was in the foreshock of the quasi-parallel bow shock ( $\omega_{s c}$ is the frequency in the spacecraft frame). The accessible wave number range is $10^{-4} \leq|\boldsymbol{k}|<10^{-1} \mathrm{~km}^{-1}$. We identify the basic wave modes from the experimentally determined dispersion relation $\omega(\boldsymbol{k})=\omega_{s c}-\boldsymbol{k} \cdot \boldsymbol{v}_{\mathrm{f}}$, with $\boldsymbol{v}_{\mathrm{f}}$ the bulk flow velocity which in the present case was $330 \pm 30 \mathrm{~km} \mathrm{~s}^{-1}$.

The results are shown in Figs. 9.1, 9.3, and 9.4. The plasma frame dispersion relation of foreshock low-frequency waves as function of $\omega(|\boldsymbol{k}|)$ is plotted in the upper panel of Fig. 9.1. Indicated are the proton cyclotron frequency $\Omega_{p}=1.1 \mathrm{rad} \mathrm{s}^{-1}$, the ioninertial wave number $k_{i n}=\omega_{p} / c \approx 1.3 \times 10^{-2} \mathrm{~km}^{-1}$, and the thermal ion gyroradius wave number $k_{g y}=\Omega_{p} / v_{t h} \approx 7.3 \times 10^{-3} \mathrm{~km}^{-1}$. Here $\omega_{p}$ is the ion plasma frequency $\Omega_{p}$ the ion cyclotron frequency, $c$ the speed of light, and $v_{t h}$ the ion thermal velocity. Each measurement point is given including error bars. We use the following grid size for scanning in $\boldsymbol{k}$-space: $|\Delta \boldsymbol{k}|=1.3 \times 10^{-4} \mathrm{~km}^{-1}$ for $|\boldsymbol{k}| \leq 1.0 \times 10^{-2} \mathrm{~km}^{-1}$, and $|\Delta \boldsymbol{k}|=4.0 \times 10^{-4} \mathrm{~km}^{-1}$ for $|\boldsymbol{k}|>1.0 \times 10^{-2} \mathrm{~km}^{-1}$. The relative errors in wave number are about $1.3 \%$. The accuracy of the frequency in the plasma frame is determined by the Doppler shift, i.e. $|\Delta \omega / \omega| \leq|\Delta k / k|+\left|\Delta v_{f} / v_{f}\right|$. Relative errors of the velocity are $\sim 14 \%$, yielding $|\Delta \omega / \omega| \sim 15 \%$. For the wave power we estimate the confidence level (Jenkins and Watts 1968). The spatial sample power spectral estimator is essentially a squared spectral estimate with a $\chi^{2}$ distribution, assuming that the sample amplitude 


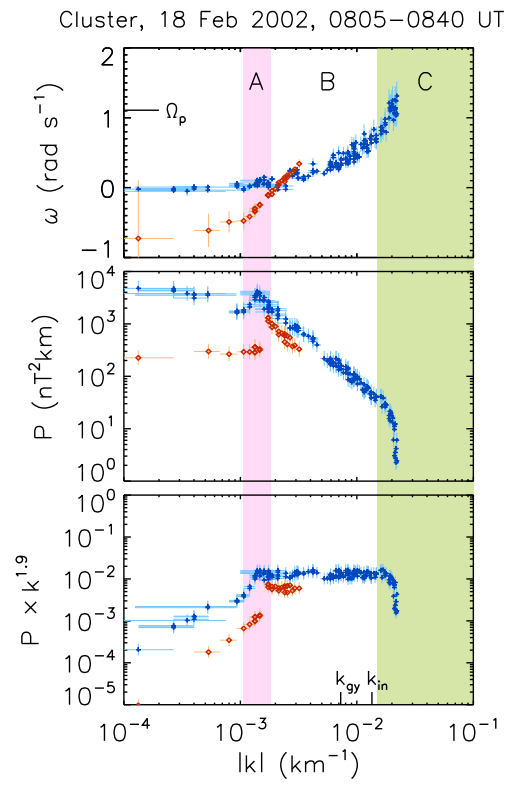

Figure 9.1: Wave frequency in the plasma frame showing (top) and wave power (bottom) as a function of wave number with error estimate. The symbol $\Omega_{p}$ denotes the proton cyclotron frequency. The wave power confidence on the $95 \%$ is also given (vertical error bars in the bottom panel) Region A, B, and C represent the energy injection, the inertial, and the dissipation range, respectively. The symbols $k_{i n}$ and $k_{g y}$ denote the ion inertial wave number and the thermal ion gyroradius wave number, respectively.

distribution is normal. Sixty-four degrees of freedom have been used.

The dispersion relation in Fig. 9.1 exhibits at least two branches shown in red and blue. For small $|\boldsymbol{k}|$ the blue branch starts at frequency close to zero bending upward in frequency with increasing $|\boldsymbol{k}|$. The red branch starts at a negative frequency of $\sim$ $-0.7 \mathrm{rad} \mathrm{s}^{-1}$ before turning up logarithmically $(\omega \sim \ln k)$ and intersecting the blue branch close to wave number $|\boldsymbol{k}| \sim 2 \times 10^{-3} \mathrm{~km}^{-1}$. Negative frequencies imply that the long wavelength waves propagate in the downstream direction. Closer inspection suggests that near intersection of the two branches the blue branch splits and connects to the red branch, indicating wave coupling in region A where the wave power (second panel) maximizes, probably leading to instability which injects energy into turbulence. From wave measurements alone it is not possible to decide what the energy source is as both branches gain in 
power. The most probable source is provided by the shock-reflected ion beam present upstream of the bow shock. Low frequency right-hand polarized whistler waves upstream of the collisionless shock are excited by a right-hand ion-ion beam instability (Gary 1991) and propagate along the background magnetic field. In addition a beam resonant wave propagates along with the ion beam upstream into the solar wind. Dispersion analyses have confirmed that the blue and red branches can approximately be identified with these two wave modes, respectively (Chapter 4).

Toward larger wave numbers away from the energy injection region A the wave power of the whistler branch (second panel) decreases in region B until the cut-off of the spectrum in region $\mathrm{C}$. This decay of the spectrum is described by a power law and thus indicates the existence of a turbulent quasi-inertial range in $P(k) \propto k^{\alpha}$, with $\alpha=-1.9$, of roughly one order of magnitude extension in $|\boldsymbol{k}|$. The third panel shows the formation of a plateau on the spectrum when the spectral decay is compensated by the above power law factor. The cut-off of the spectrum observed in region $\mathrm{C}$ is due to entering the dissipation range. The boundary of this range is close to the gyro- and inertial wave numbers suggesting that gyro-viscosity/cyclotron damping limits the further extension of the inertial range. The beam branch cannot be followed to larger wave numbers. Forming the upper frequency branch of the dispersion relation at wave numbers above injection it ceases to exist even though it also exhibits a narrow quasi-inertial range of slope $\alpha=-2.0$, which is slightly steeper than that of the dominant whistler branch, where most of the available wave energy accumulates. The fact that the spectral slopes are close to -2 indicates intermittency which implies a breakdown of scaling law or self-similarity in the inertial range characterized by a fluctuating energy transfer ratio.

Fig. 9.2 compares our directly determined $|\boldsymbol{k}|$-spectrum with that obtained under Taylor's hypothesis assuming that all the spatial structures are convected by the background flow, and the temporal fluctuations reflect simply the spatial fluctuations. This assumption, of course, cannot be justified under the existence of waves that have certain propagation speeds. Even though both spectra are similar they exhibit distinct differences in shape and structure. The main spectral peaks are disparate by a factor of $\sim 2$ in $|\boldsymbol{k}|$, and the direct spectrum is considerably steeper than the Taylor spectrum. Agreement is reached only at very small $|\boldsymbol{k}|$ for the largest turbulent eddies. Finally, the extension of the Taylor spectrum into very large $|\boldsymbol{k}|>\left(k_{g y}, k_{i n}\right)$ is spurious as at such short wavelengths magnetic turbulence is well in the dissipation range where Taylor's hypothesis fails. We thus conclude that in the foreshock at all wavelengths of interest with the exception of very large eddies the turbulent state is not adequately described by the temporal spectra under Taylor's hypothesis.

We estimate the ratio $r=R_{m, i n} / R_{m, d}$ of magnetic injection and dissipation region Reynolds numbers for the whistler branch assuming that the magnetic viscosity is constant. The wave energy ratio is about $10^{2}$, and the injection to dissipation scale ratio is about 12, yielding $r \approx 8.7 \times 10^{4}$, using Eqs. (4.45) and (5.25) in Biskamp (2003). The Reynolds numbers at injection scales are thus very high. 


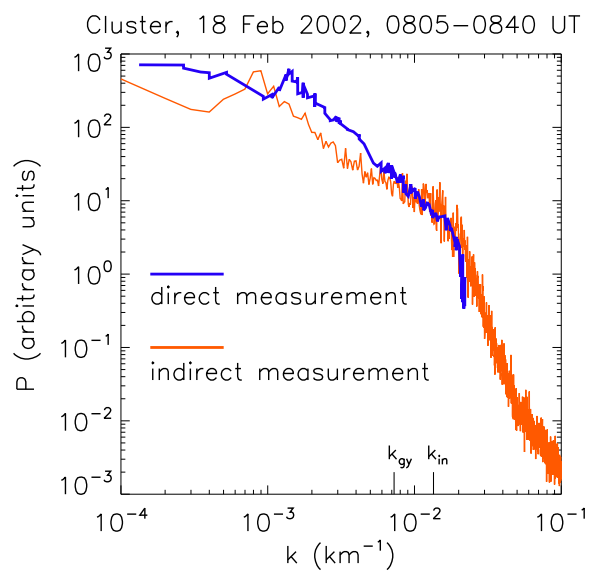

Figure 9.2: Comparison of $k$-spectra between the direct measurement using the wave telescope and the indirect measurement using Taylor's hypothesis.

\section{Anisotropy}

It is of interest to investigate anisotropy of the turbulence distinguishing between compressive, $P_{\|}\left(k_{\perp}\right) \propto\left\langle\left|B_{\|}\right|^{2}\right\rangle$, and non-compressive $P_{\perp}\left(k_{\|}\right) \propto\left\langle\left|B_{\perp}\right|^{2}\right\rangle$ contributions to the power $P(\boldsymbol{k})$. From $\nabla \cdot \boldsymbol{B}=0$ these are given by

$$
P_{\|, \perp}=\frac{P}{1+\tan ^{ \pm 2} \theta},
$$

where $\theta$ is the angle between $k$ and the ambient magnetic field. Figs. 9.3 and 9.4 show the respective dispersion relations and spectra for the non-compressive and compressive modes. Note that the uncertainty increases in both cases since the number of data points is reduced.

The dispersion relation of the non-compressive mode (Fig. 9.3) exhibits the coupling between the two parallel propagating modes at the gap on the dispersion relation around $k_{\|} \approx 2 \times 10^{-3} \mathrm{~km}^{-1}$ which splits the original wave branches. The two small- $k_{\|}$and the two large- $k_{\|}$beam and whistler branches seem to merge to form new dispersion branches, and the intensity on the beam mode maximizes. However, energy injection and inertial ranges are less well indicated in the wave power spectrum. The lower wave number whistler branch still exhibits a narrow quasi-inertial range below $k_{\|} \sim 3 \times 10^{-3} \mathrm{~km}^{-1}$. The higher wave number branch is, however, disparate and a quasi-inertial range cannot be identified. The spectrum exhibits a drop in power almost precisely at $0.5 k_{g y}$ and possibly another one at $2 k_{g y}$. The former may be related to gyro-resonant absorption of wave energy by the plasma at the corresponding wave numbers. The absorbing 


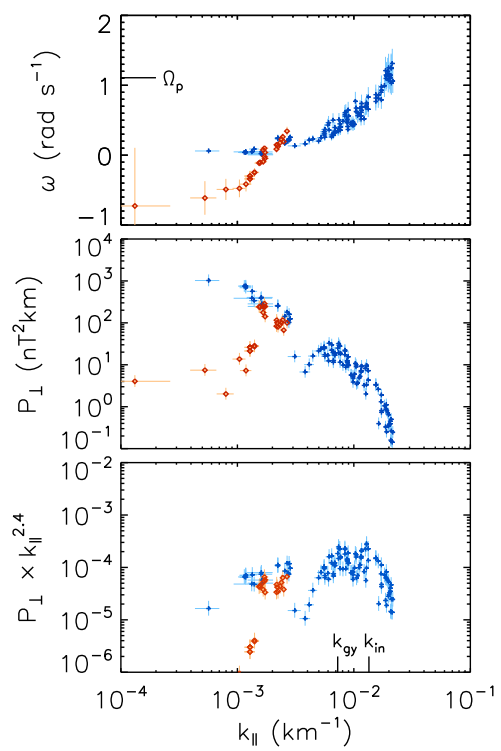

Figure 9.3: The same as Fig. 9.1 for the non-compressive components in dependence of $k_{\|}$.

particles should have roughly four times thermal energy. Because of these absorption lines and the increased scatter determination of a quasi-inertial range is difficult. The spectrum seems to exhibit a break near absorption wave numbers. Larger scales are still consistent with an $\alpha \approx-1.9$ slope, while shorter scales have slope $-5 / 2<\alpha<-9 / 4$ (Fig. 9.3). It is interesting that this range falls into the one theoretically predicted for non-compressive anisotropic magnetohydrodynamic (MHD) turbulence (Biskamp 2003) where $P_{\perp} \propto k^{-5 / 2}$. The smaller value of $\alpha$ obtained here confirms with the expected reduced anisotropy in the kinetic turbulence regime underlying our observations.

The compressive mode dispersion relation (Fig. 9.4) shows that wave coupling in this case occurs at wave numbers $k_{\perp} \approx 10^{-3} \mathrm{~km}^{-1}$ corresponding to longer wavelengths. Due to the scatter of the measurements the splitting of the dispersion relation is less well expressed. Nevertheless, the quasi-inertial range can still be identified in the compressive spectrum from the two lower panels. In particular, the compressive spectrum is more intense than the non-compressive one. They are the compressive modes which bear the main responsibility for the generation of the flat $\alpha \approx-2$ slope on the three-dimensional turbulence in Fig. 9.1. In MHD turbulence (Biskamp 2003) one expects a slope close to $\alpha=-5 / 3$ for compressive turbulence. This is not realized here except possibly for the 


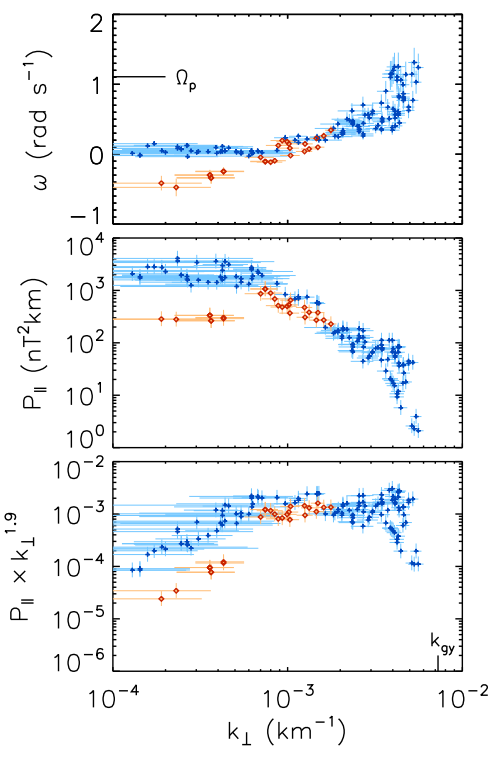

Figure 9.4: The same as Fig. 9.1 for the compressive components in dependence of $k_{\perp}$. Note that the non-compressive intensity is one order of magnitude less than the compressive one.

short range of the red part of the spectrum, if taken separately.

\subsection{Discussion}

Turbulence in the foreshock consists of the general solar wind turbulence and superimposed resonant wave modes excited in the foreshock boundary by the interaction between the solar wind and intense streams of bow shock reflected particles. The wave modes reach large amplitudes depending on how long they stay in resonance along the foreshock boundary. Entering the foreshock by convection with the solar wind toward the bow shock, they run out of resonance, start interacting with other waves, steepen to form nonlinear structures the long-wavelength part of which participates in shock reformation, while the shorter wavelengths enter the turbulent cascade. In the latter case the relation between frequency $\omega$ and wavenumber $\boldsymbol{k}$ looses its meaning of a linear dispersion relation determining one single wave mode. Whether or not an inertial range develops depends on the time the wave energy has to cascade down from injection to dissipation scales. In the bounded foreshock region this time is relatively short not allowing for the develop- 


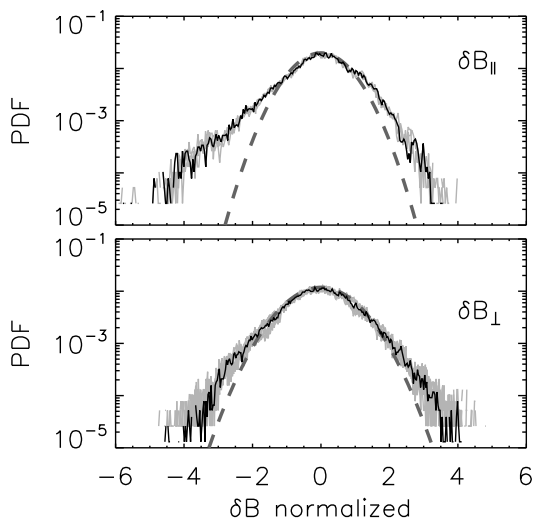

Figure 9.5: Probability distribution function (PDF) of the magnetic fields for the compressive part $\left(\delta B_{\|}\right)$and the non-compressive part $\left(\delta B_{\perp}\right)$, plotted on the normalized (to the Gaussian half-width) fluctuation amplitude. Solid curves in gray and in black represent PDF superposed for four s/c data and PDF averaged over four s/c data, respectively. Dashed curves are Gaussian-fittings around $\delta B=0$.

ment of stationary turbulence. One expects the turbulence to be in an intermittent state, not fully developed state exhibiting a true inertial range. The actual spectral slope should be steeper than Kolmogorov's inertial range slope with $\alpha=\frac{5}{3}$ or Kraichnan's slope with $\alpha=\frac{3}{2}$. This is reflected in the observed spectral indices $\alpha \approx-1.9$ for the dominant compressive and $\alpha>-\frac{5}{2}$ for the non-compressive turbulent spectra which indicate that the turbulence is intermittent possessing a still developing forward cascading quasi-inertial short wavelength range. Thus, in addition to (nonlinear) wave modes, the foreshock contains a substantial level of turbulence which is in an evolutionary intermittent state. Intermittency is also indicated by the bump on the end of this quasi-inertial range visible on the compressive spectrum. Turbulent energy is accumulating at the scale $k_{\perp}<k_{g y}$ just before entering the dissipative range. If the foreshock would be spatially more extended, flattening of the spectrum should proceed from here backward until equilibrium would be reached, possibly in the Kolmogorov states. In addition, further evidence for foreshock turbulence being intermittent is provided by the shapes of the probability distribution functions (PDFs) of the magnetic fluctuations shown in Fig. 3. These PDFs exhibit well expressed non-Gaussian wings at large-fluctuation amplitudes and asymmetries in both the compressive and non-compressive components for all four spacecraft typical for intermittency. 


\subsection{Summary}

The direct experimental determination of $k$-spectra of magnetic turbulence in the high- $\beta$ plasma upstream of a collisionless shock has been presented. The high-time resolution measurements have been taken with Cluster at spacecraft separation of $\sim 100 \mathrm{~km}$. The spectra exhibit three dynamnic ranges: an energy injection range at $k \sim 10^{-3} \mathrm{~km}^{-1}$, a quasi-inertial range $10^{-3}<k<10^{-2} \mathrm{~km}^{-1}$, and an indication of the dissipation range $k>10^{-2} \mathrm{~km}^{-1}$. Dissipation starts close to the gyro-wave number suggesting that ionviscosity is the main dissipative process. Compressive turbulence dominates the spectrum with slope $\alpha \approx-1.9$ suggesting intermittent non-stationary turbulence. 



\section{Summary and outlook}

Measurements and observations made by the four Cluster spacecraft have been analyzed in pursuing low frequency wave characteristics upstream and downstream of the terrestrial bow shock. Shock waves are known to be found in many places in the Universe and to play an important role in accelerating cosmic ray particles. Studying the Earth's bow shock is of significant importance, for it allows us to achieve detailed in-situ observations whose results have immediate astrophysical implications. The concept of the collisionless shocks have been presented in Chapter 2 together with reviews of the upstream and downstream waves, and turbulence. An overview of the Cluster spacecraft mission and reviews of the principles of magnetic field measurements and particle measurements has been presented in Chapter 3. The methods of wave analyses using single and multi-point measurements have been given in Chapter 3, too. The wave telescope technique has been extensively used and allowed not only to calculate wave powers associated with frequencies and wave numbers but also to determine phase velocities, rest frame frequencies, dispersion curves, propagation patterns, and turbulence spectra.

Chapter 4 has presented the dispersion analysis of the upstream waves. The wave dispersion relation was determined experimentally and showed a good agreement with the one calculated for the ion beam plasma model, suggesting that the upstream waves represent a whistler and a beam resonant wave. This is one of the examples of wave mode identification and confirms the physical processes drawn by the earlier studies that some upstream ions are specularly reflected at the shock and flow against the incoming ions, while they form an unstable particle distribution in velocity space which drives waves and collapses into a stable state.

Chapter 5 has presented an example of wave-particle interaction. The dispersion and polarization analysis indicated the existence of the whistler wave in the upstream region. The wave was accompanied by nongyrotropic electrons which were trapped by the wave field. While it is known that some beam ions are phase bunched by the wave field, there is also an interplay between the waves and the electrons.

Chapter 6 has presented the statistical study of the upstream waves. It was shown that the wave phase velocities in the plasma rest frame were oriented toward upstream along the magnetic field. This direction is the same as that of the backstreaming ions.

Chapter 7 has presented the dispersion analysis of the downstream waves. The dispersion curves were investigated along a Cluster orbit and showed a transition from the whistler and the beam resonant wave in the upstream region to the mirror mode in the 
downstream region. The upstream waves are not transmitted across the shock, as they are swept by the solar wind toward downstream.

Chapter 8 has presented the statistical study of the downstream waves. While the upstream waves propagated parallel to the background magnetic field, the downstream waves propagated perpendicular. The mirror mode properties were frequently detected in the downstream region but they had finite propagation speeds, possibly coupled to the background inhomogeneities. In the statistical average there is an organization in wave propagation pattern: outward divergent in the upstream region; toward the magnetosheath flank region aligned with the plasma flow direction in the downstream region; and inward convergent in the magnetosheath flank.

Chapter 9 has attempted to determine the spectra of magnetic turbulence directly in the wave number domain, showing three ranges appearing in the classical turbulence picture: energy injection, inertial, and dissipation range. The inertial range spectrum was not the classical Kolmogorov type or the Kraichnan type with the slope $-5 / 3$ and $-3 / 2$, respectively, but it showed an almost -2 slope, indicating that the fluctuations are intermittent and not fully developed turbulence. It was also understood on what scales the waves damp and thermalize the ambient plasma. Magnetic viscosity dominates on scale close to the ion inertial length or the thermal ion gyroradius.

The disturbance in the plasma caused by the bow shock leads to wave excitation both in the upstream and the downstream regions. The studies given in this thesis suggest that ultimately ion reflection is the cause of wave excitation in both regions, but as the wave properties are different between them, the physical processes are accordingly different. The upstream waves propagate parallel to the magnetic field and are identified as the one driven by the ion beam instability, while the downstream waves propagate perpendicular and represent the mirror modes, possibly coupled to the background inhomogeneities. On the other hand they exhibit a unique propagation pattern imposed by the background magnetic field topology in those regions. Investigating spatial scales is essential in space plasma research. Dispersion relations and propagation patterns shown in the thesis may be associated with planetary bow shocks, interplanetary shocks, and also shocks on astrophysical scales.

The thesis has attempted to answer a number of questions on waves and turbulence in space, but it has raised on the other hand subjects that are left as open questions. They are summarized as an outlook below.

(1) Are the upstream waves mode-converted at the shock? It was understood in Chapter 7 that the upstream waves (foreshock waves) were not transmitted into the downstream region. Are the waves mode-converted into different kinds of waves across the shock, or are they simply lost? In the latter case, into what energy the upstream wave energy is changed?

(2) Why were the ion cyclotron waves not clearly detected? Neither the dispersion analysis nor the statistical study showed a clear indication of the ion cyclotron waves. The ion cyclotron waves have a larger growth rate for temperature anisotropy instabilities, but observationally the mirror modes dominate in the magnetosheath. Is it simply because 
the former prefers a low value of plasma parameter $\beta$, while the latter prefers a high $\beta$ ? Since developed mirror modes exhibit magnetic bottle structures where $\beta$ may be locally low, it could be possible to detect the ion cyclotron waves in the magnetosheath.

(3) Is there any dependence on the Mach number or $\beta$ about the upstream and downstream waves? It is known that the dynamics and the structure of a collisionless shock depend not only on the shock angles (between the upstream magnetic field and the shock normal) but also the Mach numbers and $\beta$. In the statistical study wave properties were investigated with respect to the shock angle for the quasi-parallel and the quasi-perpendicular shock regimes, but no clear difference was found. However, we do not know yet if wave excitation, modes, and propagation speeds and directions depend on these two parameters.

(4) Technically, the spacecraft separation determines the largest wave number available in the wave telescope analysis. In the case of Cluster one has at best $100 \mathrm{~km}$ separation, which is roughly a typical scale of ion dynamics (thermal gyroradius or inertial length) in the solar wind and in the magnetosheath. This means that the electron scale (less than $10 \mathrm{~km}$ ) can not be resolved by Cluster, although its importance can be widely acknowledged (e.g. Chapter 5). Future multi-spacecraft missions like MMS (Magnetospheric Multi-Scale mission) will enable us to access the electron scale physics.

(5) It was presented that the turbulence spectra can be determined directly in the wave number domain. The wave number spectra shall be investigated in various regimes of space plasma: in the undisturbed solar wind and in the magnetosheath, where it is thought that the Alfvén and the mirror mode turbulence may exist.

(6) The mode recognition method (Glassmeier et al. 1995), that is to identify energy contributions from the fast mode, intermediate mode, and slow mode waves, shall be applied to the waves in the shock upstream and downstream regions.

(7) Of course, all the methods presented in the thesis can be applied to other regions of the magnetosphere. For instance, waves in the magnetotail or in the current sheets would be an interesting subject. Dispersion curves and propagation patterns of the substormrelated waves shall be determined in future. The THEMIS mission (Time History of Events and Macroscale Interactions during Substorms) will answer fundamental questions regarding the magnetospheric substorm instability.

In analyzing waves from in-situ observations in space, I conclude the $\mathrm{PhD}$ thesis by saying that multi-point measurements are not only a powerful means but also an essential means. Future multi-spacecraft missions are promising to better understand physical processes of waves, instabilities, and turbulence in the collisionless plasma. 



\section{Bibliography}

Anderson, B. J., S. A. Fuselier, S. A., and D. Murr, Electromagnetic ion cyclotron waves observed in the plasma depletion layer, Geophys. Res. Lett., 18, 1955-1958, 1991.

Anderson, B. J., S. A. Fuselier, S. P. Gary, and R. E. Denton, Magnetic spectral signatures in the Earth's magnetosheath and plasma depletion layer, J. Geophys. Res., 99, 5877$5891,1994$.

Arthur, C. W., R. L. McPherron, and J. D. Means, A comparative study of three techniques for using the spectral matrix in wave analysis, Radio Sci., 11, 833-845, 1976.

Asbridge, J. R, S. J. Bame, and I. B. Stron, Outward flow of protons from the Earth's bow shock, J. Geophys. Res., 73, 5777-5782, 1968.

Balikhin, M. A., T. D. de Wit, H. S. C. K. Alleyne, L. J. C. Woolliscroft, S. N. Walker, V. Krasnosel'skikh, W. A. C. Mier-Jedrzejeowicz, and W. Baumjohann, Experimental determination of the dispersion of waves observed upstream of a quasi-perpendicular shock, Geophys. Res. Lett., 24, 787-790, 1997a.

Balikhin, M. A., L. J. C. Woolliscroft, H. St. C. Alleyne, M. Dunlop, and M. A. Gedalin, Determination of the dispersion of low frequency waves downstream of a quasiperpendicular collisionless shock, Ann. Geophysicae, 15, 143-151, $1997 \mathrm{~b}$.

Balikhin, M. A., S. Schwartz, S. N. Walker, H. St. C. K. Alleyne, M. Dunlop, and H. Lühr, Dual-spacecraft observations of standing waves in the magnetosheath, J. Geophys. Res., 106, 25,395-408, 2001.

Balogh, A., M. W. Dunlop, S. W. H. Cowley, D. J. Southwood, et al., The Cluster magnetic field investigation, Space Sci. Rev., 79, 65-91, 1997.

Balogh, A., Carr, C.M., Acuña, M.H., Dunlop, M.W., T. J. Beek, P. Brown, K.-H. Fornaçon, E. Georgescu, K.-H. Glassmeier, J. Harris, G. Musmann, T. Oddy, K. Schwingenschuh, The Cluster magnetic field investigation: overview of in-flight performance and initial results, Ann. Geophysicae, 19, 1207-1217, 2001.

Barnes, A., Theory of generation of bow-shock-associated hydromagnetic waves in the upstream interplanetary medium, Cosmic Electrodyn., 1, 90-114, 1970. 
Baumjohann, W., and R. A. Treumann, Basic space plasma physics, Imperial College Press, London, 1997.

Bavassano Cattaneo, M. B., G. Moreno, G. Russo, and J. D. Richardson, MHD turbulence in Saturn's magnetosheath downstream of a quasi-parallel bow shock, J. Geophys. Res., $105,23,141-152,2000$.

Bendat, J. S., and A. G. Piersol, Engineering applications of correlation and spectral analysis, pp. 54-56, John Wiley \& Sons, Inc., New York, 1980.

Biskamp, D., and H. Welter, Ion heating in high-Mach-number, oblique, collisionless shock waves, Phys. Rev. Lett., 28, 410-413, 1972.

Biskamp, D., Magnetohydrodynamic Turbulence, Cambridge University Press, Cambridge, 2003.

Born, M. and E. Wolf, Principles of optics, 6th ed., pp. 503-504, 550, Pergamon press, New York, 1980.

Borovsky, J. E., and H. O. Funsten, MHD turbulence in the Earth's plasma sheet: dynamics, dissipation, and driving, J. Geophys. Res., 108, 1284, doi:10.1029/2002JA09625, 2003.

Burgess, D., Collisionless shocks, Introduction to space physics, (Eds) M. G. Kivelson and C. T. Russell, pp. 129-163, Cambridge University Press, United Kingdom, 1995.

Burgess, D., What do we really know about upstream waves?, Adv. Space Res., 20, 673682, 1997.

Cairns, I. H., D. H. Fairfield, R. R. Anderson, V. E. H. Carlton, K. I. Paularena, and A. J. Lazarus, Unusual location of Earth's bow shock on September 24-25, 1987: Mach number effects, J. Geophys. Res., 100, 47-62, 1995.

Claßen, H.-T, G. Mann, R. J. Forsyth, and E. Keppler, Low frequency plasma turbulence and high energy particles at CIR-related shock waves, Astron. Astrophys., 347, 313320, 1999.

Coleman, P. J., Hydromagnetic waves in the interplanetary plasma, Phys. Rev. Lett., 17, 207-211, 1966.

Coleman, P. J., Wave-like phenomena in the interplanetary plasma: Mariner 2, Planet. Space Sci., 15, 953, 1967.

Coleman, P. J., Turbulence, viscosity, and dissipation in the solar-wind plasma, Astrophys. J., $153,371,1968$. 
Denton, R. E., S. P. Gary, X. Li, B. J. Anderson, J. W. Labelle, and M. Lessard, Lowfrequency fluctuations in the magnetosheath near the magnetopause, J. Geophys. Res., 100, 5665-5679, 1995.

Denton, R. E., M. R. Lessard, J. W. Labelle, and S. P. Gary, Identification of lowfrequency magnetosheath waves, J. Geophys. Res., 103, 23,661-676, 1998.

Dudok de Wit, T., V. V. Krasnosel'skikh, S. D. Bale, M. W. Dunlop, H. Lühr, S. J. Schwartz, and L. J. C. Woolliscroft, Determination of dispersion relations in quasistationary plasma turbulence using dual satellite data, Geophys. Res. Lett., 22, 26532656, 1995.

Dubinin, E., K. Sauer, and J. F. McKenzie, Nonlinear stationary waves and solitons in ion beam-plasma configuration, J. Geophys. Res., 109, A02208, 2004.

Eastwood, J. P., A. Balogh, M. W. Dunlop, T. S. Horbury, and I. Dandouras, Cluster observations of fast magnetosonic waves in the terrestrial foreshock, Geophys. Res. Lett., 29, 2046-2049, 2002.

Eastwood, J. P., A. Balogh, E. A. Lucek, C. Mazelle, and I. Dandouras, On the existence of Alfvén waves in the terrestrial foreshock, Ann. Geophysicae, 21, 1457-1465, 2003.

Escoubet, C.P., M. Fehringer, and M. Goldstein, The Cluster mission, Ann. Geophysicae, 19, 1197-1200, 2001.

Fairfield, D., Bow shock associated waves observed in the far upstream interplanetary medium, J. Geophys. Res., 74, 3541-553, 1969.

Fairfield, D. H., Whistler waves observed upstream from collisionless shocks, J. Geophys. Res., 79, 1368-1378, 1974.

Farris, M. H., and C. T. Russell, Determining the standoff distance of the bow shock: Mach number dependence and use of models, J. Geophys. Res., 99, 17,681-689, 1994.

Farris, M. H., C. T. Russell, C. T., and M. F. Thomsen, Wave activity associated with the low beta collisionless shock, in Solar wind sources of magnetospheric ultra-lowfrequency waves, pp. 99, Geophysical Monograph 81, (Eds) M. J. Engrebretson, K. Takahashi and M. Scholer, Amer. Geophys. Union, 1994.

Farrugia, C. J., G. Gnavi, F. T. Gratton, H. Matsui, R. B. Torbert, R. P. Lepping, N, Oieroset, and R. P. Lin, Electromagnetic ion cyclotron waves in the subsolar region under normal dynamic pressure: Wind observations and theory, J. Geophys. Res., 109, A02202, 2004.

Fowler, R.A., B. J. Kotick, and R. D. Elliott, Polarization analysis of natural and artificially induced geomagnetic micropulsations, J. Geophys. Res., 72, 2871-2883, 1967. 
Frisch, U., Turbulence: the legacy of A. N. Kolmogorov, Cambridge University Press, Cambridge, 1995.

Fuselier, S. A., Energetic magnetospheric protons in the plasma depletion layer, J. Geophys. Res., 97, 13,759-13,766, 1992.

Fuselier, S. A., M. F. Thomsen, S. P. Gary, S. J. Bame, C. T. Russell, and G. K. Parks, The phase relationship between gyrophase-bunched ions and MHD-like waves, Geophys. Res. Lett., 13, 60-63, 1986.

Gary, S. P., J. T. Gosling, and D. W. Forslund, The electromagnetic ion beam instability upstream of the Earth's bow shock, J. Geophys. Res., 86, 6691-6696, 1981.

Gary, S. P., Low-frequency waves in a high-beta collisionless plasma: polarization, compressibility and helicity, J. Plasma Phys., 35, 431-447, 1986.

Gary, S. P., Electromagnetic ion/ion instabilities and their consequences in space plasmas - A review, Space Sci. Rev, 56, 373-415, 1991.

Gary, S. P., The mirror and ion cyclotron anisotropy instabilities, J. Geophys. Res., 97, 8519-8529, 1992.

Gary, S., Theory of space plasma microinstabilities. Cambridge; Cambridge Atmos. Space Science Series, 1993.

Gary, S. P., S. A. Fuselier, and B. J. Anderson, Ion anisotropy instabilities in the magnetosheath, J. Geophys. Res., 98, 1481-1488, 1993.

Gary, S. P., and D. Winske, Simulations of ion cyclotron anisotropy instabilities in the terrestrial magnetosheath, J. Geophys. Res., 98, 9171-9179, 1993.

Gary, S. P., P. D. Convery, R. E. Denton, S. A. Fuselier, and B. J. Anderson, Proton and helium cyclotron anisotropy instability thresholds in the magnetosheath, J. Geophys. Res., 99, 5915-5921, 1994a.

Gary, S. P., M. E. McKean, D. Winske, B. J. Anderson, R. E. Denton, and S. A. Fuselier, The proton cyclotron instability and the anisotropy/B inverse correlation, J. Geophys. Res., 99, 5903-5914, 1994b.

Gary, S. P, and M. A. Lee, The ion cyclotron anisotropy instability and the inverse correlation between proton anisotropy and proton beta, J. Geophys. Res., 99, 11,297-11,301, 1994.

Gedalin, M., Yu. E. Lyubarsky, M. Balikhin, and C. T. Russell, Mirror modes: nonMaxwellian distributions, Phys. Plasmas, 8, 2934-2945, 2001. 
Gedalin, M., M. Balikhin, R. J. Strangeway, and C. T. Russell, Long-wavelength mirror modes in multispecies plasmas with arbitrary distributions, J. Geophys. Res., 107, A2, doi:10.1029/2001JA000178, 2002.

Glassmeier, K.-H., and U. Motschmann, Comments on time-series analysis, Proc. of the workshop on data analysis tools, (Eds) Glassmeier, K.H., Motschmann, U., and Schmidt, R., ESA SP-371, 7-14, ESA Publications Division, The Netherlands, 1995.

Glassmeier, K.-H., U. Motschmann, and R. v. Stein, Mode recognition of MHD wave fields at incomplete dispersion measurements, Ann. Geophysicae, 13, 76-83, 1995.

Glassmeier, K.-H., U. Motschmann, M. Dunlop, A. Balogh, M. H. Acuña, C. Carr, G. Musmann, K.-H. Fornaçon, K. Schweda, J. Vogt, E. Georgescu, and S. Buchert, Cluster as a wave telescope - first results from the fluxgate magnetometer, Ann. Geophysicae, 19, 1439-1447, 2001 (Correction, Ann. Geophysicae, 21, 1071, 2003).

Goldstein, M. L., and D. A. Roberts, Magnetohydrodynamic turbulence in the solar wind, Phys. Plasmas, 6, 4154-4160, 1999.

Gosling, J. T., J. R. Asbridge, S. J. Bame, G. Paschmann, and N. Sckopke, Observations of two distinct populations of bow shock ions in the upstream solar wind, Geophys. Res. Lett., 5, 957-960, 1978.

Greenstadt, E. W., I. M. Green, G. T. Inouye, A. J. Hundhausen, S. J. Bame, and I. B. Strong, Correlated magnetic field and plasma observations of the Earth's bow shock, $J$. Geophys. Res., 73, 51-60, 1968.

Gurgiolo, C., M. L. Goldstein, Y. Narita, K.-H. Glassmeier, and A. N. Fazakerley, A phase locking mechanism for nongyrotropic electron distributions upstream of the Earth's bow shock, J. Geophys. Res., 110, A06206, 2005.

Hasegawa, A., Drift mirror instability in the magnetosphere, Phys. Fluids, 12, 2642, 1969.

Hasegawa, A., and L. Chen, Theory of the drift mirror instability, Plasma waves and instabilities at comets and in magnetospheres, pp. 173-177, Washington DC, Amer. Geophys. Union, Geophysical Monograph Series, 1989.

Hassam, A. B., Transmission of Alfvén waves through the Earth's bow shock: theory and observation, J. Geophys. Res., 83, 643-653, 1978.

Hellinger, P., A. Mangeney, and A. Matthews, Whistler waves in 3D hybrid simulations of quasiperpendicular shocks, Geophys. Res. Lett, 23, 621-624, 1996.

Hellinger, P., P. Trávníček, and V. I. Sotnikov, Whistler waves in the foot region of perpendicular shock: hybrid simulations, Proc. of 6th European Workshop on Collisionless Shock, pp. 29, 2005. 
Hill, P., G. Paschmann, R. A. Treumann, W. Baumjohann, N. Sckopke, and H. Lühr, Plasma and magnetic field behavior across the magnetosheath near local noon, J. Geophys. Res., 100, 9575-9584, 1995.

Hoppe, M. M., C. T. Russell, L. A. Frank, T. E. Eastman, and E. W. Greenstadt, Upstream hydromagnetic waves and their association with backstreaming ion populations - ISEE 1 and 2 observations, J. Geophys. Res., 86, 4471-4492, 1981.

Hoppe, M. M., and C. T. Russell, Plasma rest frame frequencies and polarizations of the low-frequency upstream waves - ISEE 1 and 2 observations, J. Geophys. Res., 88, 2021-2027, 1983.

Hubert, D., C. C. Harvey, and C. T. Russell, Observations of magnetohydrodynamic modes in the earth's magnetosheath at 0600 LT, J. Geophys. Res., 94, 17,305-309, 1989.

Hubert, D., C. Lacombe, C. C. Harvey, M. Moncuquet, C. T. Russell, and M. F. Thomsen, Nature, properties, and origin of low-frequency waves from an oblique shock to the inner magnetosheath, J. Geophys. Res., 103, 26,783-26,798, 1998.

Iroshnikov, P. S., Turbulence of a conducting fluid in a strong magnetic field, Sov. Astron., 7, 566-571, 1964.

Jenkins, G. M., and D. G. Watts, Spectral analysis and its applications, pp. 313-317, Holden-Day, San Francisco, 1968.

Johnson, J. R., and C. Z. Cheng, Global structure of mirror modes in the magnetosheath, J. Geophys. Res., 102, 7179-7189, 1997.

Johnstone, A. D., and C. Alsop, and S. Burge, P. J. Carter, A. J. Coates, A. J. Coker, A. N. Fazakerley, N. Grande, R. A. Gowen, C. Gurgiolo, B. K. Hancock, B. Narheim, A. Preece, P. H. Sheather, J. D. Winningham, and R. D. Woodliffe, PEACE: a plasma electron and current experiment, Space Sci. Rev., 79, 351-398, 1997.

Kennel, C. F., J. P. Edmiston, and T. Hada, A quarter century of collisionless shock research, in Collisionless shocks in the heliosphere: a tutorial review, Geophys. Monogr. Ser., 34, (Eds) R. G. Stone and B. T. Tsurutani, pp. 1-36, Amer. Geophys. Union, Washington, DC, 1985.

Kivelson, M. G., Pulsation and magnetohydrodynamic waves, in Introduction to space physics, pp. 330-355, (Eds) M. G. Kivelson and C. T. Russell, Cambridge University Press, 1995.

Kivelson, M. G., and D. J. Southwood, Mirror instability II: the mechanism of nonlinear saturation, J. Geophys. Res., 101, 17,365-17,371, 1996. 
Kolmogorov, A. N., The local structure of turbulence in incompressible viscous fluid for very large Reynolds number, Dokl. Akad. Nauk SSSR, 30, 9-13, 1941 (reprinted in Proc. R. Soc. Lond. A 434, 9-13, 1991).

Kraichnan, R. H., Inertial range spectrum in hydromagnetic turbulence, Phys. Fluids, 8, 1385-1387, 1965.

Krall, N. A., What do we really know about collisionless shocks?, Adv. Space Res., 20, 715-524, 1997.

Krasnosel'skikh, V., Nonlinear motions of a plasma across a magnetic field, Sov. Phys. Jetp., 62, 282, 1985.

Krasnosel'skikh, V. V., B. Lembège, P. Savoini, and V. V. Lobzin, Nonstationarity of strong collisionless quasiperpendicular shocks: theory and full particle numerical simulations, Phys. Plasmas, 9, 1192-1209, 2002.

Krauss-Varban, D., and N. Omidi, Structure of medium Mach number quasi-parallel shocks - upstream and downstream waves, J. Geophys. Res., 96, 17,715-731, 1991.

Krauss-Varban, D., and N. Omidi, Propagation characteristics of waves upstream and downstream of quasi-parallel shocks, Geophys. Res. Lett. 20, 1007-1010, 1993.

Krauss-Varban, D., Bow shock and magnetosheath simulations: wave transport and kinetic properties, Solar wind sources of magnetospheric ultra-low-frequency waves, pp. 121-134, Geophysical Monograph 81, Amer. Geophys. Union, 1994.

Krauss-Varban, D., N. Omidi, and K. B. Quest, Mode properties of low-frequency waves: kinetic theory versus Hall-MHD, J. Geophys. Res., 99, 5987-6009, 1994.

Leamon, R. J., C. W. Smith, N. F. Ness, and W. H. Matthaeus, Observational constraints on the dynamics of the interplanetary magnetic field dissipation range, J. Geophys. Res., 103, 4775-4787, 1998.

Marsch, E., and C.-Y. Tu, On the radial evolution of MHD turbulence in the inner heliosphere, J. Geophys. Res., 95, 8211-8229, 1990.

Marsden, R. G., E. J. Smith, J. F. Cooper, and C. Tranquille, Ulysses at high heliographic latitudes: an introduction, Astron. Astrophys., 316, 279, 1996.

Matsuoka, A., D. J. Southwood, S. Kokubun, and T. Mukai, Propagation sense of lowfrequency MHD waves in the magnetosheath observed by Geotail, J. Geophys Res., 105, 18,361-376, 2000.

Matthaeus, W. H. and M. L. Goldstein, Measurement of the rugged invariants of magnetohydrodynamic turbulence in the solar wind, J. Geophys. Res., 87, 6011-6028, 1982. 
Matsukiyo, S., and M. Scholer, Modified two-stream instability in the foot of high Mach number quasi-perpendicular shocks, J. Geophys. Res., 108, SMP 19-1, 2003.

Mazelle, C., D. Queéau, and K. Meziane, Nonlinear wave particle interactions due to quasi-monochromatic ULF waves in the Earth foreshock, Nonlinear Processes in Geophys., 7, 185-190, 2000.

McKenzie, J. F., and K. O. Westphal, Transmission of Alfvén waves through the Earth's bow shock, Planet. Space Sci., 17, 1029-1037, 1969.

McKenzie, J. F., and K. O. Westphal, Interaction of hydromagnetic waves with hydromagnetic shocks, Phys. Fluids, 13, 630-640, 1970.

McPherron, R. L., Magnetospheric dynamics, Introduction to space physics, pp. 448, (Eds) M. G. Kivelson and C. T. Russell, Cambridge University Press, 1995.

Mellott, M. M., Plasma wave signatures of collisionless shocks and the role of plasma wave turbulence in shock formation, Adv. Space Res., 6, 25-32, 1986.

Merka, J., A. Szabo, A., T. W. Narock, J. H. King, K. I. Paularena, J. D. Richardson, A comparison of IMP 8 observed bow shock positions with model predictions, J. Geophys. Res., 108, SMP6-1, CiteID 1077, doi:10.1029/2002JA009384, 2003.

Meziane, K., C. Mazelle, C. D’Uston, H. Rème, R. P. Lin, C. W. Carlson, D. Larson, J. P. McFadden, R. E. Ergun, K. A. Anderson, G. K. Parks, D. Berdichevsky, and R. P. Lepping, Wind observation of gyrating-like ion distributions and low frequency waves upstream from the earth's bow shock, Adv. Space Res., 20, 703-706, 1997.

Meziane, K., C. Mazelle, R. P. Lin, D. LeQuéau, D. E. Larson, G. K. Parks, and R. P. Lepping, Three-dimensional observations of gyrating ion distributions far upstream from the Earth's bow shock and their association with low-frequency waves, J. Geophys. Res., 106, 5731-5742, 2001.

Möbius, E., H. Kucharek, C. Mouikis, E. Georgescu, L. M. Kistler, M. A. Popecki, M. Scholer, J. M. Bosqued, H. Rème, C. W. Carlson, B. Klecker, A. Korth, G. K. Parks, J. C. Sauvaud, H. Balsiger, M.-B. Bavassano-Cattaneo, I. Dandouras, A. M. Dilellis, L. Eliasson, V. Formisano, T. Horbury, W. Lennartsson, R. Lundin, M. McCarthy, J. P. McFadden, and G. Paschmann, Observations of the spatial and temporal structure of field-aligned beam and gyrating ring distributions at the quasi-perpendicular bow shock with Cluster CIS, Ann. Geophysicae, 19, 1411-1420, 2001.

Motschmann, U., T. I. Woodward, K.H. Glassmeier, and M. W. Dunlop, Array signal processing techniques, Proc. CLUSTER workshop on data analysis tools, pp. 79-86, (Eds) Glassmeier, K.H., Motschmann, U., and Schmidt, R., ESA SP-371, ESA Publications Division, The Netherlands, 1995. 
Motschmann, U., T. I. Woodward, K. H. Glassmeier, D. J. Southwood, and J.L. Pinçon, Wavelength and direction filtering by magnetic measurements at satellite arrays: generalized minimum variance analysis, J. Geophys. Res., 101, 4961-4965, 1996.

Moustaizis, S., D. Hubert, A. Mangeney, C. C. Harvey, C. Perche, and C. T. Russell, Magnetohydrodynamic turbulence in the Earth magnetosheath, Ann. Geophysicae, 4, 355-362, 1986.

Narita, Y., K.-H. Glassmeier, S. Schäfer, U. Motschmann, K. Sauer, I. Dandouras, K.H. Fornaçon, E. Georgescu, and H. Rème, Dispersion analysis of ULF waves in the foreshock using cluster data and the wave telescope technique, Geophys. Res. Lett., 30, SSC 43-1, 2003.

Narita, Y., K.-H. Glassmeier, S. Schäfer, U. Motschmann, M. Fränz, I. Dandouras, K.H. Fornaçon, E. Georgescu, A. Korth, H. Rème, and I. Richter, Alfvén waves in the foreshock propagating upstream in the plasma rest frame: statistics from Cluster observations, Ann. Geophysicae 22, 2315-2323, 2004.

Narita, Y., and K.-H. Glassmeier, Dispersion analysis of low-frequency waves through the terrestrial bow shock, J. Geophys. Res., 110, A12215, doi:10.1029/2005JA011256, 2005 .

Narita, Y. , K.-H. Glassmeier, K.-H. Fornaçon, I. Richter, S. Schäfer, U. Motschmann, I. Dandouras, H. Rème, and E. Georgescu, Low frequency wave characteristics in the upstream and downstream regime of the terrestrial bow shock, J. Geophys. Res., 111, A01203, doi:10.1029/2005JA011231, 2006a.

Narita, Y., K.-H. Glassmeier, and R. A. Treumann, Magnetic turbulence spectra in the high-beta plasma upstream of the terrestrial bow shock, Phys. Rev. Lett., submitted, $2006 b$.

Narita, Y. and K.-H. Glassmeier, Low-frequency waves in the bow shock environment, Proc. of the Cluster and Double Star symposium - 5th anniversary of Cluster in space, (Ed) K. Fletcher, ESA SP-598, ESA Publications Division, The Netherlands, 2006.

Ness, N. F., C. S. Scearce, and J. B. Seek, Initial results of the IMP 1 magnetic field experiment, J. Geophys. Res., 69, 3531, 1964.

Ogilvie, K. W., T. von Rosenvinge, and A. C. Durney, International Sun-Earth Explorer, a three spacecraft program, Science 198, 131-138, 1977.

Omidi, N., and D. Winske, Structure of the magnetopause inferred from one-dimensional hybrid simulations, J. Geophys. Res., 100, 11,935-955, 1995.

Orlowski, D. S., C. T. Russell, and R. P. Lepping, Wave phenomena in the upstream region of Saturn, J. Geophys. Res., 97, 19,187-199, 1992. 
Orlowski, D. S., and C. T. Russell, Comparison of properties of upstream whistlers at different planets, Adv. Space Res., 16, 137-141, 1995.

Paschmann, G., N. Sckopke, S. J. Bame, J. R. Asbridge, J. T. Gosling, C. T. Russell, and E. W. Greenstadt, Association of low-frequency waves with suprathermal ions in the upstream solar wind, Geophys. Res. Lett., 6, 209-212, 1979.

Paschmann, G., N. Sckopke, I. Papamastorakis, J. R. Asbridge, S. J. Bame, and J. T. Gosling, Characteristics of reflected and diffuse ions upstream from the Earth's bow shock, J. Geophys. Res., 86, 4355-4364, 1981.

Paschmann, G., A. N. Fazakerley, and S. J. Schwartz, Moments of plasma velocity distributions, Analysis methods for multi-spacecraft data, pp. 125-158, (Eds) G. Paschmann and P. W. Daly, ISSI Scientific Report SR-001 (Electronic ed. 1.1), ISSI/ESA, 2000.

Pinçon, J. L., and F. Lefeuvre, Local characterization of homogeneous turbulence in a space plasma from simultaneous measurement of field components at several points in space, J. Geophys. Res., 96, 1789-1802, 1991.

Pokhotelov, O. A., M. A. Balikhin, R. A. Treumann, and V. P. Pavlenko, Drift mirror instability revisited: 1. cold electron temperature limit, J. Geophys. Res., 106, 84558464, 2001.

Price, C. P., D. W. Swift, and L.-C. Lee, Numerical simulation of nonoscillatory mirror waves at the Earth's magnetosheath, J. Geophys. Res., 91, 101-112, 1986.

Reiff, P. H., The use and misuse of statistical analysis, pp. 493-522, Solar-terrestrial physics - fundamental principles, ed. R. L. Carovillano and J. M. Forbes, D. Reidel, Boston, MA, 1983.

Rème, H., C. Aoustin, J. M. Bosqued, I. Dandouras, B. Lavraud, J. A. Sauvaud, A. Barthe, J. Bouyssou, Th. Camus, O. Coeur-Joly, A. Cros, J. Cuvilo, F. Ducay, Y. Garbarowitz, J. L. Medale, E. Penou, H. Perrier, D. Romefort, J. Rouzaud, C. Vallat, D. Alcaydé, C. Jacquey, C. Mazelle, C. D’Uston, E. Möbius, L. M. Kistler, K. Crocker, M. Granoff, C. Mouikis, M. Popecki, M. Vosbury, B. Klecker, D. Hovestadt, H. Kucharek, E. Kuenneth, G. Paschmann, M. Scholer, N. Sckopke, E. Seidenschwang, C. W. Carlson, D. W. Curtis, C. Ingraham, R. P. Lin, J. P. McFadden, G. K. Parks, T. Phan, V. Formisano, E. Amata, M. B. Bavassano-Cattaneo, P. Baldetti, R. Bruno, G. Chionchio, A. di Lellis, M. F. Marcucci, G. Pallocchia, A. Korth, P. W. Daly, B. Graeve, H. Rosenbauer, V. Vasyliunas, M. McCarthy, M. Wilber, L. Eliasson, R. Lundin, S. Olsen, E. G. Shelley, S. Fuselier, A. G. Ghielmetti, W. Lennartsson, C. P. Escoubet, H. Balsiger, R. Friedel, J.-B. Cao, R. A. Kovrazhkin, I. Papamastorakis, R. Pellat, J. Scudder, and B. Sonnerup, First multispacecraft ion measurements in and near the Earth's magnetosphere with the identical Cluster ion spectrometry (CIS) experiment, Ann. Geophysicae, 19, 1303-1354, 2001. 
Russell, C. T., D. D. Childers, and J. P. J. Coleman, OGO 5 observations of upstream waves in interplanetary medium: discrete wave packets, J. Geophys. Res., 76, 845-861, 1971.

Russell, C.T., Planetary bow shocks, Collisionless shocks in the heliosphere: reviews of current research, Geophysical Monograph 35, (Eds) B.T. Tsurutani and R. G. Stone, pp.109, Amer. Geophys. Union, Washington, D.C., 1985.

Russell, C. T., Multipoint measurements of upstream waves, Adv. Space Res., 8, 147-156, 1988.

Sauer, K., E. Dubinin, and J. F. McKenzie, New type of soliton in bi-ion plasmas and possible implications, Geophys, Res. Lett., 28, 3589, 2001.

Sauer, K., and E. Dubinin, Oscillitons and gyrating ions in a beam-plasma system, Geophys, Res. Lett., 30, 2192, 2003.

Scarf, F. L., D. A. Gurnett, and W. S. Kurth, Plasma wave turbulence at planetary bow shocks, Nature, 292, 747-750, 1981.

Schäfer, S., K.-H. Glassmeier, Y. Narita, K.-H. Fornaçon, M. Fränz, and I. Dandouras, Statistical phase propagation and dispersion analysis of low frequency waves in the magnetosheath, Ann. Geophysicae, 23, 3339-3349, 2005.

Scholer, M., and S. Matsukiyo, Nonstationarity of quasi-perpendicular shocks: a comparison of full particle simulations with different ion to electron mass ratio, Ann. Geophysicae, 22, 2345-2353, 2003.

Schwartz, S. J., D. Burgess, and J. J. Moses, Low-frequency waves in the Earth's magnetosheath: present status, Ann. Geophysicae, 14, 1134-1150, 1996.

Schwartz, S. J., Shock and discontinuity normals, Mach numbers, and related parameters, Analysis methods for multi-spacecraft data, (Eds) G. Paschmann and P. W. Daly, ISSI Scientific Report SR-001 (Electronic ed. 1.1), pp. 249-270, ISSI/ESA, 2000.

Sckopke, N., G. Paschmann, A. L. Brinca, C. W. Carlson, and H. Lühr, Ion thermalization in quasi-perpendicular shocks involving reflected ions, J. Geophys. Res., 95, 6337$6352,1990$.

Shue, J.-H., J. K. Chao, H. C. Fu, C. T. Russell, P. Song, K. K. Khurana, and H. J. Singer, A new functional form to study the solar wind control of the magnetopause size and shape, shocks involving reflected ions, J. Geophys. Res., 102, 9497-9511, 1997.

Smith, C. W., H. K. Wong, and M. L. Goldstein, Whistler waves associated with the Uranian bow shock - outbound observations, J. Geophys. Res., 96, 15,841-852, 1991. 
Sonett, C. P., and I. J. Abrams, The distance geomagnetic field, 3, Disorder and shocks in the magnetopause, J. Geophys. Res., 68, 1233, 1963.

Song, P., C. T. Russell, and S. P. Gary, Identification of low-frequency fluctuations in the terrestrial magnetosheath, J. Geophys. Res., 99, 6011-6025, 1994.

Sonnerup, B. U. Ö., and M. Scheible, Minimum and maximum variance analysis, Analysis methods for multi-spacecraft data, (Eds) G. Paschmann and P. W. Daly, ISSI Scientific Report SR-001 (Electronic ed. 1.1), pp. 185-220, ISSI/ESA, 2000.

Southwood, D. J., and M. G. Kivelson, Mirror instability: 1. physical mechanism of linear instability, J. Geophys. Res., 98, 9181-9187, 1993.

Sreenivasan, K. R., On the universality of the Kolmogorov constant, Phys. Fluids, 7, 2778-2784, 1995.

Stasiewicz, K., Reinterpretation of mirror modes as trains of slow magnetosonic solitons, Geophys. Res. Lett., 31, L21804, doi:10.1029/2004GL021282, 2004.

Stasiewicz, K., Theory and observations of slow-mode solitons in space plasmas, Phys. Rev. Lett., 93, 12, 2004.

Stasiewicz, K., Nonlinear Alfvén, magnetosonic, sound, and electron inertial waves in fluid formalism, J. Geophys. Res., 110, A03220, doi:10.1029/2004JA010852, 2005.

Stix, T. H., Waves in plasmas, pp. 10, Springer-Verlag, New York, 1992.

Stone, E. C., A. M. Frandsen, R. A. Mewaldt, E.R. Christian, D. Margolies, J.F. Ormes, and F. Snow, The Advanced Composition Explorer, Space Sci. Rev., 86, 1 - 22, 1998.

Thomsen, M. F., J. T. Gosling, S. J. Bame, and C. T. Russell, Gyrating ions and largeamplitude monochromatic MHD waves upstream of the Earth's bow shock, . J. Geophys. Res., 90, 267, 1985.

Tidman, D. A., and T. G. Northrop, Emission of waves by the Earth's bow shock, $J$. Geophys. Res., 73, 143, 1968.

Treumann, R. A., and W. Baumjohann, Advanced space plasma physics, pp. 341-352, Imperial College Press, London, 1997.

Treumann, R. A., and M. Scholer, The magnetosphere as a plasma laboratory, The Century of space science, 1495-1528, Kluwer Academic Publishers, The Netherlands, 2001.

Treumann, R. A., C. H. Joroschek, O. D. Constantinescu, R. Nakamura, O. A. Pokhotelov, and E. Georgescu, The strange physics of low frequency mirror mode turbulence in the high temperature plasma of the magnetosheath, Nonlinear Processes in Geophys., 11, 647-657, 2004. 
Tsurutani, B. T., E. J. Smith, R. R. Anderson, K. W. Ogilvie, J. D. Scudder, D. N. Baker, and S. J. Bame, Lion roars and nonoscillatory drift mirror waves in the magnetosheath, J. Geophys. Res., 87, 6060-6072, 1982.

Tu, C.-Y., and E. Marsch, MHD structures, waves, and turbulence in the solar wind: observations and theories, Space Sci. Rev., 73, 1-210, 1995.

Van Dyke, M., An album of fluid motion., The Parabolic Press, Stanford, CA, 1982.

Velli, M., and F. Pruneti, Alfvén waves in the solar corona and solar wind, Plasma phys. control. fusion, 39, B317-B324, 1997.

Verigin M. I., M. Tátrallyay, G. Erdős, and G. A. Kotova Magnetosheath - interplanetary medium reference frame: application for a statistical study of mirror type waves in the terrestrial plasma environment, Adv. Space Res., 35 in press, 2006.

Warhaft, Z., Turbulence in nature and in the laboratory, Proc. the National Acad. Sci., 99, 2481-2486, 2002.

Wenzel, K.-P., R. G. Marsden, D. E. Page, and E. J. Smith, The Ulysses mission, Astron. Astrophys. Suppl., 92, 207, 1992.

Wong, H. K., and M. L. Goldstein, Proton beam generation of oblique whistler waves, $J$. Geophys. Res., 93, 4110-4114, 1988.

Wu, C. S., D. Winske, Y. M. Zhou, S. T. Tsai, P. Rodriguez, M. Tanaka, K. Papadopoulos, K. Akimoto, C. S. Lin, M. M. Leroy, and C. C. Goodrich, Microinstabilities associated with a high Mach number, perpendicular bow shock, Space Sci. Rev., 37, 63-109, 1984. 



\section{Acknowledgments}

The author is most grateful to his advisor Prof. Dr. Karl-Heinz Glassmeier at the Institut für Geophysik und extraterrestrische Physik (IGEP) in Braunschweig. Having a respect to both experiments and theories and keeping balance between them, there is much to learn from the advisor. His support and respect in all situations were essential not only in pursuing this $\mathrm{PhD}$ thesis but also in growing as a scientist, never forcing any knowledge or attitudes upon the author, but rather forcing himself to keep an open mind and letting the author develop his own ideas.

The author is also very grateful to IGEP colleagues: Dr. Alexander Bogdanov who was a member of IGM (former IGEP) for granting the author of foundations about the wave analyses using magnetometer data as well as fruitful discussions and suggestions; Karl-Heinz Fornaçon, Dr. Ingo Richter, and Dr. Hans-Ulrich Auster for calibration of the Cluster FGM instruments and processing data as well as teaching the author the principles of fluxgate magnetometers. Dragoş Constantinescu for wonderful discussions on physics; Sebastian Schäfer for the implementation of the wave telescope program; IGEP ULF group (Constantinescu, Schäfer, Kleindiest, and Schmidt) for discussing wave physics and programming; Carsten Schmidt for improving the author's German disputation; Michael Rost and Kai Okrafka for assisting the author with understanding computer hardware/software; Sabine Filbrandt and Nicole Ringleb for kind assistance in coordinating travel, finding financial sources, and processing administrations.

Prof. Dr. Uwe Motschmann at the Institut für Theoretische Physik, Braunschweig, has also encouraged the author as well. Being a theoretician who pays attention to experimental physics as much as theoretical physics, his comments and questions are compact but nevertheless very accurate and illuminating in pursuing physics. The author thanks for an opportunity to share the authorship with him in the publications.

Prof. Dr. Rudolf Treumann at the Max-Planck-Institut für extraterrestrische Physik (MPE), Garching, has paid a lot of attention and interest in this $\mathrm{PhD}$ thesis. During the discussions on turbulence, he made every endeavor to bring the turbulence work into the world and visited Braunschweig all the way from Munich many times. The author looks up for his attitudes in pursuing physics as well as his talented piano play and thanks to his unselfish collaboration sincerely.

Dr. Götz Paschmann at the International Space Science Institute (ISSI), Berne, Swiss, has coordinated to summarize a lot of Cluster results in the dayside magnetospheric boundaries in to an ISSI series book. The author thanks for his kind invitation to the 


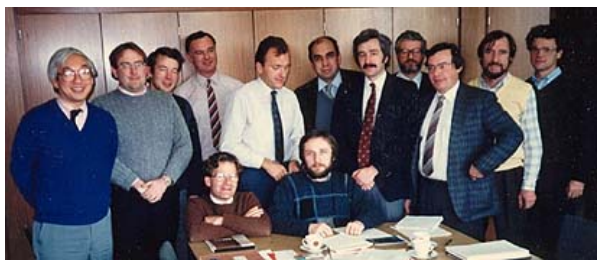

Figure 10.1: The first meeting of the scientists who proposed the magnetic field investigation on Cluster in November 1986 in TU Braunschweig. Standing, from the left, Bruce Tsurutani, Jim Slavin, Hermann Lühr, André Balogh, Günter Musmann, Mario Acuña, David Southwood, Fritz Primdahl, Konrad Schwingenschuh, Alain Meyer, Alain Roux; sitting, Rick Elphic and Karl-Heinz Glassmeier.

ISSI book and providing a chance to see a lot of famous space physicists in Berne in 2003, which was a wonderful experience.

Dr. Melvyn Goldstein at NASA Goddard Space Flight Center, USA, and Dr. Chris Gurgiolo at Bitterroot Basic Research, USA, have kindly conducted a collaboration between the Cluster FGM and PEACE (electron) investigations that led to the publication (Gurgiolo et al. 2005). The author thanks to them for their kind assistance in providing data, discussing the analysis methods and the results.

This $\mathrm{PhD}$ thesis relies significantly on the measurements made by the Cluster FGM investigation. The author is very grateful to those who have been working for nearly two decades to bring its concept into reality, especially to those who appear in Fig. 10.1. Also many thanks to Edita Georgescu at MPE for the FGM calibration, Prof. Dr. André Balogh, Dr. Elizabeth Lucek, Dr. Chris Carr, Dr. Patrick Brown, and Dr. Tim Oddy in the Imperial College, London, and all members of the Cluster FGM team.

Prof. Dr. Henri Rème, Dr. Iannis Dandouras and Dr. Elsa Chretien at Centre d'Etude Spatiale des Rayonnements (CESR), Toulouse, France assisted the author when using the ion data obtained by the CIS instruments. The author is also grateful to those who have been working with CIS for providing ion data.

The author is also grateful to Dr. Andrew N. Fazakerley and his team for providing electron data for granting a permission to use PEACE images for this thesis.

The author thanks to Dr. Philippe Escoubet at ESA for management of the Cluster mission.

Dr. Norman F. Ness and Dr. David J. McComas have kindly provided the ACE MAG and ACE SWEPAM data for this PhD work. The author is grateful to them and their respective groups.

The author is grateful to Prof. Dr. C. R. O'Dell at Vanderbilt University, NASA, and ESA for permitting to use the Hubble Space Telescope image.

The author is grateful to Dr. S. Peter Gary at the Los Alamos National Laboratory, 
Unites States, for his comments and suggestions on turbulence.

The author thanks deeply to Dr. Dieter Schmitt at the Max-Planck-Institut für Sonnensystemforschung (MPS) for his kind decision to adopt the author as a PhD student from Japan and for his organization of the International Max-Planck Research School (IMPRS).

The author is obliged to Prof. Dr. Konrad Sauer at MPS for his kind collaboration in publishing the paper Narita et al. (2003), and to Dr. Axel Korth as well as Dr. Markus Fränz for their kind assistance in the paper Narita et al. (2004).

This PhD thesis owes a lot to Dr. Masatoshi Yamauchi, Prof. Dr. Ingrid Sandahl, Prof. Dr. Rickard Lundin, Dr. Lars Eliasson, and all the colleagues at the Swedish Institute of Space Physics (IRF), Kiruna, Sweden, since an experience with the particle data of the Astrid-2 satellite in Kiruna in 1999 was very precious and inspiring for the author. Also many thanks to Dr. Olle Norberg at Esrange, Kiruna, who carried most of the Astrid-2 project on his shoulders.

The author owes also a lot to Prof. Toshifumi Mukai and Prof. Kiyoshi Maezawa at Japan Aerospace Exploration Agency, Institute of Space and Astronautical Science (JAXA/ISAS) for their complete advising in the Master degree program given at Graduate School of Tokyo University. They suggested the author to challenge the PhD degree in Braunschweig and wrote immediately a successful recommendation letter to Germany. Without their advise the author would not know how meaningful it is to study physics in Germany. The author is also grateful for Prof. Dr. Atsuhiro Nishida at Japan Society for Promotion of Science for encouraging studying physics in Germany.

The author is obliged a lot to Prof. Dr. Ryoichi Fujii at Nagoya University for coordinating the author's visit in Nagoya University and a seminar in 2004.

Remembering the days of physics courses given at Tokyo Metropolitan University in 1996-2000, the author wishes to express his best gratitude to Prof. Dr. Satoru Saito for everything he has taught, supported, encouraged. He is the one who inspired the author with the courage to always challenge physics through all his lectures and unselfishly assisted the author to challenge in Kiruna. Being not only an elementary particle physicist and a field theoretician but also a talented lecturer with very rich background experiences, he kept on giving a lively lecture and the author can not count how many first-year students at TMU were inspired by him in the right direction.

The author is also grateful to Prof. Dr. Takao Saito at Tohoku University, Japan, who immediately recommended IRF-Kiruna as a potential institute that would lead the author in the right direction in studying space physics.

Also many thanks to Takuya Masunaga and Satoshi Iijima who studied physics and mathematics at TMU together with the author in 1996-1999. Their insight in science, physics, mathematics are outstanding and they encouraged the author to keep an open mind for everything in life.

Birger Liliergren in Kiruna has been kindly exchanging letters with the author since the author stayed in Kiruna in 1999. Communicating with him in Swedish was the only hope to guess some German words for the author to survive the first half year in Braun- 
schweig.

Finally, the author thanks very much to his wife Maki, his parents Kazue and Haruhito, and big brother Norihito for supporting the author in many many situations. 


\section{Curriculum Vitae}

\section{Personal Details:}

Name: $\quad$ Yasuhito Narita

Date of Birth: November 13, 1977

Place of Birth: Nagoya, Aichi Prefecture, Japan

Nationality: Japanese

Marital Status: Married with Maki Narita

Parents: Haruhito Narita (father) and Kazue Narita (mother)

\section{Current Activity:}

Staff scientist at Institute for Geophysics and

extraterrestrial Physics, Technical University of Braunschweig since 2002

\section{Universities and Institutes:}

M.Sc. Space Physics at University of Tokyo 2002

Graduate School of Science, University of Tokyo/

Institute of Space and Astronautical Science, Sagamihara 2000-2002

B.Sc. Physics at Tokyo Metropolitan Univesity

2000

Swedish Institute of Space Physics, Kiruna, Sweden

1999

Tokyo Metropolitan University

1996-2000

\section{School Education:}

Asahigaoka Senior High School, Aichi 1993-1996

Sakurada Junior High School, Nagoya 1990-1993

Kasugano Elementary School, Nagoya 1983-1990 


\section{Publications:}

Yasuhito Narita, Plasma domain observed by ASTRID-2, IRF Scientific Report 265, ISSN 0284-1703, Kiruna, Sweden, March 2000.

H $\phi$ ymork, S. H., M. Yamauchi, Y. Ebihara, Y. Narita, O. Norberg und D. Winningham, Dense ion clouds of $0.1-2 \mathrm{keV}$ ions inside the CPS-region observed by Astrid-2, Ann. Geophysicae, 19, 621-631, 2001.

Schäfer, S., K.-H. Glassmeier, Y. Narita, K.-H. Fornaçon, I. Dandouras und M. Fränz, Statistical phase propagation and dispersion analysis of low frequency waves in the magnetosheath, Ann. Geophysicae, 23, 3339-3349, 2005. 


\section{Yasuhito Narita: Low Frequency Waves Upstream and Downstream of the Terrestrial Bow Shock}

The solar wind interacts with the Earth's magnetosphere in various ways, which causes formation of a standing shock wave called the bow shock in front of the magnetosphere. Electromagnetic waves play an important role in collisionless plasmas, since they mediate interactions between particles. The existence of low frequency waves upstream and downstream of the bow shock has been known since the discovery of the bow shock, but it was not until the advent of the Cluster mission, a four spacecraft mission, that fluctuations are clearly separated into temporal and spatial effects based on in situ observations, enabling determination of wave modes and therefore understanding wave excitation and propagation processes in those regions. The thesis presents unique wave analysis methods deriving dispersion relations, propagation patterns, and wave number spectra. The shock upstream waves are excited by the ion beam instabilities, which results from the two oppositely directed plasma streams, the solar wind and the shock reflected ions. The upstream waves exhibit properties of the fast magnetosonic waves propagating away from the shock. The downstream waves, on the other hand, represent mirror modes excited by a pressure anisotropy provided by the shock reflected and gyrating ions. They propagate inward toward the magnetopause. Turbulence properties are also discussed. 